\title{
A RISK ASSESSMENT OF A PRESSURIZED WATER REACTOR FOR CLASS 3-8 ACCIDENTS
}

Engineering and Advanced Reactor Safety Division

Department of Nuclear Energy

Brookhaven National Laboratory

Upton, New York 11973

October 1979

\section{R.E. HALL, Principle Investigator}
A. Buslik
A. Coppola (Grumman Aerospace Corp.)
M. Chun
R. Kudnik (Battelle Columbus Laboratory)
J.M. Dickey
R. Denning (Battelle Columbus Laboratory)
T. Krieger
R. Dykhuizen (Battelle Columbus Laboratory)
S. Mitra
E. Lofgren (Science Applications, Inc.)

This document is

PUBLICLY RELEASABLE

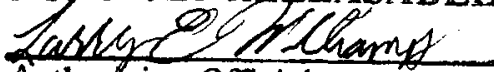

Author

Date: 04 C5Z 2007

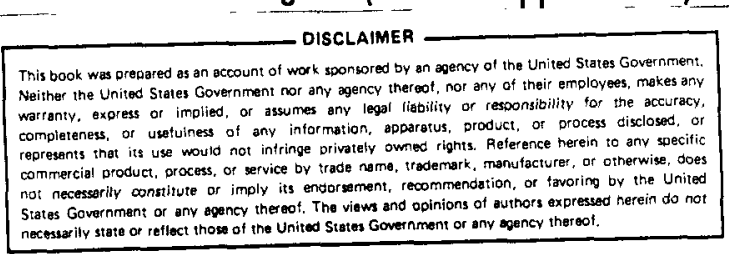

Prepared for

U.S. Nuclear Regulatory Commission

Washington, D.C. 20555

Under Interagency Agreement DE-ACO2-76CH00016

NRC FIN No. A-3057 


\section{DISCLAIMER}

This report was prepared as an account of work sponsored by an agency of the United States Government. Neither the United States Government nor any agency Thereof, nor any of their employees, makes any warranty, express or implied, or assumes any legal liability or responsibility for the accuracy, completeness, or usefulness of any information, apparatus, product, or process disclosed, or represents that its use would not infringe privately owned rights. Reference herein to any specific commercial product, process, or service by trade name, trademark, manufacturer, or otherwise does not necessarily constitute or imply its endorsement, recommendation, or favoring by the United States Government or any agency thereof. The views and opinions of authors expressed herein do not necessarily state or reflect those of the United States Government or any agency thereof. 


\section{DISCLAIMER}

Portions of this document may be illegible in electronic image products. Images are produced from the best available original document. 



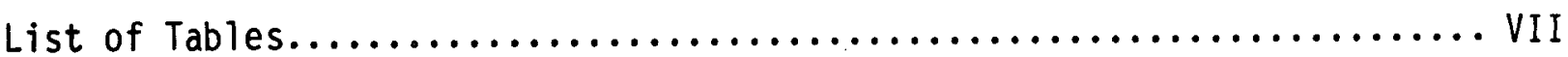

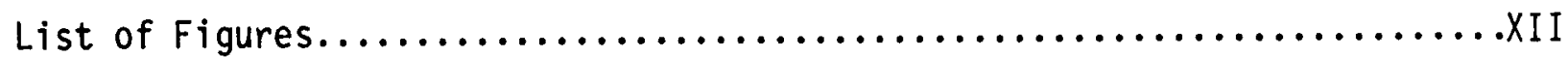

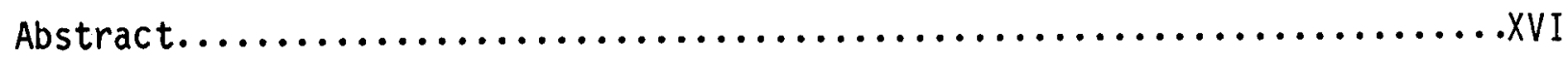

$1.0 \quad$ Introduction. $\ldots \ldots \ldots \ldots \ldots \ldots \ldots \ldots \ldots \ldots \ldots \ldots \ldots \ldots \ldots \ldots \ldots \ldots$

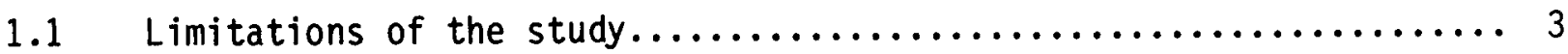

1.2 Weighting factors for radionuclides.................... 5

2.0 Analysis of data from operating experience................. 10

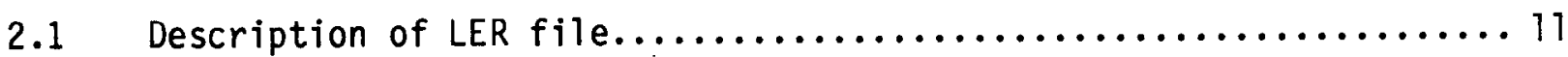

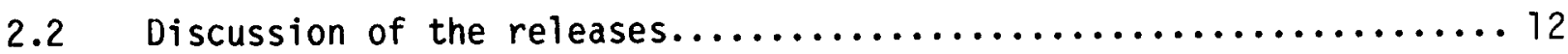

2.3 Dependence of the releases on various factors and the variation between systems............................ 14

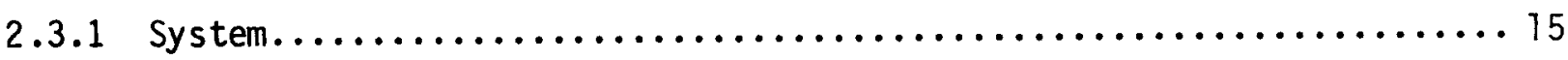

2.3 .2 Reactor Status.................................... 16

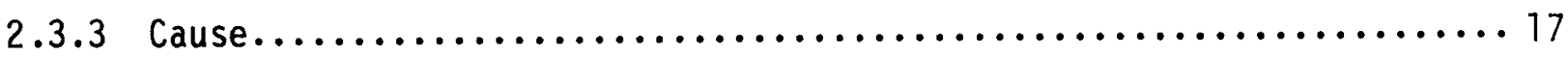

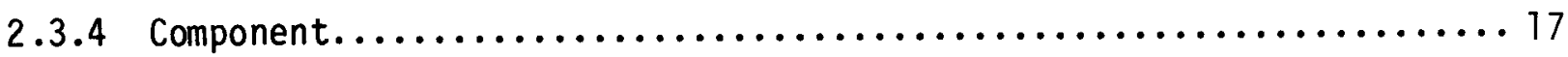

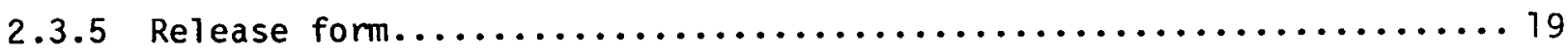

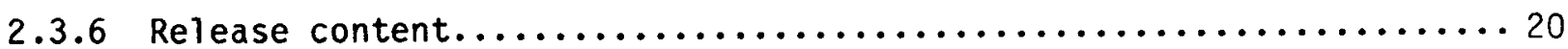

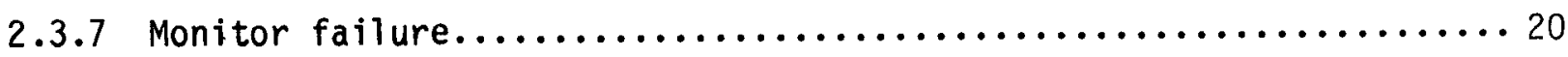

2.4 Sources of radioactivity released in the LER incidents.......... 21

2.4 .1 Theoretical radioactive inventories for surry $\ldots \ldots \ldots \ldots \ldots \ldots \ldots \ldots 21$

2.4.2 LER data on the releases related to the source

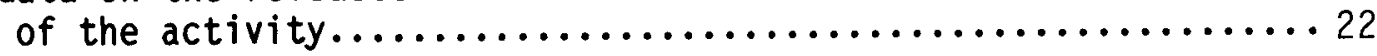

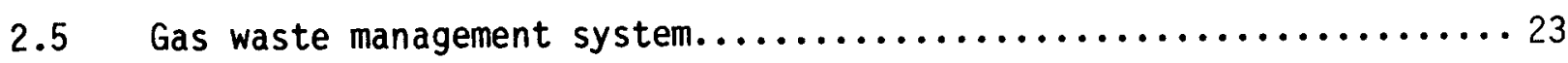

2.5 .1 Description of the gas waste management system................... 3

2.5.2 Observed releases involving the gas waste management system.......26 


\section{TABLE OF CONTENTS (Cont'd)}

Page

2.6 Results of data analysis $\ldots \ldots \ldots \ldots \ldots \ldots \ldots \ldots \ldots \ldots \ldots \ldots \ldots \ldots \ldots \ldots, 28$

3.0 Engineering analyses for Class $3-8$ accidents................ 62

3.1 Partial failure analysis................................. 64

3.2 Fission products to primary and secondary systems (Class 5$) \ldots \ldots \ldots 66$

3.2.1 Transients........................................... 66

3.2.1.1 Loss of offsite power with no compounding failures....... 69

3.2 .2 Steam generator tube rupture............................ 74

3.2.2.1 Steam generator tube rupture compounded with

loss of offsite power.............................. 78

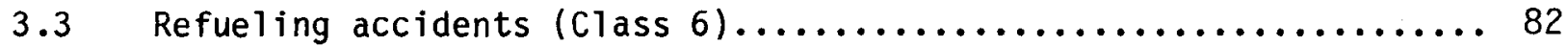

3.3.1 Inadvertent criticality during refueling $\ldots \ldots \ldots \ldots \ldots \ldots \ldots \ldots, 84$

3.3 .2 Dropping the reactor vessel head......................... 85

3.3.3 Dropping the upper core barre1......................... 85

3.3.3.1 Upper core barrel drop without isolation

or filtration.................................... 87

3.3.4 Dropping a fuel assemb1y............................... 90

3.3.4.1 Fuel assembly drop without isolation

or filtration.................................. 90

3.4 Spent fuel handling accident (Class 7$) \ldots \ldots \ldots \ldots \ldots \ldots \ldots \ldots . \ldots 1$

3.4.1 Fuel assembly drop in fuel storage pool.................... 93

3.4 .2 Heavy object drop onto fue 1 rack........................ 98

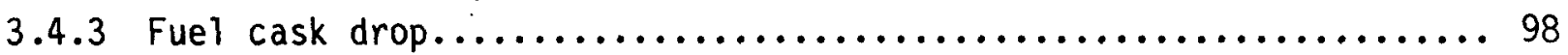

3.4.4 Loss of water/loss of cooling in spent fuel storage pool ........ 100

3.5 Accident initiating events considered in design basis evaluation in the safety analysis report (Class 8 ).............. 107

3.5 .1 Loss of coolant accident............................... 108

3.5.2 Rod ejection accident................................. 109 
3.5.3 Steamline break........................................109

3.5.3.1 Steamline break with steam generator tube rupture.

3.6 Estimation of frequency of various event sequences

in loss of feedwater (LOF) transient..........................116

3.6.1 Event trees for the loss of feedwater transient................116

3.6.2 Estimation of the probability of the events in the transient initiated by a loss of feedwater.....................117

3.6.2.1 Frequency of loss of feedwater: event (TM)............117

3.6.2.2 Probability of failure of the reactor protection system (RPOS) per demand: event (K) .................122

3.6.2.3 Unavailability of auxiliary feedwater

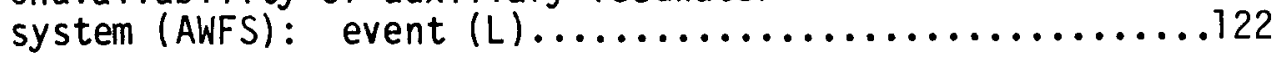

3.6.2.4 Probability of failure of a relief valve from

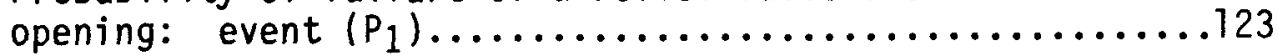

3.6.2.5 Probability of failure of safety valves from

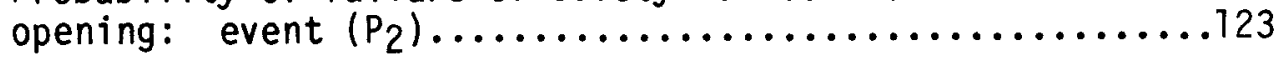

3.6.2.6 Failure probability of the relief and safety

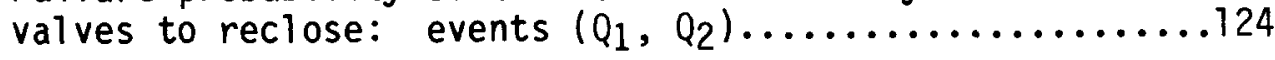

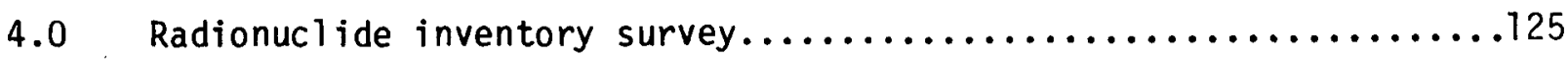

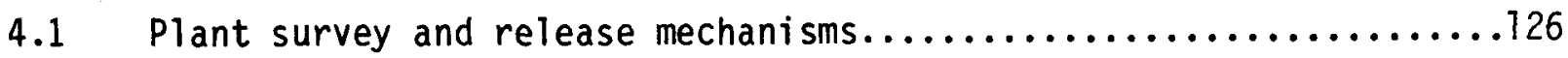

4.2 Results of survey......................................

$5.0 \quad$ Results.......................................... 130

6.0 Conclusions.......................................... 134

Appendix A - Results from Weibull curve fitting of LER data............137 Appendix B - Code description, WBFIT; a code to fit a Weibull distribution to data points........................... 150

Appendix $C$ - Waste gas decay tank inventory........................157 Appendix D - Volume control tank vapor space activity.................165 
Appendix E - Estimated activity levels in various waste disposal system tanks........................... 178

Appendix F - Models for iodine spiking and I-131 releases during a loss of offsite power shutdown................ 196

Appendix $G$ - Steam generator tube rupture with loss of offsite power................................... 221

Appendix $H$ - Core barrel drop analysis.......................... 228

Appendix I - Spent fuel handling accident in the spent fuel storage poot.................................. 231

Appendix $J$ - Spent fuel pool loss of cooling accident analysis......... 239

Appendix $K$ - Releases from the primary system initiating event of LOCA (DBA) liquid pathways........................ 285

Appendix L - Large bore pipe rupture probability as applied to a steam line break.............................. 298

Appendix M - Estimate of the likelihood of a system generator tube rupture in the event of a main steam 1 ine break....... 321

Appendix $\mathrm{N}$ - Survey of sources of radioactivity in the plant and barrier analysis........................................ 339

Acknowl edgments......................................... 373

References............................................ 374 
Figure Title

2.1 Frequency of releases reported in the LER file for each year in a plants life............................ 45

2.2a Breakdown of the releases based on the system code..................................... 45

2.2b Complementary cumulative distribution functions of the releases reported in the LER file for the gas and liquid waste management systems and the CVCS/BRS............ 46

2.2c Complementary cumulative distribution functions of the releases reported in the LER file for the primary and secondary coolant systems and the containment...............

2.3 Breakdown of the releases based on the reactor status..................................... 48

2.4a Breakdown of the releases based on the cause code........... 48

2.4b Complementary cumulative distribution functions of the releases reported in the LER file for different cause codes.............................................. 49

2.5a Breakdown of the releases based on the component code........ 50

2.5b Complementary cumulative distribution functions of the releases reported in the LER file for different components.................................. 51

2.6a Breakdown of the releases based on the form of the release.... 52

2.6b Complementary cumulative distribution functions of the releases reported in the LER file for gaseous and liquid releases.............................. 53

2.7a Breakdown of the releases based on the content of the releases................................. 54

2.7b Complementary cumulative distribution functions of the releases reported in the LER file for different contents...

2.8a Calculated CCF for Xe-133 in the gas decay tank, volume control tank and primary coolant.................... 


\section{LIST OF FIGURES (Cont'd)}

$\underline{\text { Figure }}$ Title

Page

2.8b Calculated CCF for I-131 in the gas decay tank, volume control tank and primary coolant.....................

2.8c Estimated total inventories of radionuclides

in several components.................................

2.9a Complementary cumulative distribution functions of the releases reported in the LER file in which the source of the radioactivity was the volume control tank, decay tank or the containment.

2.9b Complementary cumulative distribution functions of the releases reported in the LER file in which the source of the radioactivity was the makeup water

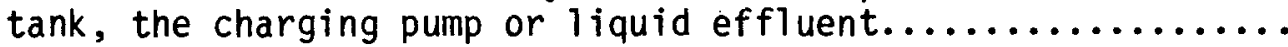

2.9c Complementary cumulative distribution functions of the releases reported in the LER file in which the source of the radioactivity was primary or secondary coolant..

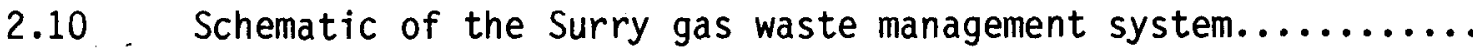

2.11 Calculated and observed CCF for releases from the

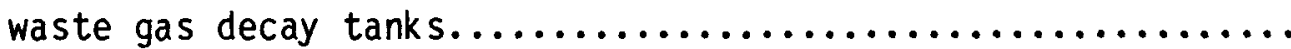

2.12 The weighted risk calculated for waste gas decay tanks compared to the LER data and the results reported in WASH-1400.

3.1 Event tree for loss of offsite power.................. 68

3.2 Probability distribution of primary to secondary leak rate... 71

3.3 CCF for time to restore offsite power............... 71

3.4 Cumulative release of steam to the atmosphere

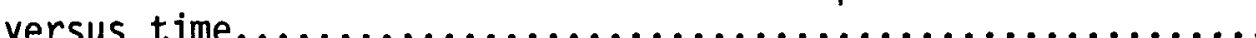

3.5 Calculated CCF for loss of offsite power accident.......... 73

3.6 The weighted risk calculated for loss of offsite power compared to the LER data and the results reported

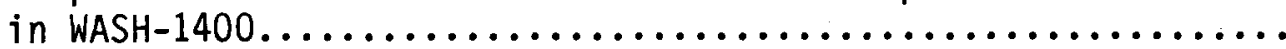

3.7 Event tree for steam generator tube rupture accident........ 
3.8 Flowrate from primary to secondary system versus time after the SGTR............................. 80

3.9 Calculated CCF for steam generator tube rupture accident.....................................

3.10 The weighted risk calculated for steam generator tube rupture compared to the LER data and the results reported in WASH-1400......................... 83

3.11 Event tree for core barrel drop........................ 86

3.12 The weighted risk calculated for core barrel drop compared to the LER data and the results

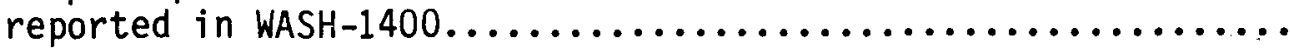

3.13 The weighted risk calculated for fuel assembly drop within contaiment compared to the LER data

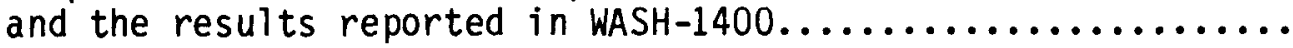

3.14 CCF for fuel assembly drop in fuel storage pool: lower and upper limits of the $50 \%$ confidence interval and the median values of the probability (per reactoryear) of exceeding $X$ curies of I-131 (inorganic) based

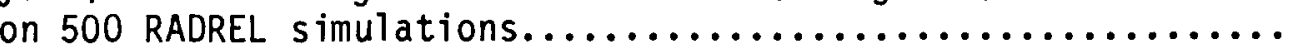

3.15 CCF for fuel assembly drop in fuel storage pool: lower and upper limits of the $50 \%$ confidence interval and the median values of the probability (per reactoryear) of exceeding $X$ curies of I-131 (organic) based

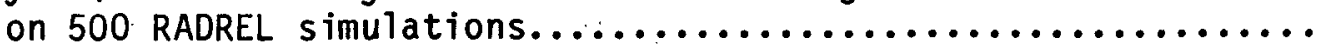

3.16 The weighted risk calculated for fuel assembly drop in spent fuel storage pool compared to the LER data

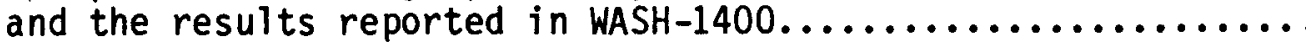

3.17 The weighted risk calculated for loss of cooling in the spent fuel pool compared to the LER data and the results reported in WASH-1400................... 100

3.18 Simplified schematic of main steam line................... 100

3.19 Event tree for steam line break accident................. 115 


\section{LIST OF FIGURES (Cont'd)}

Figure Title

Page

3.20 The weighted risk calculated for steam line break compared to the LER data and the results reported in WASH-1400.......................................... 115

3.21 Loss of feedwater event tree adapted from the generic event tree for transients given in WASH-1400......................................118

3.22 Loss of all feedwater event tree........................ 119

4.1 Schematic plot plan for Surry........................ 125

5.1 The weighted risk calculated for accident scenarios compared to the LER data and the results reported in WASH-1400. The curves for the accident scenario are: (1) loss of offsite power, (2) fuel assembly drop within containment, (3) fuel assembly drop in spent fuel storage pool, (4) steam line break, (5) core barrel drop, (6) steam generator tube rupture, (7) LOSP of cooling in spent fuel storage pool,

(8) decay tank.

5.2 Extrapolation of the weighted risk curves in the regime intermediate between the LER data and WASH-1400

A1 a\&b Estimated CCF and the fitted Weibull CCF for the releases reported in the LER file from PWR's (a) Weibull probability paper (b) linear plot for the $y$ axis...... 141

A2 a\&b Estimated CCF and the fitted Weibull CCF for the releases reported in the LER file from gas waste management system (a) Weibull probability paper (b) linear plot for the $y$ axis............................ 143

A3 a\&b Estimated CCF and the fitted Weibull CCF for the releases reported in the LER file from liquid waste management system (a) Weibull probability paper (b) linear plot for the $y$ axis.

A4 a\&b Plots on Weibull probability paper of the releases broken down according to (a) form (b) cause code In $4 \mathrm{a}$ the symbols denote o liquid, gas: In $4 \mathrm{~b}$ the symbols denote o component failure, personnel error, + procedural error. 


\section{LIST OF FIGURES (Cont'd)}

Figure Title

Page

D1 Schematic of the chemical and volume control system

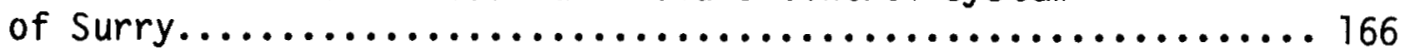

D2 Idealized schematic of stripping process in volume

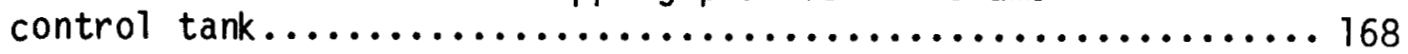

F1 Experimental data on iodine spiking compared with

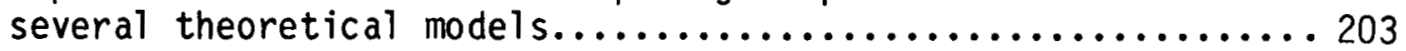

F2 Flow chart for partial failure calculation

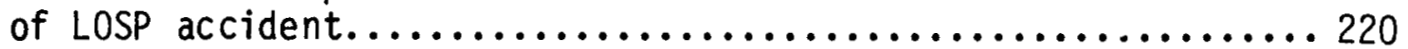

J1 Schematic fault tree for radioactivity released from the spent fuel pool............................ 254

J2 Schematic fault tree for boiling of spent fuel pool........ 258

J3 Schematic fault tree for failure of backup system.......... 259

J4 Probability distribution for repair time of pumps.......... 261

J5 Median probability of pit boiling.................. 261

J6 Error factor for probability of pit boiling...............261

J7 Dimensional schematic of Surry spent fuel pit............ 265

J8 Schematic of Surry spent fuel pit cooling system.......... 275

J9 Fault tree for boiling of spent fuel pit............. 278

K1 Estimated characteristics of Surry safety injection pumps..... 294

L1 Schematic of safety valve connection.................... 310

L2 Schematic of original nozzle and thermal sleeve.......... 314

M1 Comparison of ECT indicated and actual flaw depths for EDM slot defects.............................. 326

M2 Comparison of ECT indicated and actual flaw depths for uniform thinning defects....................... 326

M3 Comparison of ECT indicated and actual flaw depths for elliptical wastage defects..................... 326

M4 Hoop stress versus axial flaw length based on plastic instability failure analysis................... 332

$$
\text { - } \mathrm{xi} \text { - }
$$


$\underline{\text { Table }}$ Title

Page

1.1 Accident classification for environmental reports............. 2

1.2 Weighting factors for radionuclides..................... 8

2.1 Events reported in the LER file........................ 30

2.2 Releases reported in the LER file...................... 31

2.3 Major tanks in the purification and waste

processing systems.................................. 33

2.4 System involved in the release........................ 34

2.5a Breakdown of the releases for each

major system by reactor status............................ 35

2.5b Breakdown of the releases for each major

sys tem by cause code................................. 36

2.5c Breakdown of the releases for each major

system by component code............................. 37

2.5d Breakdown of the releases for each major

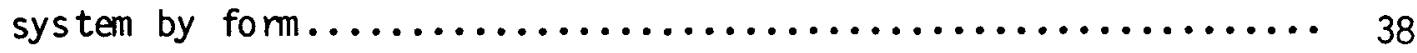

2.5e Breakdown of the releases from each major

system by content code.................................. 39

2.6 Breakdown of the releases by cause code for

each major component.................................. 40

2.7 Major sources of radioactivity releases in

the LER incidents..................................... 41

2.8 Data on the gas waste management system taken

from the Surry FSAR. ................................. 42

$2.9 \quad$ Gas waste system: releases......................... 43

2.10 Decay tank: releases................................ 44

3.1 Failed fuel probability density function................. 80

3.2 Releases for the upper core barrel drop accident........... 89 


\section{LIST OF TABLES (Cont'd)}

Table Title

3.3 Sources of variation that influence release magnitude......... 106

3.4 Sources of ignorance that influence estimation of probability versus release magnitude..................... 106

3.5 Releases for steamline break with steam generator tube rupture......................................... 115

3.6 Frequency of various event sequences in loss of

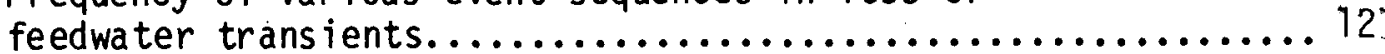

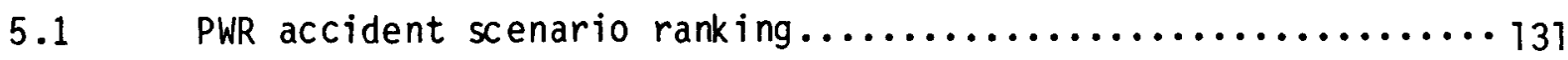

5.2 Comparison of the expected equivalent.................. 133

A1 Parameters and results of the goodness of fit tests

for the Weibull distribution.......................... 149

C1 Waste gas activity buildup............................. 161

C2 Constants of proportional ity for use in determining the probability density function for radionuclides in the waste gas decay tank............................. 162

D1 Parameters used in calculating maximum noble gas activities in volume control tank vapor space................ 179

D2 Volume control tank vapor space noble gas

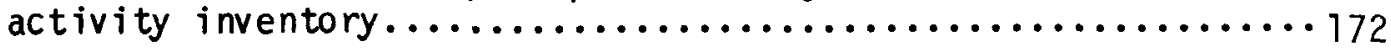

D3 Constants of proportionality for use in determining the probability density function for the radionuclide in the vapor space of the volume control tank.............. 173

D4 PWR GALE report coolant concentrations.................... 175

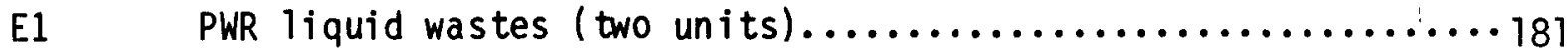

E2 PWR liquid wastes.................................. 182

E3 Calculated annual release of radioactive materials in untreated detergent waste for a PWR..................... 183

E4 Estimated liquid waste volumes (SAR data)................. 185 


\section{LIST OF TABLES (Cont'd)}

Table Title

E5 Estimated fraction of primary coolant activity for various nuclides in Boron letdown stream............... 89

E6 Estimated annual liquid waste volumes and

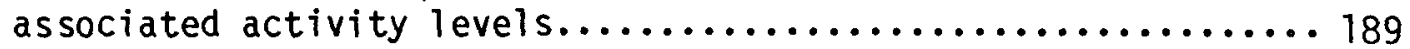

E7 Primary coolant and secondary steam activities obtained using PWR-GALE report adjustment factors........... 193

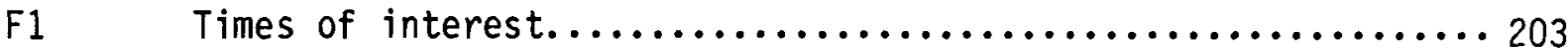

F2 Average daily leak data..................................

F3 Iodine spiking model equations, $<2$ hours................ 18

F4 Iodine spiking model equations, $>2$ hours................

I1 Calculated probability of exceeding $X$ curies of $\mathrm{Xe}-133$ released in a fuel handling accident in the

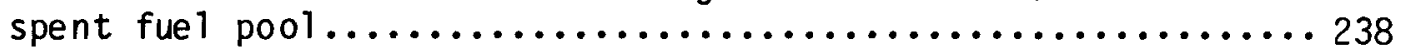

J1 Parameters used in the decay heat equations J2, J3.............................................. 241

J2 Calculated pool heat load summary........................

J3 Recommend design values for single pass methyl iodide efficiency full scale adsorbers containing impregnated activated carbon.........................250

J4 Spent fuel pit cooling system characteristics for Surry......264

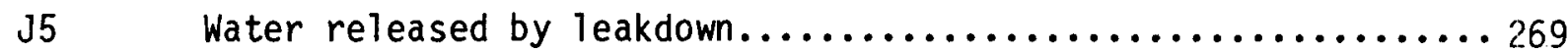

J6 Elapsed time schedules for the loss of spent fuel pit cooling water due to leakdown, siphon

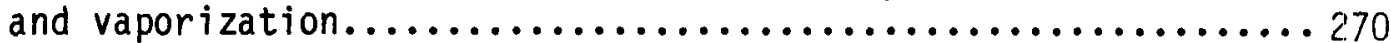

37 Summary table of time to lose cooling water from spent fuel pit......................................... 27

J8 Failure data - spent fuel pit fault tree evaluation........276

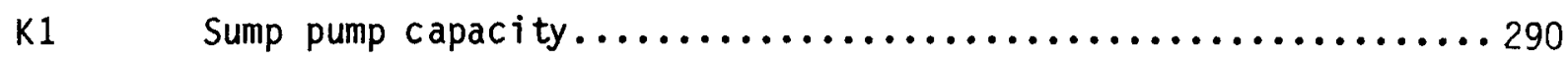


Table Title

K2 Potential waste fluid flow in a small break loss of coolant accident.............................. 294

L1 Probabilities of large bore pipe ruptures based

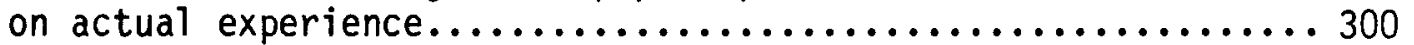

L2 Probabilities of large bore pipe ruptures based

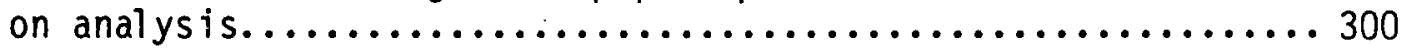

L3 Failure data and failure rate assessment of pipe failures NNPS...................................... 304

L4 Failure rate per year for three divisions of steam

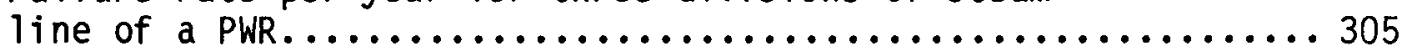

L5 Proportion of failures at various locations in

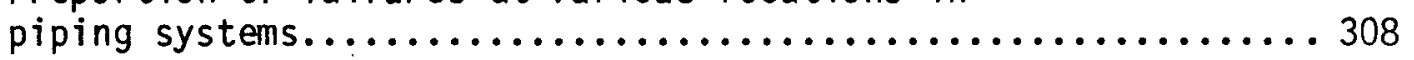

M1 Summary of interval estimates for various indicated

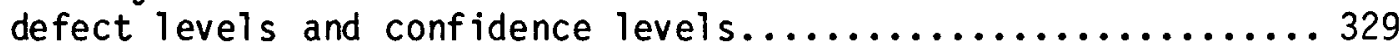

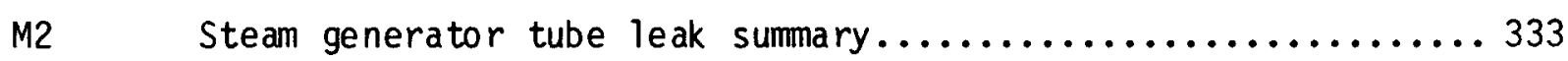

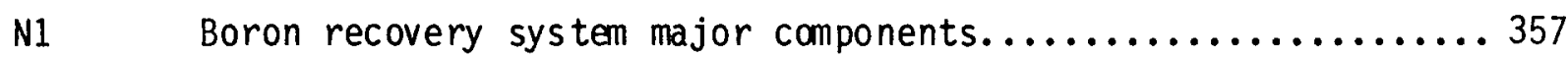




\section{ABSTRACT}

An assessment has been made of the impact on societal risk of Class 3-8 accident sequences as defined by Appendix D to 10 CFR50. The present analysis concentrates on a pressurized water reactor and utilizes realistic assumptions when practical. Conclusions are drawn as to the relative improtance of the analyzed accidents and their impact on the development of a complete societal risk curve. 


\subsection{INTRODUCTION}

This report describes the results of an investigation of the risk to the public from Class 3-8 accidents in a pressurized water reactor. Brookhaven National Laboratory (BNL) performed the investigation for the Probabilisitc Analysis Staff of the U.S. Nuclear Regulatory Commission. Science Applications Incorporated (SAI) and Battel le-Columbus Laboratories (BCL) assisted BNL in the area of probabilistic analysis and in the area of accident consequences respectively.

A classification of reactor accidents is provided in Title 10 of the Code of Federal Regulations Part 50, which is used by applicants in the preparation of Environmental Reports for nuclear power plants. Accidents in Classes 1 through 8 in this categorization fall within the review of the NRC in the licensing of plants. Class 9 accidents, which involve a number of concurrent or successive failures, could involve more severe consequences than the lower classes of accidents. Applicants are not required to analyze these accidents, however, because of their low likelihood of occurrence. The analyses performed for the Reactor Safety Study, WASH-1400(1), concentrated on Class 9 accidents involving core meltdown because they appeared to provide the greatest contribution to public risk. The purpose of the present study is to evaluate the risk from the lower classes of accidents, in a manner consistent with WASH-1400, but in greater depth. Table 1.1 identifies the Class 1-8

8 accidents which are analyzed in Environmental Reports. Classes 1 and 2 which relate to Trivial Incidents and Small Releases Outside Containment were not evaluated in this investigation because of the minor nature of the associated consequences. These classes can be considered to be included in routine releases. 
TABLE 1.1

ACCIDENT CLASSIFICATION FOR ENVIRONMENTAL REPORTS

1.0 Trivial incidents

2.0 Smali releases outside containment

3.0 Radwaste system failures

3.1 Equipment leakage or malfunction

3.2 Release of waste gas storage tank contents

3.3 Release of liquid waste storage tank contents

4.0 Fission products to primary system (BWR)

4.1 Fuel cladding defects

4.2 Off-design transients that induce fuel failures above those expected

5.0 Fission products to primary and secondary systems (PWR)

5.1 Fuel cladding defects and steam generator leaks

5.2 off-design transients that induce fuel failure above those expected and stearm generator leak

5.3 Steam generator tube rupture

6.0 Refueling accidents

6.1 Fuel bundle drop

6.2 Heavy object drop onto fuel in core

7.0 Spent fuel handling accident

7.1 Fuel assembly drop in fuel storage pool

7.2 Heavy object drop onto fuel rack

7.3 Fuel cask drop

8.0 Accident initiation events considered in design basis evaluations in the safety analysis report

8.1 Loss of coolant accidents

8.1a Break in instrument line from primary systern that penetrates the containment

8.2a Rod ejection accident (PWR)

8.2b Rod drop, accident (BWR)

8.3a Steamline breaks (PWRs outside contaiment)

8.3b. Steamline breaks (BWR) 
The approach, which was taken in estimating the risk to the public from Class 3-8 accidents, is similar to the methodology of WASH-1400. More recent data have been used in the present study. In general the evaluation of Class 3-8 accidents is able to rely more on actuarial data and less on detailed engineering analyses of accident probabilities than WASH-1400 because the frequency of the former accidents is higher. WASH-1400 used event trees with a binary, fail or success, outcome. This study extends the possible outcomes to a continuous spectrum. The methodology, which has been developed, is called "partial failure analysis." Although conservative analyses of Class 3-8 accidents have been performed for many years, methods of obtaining realistic assessments of the consequences of these accidents were not available for some accident sequences. So it has been necessary to develop special methods of analysis.

In Section 2 of this report an actuarial approach to determining tine risk of Class 3-8 accidents is described which is based on an analysis of Licensee Event Reports. In Section 3 engineering analyses are reported which extend the evaluation of risk to lower probabilities and higher consequences. A complementary study based on sources of radioactivity within the plant is described in Section 4. The Results and Conclusions are presented in Sections 5 and 6. Additional details of the analysis of specific accident sequences are in the Appendices.

\subsection{LIMITATIONS OF THE STUDY}

The analyses and conclusions provided in this report must be considered preliminary. The objective of the program, to estimate the risk of a broad 
class of accidents, is a difficult one and within the scope of this program only insights can be expected. A claim of completeness is not made for this study. Two approaches were taken to identify accident sequences. In the first approach, the accident sequences previously identified in Classes 3-8 were generalized by the use of event trees. Two workshops were held in which the project team members and NRC staff attempted to identify accident initiators or possible sequence branches for consideration. A large number of potential sequences were obtained in this review. These were reduced to a manageable number by discriminating according to probability or consequences based upon a crude assessment of each sequence. In the other approach, the various sources of radioactivity were identified within the plant and the potential paths for release to the environment were then examined. Neither approach was pursued, however, to the point where a definitive statement about completeness can be made. Indeed the completeness question for Class 3-8 acidents may be more difficult than for class 9 accidents because of the variety of ways in which a small release can occur.

In this study sequences have been evaluated in different levels of detail. A compromise was made between the desire to look at all sequences in detail and the funds available. Frequently, as a specific sequence was studied more closely, conservatisms in the analysis would be removed, and the sequence would drop in significance. Furthermore, in developing the methods of partial failure analysis, somewhat different approaches were tried for the sequences in searching for the most effective approach. Fault trees were developed for only a few sequences.

The actuarial analysis based upon Licensee Event Report data is generic in that is applied to a number of different PWR's. Most of the engineering 
analyses, on the otherhand, used the Surry power plant design and operational procedures as available to the project team. In some cases inferences about the design or operational procedures have been made which could not be checked with Virginia Electric and Power Company, (VEPCO), personnel.

A risk curve must be interpreted within the context of uncertainty bounds. In this study, the uncertainty bounds for some of the accident sequences have been examined to provide general insights as to the magnitudes of these bounds. No attempt has been made to develop uncertainty bounds for the envelope of accident risks, however.

\subsection{WEIGHTING FACTORS FOR RADIONUCLIDES}

In the analysis of Class 3-8 accidents several radionuclides, which are predicted to be released to the environment, have potential health consequences. In the regulatory review of these accidents the principal radionuclide of interest has been I-131. In order to account for other iodine isotopes a measure of "effective I-131" is frequently used which weights the other iodine isotopes in terms of their equivalent health effects. In the real istic assessment of Class 3-8 accidents, however, noble gas releases can be just as important or of greater significance than iodine. In order to be able to compare the consequences of accidents involving different mixtures of radionuclides an effort was undertaken to develop weighting factors for the radionuclides of importance. 
Before describing the approach used, we should discuss some limitations of any weighting method for radionuclide releases which is based on an attempt to duplicate the heal th effects that could result from an actual accident. In general these weighting factors will not be independent of the assumed meteorology, population distribution, accident conditions, level of release or public response to the accident. Recognizing these limitations, however, it was felt that approximate weighting factors could be obtained that would be representative of the relative contribution of radionuclides to health effects within the uncertainty bands associated with the releases.

Two groups of radionuclides were identified for which weighting factors are required. The first group consists of the xenon, krypton and iodine isotopes that would be released in Class 3-8 accidents. The second group represents the principal radionuclides released in a meltdown accident. Weighting factors for the second group are only required so that a comparison between th consequences of Class 9 accidents and Class 3-8 accidents could be made.

The resulting weighting factors are based upon results obtained with the CRAC code ${ }^{(2)}$ was developed for the WASH-1400 study. For the Class 3-8 accidents a small, $1 \mathrm{Ci}$, release of each radionuclide was made. The assumed conditions were: release at time zero, release at $2 \mathrm{~m}$ elevation, zero enthalpy of release, uniform population distribution to $500 \mathrm{miles}$, wind speed of $3 \mathrm{~m} / \mathrm{sec}$ and Pasquill D meteorology. The measure of consequence used was total latent fatalities including ten percent thyroid cancer incidence. This scaling factor for thyroid cancers is consistent with WASH-1400.(1) The health effect per curie released was then normalized to unity for I-131. A similar approach was used for the meltdown accident except that 
variations were made about a reference release. The conditions for the reference case were those of the PWR2 category of WASH-1400. The same meteorology and population distribution were used as for the Class 3-8 releases. In order to find the marginal effect of each radionuclide, the source term for the radionuclide was increased so that there was approximately a 10 percent increase in latent fatalities above the reference case. The increase in latent fatalities (including ten percent of thyroid cancer incidence) was then divided by the incremental change in the source term for that radionuclide at the time of release. The models for interdiction and decontamination introduce nonlinearities so the weight of a radionuclide at a low level of release is greater than for a large release in the CRAC analyses. Thus the marginal weights for the radionuclides obtained in the above manner will not reproduce the consequences of the reference case when multiplied by the reference inventories of all the radionuclides. In order to be able to reconstruct the consequences of the PWR2 release, the marginal weights were increased by a common factor (approximately a factor of two). These weights were then normalized to the effect of a 1 curie release of $I-131$.

The normalized weighting factors are shown in Table 1.2 for the two types of releases. Because the CRAC code does not treat organic iodine, an indirect approach was taken to infer the weighting factors for the organic iodine radionuclides from the results for noble gases. In CRAC the noble gases contribute to the irradiation of organs in the body as an external source of gamma rays. Since the calculations for the gamma source depend only on the source strength and average gamma energy, the health effects per curie of noble gas in the passing cloud are directly proportional to the photon-dose conversion factors tabulated in Table VI C-1 in WASH-1400.(1) Based on the 
T.ABLE 1.2

WEIGHTING FACTORS FOR RADIONUCLIDES

\begin{tabular}{|c|c|c|c|c|c|}
\hline Radionuclide & $\begin{array}{l}\text { Inventory } \\
\text { (Ci) } \\
\end{array}$ & $\begin{array}{l}\text { Released at } \\
\text { Failure (Ci) }\end{array}$ & Consequence & $\begin{array}{c}W F \\
(\# / C i) \\
\end{array}$ & $\begin{array}{c}\text { Normal ized } \\
\text { WF }\end{array}$ \\
\hline Kr85 & 1 & 1 & $2.89(-11)$ & $2.89(-11)$ & $2.9(-5)$ \\
\hline Kr85M & 1 & 1 & $1.49(-10)$ & $1.49(-10)$ & $1.5(-4)$ \\
\hline $\mathrm{Kr} 87$ & 1 & 1 & $3.70(-10)$ & $3.70(-10)$ & $3.7(-4)$ \\
\hline Kr88 & 1 & 1 & $1.58(-9)$ & $1.58(-9)$ & $1.6(-3)$ \\
\hline$I-131$ & 1 & 1 & $9.99(-7)$ & $9.99(-7)$ & 1.0 \\
\hline$I-133$ & 1 & 1 & $6.71(-8)$ & $6.71(-8)$ & $6.7(-2)$ \\
\hline $\mathrm{I}-135$ & 1 & 1 & $1.72(-8)$ & $1.72(-8)$ & $1.7(-2)$ \\
\hline $0 I-131$ & --- & --- & --- & --- & $3.5(-3)$ \\
\hline $0 I-133$ & -- & --- & --- & --- & $1.7(-3)$ \\
\hline $01-135$ & --- & --- & --- & $-\cdots$ & $2.3(-3)$ \\
\hline $\mathrm{Xe}-133$ & 1 & 1 & $3.27(-10)$ & $3.27(-10)$ & $3.3(-4)$ \\
\hline $\mathrm{Xe}-135$ & 1 & 1 & $3.39(-10)$ & $3.39(-10)$ & $3.4(-4)$ \\
\hline Sr90 & $2.75(7)$ & $1.43(6)$ & 58.4 & $8.03(-6)$ & 8.0 \\
\hline Ru-106 & $4.09(8)$ & $7.68(6)$ & 70.0 & $4.43(-6)$ & 4.3 \\
\hline Tel-32 & $4.8(8)$ & $1.06(8)$ & 63.5 & $2.02(-7)$ & $2.0(-1)$ \\
\hline$I-131$ & $6.02(8)$ & $3.59(8)$ & 70.5 & $9.77(-8)$ & $9.8(-2)$ \\
\hline$I-133$ & $2.21(9)$ & $1.32(9)$ & 88.9 & $5.37(-8)$ & $5.4(-2)$ \\
\hline Cs-134 & $5.2(7)$ & $2.23(7)$ & 79.2 & $2.33(-6)$ & 2.3 \\
\hline Cs-137 & $8.32(6)$ & $1.81(6)$ & 59.9 & $7.96(-6)$ & 8.0 \\
\hline $\mathrm{Ba}-140$ & $1.19(9)$ & $6.15(7)$ & 58.1 & $1.77(-7)$ & $1.8(-1)$ \\
\hline Ce-144 & $6.17(8)$ & $2.13(6)$ & 54.7 & $2.00(-6)$ & 2.0 \\
\hline
\end{tabular}

a. Meltdown releases

b. Organic iodine WF is inferred from Figure 1.

c. WF has been multiplied by 1.95 in order that the sum of weighted released yield the base case consequence of 52.5 . 
whole body conversion factors in this table, weighting factors for the organic iodines were determined. However, because the half-lives of the noble gases and iodines of interest are on the order of the transit time of the cloud through the population studied, it was necessary to account for the decay of the radionuclides. A smooth curve may be drawn through the data points given in Table 1.2, this curve aproaches a constant of proportionality for radionuclides with long half-lives. Weighting factors were obtained for organic I-131 and organic I-133 by picking the correction factor from the curve at the appropriate half-life and multiplying by the whole body dose conversion factor. It should be recognized that this procedure may underestimate weighting factors of organic iodine since some conversion to inorganic forms could occur in the environment. The results of the accident analysis did not identify a need for further refinement.

\section{Uncertainties and Variations in Weighting Factors}

An analysis was performed with other meteorological conditions to evaluate the effect on the weighting factors. The meteorological conditions for the second case were a wind velocity of $0.5 \mathrm{~m} / \mathrm{s}$ and a fifty-fifty combination of Pasquill $\mathrm{C}$ and $\mathrm{F}$ stability categories. The reference accident conditions were the same as for the PWR2 accident category. For radionuclides with half-lives greater than a day the nomalized weight factors were in good agreement with those obtained using the reference meteorology. The greatest variation for these radionuclides was less than a factor of two. Variations in the weight factors for I-133 were slightly greater than a factor of two. The weighting factors for radionuclides with short half-lives can be sensitive to the wind velocity. 


\section{ANALYSIS OF DATA FROM OPERATING EXPERIENCE}

We have currently accumulated some 200 reactor years of operating experience for commercial pressurized water reactors in the United States. Based upon this experience, it should be possible to estimate through data analysis the risk of accidental releases of radioactivity for events with a likelihood of approximately $10^{-2}$ or greater. Because the operating experiences have been obtained in reactors of different design, the measure of risk that is obtained through data analysis is generic, applying to the types of reactors included in the population studied.

of the available data sources which relate to system failures and accidents (Licensee Event Reports, Nuclear Plant Reliability Data System, NonCompliance Citations, and utility records), the most useful for examining the history of radioactivity releases is the Licensee Event Report (LER) file. (3) As will be shown, however, this system is frequently deficient in providing some of the specific detail required for these analyses.

The purpose of the effort described in this section of the report is to perform a survey of the LER file for releases from operating PWR's and through data analysis to develop an estimate of the risk of Class 3-8 accidents in the region of small consequences and high probabilities. Another objective is to examine the causes and character of radioactivity releases in order to better understand the nature of Class 3-8 accidents providing thereby a guide for the performance of engineering analyses to be used in estimating the level of risk for less likely accidents.

In addition, a significant effort was directed toward fitting the LER data to general distributions, specifically the Weibull distribution. Curve 
fitting of these data can provide a number of benefits. It can help to identify outliers or nonuniformities in the data that should be examined in greater depth. It can be used in developing uncertainty bands for the data. Finally, the expressions which are obtained can be extrapolated to extend the risk curve beyond the region in which the data were obtained. However, because of the scope of the current program limitations in this type of extrapolation was not possible. The results obtained by curve fitting the data are shown in Appendix A. Appendix B contains a description of the computer code used in the curve fitting.

\subsection{Description of the LER File}

The Licensee Event Report (LER)(3) file was established by the Atomic Energy Commission in 1973 and is currently maintained by the Nuclear Regulatory Commission. The file provides a centralized source of data on offnormal events occurring in the nuclear industry. The file consists of abstracts of more complete reports submitted by the licensee. Reporting requirements for licensed facilities are delineated in Table 10, "Energy Code of Federal Regulations" (1OCFR) and also in Technical Specifications and Licensee Provisions. The requirements may vary from facility to facility and may also be changed with time. Some pertinent factors are recorded explicitly in the entry for a report. For example, the system involved in the release, the form of release, a content code, a component code, and a cause code are all identified. It should be noted, however, that the LER file was not constructed as a quantitative source of data. The statistical inferences made from the LER file are limited by the following:

i. Not all information is available for each incident. 
ii. The quantities of radionuclides released are not reported in a uniform manner. For example, on one occasion it may be specified that $10 \mathrm{Ci}$ of I-131 is released while in another case the release might be an unspecified amount of halogens or noble gases.

iii. The accuracy of the reported releases probably varies significantly from report to report.

A search was made of the file for those reportable occurrences in which an unscheduled release of radioactivity occurred. The dates of the occurrences range from February, 1971 to November 1978; one release was in 1969. The extract from the LER file thus spans 7.8 years and 40 plants. Table 2.1 summarizes the number of events for each calendar year. The LER file is probably complete between its inception in 1973 and about October 1978. The NSIC file was used by the NRC as a source for the LER file prior to 1973. Table 2.2 shows the breakdown of the events among the plants.

The LER data file described here has been computerized to permit easy. access. Each of the factors described earlier has been tabulated together with the date and quantity of release. The data base management scheme used is System 2000.(4) This is a versatile package which allows convenient organization and access of the information. Simple mathematical operations may be performed directly. In addition, a report writer feature can be used to output selected data in $\dot{a}$ format suitable for input to Fortran programs which perform more complex calculations.

\subsection{Discussion of the Releases}

The time period covered in the extract of the LER files corresponds to 177 reactor years. For each reactor, time is measured from the date of commercial 
operation and includes down time as well as periods of operation. Two hundred sixty-six accidental releases are reported of which thirteen occurred before the state of commercial operation. Using the time span 1-1-71 to 10-1-78, the frequency of release is 1.4 per reactor year. The quantity of radioactivity released is reported in about three quarters of the occurrences. Events in which the quantity was not reported have not been considered in obtaining a normalized density function for the probability of release versus release magnitude. The overall probability of release, however, includes all events. The magnitude of the releases varies form $10^{-9} \mathrm{Ci}$ to $3085 \mathrm{Ci}$. Sixteen additional events were included in the LER output but have not been included in the present sample because they did not describe short-term accidental releases of radioactivity. Some of these events reported that the total release of effluents during a quarter exceeded technical specifications; other events reported that local samples, for example of the river, exceeded technical specifications.

The frequency of occurrence of an accidental release varies markedly in different plants as shown in Table 2.2. Besides the random statistical variation in the number of releases at a given plant, there may also be systematic trends related to the particular design of the plant. Some of the more significant factors may be the age of the plant, the size of the plant, the reactor vendor, or architect-engineer. In addition, as mentioned in 2.1 , the reporting requirements may differ from one plant to another. Because of the small size of the sample it is not possible to perform a statistical analysis of all the factors which may be pertinent. In the present study, we have selected one factor, the age of the plant, for further study. Age effects occur in both reliability engineering and human factors engineering. In both 
cases the failure rate versus age follows a 'bath tub' curve. There is an initial 'wear in' period where failure rates decrease with time, this period is called the learning phase in human factors analysis. Following this stage there is a plateau of steady failure rate and finally a 'wear out' stage where failure rates increase. The results for accidental releases of radioactivity are shown in Figure 2.1 together with estimated error bounds. The curve is terminated at 8 years because the statistics are poor for older reactors. It appears that there is a trend for the number of releases to decrease after the first two years. It should, however, be emphasized that the sampling is biased towards the smaller, older plants.

\subsection{Dependence of the Releases on Various Factors and the Variation Between Systems}

Pressurized water reactors are manufactured by three vendors with eleven architect engineering firms participating in the construction of the balance of the plant. This has resulted in considerable variation in the balance of plant design that is for these systems outside the main steam supply system. Thus the data represent an aggregation of events rather than a sample from a conventional statistical population and conclusions based on the LER data should be used with caution when applied to an individual plant. For example, an event, which has occurred at plant $A$, could not occur at plant B because of the differences of the design of plants $A$ and $B$. However, it is possible to subdivide a plant into different systems which perform similar functions in each plant although the actual design of a system may differ greatly in two different plants. For example, all plants have a system for containing and processing the gaseous radioactive wastes, but of 17 units operating prior to 1975 only 3 had a recombiner to handle the high volumes of hydrogen present in the extracted gases. 
Several factors which influence the release have been examined and will be discussed in the following sections. The first criterion for subdividing the data is based on the system primarily involved in the release. In some instances only one system is involved, in other instances several systems may be involved in the sequence of events which result in a release to the environment. For example, the radioactive material may originate in one system, the faults causing the incident may occur in a second system and the pathway for the release may be via a third system. Thus there is some ambiguity in ascribing a system to each release. The probability of a release is initially determined by the faults causing the release. So it was decided to ascribe each incident to that system in which the major fault occurred which caused the incident. In addition the actual component involved in this fault is also specified. The magnitude of a release is limited by the quantity of radioactive material at the source of the pathway to the environment. So another subdivision of the data has been made based on the source of the radioactivity. Besides the primary and secondary loops other major sources of radioactivity are tanks in the waste management and reactor coolant purification systems. Table 2.3 lists the major tanks containing radioactive liquids or gases in Surry. The detailed analysis of the releases is made difficult because in other plants the list would be different, both in the nomenclature, and size of the tanks; there may also be some tanks which are not present in Surry or conversely.

\subsubsection{System}

Each release is ascribed to the specific system primarily responsible for the release in the sense that the system is the site of the faulty condition 
faulty condition or the pathway to the environment. The choice is not always unambiguous but consistent guidelines were followed. For example, several releases occurred because of leaky tubes in the steam generator. These releases are included under the heading secondary coolant system since the pathway to the environment was via the secondary coolant system. Thus it is the characteristics of the secondary system which determine what fraction of the available radioactivity reaches the environment. Table 2.4 and Figure $2.2 \mathrm{a}$ shows the major systems involved in the releases. About 10 percent of the releases involve the primary loop in which the letdown heat exchangers and demineralizers were included. Since the concentration of fission products is high in the primary coolant these accidents could potentially lead to large releases. However, most incidents result in a release within containment. Figure 2.2b and $2.2 \mathrm{c}$ shows the complementary cumulative distribution functions (CCF) for the releases from the six major systems. The releases from the primary loop, the Chemical and Volume Control and Boron Recovery systems, (CVCS/BRS), and Gas Waste Management System tend to be larger. This is also shown in Table 2.4 where the median release is given. In the following subsections the effect of other factors on the releases is discussed and the influence of the system involved in the release on these factors is discussed.

\subsubsection{Reactor Status}

Figure 2.3 shows the status of the facility, when the release occurred. About half the releases occurred while the facility was producing power either at steady state operation or during load changes. Thirteen incidents occurred prior to the start of commercial operation. Table $2.5 \mathrm{a}$ shows the breakdown of the releases by reactor status. 


\subsubsection{Cause}

For each incident a cause code may be specified. There are five possible categories:

i. personnel error

ii. design or fabrication error

iii. external cause, such as tornado

iv. defective procedures

v. component faiture

The incidents are almost evenly divided between human error, categories I and III, and mechanical failure, categories II and V, as shown in Figure 2.4a. Only about 20 percent of the incidents led to a permanent change at the plant, either a change in procedures or a change in the design or choice of a component. The remaining incidents were due to personnel error, in that the procedures or accepted practice were not followed, or were due to a component malfunction. Table $2.5 \mathrm{~b}$ shows the breakdown of the cause code among the different systems. Human Error occurs less frequently than on the average in the Primary Coolant System and the CVCS/BRS. In these systems there are fewer routine operations which require human intervention. Figure $2.4 \mathrm{~b}$ shows the complementary cumulative distribution for the different cause codes. The shape of the distribution function does not appear to be sensitive to the cause of the release.

\subsubsection{Component}

Figure 2.5 a shows the major components involved in the releases. In 10 percent of the releases the component involved is not identified. Valves occur most frequently. The reasons for releases from valves may be 
mechanical, as in leaking seals, or can involve human error, as in the failure to close the proper valve. Table 2.6 shows the breakdown by cause code for the major components.

Several of the pipe incidents are attributed to welds leaking. Most of the heat exchanger incidents were due to tube leaks, including 16 incidents involving the steam generator. Among the components involved in only a few incidents were:

Control Rod Drive Mechanism

Demineral izers 3

Drain or Drain Trap 3

Electrical Components $\quad 5$

Filters 3

Vessel $\quad 5$

In addition, 6 incidents involved the overflow of a tank, in 8 instances no sample was taken and in 2 instances calculations were incorrect.

Table $2.5 \mathrm{c}$ shows the breakdown of the component involved for the major plant systems. Most of the releases from the secondary system involved the leakage of primary coolant through the steam generator tubes and the subsequent escape of the radioactive noble gases through the air ejector. Several of these incidents were so severe that the plant was shut down. In Figure 2.5b normalized complementary cumulative distribution functions are plotted for each basic component. No clear trends are indicated by this figure. As expected, the nature of a release is more sensitive to the systen involved and its associated inventory of radioactivity than to the various components which are present in each system. 


\subsubsection{Form of Release}

The releases are in the form of gases, liquids and solids. Figure 2.6a shows the breakdown of release by form for all the releases. Gaseous releases occur about twice as frequent as liquid releases. Table $2.5 \mathrm{~d}$ shows the form of the releases from the different systems. One release from the gas waste management system is described as a mixture, the remainder are gaseous. The water in the primary coolant system and the CVCS/BRS contains dissolved radioactive gases. In the case of a spill from these systems, the water will usually be collected in the containment or auxiliary building sumps and directed to the liquid waste management system for processing. So in most of these releases it is the gaseous products which may reach the environment. In the case of the liquid waste management system, liquid is released directly to the environment in two thirds of the releases. Figure $2.6 \mathrm{~b}$ compares nomalized complementary cumulative distributions for 1 iquid and gas releases. The gaseous releases are clearly shifted to higher releases on a curie basis. The median gaseous release is $2.4 \mathrm{Ci}$ and the median liquid release is $3.9 \times 10^{-2}$ Ci. From the viewpoint of relative heal th effects, it would be necessary to examine the content of gaseous and liquid releases as well as their pathways to man to comment on the relative importance of these two forms of release. In WASH-1400, airborne release pathways were emphasized for hypothetical core meltdown accidents because they were expected to be the most important pathways. In Class 3-8 accidents, where the hazards borne by a large population are of a statistical nature, the nature of the hazard from airborne and liquid pathways may be more similar. In the present study we have considered only the gaseous releases in detail. 


\subsubsection{Release Content}

The principal types of radionuclides released in Class 3-8 accidents are noble gases, halogens, particulates and tritium. Although there are a number of i sotopes of the noble gases released, KR-85, Xe-133 and Xe-135 are predominant. Similarly, I-131 and I-133 dominate the release of halogens. Although tritium can be released as either a gas or liquid, it is normally in the form of tritiated water. Some release of tritium would accompany virtualTy all other types of release. This breakdown is illustrated in Figure 2.7a. The majority of releases are seen to consist of noble gases. In Figure 2.7b normalized complementary cumulative distribution functions are shown for noble gas, mixture, particulate and halogen content. It should be noted that the distribution curve for the release of noble gases is seen to be shifted to higher releases by approximately $10^{4} \mathrm{Ci}$ relative to the release of halogens on a per curie basis. To a degree this shift is offset by the higher health effects of the halogen isotopes. The relative significance of halogen and noble gas releases in Class 3-8 accidents will be discussed further in Section 5. The differences between the major systems are shown in Table 2.5e.

\subsubsection{Monitor Failure}

In 17 events a radiation monitor failed. Some of the causes were electrical or mechanical, in other instances after testing the monitor was not correctly reset. The system involved in the release was the containment in 4 instances, the secondary coolant in 4 instances and the gaseous and liquid waste management systems in 8 and 1 instance respectively. 


\subsection{Sources of Radioactivity Released in the LER Incidents}

The number of curies released in an incident depends on the radioactive inventory present at the source of the release pathway, and also on the 'de-

contamination factor' for the pathway. In later sections of this report these two quantities are calculated for several specific accident scenarios. In contrast, the LER data represent the results of a statistical sample of a large number of different pathways. In order to extend the range of the results deduced from the LER data we need to consider the variation in the inventory available for release at the source of the pathway. In this section we show some theoretical results and some of the results from the data. The present discussion of sources of radioactivity released is not intended to be comprehensive, but only sufficient for the data analysis. The completeness of the study is addressed in Section 4. In the following Section 2.5, we shall apply the results to a particular example namely, the gas waste management system.

\subsubsection{Theoretical Radioactive Inventories for Surry}

The major resevoir of radioactivity in the plant is in the core. The radioactivity in the fuel rods can only be released in the event of a core melt which is outside the scope of the present study. Other major resevoirs are the spent fuel pool, the primary coolant and some of the tanks listed in Table 2.3. Appendices $D, E$, and $F$ present calculations, specific to Surry, of the radioactive inventories in the gas decay tanks, the liquid waste tanks and the volume control tanks. Figure $2.8 \mathrm{a}$ shows the complementary cumulative distribution function for the amount of Xe-133 which may be in the primary coolant, the volume control tank or the gas decay tank. The amount of Xe-133 which accumulates in the gas decay tank depends on the operating strategy as 
discussed further in Section 2.5. Figure 2.8a shows the CCF for a tank which is being continuously filled and also for a tank which has been isolated and allowed to decay for 20 days. Figure $2.8 \mathrm{~b}$ shows the CCF for I-131 for the gas decay tank and the volume control tank. The figure also shows the maximum amount of I-131 in the primary coolant and an estimated upper bound for the I-131 which could be released in the vapor form in the event of a spill of primary coolant. Figure $2.8 \mathrm{c}$ shows the total number of Curies in the liquid waste processing system tanks, and for comparison, in the core and in spent fuel.

\subsubsection{LER Data on the Releases Related to the Source of the Activity}

In contrast to the preceding section the following discussion is generic in that data from 40 plants have been combined. Since the designs of the plants vary considerably it is necessary to collect under one heading tanks which have essentially the same function. Engineering judgment must be applied in this selection. Table 2.7 lists the major sources for the releases which have occurred. Figure 2.9a, b, and $c$ show the CCF for the major sources. The larger releases originate from the primary coolant, the volume control tank and the gas decay tanks. The content of most of these releases is primarily Xe-133. Smaller releases may originate from the secondary system or as liquid effluent. The latter are included because regulations were violated during a routine release of liquid effluent. In some instances the release rate was too fast, in other releases the monitors were not functioning or a sample was not taken. 


\subsection{Gas Waste Management System}

A low level of activity is maintained in the primary coolant system because the coolant is continuously let.down and fission products are extracted. These operations are performed in the CVCS/BRS and waste management systems. In addition, any primary coolant which has leaked and all samples from the letdown systems are transferred to the waste management systems. The CVCS/BRS and the waste management systems are so complex and there are so many interconnections that there are a very large number of conceivable accident initiators which could lead to a release. So it is not practical to analyze all the possible accident scenarios as has been done in Section 3 for important scenarios involving the primary and secondary systems. An al ternative approach has been adopted. In the present chapter, the systems have been looked at from the viewpoint of the data and this study is complemented by a system survey in Section 4. We will discuss, in detail, in this section only the gas waste management system.

\subsubsection{Description of the Gas Waste Management System}

The gas waste management system processes and stores the radioactive gases produced during the operation of the reactor, both the high activity gases stripped from the reactor coolant and low activity gases arising from plant shut down, pressurizer relief tank discharge, etc.

In Surry, fission gases are stripped from the reactor coolant in the Boron recovery system and pumped to the waste gas system. In other PWR's the gases are stripped by a continuous purge of the volume control tank.

The following is a description of the Surry gas waste management system taken from the FSAR. A simplified block diagram of the waste gas system is shown in Figure 2.10. There are two waste gas decay storage tanks which are 
used for long term storage of the fission gases. This allows the activity of a11 gases other than KR-85 to decay to a fraction of the activity collected. Because the incoming gases may contain $\mathrm{H}_{2}$, provision is made to remove the $\mathrm{H}_{2}$ by passing the gases through a recombiner. Ninety-nine percent of the hydrogen is removed in the recombiner so the volume of gas is reduced to ten percent of the original volume from the gas stripper. The bleed stream from the recombiner is pumped from a small surge tank to the buried decay tanks by a diaphragm compressor.

There are two double walled gas decay tanks. The design parameters for the decay tanks and the surge tank are shown in Table 2.8. The tanks are buried for tornado protection. Sampling connections are provided for the tank contents and for leakoff in the annular intercept space between the tanks. The tanks are protected against over pressure by bellows sealed relief valves followed by rupture disc assemblies. The relief valves exit to the process vent system upstream of the filters and process vent blowers. The tanks are drained to the liquid waste processing system.

The gas waste processing system is connected to several other systems in the plant so the gas waste system may provide a pathway for the release of radioactivity from another system, or conversely radioactivity in the gas decay tanks may be released via another system. The principal connections to the gas waste system are:

- Gas stripper in the Boron Recovery System

- Volume control tank

- Containment vacuum system

- Safety injection system

- Reactor coolant drain tank 
- Pressurizer relief tank

- Boron evaporator and holdup tanks

- Liquid high level waste drain tank and evaporator

- Vent and drain system

- Steam generator blowdown

- Air ejector from condensers

- Makeup water tank

Radioactive gases from these components may be stored in the decay gas tanks or may be discharged directly through the process vent. In addition, relief valves from several systems, eg. boron recovery system, CVCS, exit to the process vent down stream of the blowers and filters. There are several plant operations which must be performed from time to time and during which a release may occur, for example venting or draining of tanks and flushing resins.

Gaseous effluents are discharged through the process vent. The decay tank contents are sampled prior to a release. The effluents are diluted with air and the dilution ratio is chosen so that the mixture is never in the hydrogen flammability regime. The discharge stream is monitored for flow rate, pressure, temperature, and particulate and gaseous activity. There are two redundant charcoal filters and two process vent blowers of $300 \mathrm{cfm}$ capacity. A slight vacuum is maintained in the charcoal filters to prevent out leakage. The process vent terminates about 10 feet above the top of the containment and the minimum exit velocity of the gas stream is about $100 \mathrm{fps}$. The monitor channel continuously withdraws a 10 scf in sample and passes the sample through a moving filter paper. The deposited activity is continuously scanned by a beta scintillation counter. The gas sample is then drawn through a sealed system to a shielded sampler enclosing a beta scintillation detector. 
The radiation monitoring system is not redundant. If the activity of the effluent stream exceeds the setting of the monitors, the release from the decay tanks is automatically terminated and containment is automatically isolated. An alarm is also in the control room.

\subsubsection{Observed Releases Involving the Gas Waste Management System}

The gas waste management system may feature in an accidental release either because it contains the source of the radioactivity released or because it serves as a pathway for the release of activity from another system. The major connections to the gas waste system are listed in the preceding section. The sources of the releases involving the gas waste system are listed in Table 2.9. It can be seen that the gas waste management system provides a pathway to the environment for a release from a large number of disparate sources. Several of the incidents were reported because technical specifications were violated during the release of effluent. For example, a sample was not taken before release or the monitors were not working. The most important source of radioactivity is the gas decay tanks.

The inventory of fission products which may be present in the decay tanks of Surry is discussed in Appendix $C$. Because the tanks may contain as much as $10^{4} \mathrm{Ci}$ of $\mathrm{Xe}-133$ we shall discuss the releases from the decay tanks in more detail and attempt to extrapolate the curves using the theoretical inventories. Table 2.10 summarizes some of the factors involved in the observed releases which originated in the decay tanks. There is a total of 19 incidents in which all or a portion of the contents of a decay tank is released. The CCF for the releases is shown in Figure $2.9 \mathrm{a}$. In order to use the data to draw theoretical curves certain assumptions must be made concerning the 
applicability of the generic data to a specific plant such as Surry. The design of the waste gas system does vary greatly from plant to plant, eg. as mentioned in Section 2.3 only 3 out of 17 older units have a recombiner. In addition, Surry is exceptional in that out of 25 units whose FSAR's were reviewed, Surry is the only unit with only 2 decay tanks; all the other units have 3 or more decay tanks. Since it has not been possible to visit Surry or to obtain first hand information about the actual operational practices used at Surry for gas waste management, conservative bounding curves were constructed for accidental releases from the gas decay tanks.

The expected inventory in the decay tank will depend on whether the tank is being continuously supplied with stripped gases, whether it is isolated, being discharged or being used during the purging of, for example, the volume control tank. The Surry FSAR describes two operating strategies; one cycle is based on alternate use of the two decay tanks with a 30 day fill, 20 day decay, 10 day bleed cycle; the other cycle assumes that the recombiner is operating and so the fill period can extend over 300 days. The inventories in the tank have been estimated in Appendix $C$ for both these cycles. In order to construct theoretical risk cures we shall conservatively assume that the decay tank which is being filled has the equilibrium inventory, this is a good approximation for most of the fill cycle. To obtain a lower bound a curve based on the inventory at the end of a 20 day decay period was constructed. The CCF of the amount of XE-133 in the tank for these two assumptions are shown in Figure 2.8a.

In order to determine the probability of a release, all the 19 incidents involving a decay tank were examined in detail. Several of these incidents occurred during some operation, such as purging the volume control tank. 
During such an operation the reserve gas decay tank is expected to be in use. Several other incidents could al so be eliminated. This left 10 incidents in which a release occurred from either the tank in service or on standby. This gives a probability of .03 per reactor year of a release from a tank which is being used to store the gases on a fill cycle. This is a very conservative estimate since it assumes that for all the incidents which occurred at several different plants a similar incident could also have taken place at Surry, despite the difference in detailed design. The volume of gas released varied in the different incidents ranging from the entire contents of the tank to 10 percent of the contents. The average release was about 0.5 of the contents of the tank. Based on these assumptions, Figure 2.11 shows the theoretical risk curve for a release of XE-133 from the gas decay tanks and for comparison the curve from the LER data. It is noticeable that the curve for surry is displaced to higher releases compared to the generic curve. This reflects the fact that Surry has only 2 decay tanks. The actual operating strategy used concerning the decay tanks will also have an effect on the curves. Since the potential releases from a gas decay tank are relatively large, further investigation of the different designs and possible operating strategies may be warranted.

\subsection{Results of Data Analysis}

The objective of the analysis of operational data has been to estimate the risk to the public in the low consequence, high probability portion of the risk spectrum. Figure 2.12 illustrates the complementary cumulative distribution functions for the LER data. Also shown on the figure for comparison is the curve obtained in WASH-1400 for core meltdown accidents. Because a curie 
of Kr85 has a different potential significance to health than a curie of I-131 or of Cs137, a better measure than curies is required for comparing the risk of different accidents. In Figure 2.12 weighting factors from Table 1.2 were applied to the different releases to obtain a more meaningful comparison. Also shown are the two bounding curves for the estimated risk due to a release from a waste gas decay tank. In addition to the development of the high probability portion of the risk spectrum, data analysis has been used to identify important release forms, radionuclides, accident causes and systems for consideration in the engineering analyses described in the following section. 
TABLE 2.1

\section{EVENTS REPORTED IN THE LER FILE}

\begin{tabular}{cccc} 
Year & $\begin{array}{c}\text { Number of } \\
\text { Events }\end{array}$ & $\begin{array}{c}\text { Number of } \\
\text { PWR's On Line }\end{array}$ & Reactor Years \\
\cline { 2 - 3 } 1978 & 28 & 40 & 39.1 \\
1977 & 46 & 38 & 35.0 \\
1976 & 38 & 32 & 30.1 \\
1975 & 67 & 29 & 26.7 \\
$1974^{\star}$ & 58 & 26 & 19.5 \\
1973 & 14 & 18 & 13.2 \\
1972 & 6 & 11 & 8.3 \\
1971 & 9 & 8 & 6.8 \\
1970 & 0 & 6 & 4.5 \\
1969 & 1 & 4 & 4.0 \\
1978 & 267 & 41 & 203.0
\end{tabular}

*Indian Point 1 was shut down on October 31, 1974. 
TABLE 2.2

RELEASES REPORTED IN THE LER FILE

\begin{tabular}{|c|c|c|c|c|c|}
\hline Plant Name & $\begin{array}{c}\text { Capacity } \\
\text { MWE }\end{array}$ & $\begin{array}{r}\text { Commercial } \\
\text { Operation } \\
\end{array}$ & $\begin{array}{c}\text { Age of plant } \\
\text { on } 10 / 1 / 78 \\
\end{array}$ & $\begin{array}{r}\text { No. of } \\
\text { Releases } \\
\end{array}$ & Maximum (Ci) \\
\hline Yankee & 175 & $07 / 01 / 61$ & 17.263 & 14 & 130.000000000 \\
\hline Indian Point 1 & 265 & $10 / 01 / 62$ & 16.011 & 8 & 5.700000000 \\
\hline San Onofre & 436 & $01 / 01 / 68$ & 10.756 & 1 & 0.000002700 \\
\hline Haddam Neck & 575 & $01 / 01 / 68$ & 10.756 & 16 & 266.870000000 \\
\hline Robert E. Ginna & 490 & $07 / 01 / 70$ & 8.258 & 10 & 228.000000000 \\
\hline Point Beach 1 & 497 & $12 / 21 / 70$ & 7.784 & 20 & 3085.000000000 \\
\hline H. B. Robinson & 665 & $03 / 07 / 71$ & 7.575 & 8 & 0.379290000 \\
\hline Palisades & 700 & $12 / 31 / 71$ & 6.756 & 16 & 16.400000000 \\
\hline Point Beach 2 & 497 & $10 / 01 / 72$ & 6.003 & 2 & 0.006060000 \\
\hline Surry 1 & 775 & $12 / 22 / 72$ & 5.778 & 19 & 319.700000000 \\
\hline Maine Yankee & 790 & $12 / 28 / 72$ & 5.762 & 3 & 0.810000000 \\
\hline Surry 2 & 775 & $05 / 01 / 73$ & 5.422 & 2 & $0.35000000 \theta$ \\
\hline Oconee 1 & 871 & $07 / 15 / 73$ & 5.216 & 13 & 157.000000000 \\
\hline Indian Point 2 & 873 & $08 / 01 / 73$ & 5.170 & 8 & 60.400000000 \\
\hline Turkey 4 & 666 & $09 / 07 / 73$ & 5.068 & 5 & 2.100000000 \\
\hline Fort Calhoun 1 & 457 & $09 / 26 / 73$ & 5.016 & 18 & 1046.000000000 \\
\hline Prairie Island 1 & 520 & $12 / 16 / 73$ & 4.795 & 3 & 0.770000000 \\
\hline Zion 1 & 1100 & $12 / 31 / 73$ & 4.753 & 13 & 172.000000000 \\
\hline Kewaunee & 535 & $06 / 01 / 74$ & 4.337 & 5 & \\
\hline Three Mile Island 1 & 792 & $09 / 02 / 74$ & 4.082 & 25 & 60.000000000 \\
\hline Oconee 2 & 871 & $09 / 09 / 74$ & 4.063 & 6 & 104.000000000 \\
\hline
\end{tabular}


TABLE 2.2 (Cont'd)

RELEASES REPORTED IN THE LER FILE

\begin{tabular}{|c|c|c|c|c|}
\hline Plant Name & $\begin{array}{c}\text { Capacity } \\
\text { MWE }\end{array}$ & $\begin{array}{l}\text { Commercial } \\
\text { Operation } \\
\end{array}$ & $\begin{array}{l}\text { Age of plant } \\
\text { on } 10 / 1 / 78 \\
\end{array}$ & $\begin{array}{r}\text { No. of } \\
\text { Release }\end{array}$ \\
\hline Zion 2 & 1100 & $09 / 17 / 74$ & 4.041 & \\
\hline Turkey 3 & 666 & $12 / 14 / 74$ & 3.800 & \\
\hline Oconee 3 & 871 & $12 / 16 / 74$ & 3.795 & \\
\hline Arkansas 1 & 836 & $12 / 19 / 74$ & 3.786 & \\
\hline Prairie Island 2 & 520 & $12 / 21 / 74$ & 3.781 & \\
\hline Rancho Seco & 913 & $04 / 17 / 75$ & 3.460 & \\
\hline Calvert $\mathrm{Cl}$ iffs 1 & 850 & $05 / 08 / 75$ & 3.403 & \\
\hline Donald C. Cook 1 & 1054 & $08 / 27 / 75$ & 3.099 & \\
\hline Millstone 2 & 828 & $12 / 26 / 75$ & 2.767 & \\
\hline Trojan & 1130 & $05 / 20 / 76$ & 2.367 & \\
\hline Indian Point 3 & 965 & $08 / 30 / 76$ & 2.088 & 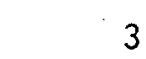 \\
\hline St. Lucie 1 & 802 & $12 / 21 / 76$ & 1.778 & 2 \\
\hline Crystal River 3 & 825 & $03 / 13 / 77$ & 1.553 & 16 \\
\hline Calvert Cliffs 2 & 850 & $04 / 01 / 77$ & 1.501 & 0 \\
\hline Beaver Valley 1 & 852 & $04 / 01 / 77$ & 1.501 & 1 \\
\hline Salem & 1090 & $06 / 30 / 77$ & 1.255 & 1 \\
\hline Davis-Besse 1 & 906 & $11 / 20 / 77$ & .863 & 0 \\
\hline J. Farley 1 & 860 & $12 / 01 / 77$ & .833 & 4 \\
\hline N. Anna 1 & 934 & $06 / 06 / 78$ & .321 & 1 \\
\hline Donald C. Cook 2 & 1100 & 07/01/78 & .252 & 0 \\
\hline
\end{tabular}

\section{Maximum (Ci)}

31.800000000

0.079000000

0.262000000

8.990000000

46.000000000

4. 320000000

0.100000000

62.500000000

12.680000000

114.000000000

185.000000000

3.400000000

0.000001397

0.262000000

0.000525000 
MAJOR TANKS IN THE PURIFICATION

AND NASTE PROCESSING SYSTEMS*

System

Gas Waste Management

Liquid Waste

Management

Vent and Drain

Chemical and Volume Control

Boron Recovery

Primary drain

Gas stripper

Gas stripper surge

Boron recovery

Boron evaporator

Distillate accumulator

Test tank

Primary grade water storage

Boron evap. bottoms
Number Vol (gal)

$\begin{array}{cc}2 & 434\left(\mathrm{ft}^{3}\right) \\ 1 & 15.7^{11}\end{array}$

$2 \quad 2,390$

$1 \quad 290$

2548

$2 \quad 2,874$

$2 \cdot 1,230$

1250

12,019

$1 \quad 619$

$\begin{array}{lr}2 & 600 \\ 2 & 20 \\ 2 & 5 \\ & \\ 1 & 300\left(\mathrm{ft}^{3}\right) \\ 1 & 5 \\ 1 & 800 \\ 3 & 7,500 \\ 1 & 635\end{array}$

$\begin{array}{lr}1 & 5,000 \\ 1 & 1,248 \\ 1 & 525 \\ 3 & 120,000 \\ 2 & 2,900 \\ 2 & 550 \\ 2 & 30,000 \\ 2 & 180,000 \\ 1 & 4,000\end{array}$

*Taken from the Surry FSAR. 
TABLE 2.4

\section{SYSTEM INVOLVED IN THE RELEASE}

System

Gas Waste Management

Liquid Waste Management

Chemical Volume Control/

Boron Recovery

Primary Loop

Secondary Loop

Contajnment

Spent Fuel Pool

Demineral ized Water

Sampling

Component Cooling Water

Miscellaneous or Unspecified

A11 Systems
Number of Releases

61

53

42

30

25

18

9

7

5

4

13

266
Median Release (Ci)

3

0.1

1

4

0.5

0.6

.03

.2

.9

1

.84 
TABLE 2.5a

BREAKDOWN OF THE PERCENTAGE OF RELEASE

FROM EACH MAJOR SYSTEM BY REACTOR STATUS

LC PRE : R $\quad S \quad$ SDN $\quad$ SS $\quad$ SUP Unspecified Total

\begin{tabular}{|c|c|c|c|c|c|c|c|c|c|}
\hline CVCS/BRS & 2.4 & 2.4 & 2.4 & 16.7 & 2.4 & 61.9 & 7.1 & 4.7 & 42 \\
\hline $\begin{array}{l}\text { Gas Waste } \\
\text { Management }\end{array}$ & 4.9 & 1.6 & 1.6 & 14.7 & 4.9 & 60.6 & 0 & 11.7 & 61 \\
\hline $\begin{array}{l}\text { Liq. Waste } \\
\text { Management }\end{array}$ & 7.5 & 7.5 & 5.7 & 11.3 & 0 & 41.5 & 7.5 & 19 & 53 \\
\hline $\begin{array}{l}\text { Primary } \\
\text { Coolant }\end{array}$ & 3.3 & 6.7 & 10 & 20 & 0 & 36.7 & 6.7 & 16.6 & 30 \\
\hline $\begin{array}{l}\text { Secondary } \\
\text { Coolant }\end{array}$ & 4 & 0 & 8 & 24 & 0 & 56 & 4 & 4 & 25 \\
\hline Containment & 0 & 0 & 11.1 & 11.1 & 5.5 & 61.2 & 0 & 11.1 & 18 \\
\hline All systems & 3.7 & 3 & 7.8 & 16.5 & 2.2 & 49.2 & 4.5 & 13.1 & \\
\hline
\end{tabular}


TABLE 2.5b

BREAKDOWN OF THE RELEASES FOR

EACH MAJOR SYSTEM BY CAUSE CODE

System

Cause Code

CVCS

Gas Waste

Management

Liq. Waste Management

Primary Coolant

Secondary

Coolant

Containment

All Systems
\% Component \%Personnel \% Defective

Failure

Error Procedure

\% Design Total No.

61.9

7.2

7.1

23.8

42

47.5

31.1

11.5

4.9

61

32

41.5

17

4

53

46.7

36.7

13.3

3.4

30

76

12

12

25

33.3

44.5

16.7

18

$\begin{array}{llll}47.7 & 29.5 & 12.7 & 6.7\end{array}$


TABLE $2.5 \mathrm{C}$

BREAKDOWN OF THE RELEASES FROM EACH MANOR SYSTEM BY COMPONENT CODE

System \% Heat

Component Code Exchanger \% Instrumentation \% Pipe \& vaive Total No.

CVCS

$30.9 \quad 45.2 \quad 42$

Gas Waste

Management

6.6

9.8

19.7

29.5

61

Liq. Waste Management

3.8

11.3

11.3

26.4

53

Primary

Coolant

3.3

3.3

13.3

36.7

30

Secondary

Coolant

64

12

4

8

25

Containment

22.2

44.5

18

All Systems

10.4

8.2

14.2

31 
TABLE 2.5d

\section{BREAKDOWN OF THE RELEASE FOR EACH MAJOR SYSTEM BY FORM}

\begin{tabular}{|c|c|c|c|c|c|}
\hline System Form & $\%$ Gas & $\%$ Liquid & \% Mixture & $\%$ Solid & Total No. \\
\hline CVCS & 69 & 16.7 & 12 & 2.3 & 42 \\
\hline $\begin{array}{l}\text { Gas Waste } \\
\text { Management }\end{array}$ & 98.4 & 0 & 1.6 & 0 & 61 \\
\hline
\end{tabular}

Liq. Waste

Management

$\begin{array}{lllll}26.4 & 69.8 & 3.8 & 0 & 53\end{array}$

Primary

Coolant

86.7

10

3.3

0

Secondary

Coolant

48

40

12

0

All Systems

65.0

29.5

4.8

0.7

30

25

53 
TABLE 2.5e

BREAKDOWN OF THE RELEASES FROM

EACH MAJOR SYSTEM BY CONTENT CODE

System

Content Code

\% Halogen \% Mixture \% Noble \% Particulate Total No.

CVCS

4.8

23.8

59.5

2.4

42

Gas Waste

Management

8.

16.4

68.8

1.6

61

Liq. Waste

Management

$3.8 \quad 39.6$

20.7

13.2

53

Primary

Coolant

10

20

53.3

6.7

30

Secondary

Coolant

Conta i nment

5.6

16.7

4.4

16.7

18

A11 Systems

4.8

24.6

39.5

5.2 
TABLE 2.6

BREAKDOWN OF THE RELEASES BY

CAUSE CODE FOR EACH MAJOR COMPONENT

\begin{tabular}{|c|c|c|c|c|c|}
\hline $\begin{array}{l}\text { Cause Code } \\
\text { Component }\end{array}$ & \% Design & $\begin{array}{c}\% \text { Component } \\
\text { Failure } \\
\end{array}$ & $\begin{array}{c}\% \text { Personnel } \\
\text { Error } \\
\end{array}$ & $\begin{array}{c}\% \text { Procedural } \\
\text { Error } \\
\end{array}$ & Total No. \\
\hline Valve & 3.6 & 52 & 31 & 13 & 83 \\
\hline Pipe & 18.5 & 50 & 21 & 11 & 38 \\
\hline $\begin{array}{l}\text { Heat } \\
\text { Exchanger }\end{array}$ & 3.5 & 93 & 0 & 3.5 & 28 \\
\hline Instrumentation & 0 & 50 & 41 & 4.5 & 22 \\
\hline Pump & 11.8 & 65 & 6 & 18 & 17 \\
\hline Al1 Components & 7 & 53 & 27 & 11 & \\
\hline
\end{tabular}


TABLE 2.7

MAJOR SOURCES OF RADIOACTIVITY

RELEASED IN THE LER INCIDENTS

Primary Coolant

Liquid Effluent 22

Charging Pump 21

Decay Tank 20

$\begin{array}{ll}\text { Containment } & 18\end{array}$

Makeup Water Tank $\quad 15$

Volume Control Tank 13

Secondary Coolant $\quad 13$

Boron Recovery Tank 11

High Level Waste Tank 8

Refueling Water Storage Tank 5

Primary Drain Tank $\quad 5$

Low Level Waste Tank 3 
TABLE 2.8

DATA ON THE GAS WASTE MANAGEMENT SYSTEM

TAKEN FROM THE SURRY FSAR

\section{Waste Gas Decay Tanks}

Number

2

Capacity Each, $\mathrm{ft}^{3}$

434

Design Pressure

Outer Tank

Inner Tank

From $30 \mathrm{in}$. $\mathrm{Hg}$ vacuum to $150 \mathrm{psig}$

From 30 in. $\mathrm{Hg}$ vacuum to $150 \mathrm{psig}$

Design Temperature, ${ }^{\circ} \mathrm{F}$

200

200

Operating Pressure, psig

Atmospheric

115

Operating Temperature, ${ }^{\circ} \mathrm{F}$

120

140

Material

Carbon

Stainless Steel

Type 304L

Design Code

ASME VIII

ASME III C

Earthquake Design

Complies with Class I Requirements

Waste Gas Surge Tank

Number

1

Capacity, $\mathrm{ft}^{3}$

15.7

Design Pressure

From $30 \mathrm{in}$. $\mathrm{Hg}$ vacuum to $30 \mathrm{psig}$

Design Temperature, ${ }^{\circ} \mathrm{F}$

300

Operating Pressure, psig

15

Operating Temperature, ${ }^{\circ} \mathrm{F}$

120

Material

Stainless Steel Type 304

Design Code

ASME III C 
TABLE 2.9

GAS WASTE SYSTEM: RELEASES

Internal Source

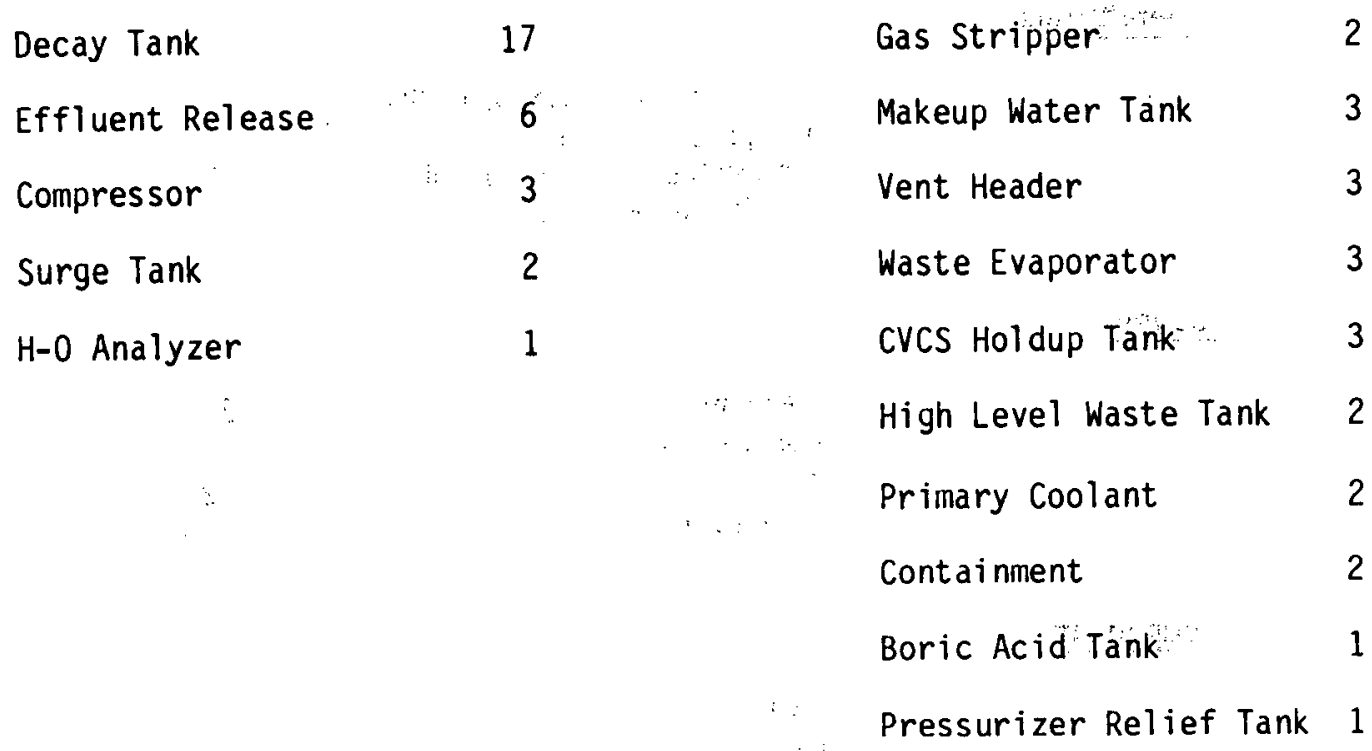

\section{External Source}

2

3

3

3

3

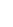
2 1 
TABLE 2.10

DECAY TANK: RELEASES

\title{
Reactor Status
}

\author{
Steady State \\ Load Changes \\ Shutdown \\ Refueling
}

Operations

Vent or Fill Vessel (R)

Purge or Drain VCT

Cover Gas to Holdup Tank

Drain Decay Tank

Cause

$$
\begin{aligned}
& \text { Failure } \\
& \text { Personnel } \\
& \text { Design } \\
& \text { Procedural }
\end{aligned}
$$

Component

Valve

Pipe

Instrumental

Drain, Drain Trap

Heat Exchanger

Releases of Stored Tank

Rupture Disc Failed

Valve Failed Open

Over Pressurization

Wrong Tank Released

3
2
2
1 


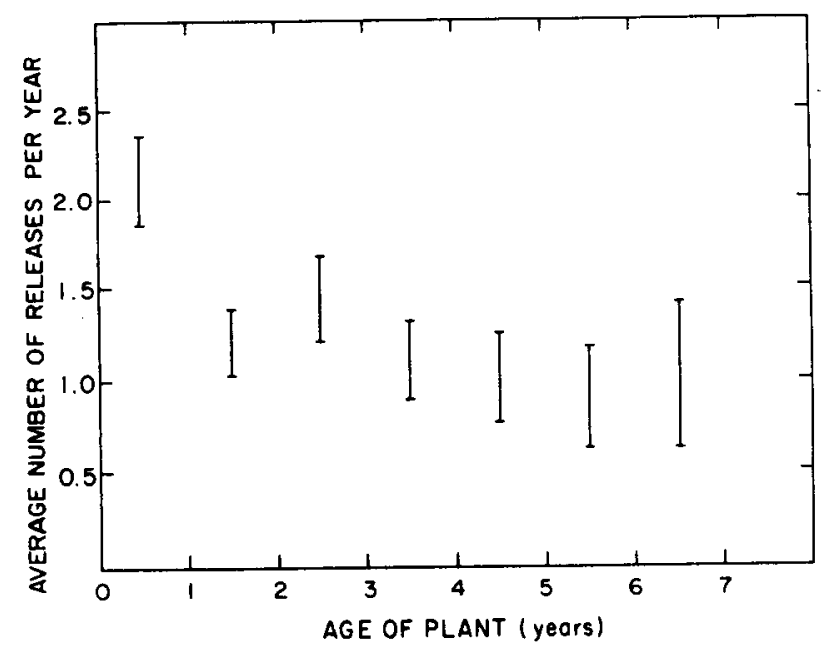

Figure 2.1. Frequency of releases reported in the LER file for each year in a plants life

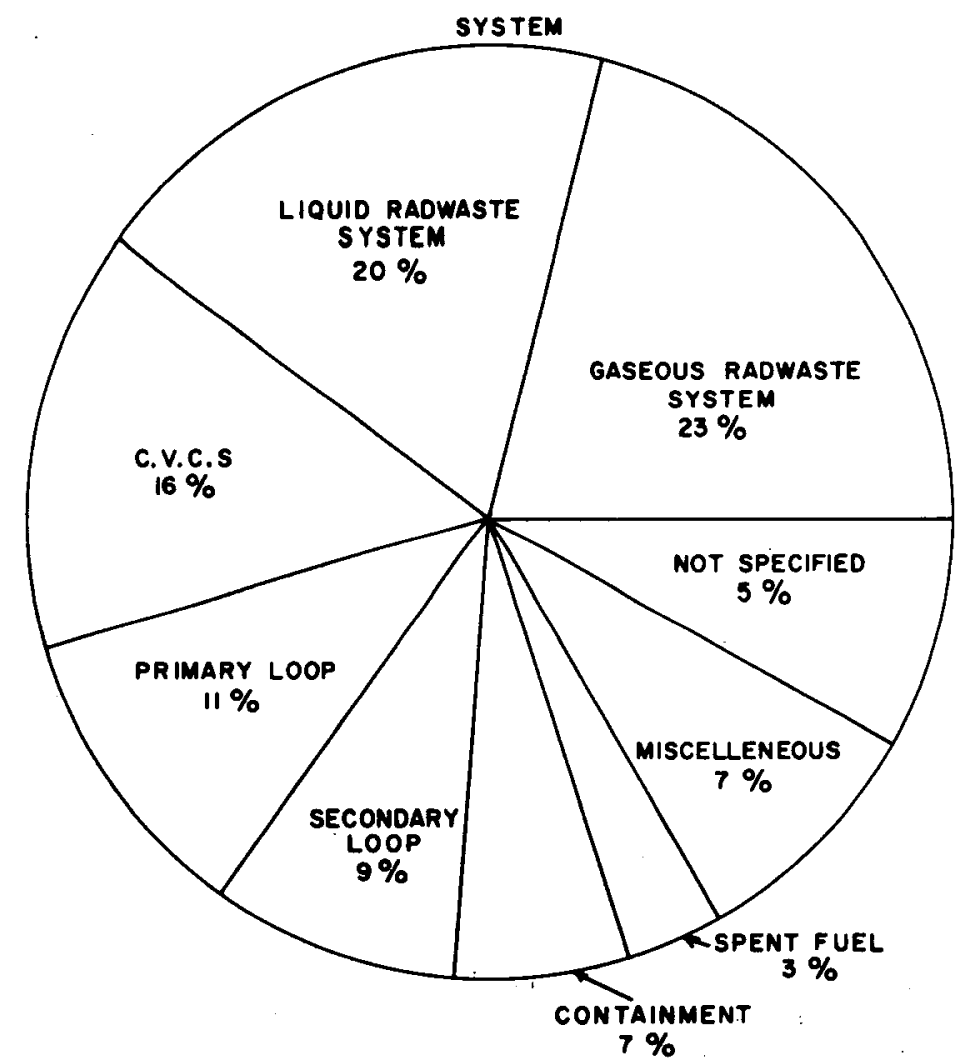

Figure 2.2a. Breakdown of the releases based on the system code 


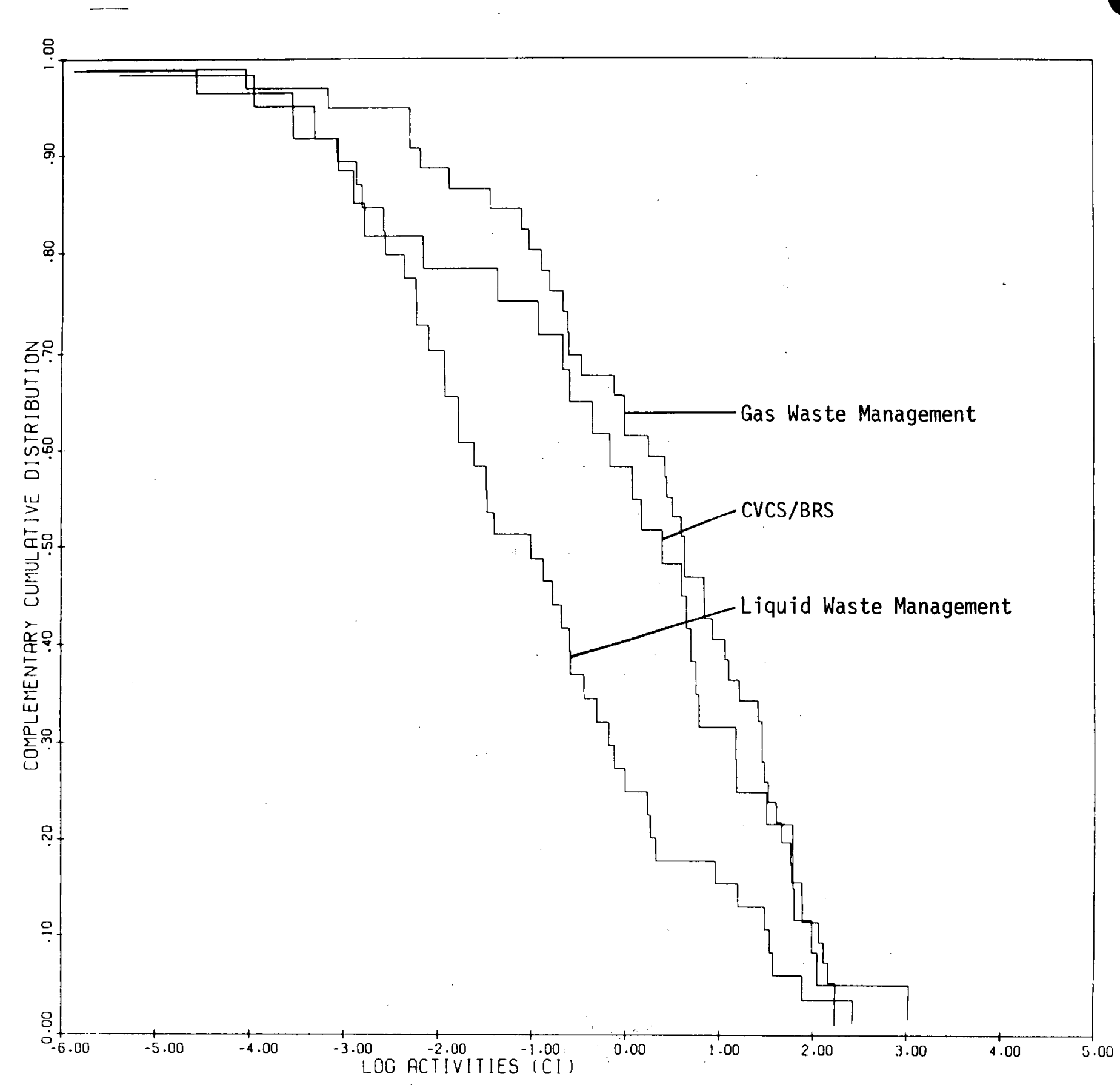

Figure 2,2b. Complementary cumulative distribution functions of the releases reported in the LER file for the gas and liquid waste management systems and the CVCS/BRS. 


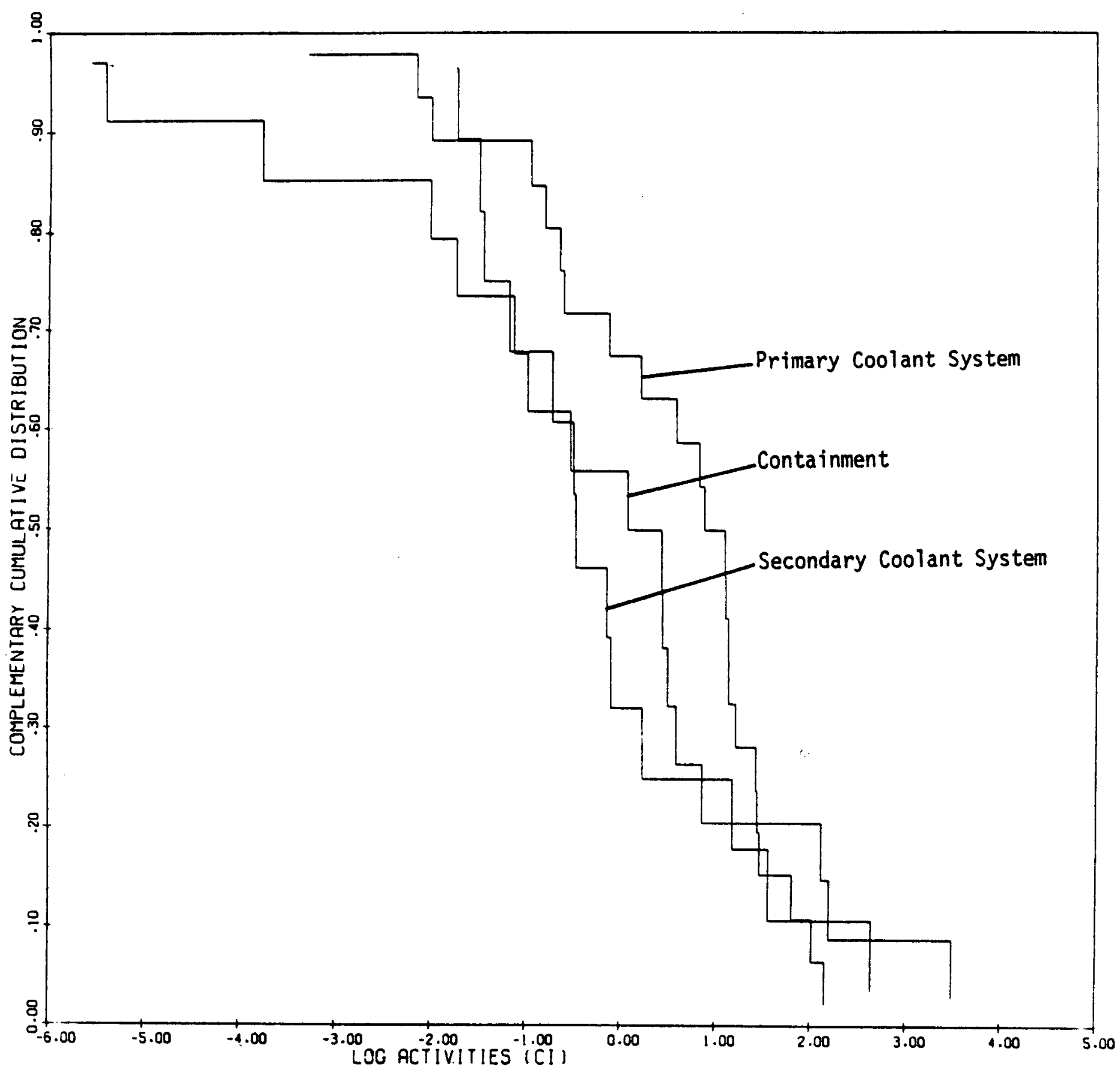

Figure 2.2c. Complementary cumulative distribution functions of the releases reported in the LER file for the primary and secondary coolant systems and the containment. 


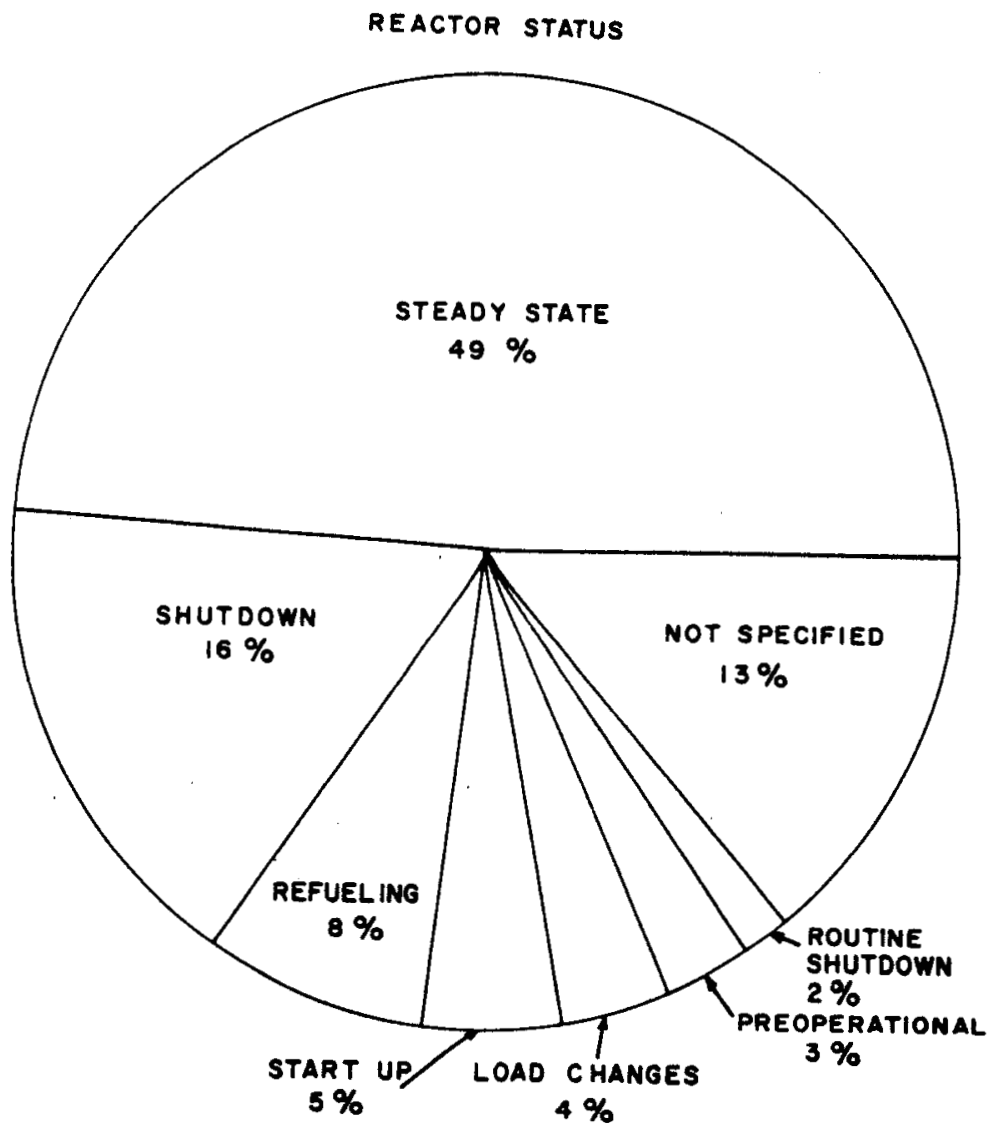

Figure 2.3. Breakdown of the releases based on the reactor status.

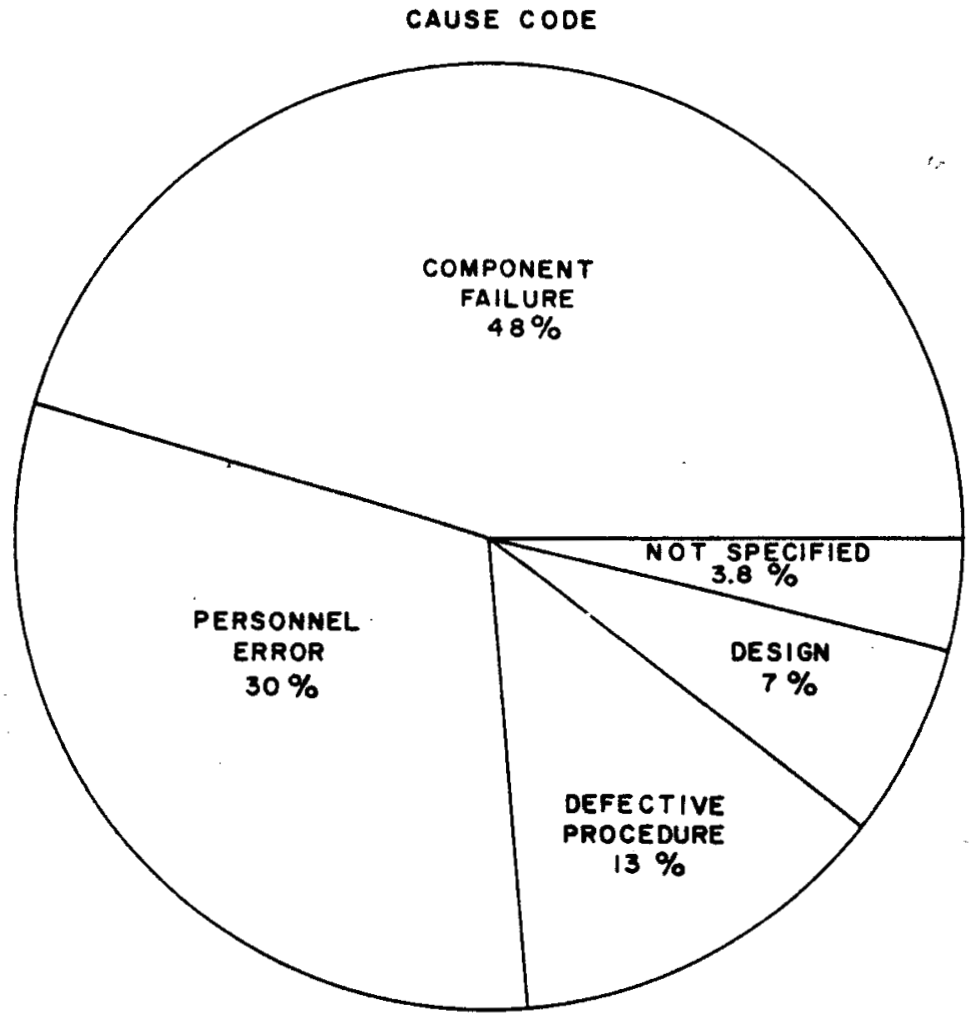

Figure 2.4a. Breakdown of the releases based on the cause code. 


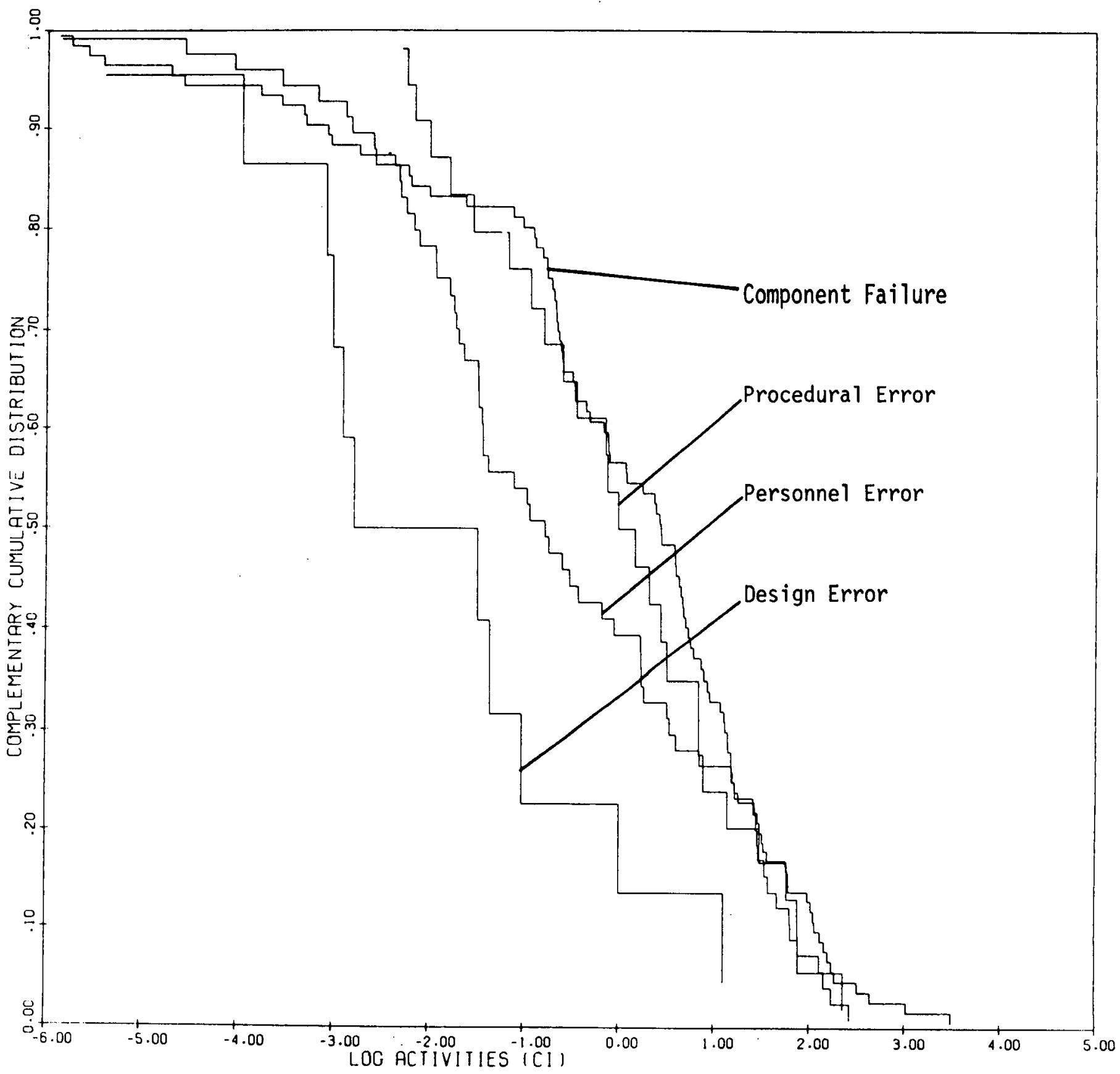

Figure 2.4b. Complementary cumulative distribution functions of the releases reported in the LER file for different cause codes. 


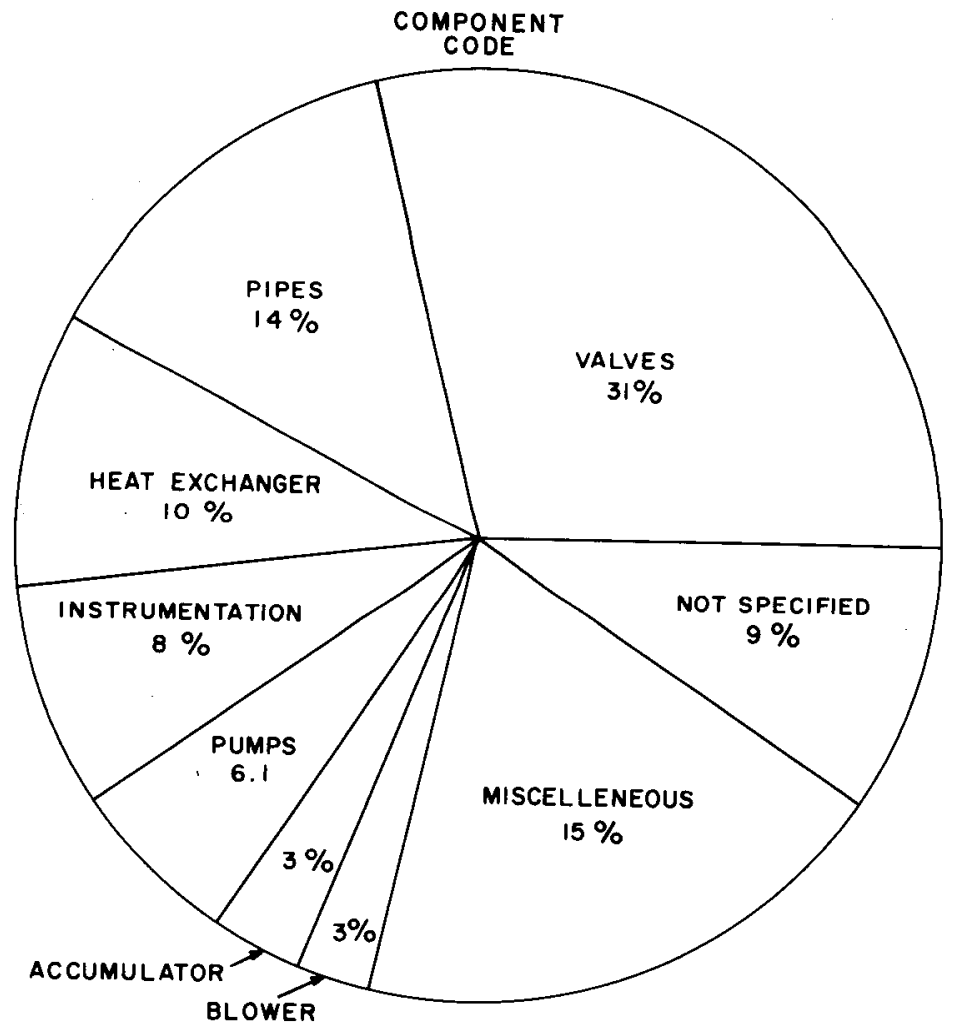

Figure 2.5a. Breakdown of the releases based on the component code. 


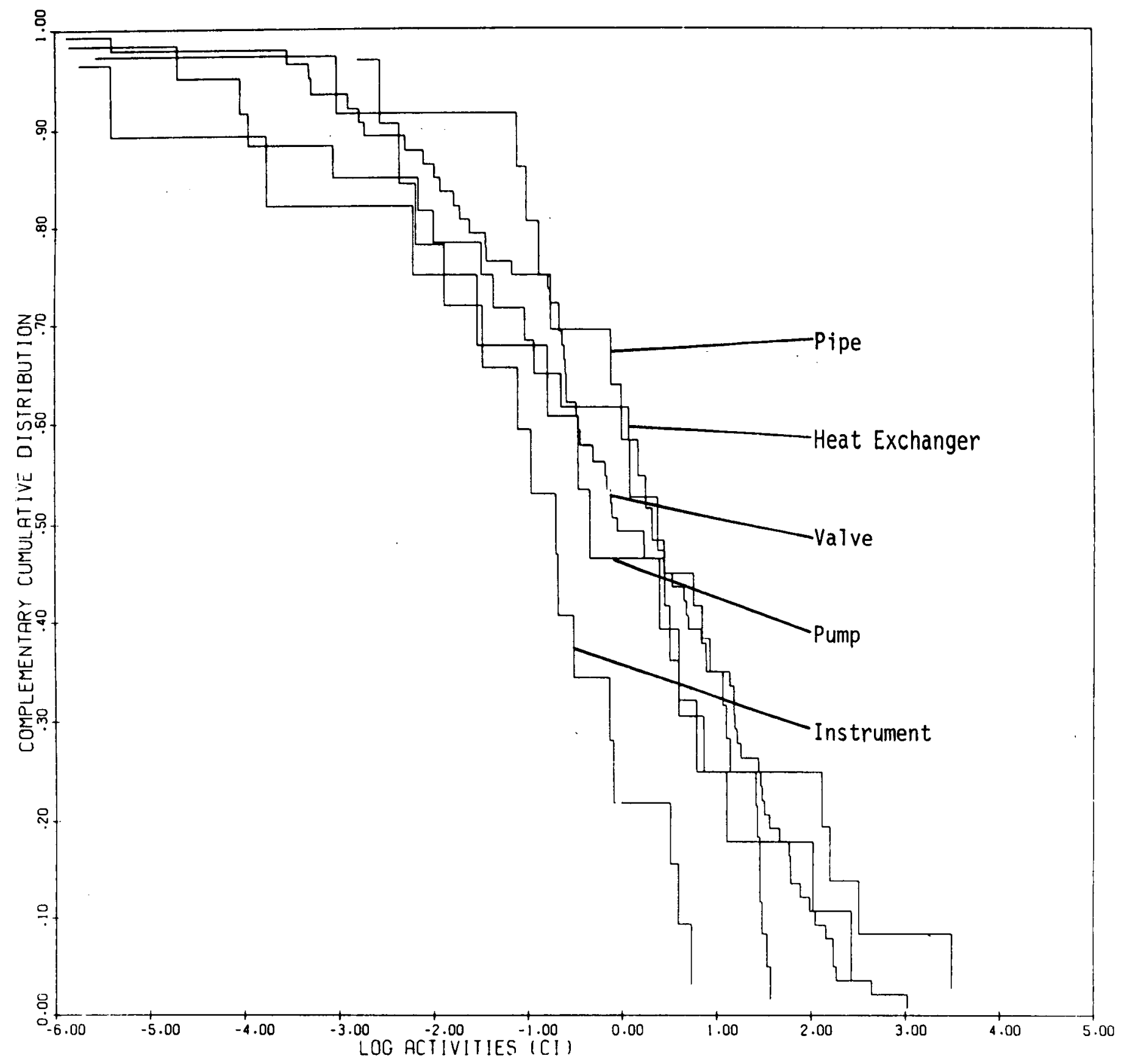

Figure 2.5b. Complementary cumulative distribution functions of the releases reported in the LER file for different components 


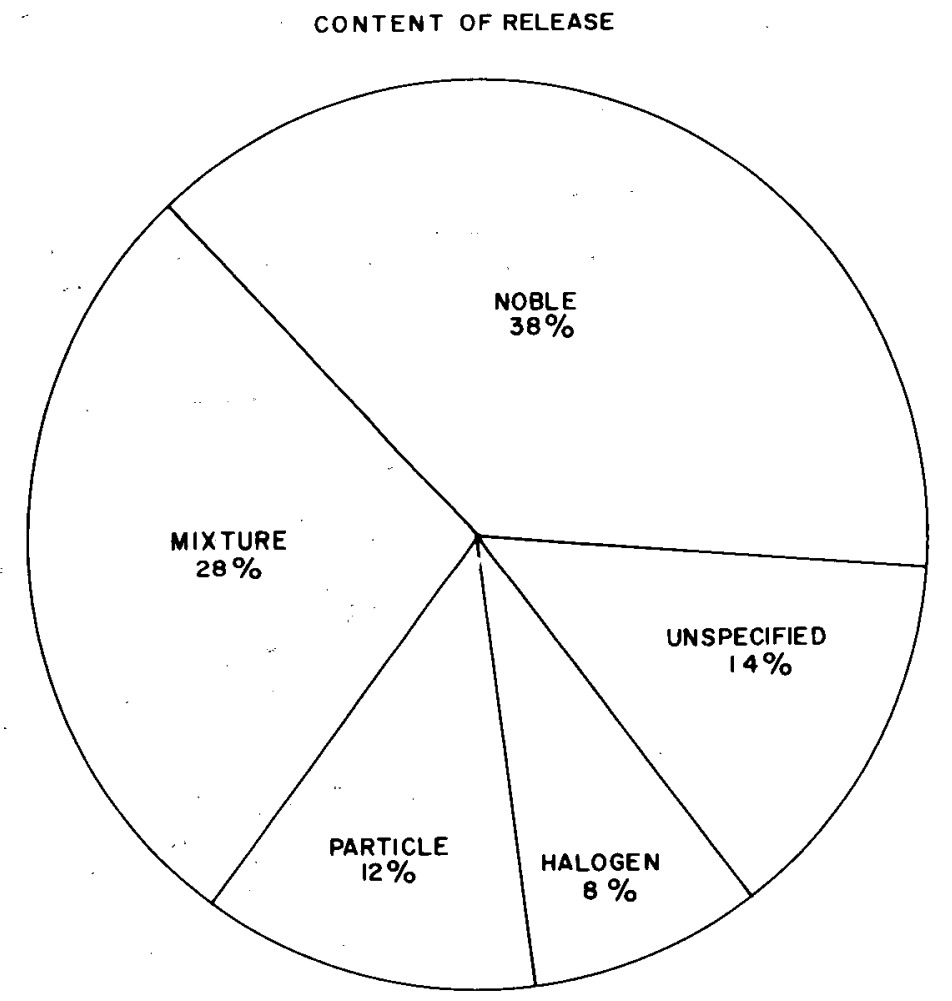

Figure 2.6a. Breakdown of the releases based on the form of the release. 


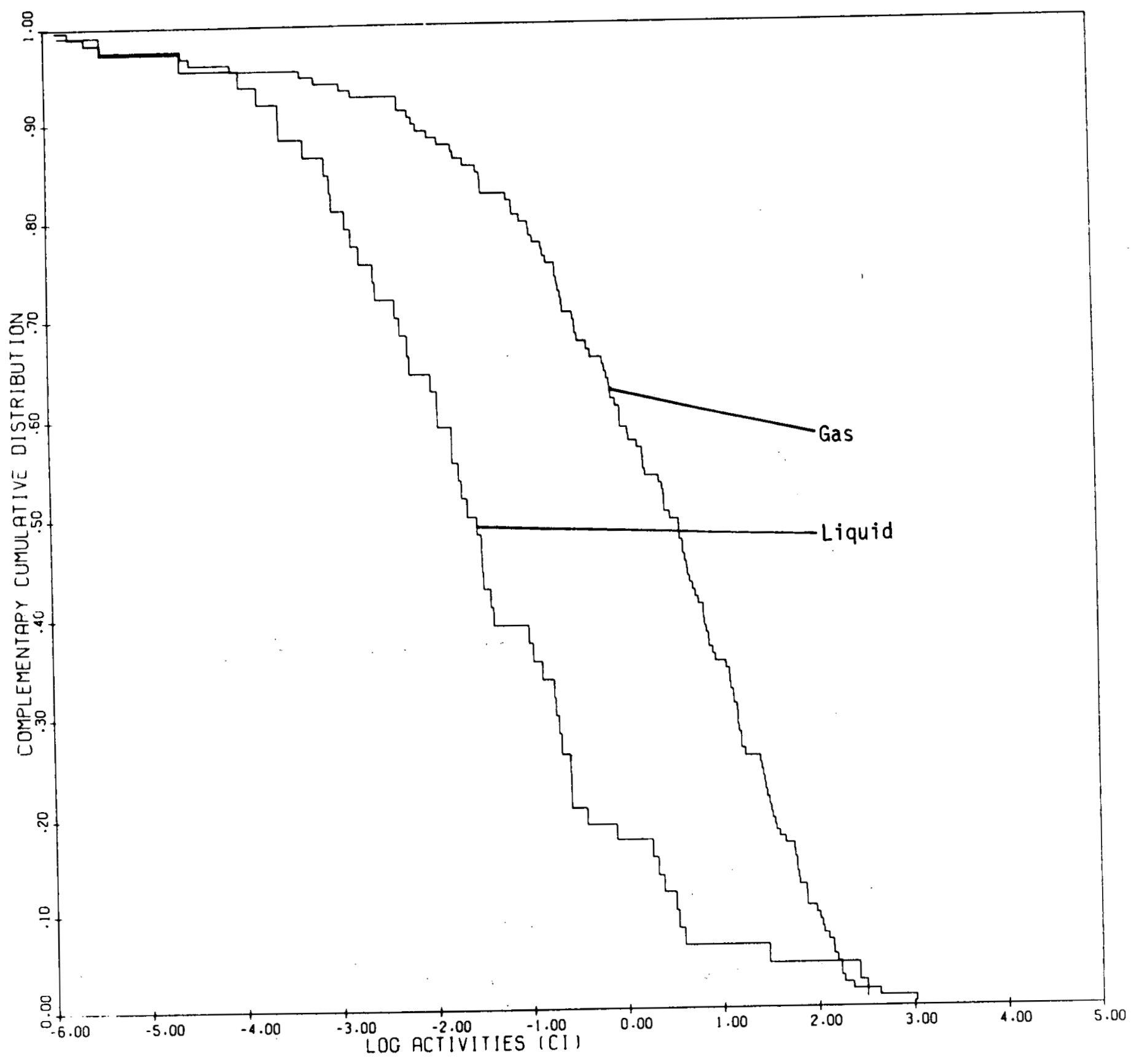

Figure 2.6b. Complementary cumulative distribution functions of the releases reported in the LER file for gaseous and liquid releases. 


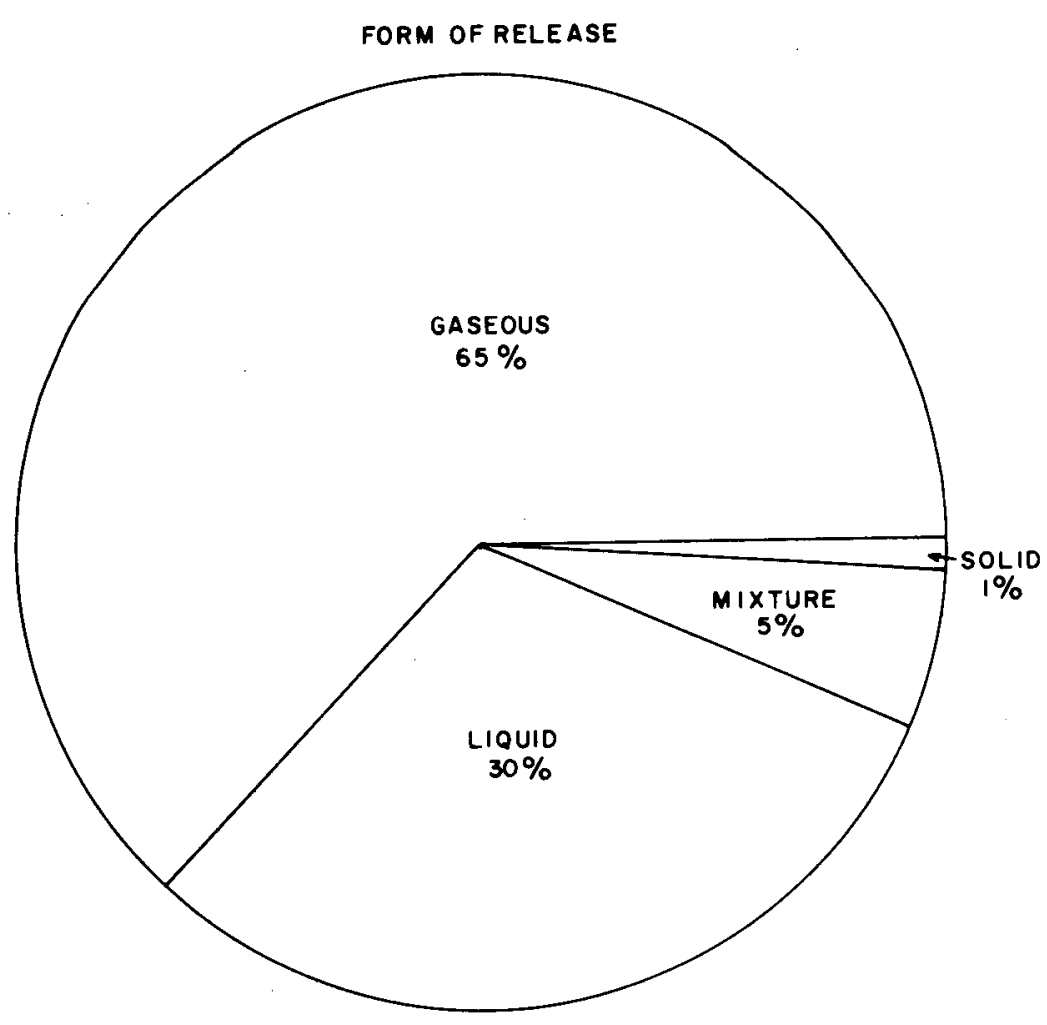

Figure 2.7a. Breakdown of the releases based on the content of the releases 


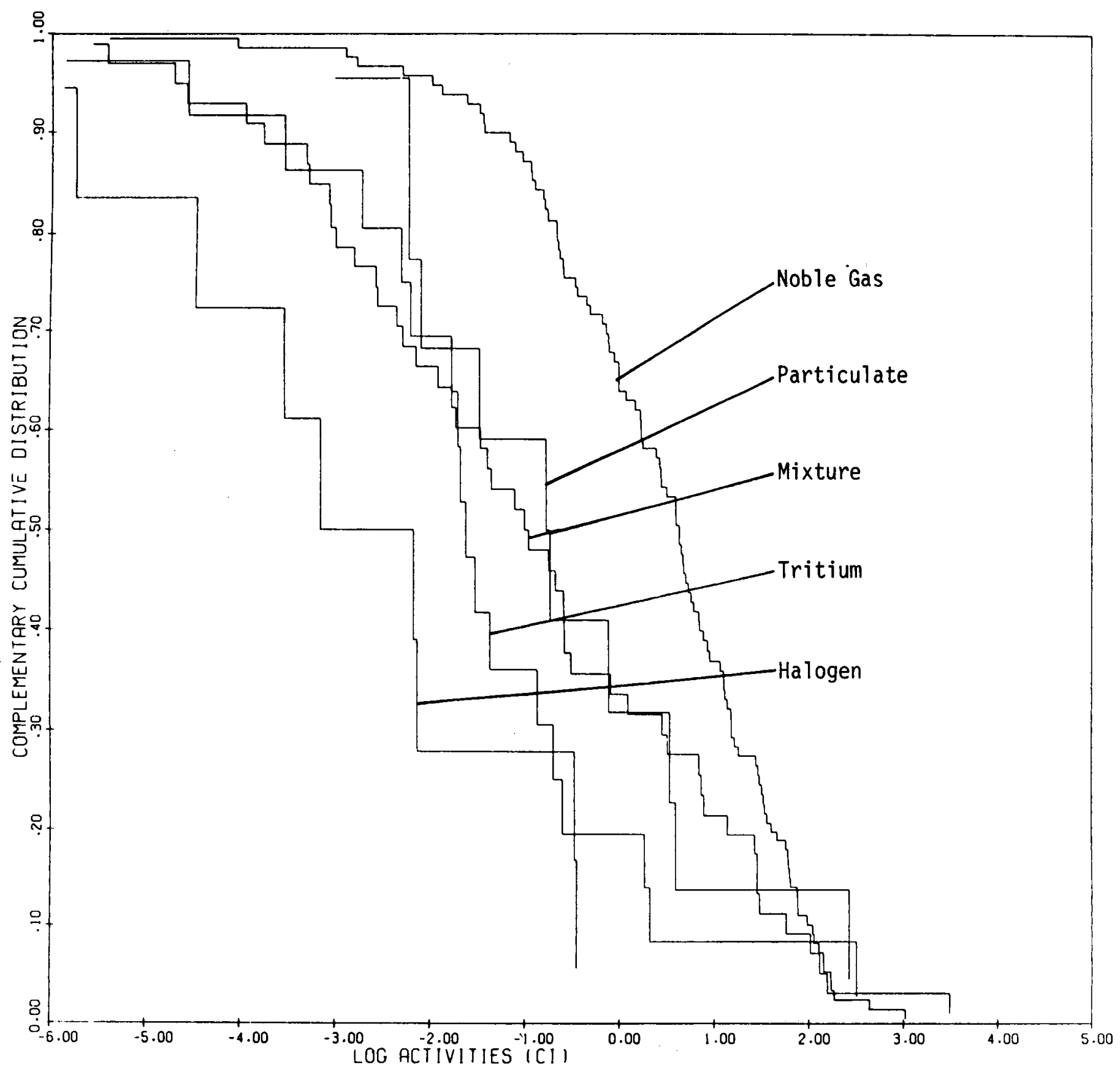

Figure 2.7b. Complementary cumulative distribution functions of the releases reported in the LER file for different contents 


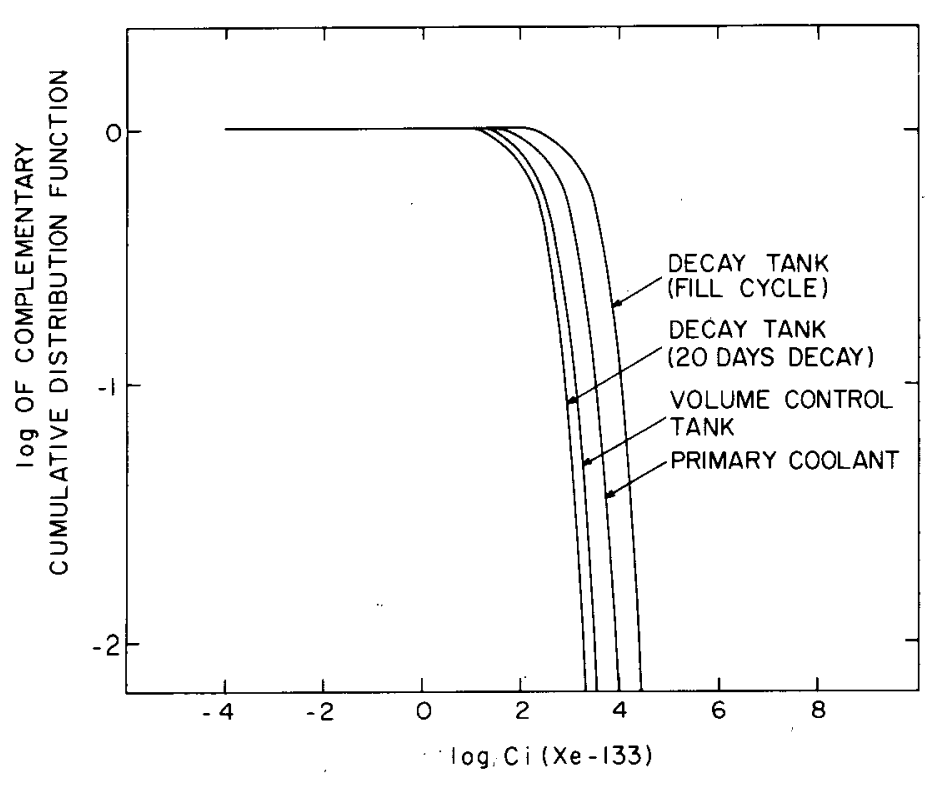

Figure 2.8a. Calculated CCF for Xe-133 in the gas decay tank, volume control tank and primary coolant

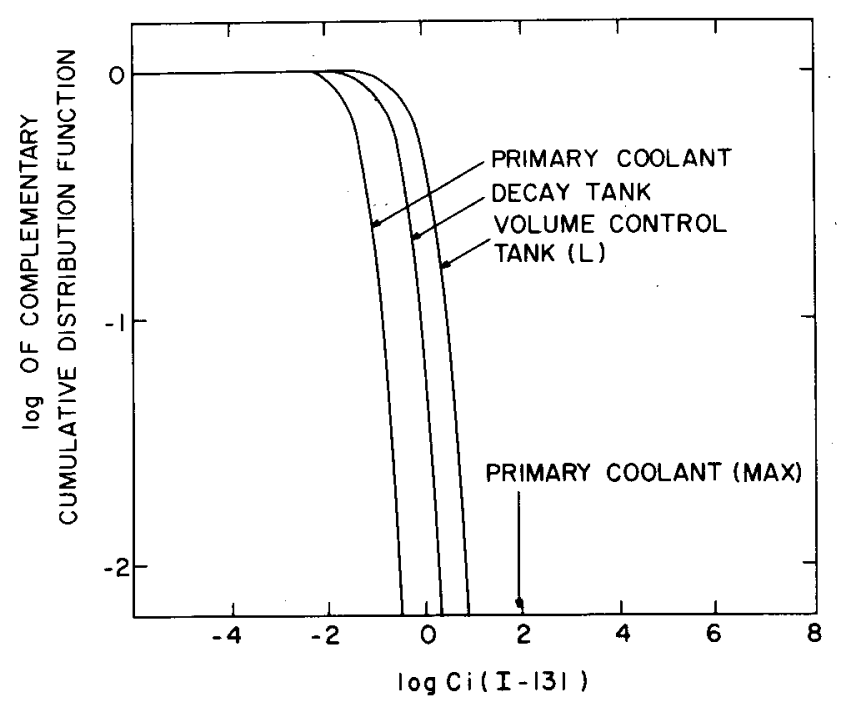

Figure 2.8b. Calculated CCF for 1-131 in the gas decay tank, volume control tank and primary coolant

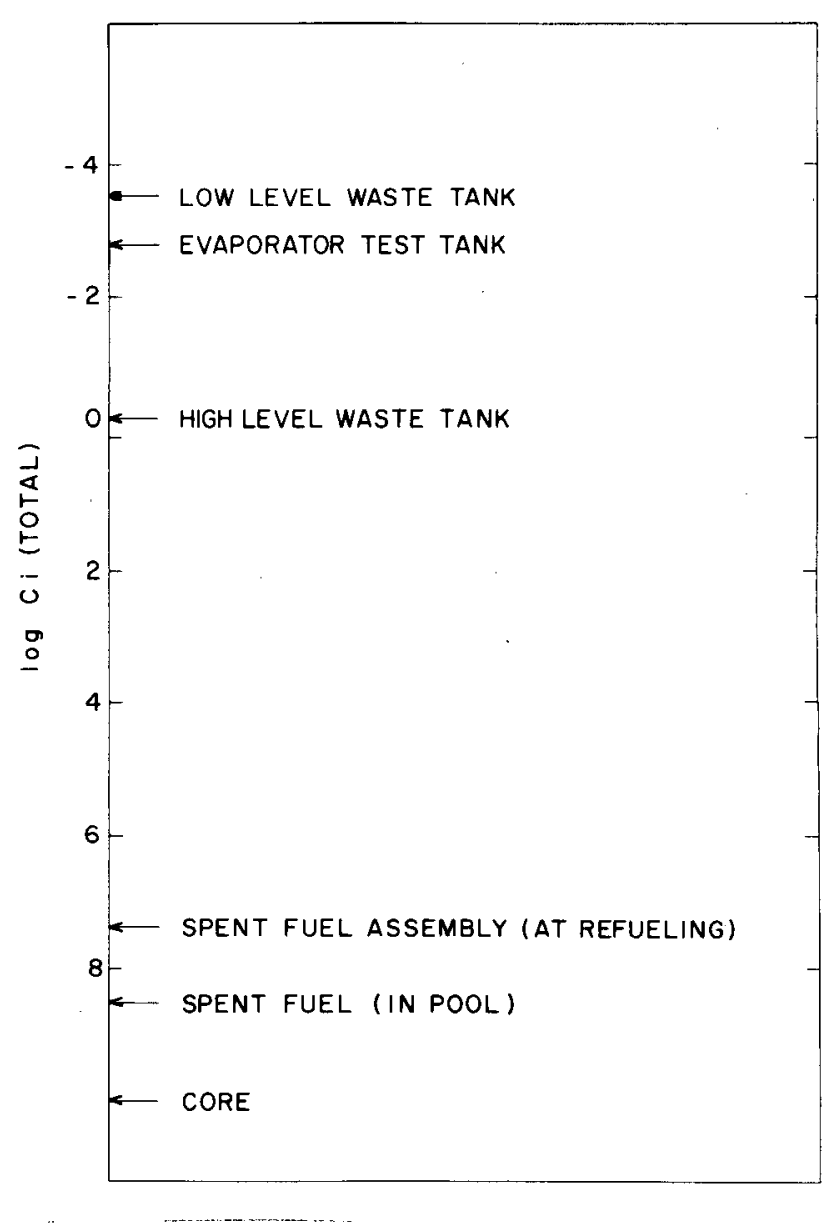

Figure 2.8c. Estimated total inventories of radionuclides in several components 


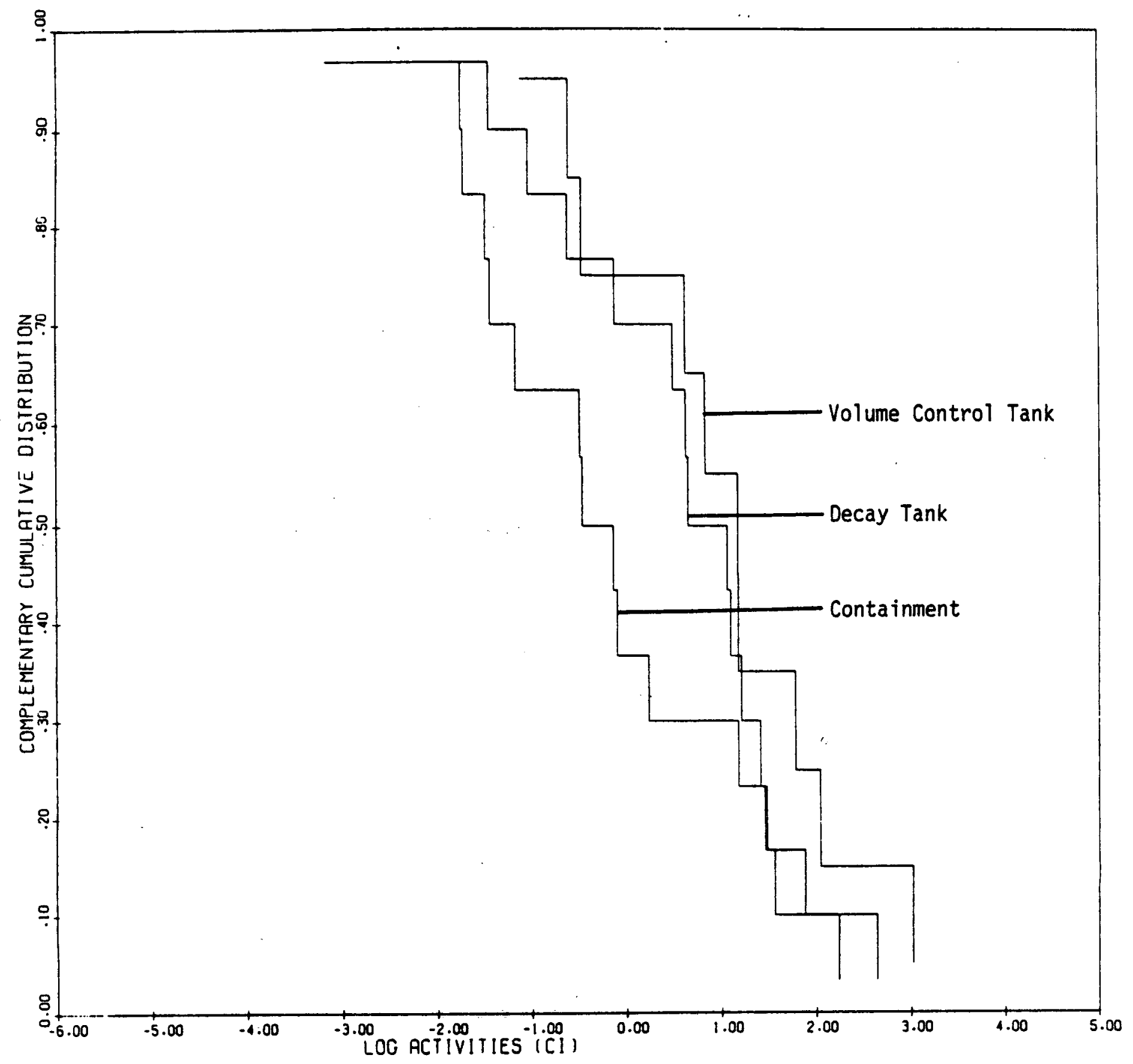

Figure 2.9a. Complementary cumulative distribution functions of the releases reported in the LER file in. which the source of the radioactivity was the volume control tank, decay tank or the containment. 


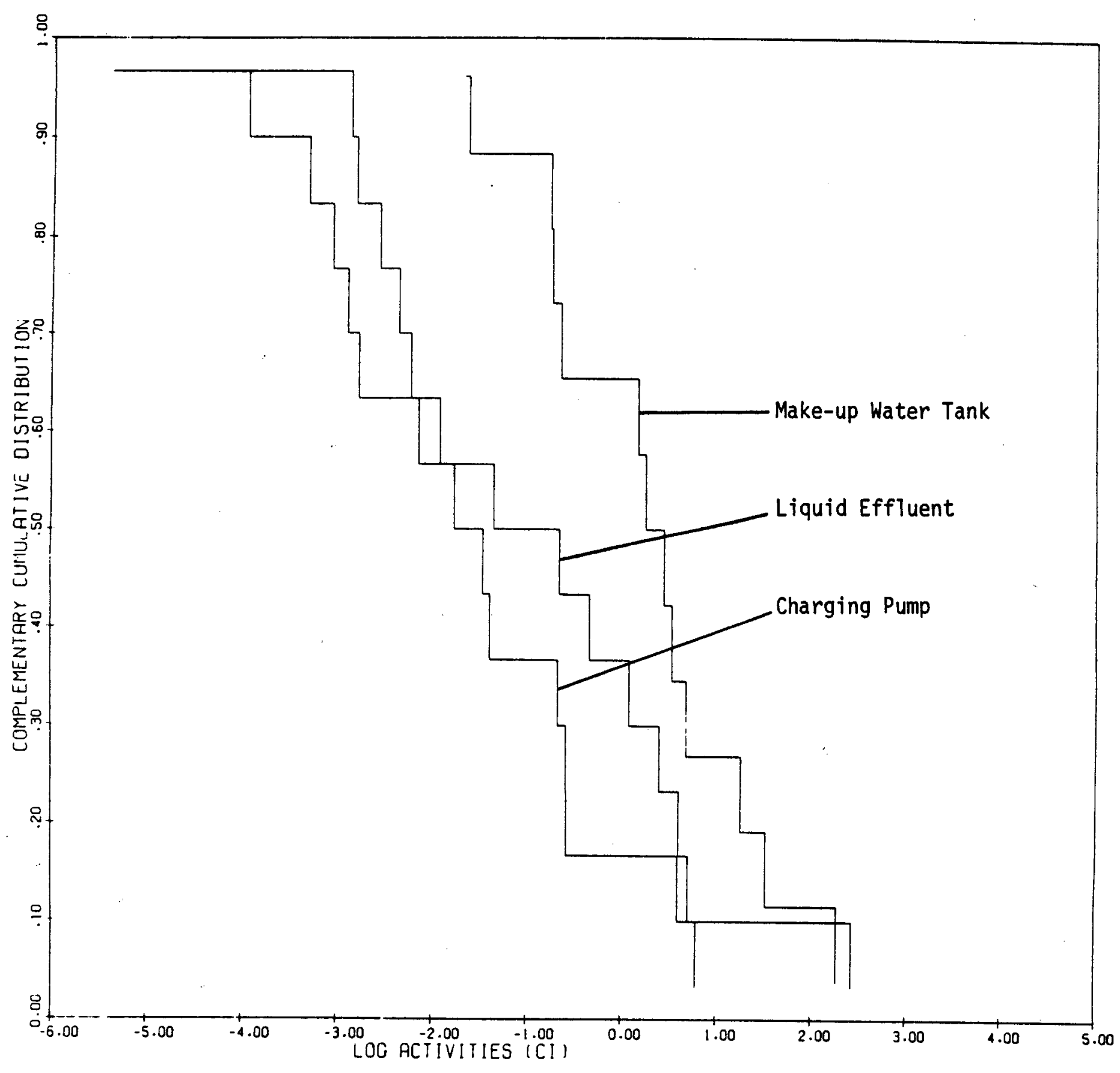

Figure 2.9b. Complementary cumulative distribution functions of the releases reported in the LER file in which the source of the radioactivity was the makeup water tank, the charging pump or liquid effluent 


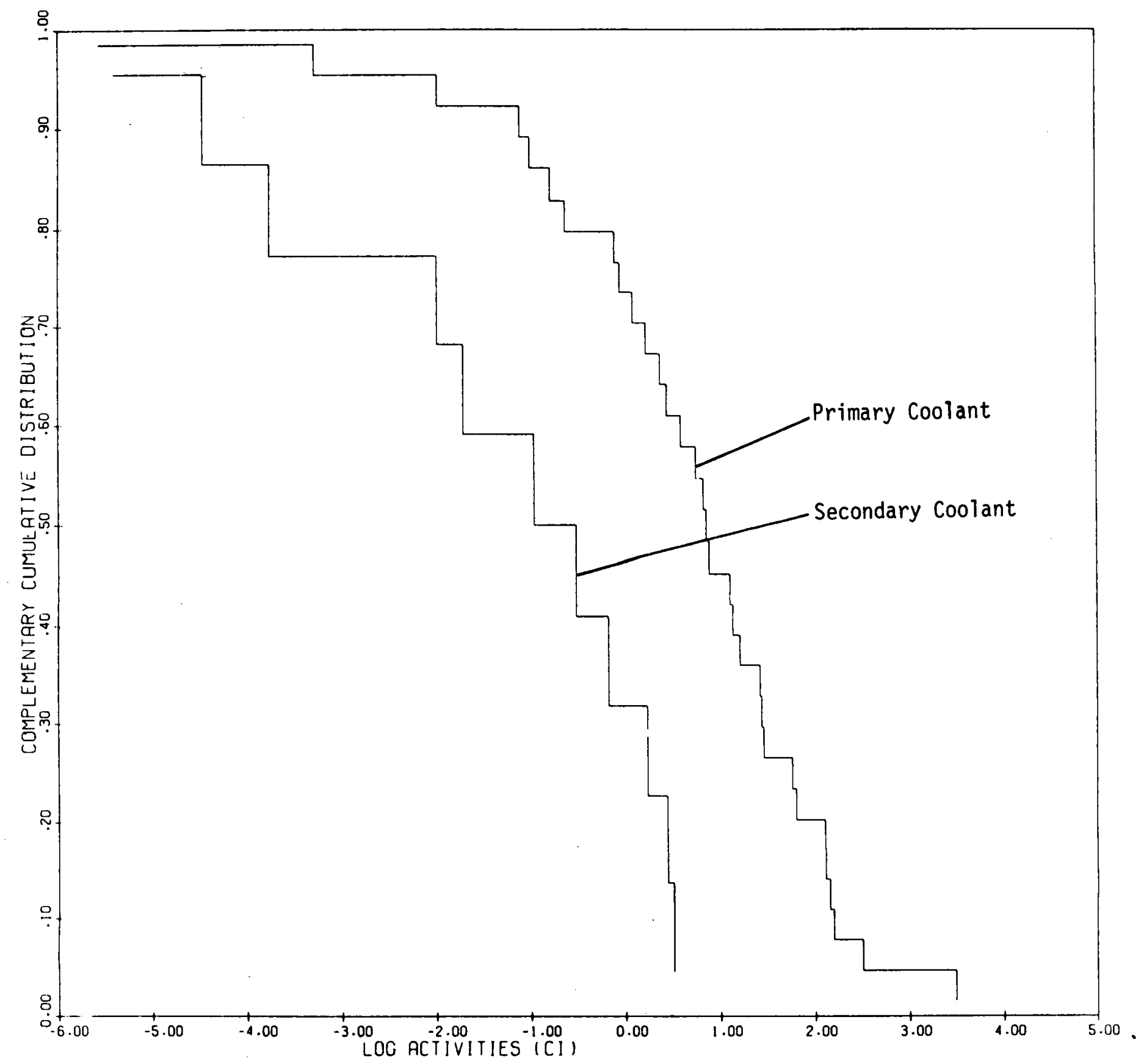

Figure 2.9c. Complementary cumulative distribution functions of the releases reported in the LER file in which the source of the radioactivity was primary or secondary coolant 


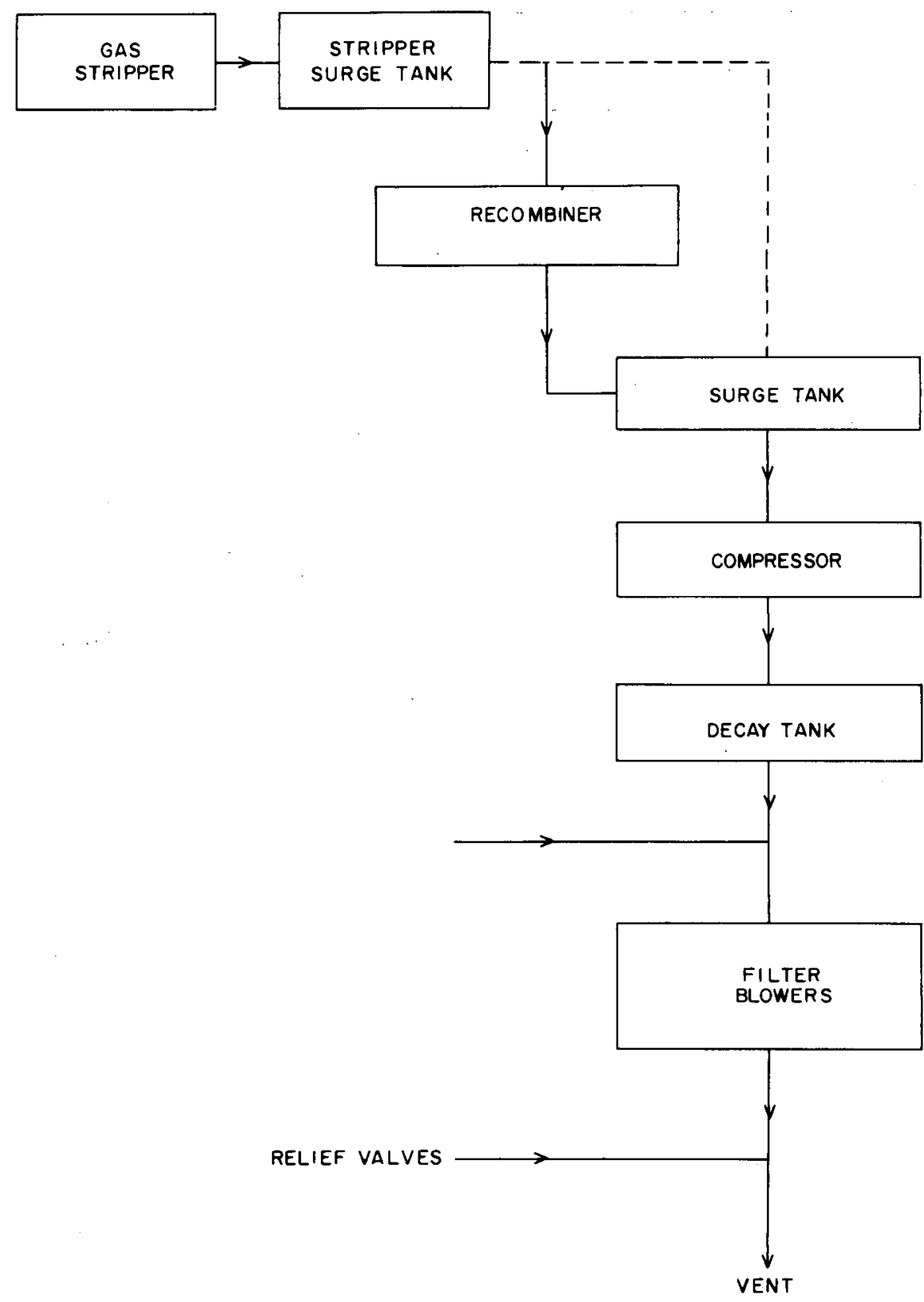

Figure 2.10. Schematic of the Surry gas waste management system 


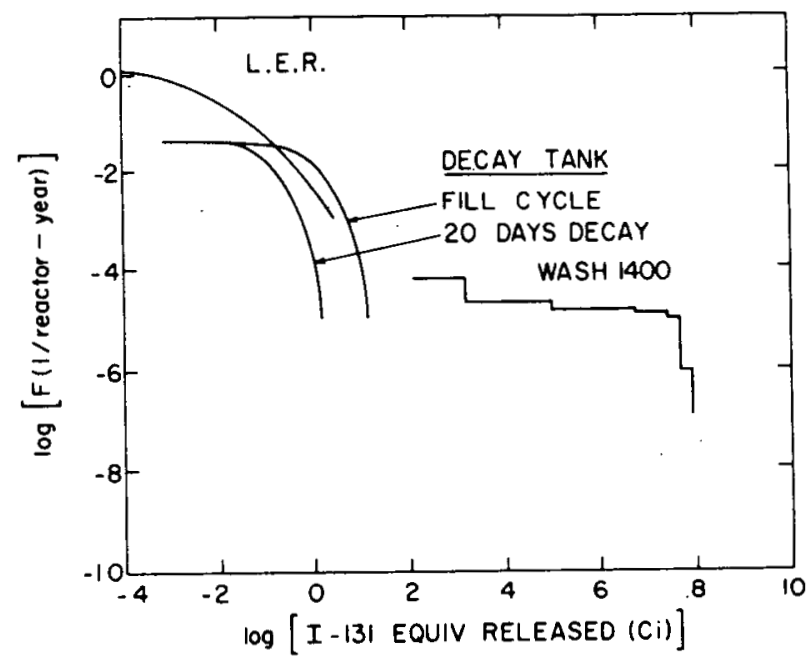

Figure 2.11. Calculated and observed CCF for releases from the waste gas decay tanks

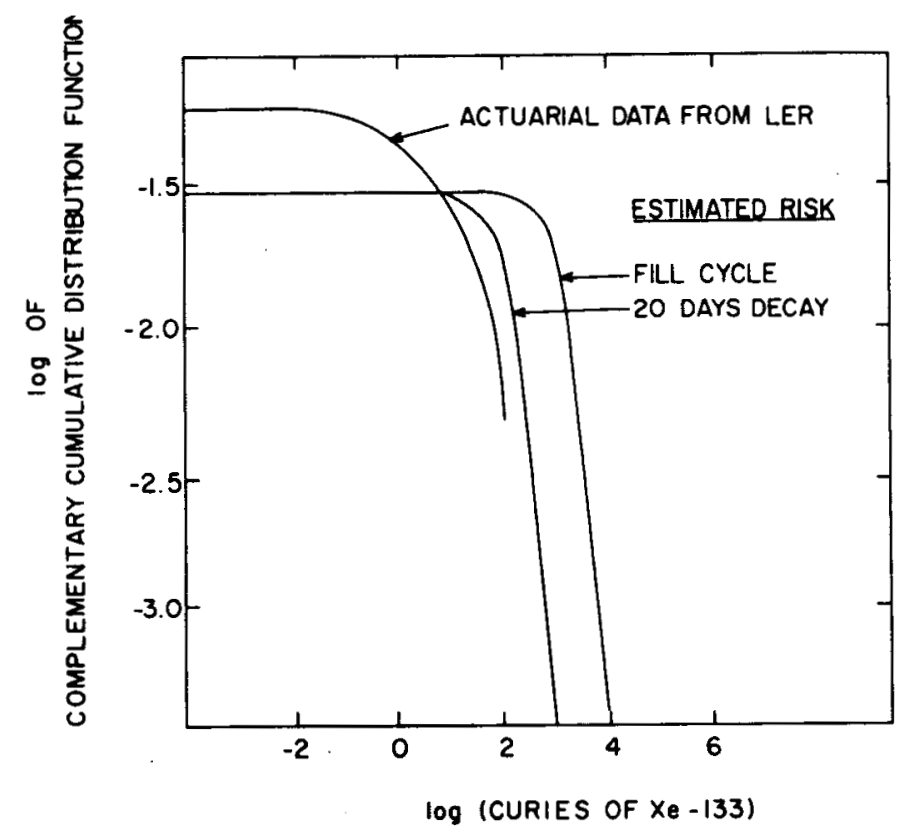

Figure 2.12. The weighted risk calculated for waste gas decay tanks compared to the LER data and the results reported in WASH-1400. 


\subsection{ENGINEERING ANALYSES FOR CLASS 3-8 ACCIDENTS}

In the previous section an actuarial approach was taken to quantify reactor risk in the low consequence-high probability portion of the risk spectrum. For accidents of lower probability, it is not possible to estimate risk directly from operational experience. In this region of the risk spectrum it becomes necessary to apply methods of engineering analysis to infer the risk as was done in WASH-1400 for Class 9 accidents. Although the risk that is determined in this manner does not involve the direct use of accident data, it is based upon the use of operational data to estimate the frequency of accident initiating events and the availability of systems. The methods of engineering analysis are used to combine these probabilities and to associate them with appropriate accident consequences.

The methods of engineering analysis used in this program are an extension of the methods developed in WASH-1400. Event trees are used to tabulate the large variety of accident sequences that can potentially succeed an initiating event. The probabilities of the branches on the event tree are determined by fault tree analysis or from the operational experience of components. An estimate of the consequences must then be made for each sequence. In WASH-1400 the branches on the event trees were treated in a binomial manner. A system was assumed to either operate or to fail. In general, this assumption is only an approximation to a range of potential operational modes. For example, a valve may fail to close upon demand and remain in the fully open position as in the binomial assumption or it may partially close with a spectrun of possible leak rates. In the analysis of Class 3-8 accidents it was found useful to develop a method of partial failure analysis which could be applied to 
systems which are better represented by a spectrum of performance modes. Because of the large number of accident sequences identified, it was necessary in this study to select only a limited subset for analysis. This was done by discriminating according to either sequence probability or consequence. Since the frequency of core meltdown accidents was estimated in WASH-1400 to be in the neighborhood of $5 \times 10^{-5}$ per reactor year, it is clear that any Class 3-8 accident which is estimated to have a lower probability would have a negligible effect on the risk curve because of the much smaller releases of radionuclides in these accidents. A discrimination level of $1 \times 10^{-5}$ per reactor year has been used. Similarly, to select the sequence to be analyzed, accidents involving the release of less than $1 \mathrm{Ci}$ of radionuclides are not of particular interest because this part of the risk spectrum appears to be adequately characterized by actuarial data.

In order to help identify the accidents to be investigated in some detail, two week-long workshops were conducted. It should be emphasized that no claim of completeness is made in this study. It is believed that some of the most important accident sequences have been examined and that a better insight into the contribution of Class 3-8 accidents to accident risk has been obtained as a result. In Section 4 the results of a complementary survey of the plant are described which provide some degree of assurance that most key accident sequences have been identified.

In this section of the report, engineering analyses will be described for a number of accident sequences. The accident sequences are organized according to the Class 1-9 categorization of 10CFR50. No accidents which would be categorized Class 1 or Class 2 are considered by definition of the scope of the effort. In addition, the transient initiated by a loss of feedwater is 
briefly described in order to estimate roughly the frequency of the events. Class 3 accidents were treated in Section 2 of this report using actuarial data from the LER files. The calculations of the consequences of accident sequences are based primarily on data given in the Surry FSAR, the Gale Report, 7 and occasionally on RESAR-3S, 8 if the relevant information for Surry is not available.

\subsection{PARTIAL FAILURE ANALYSIS}

The analysis in WASH-1400 used fault and event trees in which there were two potential outcomes for each event. In this report a special technique termed "Partial Failure Analysis" has been developed to treat accidents in which each event is better characterized by a spectrum of operational modes. The course of a specific accident at a given time and place is deterministic. However, the outcome of the accident will depend on the local conditions. Thus the predicted consequences of a hypothetical accident scenario cover a spectrum of possibilities. It is useful to distinguish between the variation which is inherent in the problem and that which is due to our ignorance of the physical processes involved. The former uncertainty is random in that different states could be encountered depending on the specific timing or circumstances of the accident. The latter uncertainty is systematic and may be expressed as a degree of confidence in the results of our calculation, that is as error bands. Examples of partial failure analysis are presented in Appendix I for the case of a fuel handling accident in the spent fuel storage pool and in Appendix $J$ for the case of loss of cooling for the spent fuel storage pool.

The general approach involves the following steps: 
1. Develop a simple relationship between the consequences of an accident and the variables or parameters describing the system, e.g.

$$
C=f\left(x_{1}\right) g\left(x_{2}\right) h\left(x_{3}\right) \text {. }
$$

2. Identify the sources of uncertainty. We will use the term 'variables' for those sources regarded as random and the term 'parameters' for those sources regarded as systematic.

3. Develop probability density functions for those variables that are random in nature.

4. For the remaining parameters, quantify the systematic uncertainty interval that exists as the result of ignorance. Typically, 90 percent confidence intervals are used, although in some cases subjective judgment must be applied. Parameterize the probability density function for each parameter in such a manner that the range of systematic uncertainty is spanned by varying the parameters. This probability should be interpreted in a Bayesian sense. (5)

5. Randomly select a set of parameters that characterize all of the density functions. For this chosen set develop a complementary cumulative distribution function for accident consequences. If the density functions and the relationship between the variables and the consequences are sufficiently simple, this can be done analytically. In general, the CCF can be obtained by Monte Carlo(6) analysis.

7. Perform a large number of calculations in the manner described in the previous step. The shape and centrality of each of the CCF's obtained in Step 5 above are defined by the variables which exhibit random variation." From the group of CCF's obtained in Step 6 an average or expected curve can be obtained and error bands can be calculated which describe the uncertainty which arises from ignorance. 


\subsection{FISSION PRODUCTS TO PRIMARY AND SECONDARY SYSTEMS (CIASS 5 )}

Three subcategories of Class 5 accidents are defined in the 10CFR50 classification scheme:

1. Fuel cladding defects and steam generator leaks

2. Off-design transients that induce fuel failure above those expected and steam generator leak

3. Steam generator tube rupture.

Since fuel cladding defects and steam generator leaks are encountered routinely in operation, the first of these subcategories can be considered as belonging to routine releases as opposed to accidental releases. In addition to the second subcategory, this study includes transient events which do not induce major fuel failure. Accidents initiated by the rupture of a steam generator tube are evaluated in this class of accidents. The possibility of steam generator tube ruptures resulting from the loads induced by steam line breaks or loss-of-coolant accidents are considered as Class 8 accidents. In view of the importance of transients initiated by loss of feedwater, the event trees for these accidents are discussed separately in Section 3.6.

\subsubsection{Iransients}

A number of transient accident events, such as loss of flow from a primary system pump, are analyzed in safety analysis reports to demonstrate that departure from nucleate boiling and associated fuel failures will not result. If an orderly plant shutdown follows these events, some release of radioactivity will occur from the condenser air ejector but the release will be within the limits of normal releases. 
Some transient events can be hypothesized which would induce fuel failures such as the development of flow blockages or core power maldistributions. Accidents of this type are analyzed in enviromental reports under the assumption that 0.12 percent of the core inventory of noble gas and halogens is released to the primary coolant. This release would correspond approximately to the gap inventory of 1 percent of the core or one to two assemblies. In comparison to the doses calculated for other Class $3-8$ accidents, the doses from this type of accident are found to be relatively small. These accidents have not been considered further in this study. of course some possibility exists for the compounding of accidents which induce fuel failures with other independent events which could lead to larger releases. Since operating history indicates that accidents which lead to fuel failure are infrequent, the combined probability of these accidents with other failures should be less than the $10^{-5} \mathrm{yr}^{-1}$ discrimination level used in this study.

One type of transient initiator, loss-of-offsite power, which occurs with relatively high frequency, simultaneously leads to the disabling of systems that nomally control the release of radionuclides to the environnent. Figure 3.1 illustrates an event tree for three potential subsequences that could result from a loss-of-offsite power. The a subsequence is the most probable outcome of the accident and is the one analyzed in this Section. The $\beta$ subsequence involves a failure in the secondary system subsequent to loss-ofoffsite power. The example that has been considered is the failure of a pressure relief valve to reseat. The release of radioactivity in this subsequence would be greater than for the a subsequence but the probability is a factor of 10-2 sinaller. (1) The $\gamma$ subsequence is the anticipated transient without 
scram accident which has been evaluated in some detail in other references and thus, will not be further pursued at this level. The principal question about the $\gamma$ accident is whether or not meltdown of the core ensues. If meltdown does not occur, the probability of this accident is too small to be of interest as a class $3-8$ event.

\subsubsection{Loss-of-0ffsite Power with No Compounding Failures}

In this accident sequence it is assumed that the reactor is operating at full power at the time of loss-of-offsite power. Turbine trip rapidly follows the loss of load from the generator. The condenser circulating water pumps, which are driven by offsite power, shut down. Steam bypass from the common steam header to the condensers is prevented to protect the condensers from damage. Excess steam pressure in the secondary coolant system is relieved to the atmosphere by a controlled steam dump via the main steam safety valves. After approximately $1 / 2$ hour, sensible and nuclear heat in excess of plant auxiliary requirements is released to the atmosphere by the decay heat release control valve operated from the main control room. Steam flow is controlled as function of the reactor coolant temperature and is reduced as rapidly as the reactor control system can reduce core power. Following the initial automatic period of the steam dump during power cutback, the decay heat release control valve is adjusted to provide control over the longer term heat balance requirements.

The amount of radioactivity released following a loss-of-offsite power will vary primarily as a function of the percent of failed fuel during operation, the primary to secondary coolant system leak rate, the repair time for the return to offsite power and the time since the previous shutdown, which 
affects iodine spiking if the previous shutdown was recent. A partial failure analys is was performed for this accident in which each of these factors was treated probabilistically. The probability density functions were developed as follows:

1. Failed fuel distribution. Based upon the GALE report(7) a median value of 0.12 percent failed fuel was obtained. The distribution was assumed to be log normal with a 95 percent probability of less than 1 percent failed fuel.

2. Primary to secondary leak rate. Figure 3.2 shows the complementary cumulative distribution for leak rate which was obtained from the GALE report. (7) The probability of a finite leak size is taken as 0.36 from the same report. A constraint of less than $10 \mathrm{gpm}$ was placed on the distribution, based on Surry Tech Spec leak rate limits.

3. Repair time for offsite power. The cumulative distribution for repair time illustrated in Figure 3.3 was taken from WASH-1400. (1)

4. Time since last shutdown $(\tau)$. It was assumed that events leading to iodine spiking occur with a frequency of $\lambda_{S}=10^{-3} \mathrm{hr}^{-1}$ which is approximately the rate at which turbine trips have experienced. The density function for the time since last shutdown equals $\lambda_{S} e^{-\lambda}(\tau)$. A computer code was written to perform the partial failure analysis for the loss-of-offsite power accident. The mathematical analys is is described in detail in Appendix $F$. The following steps were performed in the analysis.

1. Random selection was made of a leak rate.

2. Random selection was made of a failed fuel percentage.

3. Secondary activity was calculated assuming direct proportionality with leak rate and failed fuel percentage. The proportionality constant 


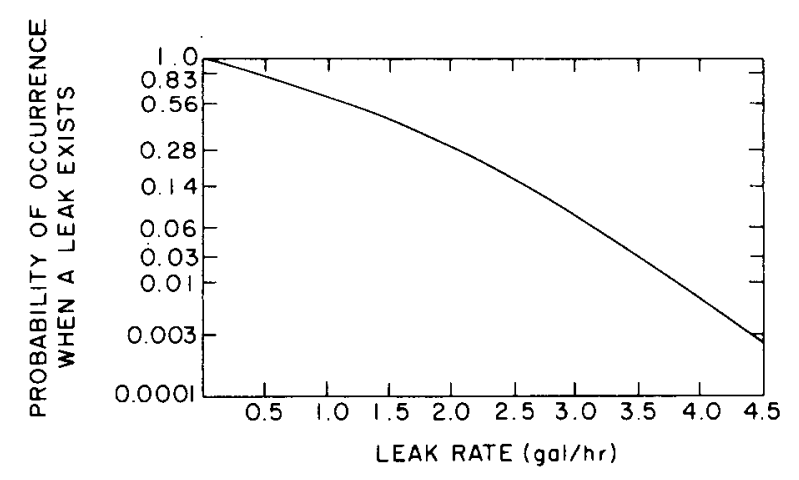

Figure 3.2. Probability distribution of primary to secondary leak rate

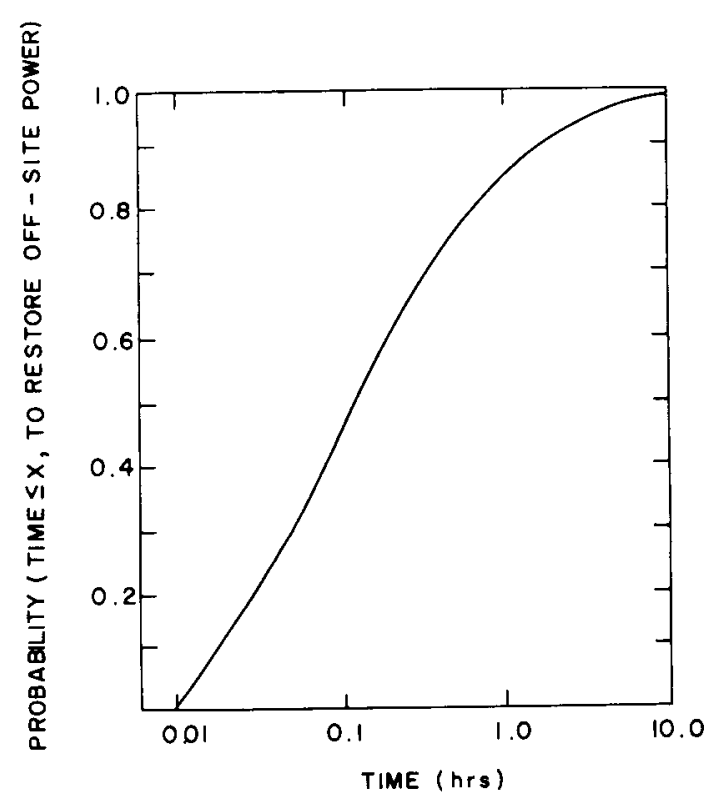

Figure 3.3. CCF for time to restore offsite power 
was based upon data in RESAR 35 analysis(8). A limit of 9Ci total secondary inventory was assumed.

4. Random selection was made of the time since last shutdown.

5. Random selection was made of the repair time for loss-of-offsite power.

6. The mass of steam released to the atmosphere was calculated using the repair time and Figure 3.4 which describes cumulative release of steam versus time. This figure was developed from data in RESAR 3S(8).

7. The quantity of radionuclides released was calculated according to

$$
Q=M_{S} a_{S} S P
$$

where

$$
\begin{aligned}
M_{S} & =\text { mass of steam released } \\
a_{S} & =\text { secondary concentration } \\
S & =\text { spiking multiple } \\
P & =\text { partition factor } \\
Q & =\text { quantity released }
\end{aligned}
$$

The spiking factor, which is a function of the time since last shutdown, was determined according to Spiking Model A described in Appendix F. A partition factor of 0.1 was assumed for iodine.

8. A histogram of release was developed by placing the result of each of the 10,000 Monte Carlo histories which were run into a bin according to the magnitude of the release.

9. A complementary cumulative distribution curve was developed from the histogram.

In the partial failure analysis performed for this accident sequence, uncertainty bands associated with systematic error were not developed, since the final results indicate that this accident scenario is relatively unimportant. 


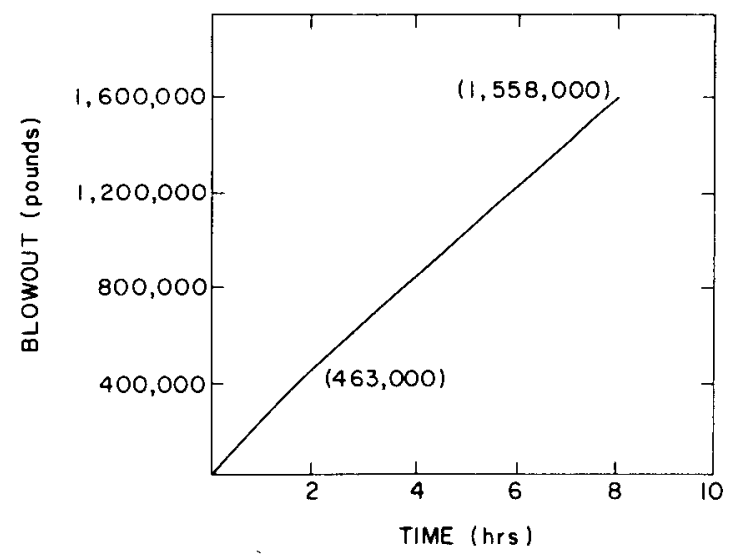

Figure 3.4. Cumulative release of steam to the atmosphere versus time

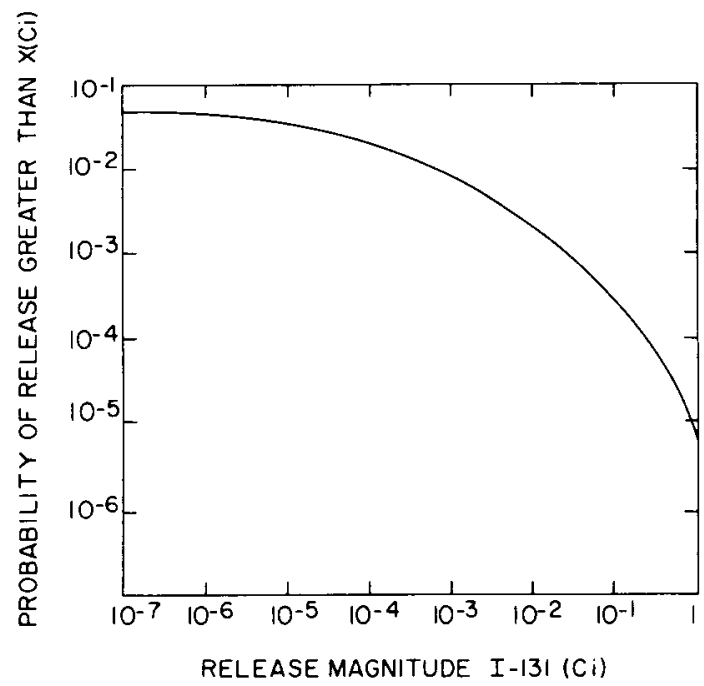

Figure 3.5. Calculated CCF for loss of offsite power accident

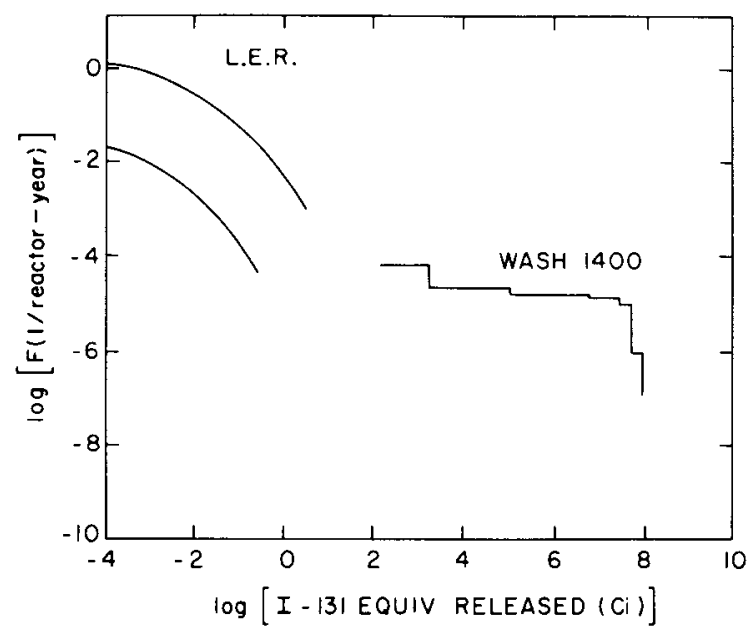

Figure 3.6. The weighted risk calculated for loss of offsite power compared to the LER data and the results reported in WASH-1400 
Results

The complementary cumulative distribution curves for the release of I-131 is illustrated in Figure 3.5. In some respects the distribution curve for loss-of-offsite power is generic in that the frequencies of loss of power and of the repair time are not plant specific but represent previous U.S. experience. In Figure 3.6 the weighted risk of all iodine nuclides for lossof-offsite power is compared with the results of LER analysis at the low consequence end and with Class 9 accidents at the high consequence end of the risk curve. It can be seen that the loss-of-offsite power curve falls within the region defined by available data.

\subsubsection{Steam Generator Tube Rupture}

In this class of accidents the rupture of a steam generator tube will be considered as an initiating event. Usually this accident is assumed to occur spontaneously while the reactor is operating at full power, although it should be recognized that the event may be as likely to occur as the result of transient loads during a change in power. The consequences of the accident being initiated under transient conditions could be less than from full power operation or could be greater due to iodine spiking. Since tube degradation and even through-wall cracking of tubes have been common in reactor experience, the potential for a tube rupture does exist. Whether or not a crack will leak or run depends on its length. Some discussion of the mechanics of tube rupture is presented in Appendix $M$ in regards to the loads imposed in steam line breaks. For the purposes of this study the probability of a steam 
generator tube rupture has been taken as $10^{-2} \mathrm{yr}^{-1}$ based upon a very 1 arge leak that occurred at Point Beach in 1975. Whether or not this leak qualifies as a tube rupture is somewhat questionable, however, and the probability is believed to be conservative.

In Figure 3.7 an event tree is presented for the steam generator tube rupture accident in which three possible compounding failures are evaluated:

Subsequence a has not been evaluated because it would be expected to have relatively small consequences. In this accident the radioactivity would be released through the air ejector of the condenser. The release of iodine is limited by the relatively small partition coefficient for iodine at the temperature of the condensate.

Sequence $\beta$ involves compounding failures in the secondary system and may be significant because they could lead to greater releases. Possible secondary failures considered are:

1. Turbine bypass valve fails to open

2. Water level in faulty steam generator rises into main steam line leading to pipe failure.

3. A relief or safety valve opens on the secondary side and fails to close.

Sequence, $\gamma$, is analyzed in this section of the report. The failure rate of the electric power grid upon challenge is estimated to be $10^{-3}$ in WASH1400.(1) In the event of loss-of-offsite power, steam can no longer be directed to the condenser and the secondary pressure relief valves will lift leading to a direct release of radioactivity.

Sequence $\delta$ involves failure of the reactor to shut down following the rupture. Since the failure rate of reactor trip (RT) is quite low, estimated as 


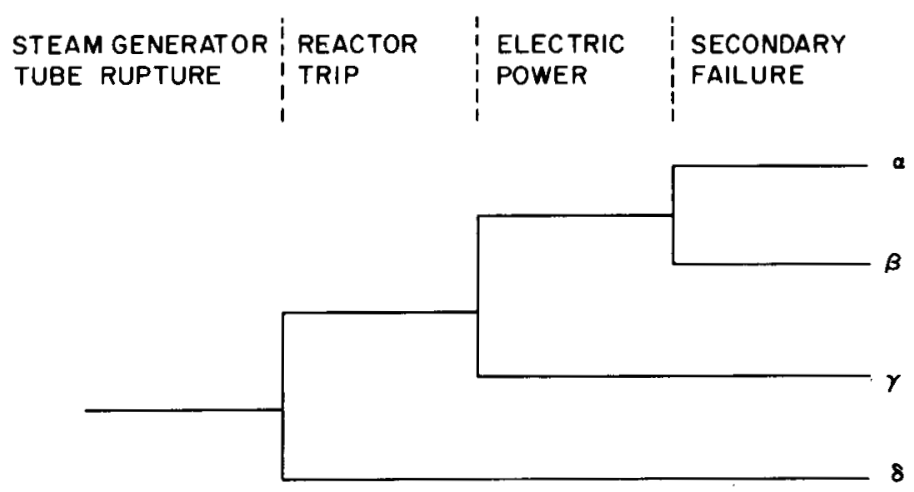

Figure 3.7. Event tree for steam generator tube rupture accident 
$10^{-4}$ in WASH-1400, the probability of this subsequence is small enough to be ignored in this study.

The consequences of each of these possible failures would be larger than for the a sequence. The turbine bypass valve failure $\left(10^{-3}-10^{-4}\right)$ is sufficiently improbable that combined with the initiating event it was felt that this sequence could be ignored. The consequences of a tube rupture with subsequent failure of a steam line would be very similar to the consequences of the case where the initiating event is a steam line break and the compounding failure is a steam generator tube rupture. This accident is analyzed in Section 3.5.3.1. The likelihood of the failure of a steam line following a tube rupture is difficult to assess because it involves the ability of the operator to diagnose and adequately control the affected stearn generator prior to overflow of secondary water into the steam line. The response of the steam line in the event of overflow has not been analyzed in this study. Since the probability of the accident evaluated in Section 3.5.3.1 is relatively high, it was not considered necessary to consider this possible subsequence in detail. he probability of the failure of a relief valve to reseat adequately following operation was evaluated in WASH-1400(1) as being relatively high, $10^{-2}$ per operation. It is not expected that the valves would operate, however, if the response to the accident is as anticipated. The consequences of this sequence would be similar to but potentially greater than the accident with loss-of-offsite power. 
3.2.2.1 Steam Generator Tube Rupture Compounded With Loss-of-Offsite Power

In the event of a tube rupture in a steam generator, primary system fluid will flow into the secondary side of the affected steam generator. It is claimed in the Safety Analys is Report for Surry that the operator can recognize the type of accident, identify the faulty steam generator and isolate it within 30 minutes. The Surry plant is actually not typical of PWRs in response to this accident in that it has isolation valves in the primary loops which can be used to isolate the affected steam generator on the primary side. In the more typical case, the primary system pressure must be reduced below the secondary so that the steam line can be closed on the damaged stean generator. This operation would take longer than for the Surry-type of configuration.

With the compounding failure of loss-of-offsite power the condenser cooling pumps would shut down and steam flow to the condenser would be stopped to prevent damage to the condenser. The pressure in the secondary would rise to the set point of the relief valves. Atmospheric release of radioactivity would therefore occur until the affected steam generator is isolated.

Engineering analyses have been performed for this sequence which have involved a limited partial failure analysis. Two of the variables which affect the consequences of the accident have been treated probabilistically: the fraction of clad defects in the core and the time which has elapsed since the previous shutdown. Some evaluation has also been made of the possible systematic error arising from uncertainties in the retention of iodine in the steam generator. A bounding analysis was performed in which all of the iodine 
transported through the ruptured tube was assumed to be released to the environment.

The probability density functions were defined as follows:

1. Failed fuel distribution. Based upon the PWR-GALE report (7) a piecewise constant probability density function for clad defect level was developed. The density function is tabulated in Table 3.1.

2. Time since last shutdown $(\tau)$. It was assumed that events leading to iodine spiking occur with frequency $\lambda_{S}=10^{-3} \mathrm{hr}^{-1}$. The density function for $\tau$ is $\lambda_{S} e^{-\lambda} s^{\tau}$.

Because of the simple nature of these density functions, it was possible to develop a closed form expression for the density function for radionuclide release which could be solved numberically without resorting to Monte Carlo sampling. The mathematical analys is is described in detail in Appendix G. The steps taken in the analysis follow:

1. The concentration of radionuclides in the prinary system is assumed to be directly proportional to the clad defect level. For the radionuclides analyzed, the constants of proportionality were obtained from the GALE report. (7)

2. The primary concentration of iodine is modified by the factor $Z$ which is a function of time since the last shutdown. The functional form of $Z$ is described in Appendix $F$ according to spiking model $B$.

3. The flow rate from the primary system to the secondary system is described in Figure 3.8 which was developed from data in RESAR-3S. (8) The concentration of radioactivity in the primary system is assumed constant over the 30 minutes during which flow occurs.

4. The radionuclide concentration in the secondary system is well inixed. 


\section{TABLE 3.1}

FAILED FUEL PROBABILITY DENSITY FUNCTION

For $0 \leq f_{c d}<.035, g_{c d}=11.06$
For $.035 \leq f_{c d}<.065, g_{c d}=4.08$
For .065
For .125 $\leq f_{c d}<.125, g_{c d}=3.4$
For .245 $f_{c d}<.245, g_{c d}=.875$
For .515 $\leq f_{c d}<.515, g_{c d}=.344$
cd $<.755, g_{c d}=.275$

$f_{c d}=$ failed fuel fraction

$g_{c d}=$ probability density function for $f_{c d}$

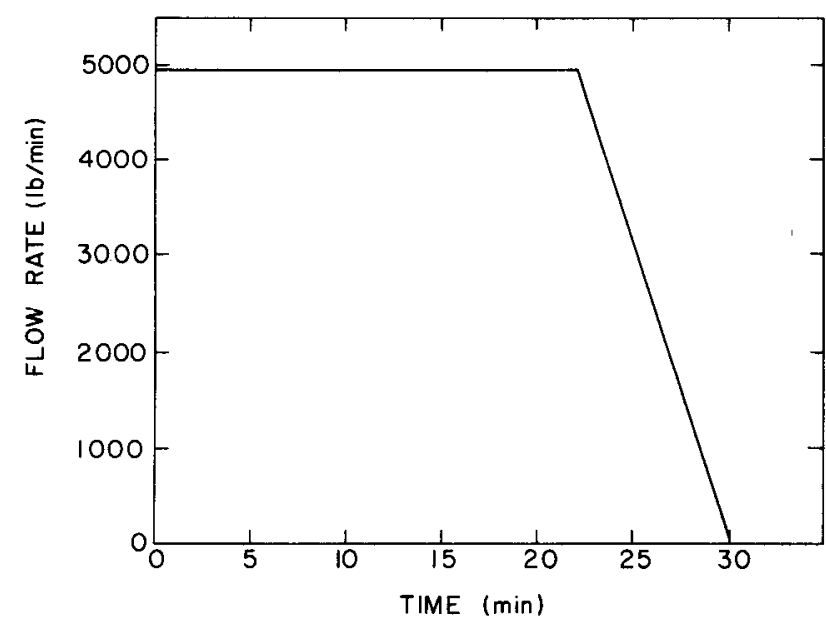

Figure 3.8. Flowrate from primary to secondary system versus time after the SGTR 
5. The mass flow rate from the faulty steam generator is assumed constant at $42,600 \mathrm{~kg} / \mathrm{hr}$ over the duration of release.

6. The equations for the concentration of radionuclides in the secondary and the release to the environment can be integrated to obtain

$$
A_{R}=K f_{C d} Z \text {, }
$$

where

$$
\begin{aligned}
A_{R} & =\text { total radioactivity released } \\
f_{C d} & =\text { fraction of clad defect } \\
Z & =\text { spiking correction. }
\end{aligned}
$$

The constant $K$ is defined in Appendix F. A partition factor of 0.1 was assumed for iodine.

7. Knowing the density functions for $f_{c d}$ and $Z$, the density function for $A_{R}$ is

$$
g_{R}(A)=\frac{1}{k} \int g_{Z}(Z) g_{C d}\left(\frac{A}{k Z}\right) \frac{1}{Z} d Z \text {. }
$$

This integral can be evaluated numerically. The complementary cumulative function can be further obtained by numerical integration of the density function. The calculation of the density function for the noble gases is somewhat simpler because the release is not affected by the spiking phenamenon. In this case Equation ( 3 ) reduces to

$$
g_{R}(A)=\frac{1}{K} \operatorname{gcd}\left(\frac{A}{K}\right) \text {. }
$$

$\underline{\text { Results }}$

The CCFs for I-131 and Xe-133 are illustrated in Figure 3.9. Because of uncertainties relating to iodine transport and retention in the secondary, a bounding calculation was also performed in which all of the iodine flowing 
through the break was assumed to be released to the atmosphere.

In Figure 3.10 the weighted CCF for the steam generator tube rupture with loss-of-offsite power is shown with the LER data at low consequences and with class 9 accidents. Also shown is the bounding calculation. This accident does not appear to be a major contributor to the risk curve because of the expected low probability of occurrence.

\subsection{REFUELING ACCIDENTS (Class 6)}

Two types of refueling accidents are included in the 10 CFR50 classification scheme:

1. Fuel bundle drop

2. Heavy object drop onto fuel in core.

In this study we have expanded the subcategories of potential refueling accidents to include:

1. Inadvertent criticality during refueling

2. Dropping the reactor vessel head onto the open reactor vessel

3. Dropping the upper core barrel assembly into an open reactor vessel

4. Dropping a fuel assembly within containnent.

Each of these potential accidents occurs within containment but at a time when the containment is being purged through the contaiment venting system. The consequences of a number of these accidents are quite sensitive to the time required to isolate the containment. Containment isolation therefore appears on each of the event trees. 


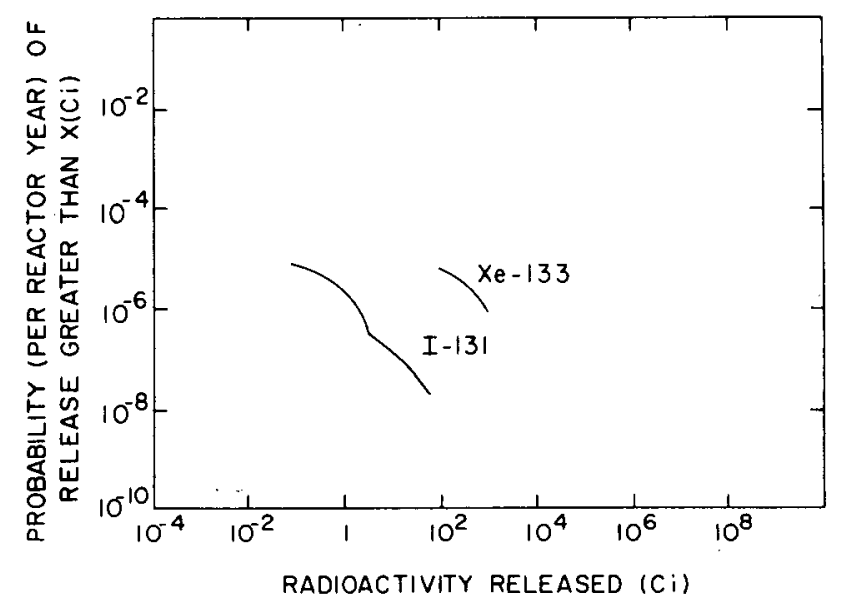

Figure 3.9. Calculated CCF for steam generator tube rupture accident

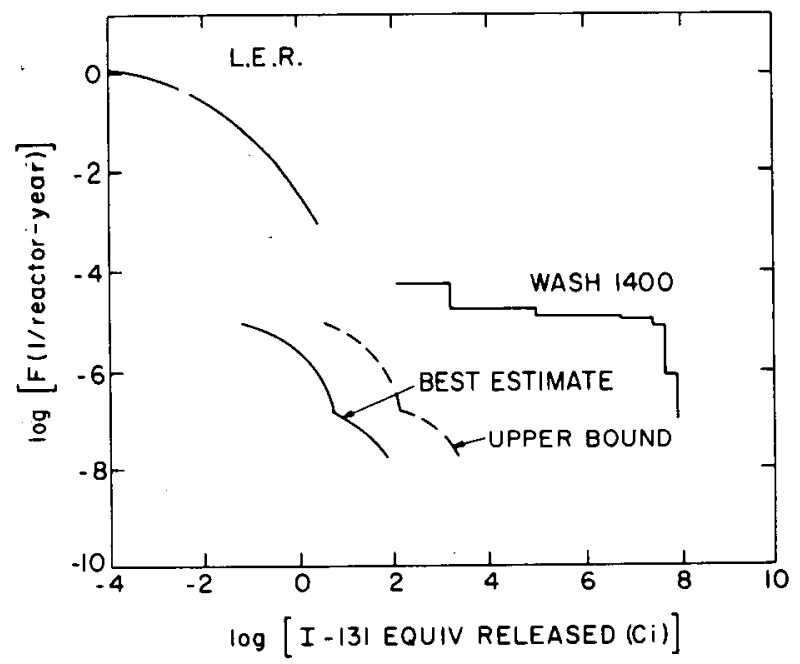

Figure 3.10. The weighted risk calculated for steam generator tube rupture compared to the LER data and the results reported in WASH- 1400 


\subsubsection{Inadvertent Criticality During Refueling}

Inadvertent criticality with the reactor vessel head of $f$ is judged to be less likely than with the head in place. During refueling the reactor cavity is filled with borated water. While the reactor vessel head is being raised from the reactor vessel, the reactor cavity is being filled with borated water, with the water level being kept just below the reactor vessel head. In order for criticality to occur, it would be necessary to dilute the boron in the water. Due to the large volume of water involved, unobserved dilution of the borated water is quite unlikely.

With the head in place but with the head studs and nuts removed, or the head nuts loosened, boron dilution could occur in the Surry plant by opening of the primary water makeup control valve. The technical specifications require that a minimum boron concentration of $2000 \mathrm{ppm}$ be maintained in any portion of the Reactor Coolant System when the reactor vessel head is unbolted, and that the boron concentration shall be checked by sampling every eight hours. At $2000 \mathrm{ppm}$ in boron concentration the reactor is approximately 10 percent subcritical. The technical specifications require that at least one source range neutron detector shall be in service at all times and at least two whenever core geometry or coolant geometry is being changed. If the boron concentration is diluted to $1500 \mathrm{ppm}$, the reactor is about 5 percent subcritical and the neutron detector count rate is doubled. It takes about one hour to dilute the boron from $2000 \mathrm{ppm}$ to $1000 \mathrm{ppm}$, at which the reactor would go critical.

Estimating the probability of inadvertent opening of a valve which causes boron dilution to be $10^{-2}$ per refueling (probably pessimistic considering 
the number of years of operating history) and estimating that this could occur with the reactor head unbolted 10 percent of the time, one obtains a probability of $10^{-3}$ that the boron dilution incident is initiated while the reactor head is unbolted. An estimate of $10^{-3}$ per incident of failing to recover either by recognizing the increase in neutron count rate or by measuring the boron concentration seems conservative. The estimate of the probability of a criticality accident is therefore $10^{-6}$ per refueling or per reactor year.

\subsubsection{Dropping the Reactor Vessel Head}

According to the analys is by Westinghouse (8), dropping of the vessel head onto the open reactor vessel does not affect fuel integrity.

\subsubsection{Dropping the Upper Core Barrel}

If the upper core barrel assembly were dropped into an open reactor vessel some fuel rod failures could occur(9). The enviromental release of radionuclides in this accident is very sensitive to whether the containment can be isolated before significant release occurs and whether the release is discharged through filter banks. In Figure 3.11 the event tree for this accident identifies pathways associated with containment isolation and filtration operation. Of course, partial isolation is also possible.

Taking an upper bound estimate of $1 / 2$ hour of crane operation for moving the upper core barrel per refueling and a probability of $3 \times 10^{-6} / \mathrm{hr}$ for crane failures $(1)$ one obtains a probability of $2 \times 10^{-6} / \mathrm{yr}$ for dropping the upper core barrel into an open reactor. It should be noted, however, that 


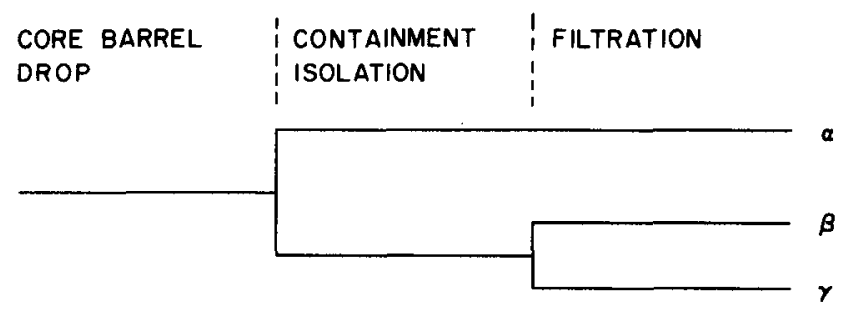

Figure 3-11. Event tree for core barre1 drop 
at the R.E. Ginna Unit I reactor, the reactor core barrel and internals were dropped 6 feet onto a storage rack as the result of failure of the crane's electromagnetic brake; the mechanical brake was not installed at the time of the occurrence which was prior to initial criticality. The incident could be an indication that $2 \times 10^{-6} \mathrm{yr}^{-1}$ is too low for this type of accident.

The relative probabilities of the different branches on the event tree are quite dependent on plant design. For example, if a push-pull-type or exhaust only sweep ventilation system is in operation over the refueling canal the released activity would be routed immediately to the purge exhaust. Although containment high radiation levels and high airborne activity levels would be detected by containment radiation monitors which would automatically stop and isolate the containment purge system, it is not clear that the containment could be isolated sufficiently rapidly to reduce the release to the atmosphere appreciably.

The exhaust air fran the containment discharges through the ventilation vent and may be bypassed through the common auxiliary building roughing, particulate, and iodine filter banks. However, it is not clear that the containment exhaust air is routinely bypassed through the auxiliary building filters during refueling. In the analys is of the core barrel drop accident only the worst sequence, in which no credit is taken for isolation or filtering, has been evaluated.

\subsubsection{Upper Core Barrel Drop Without Isolation or Filtration}

Only a scoping analys is has been undertaken for this sequence which did not include consideration of partial failures. The following assumptions have been made in the analysis. 
1. The cladding of all rods in one assembly is damaged in the accident. This assumption is not supported but is taken as a representative example. It is recognized that a greater number of 'pins could actually be damaged.

2. A radial power factor of unity is assumed.

3. The accident occurs 90 hours after shutdown which is approximately the time at which the upper core support is removed from the vessel. The quantity of radionuclides released is

$$
Q=\frac{G F}{D F} \times R F \times I \times e^{-\lambda \tau}
$$

where

$$
\begin{aligned}
\mathrm{GF} & =\text { gap fraction } \\
\mathrm{RF} & =\text { release fraction } \\
I & =\text { inventory per assembly }(\mathrm{C} i) \\
D F & =\text { decontamination factor } \\
\lambda & =\text { decay constant }(\mathrm{hr}-\mathrm{l}) \\
\tau & =\text { time after shutdown }(\mathrm{hr}) .
\end{aligned}
$$

Results

The results of Equation (3.5) are shown for the most important radionuclides in Table 3.2. In Figure 3.12 the weighted releases are shown for an accident which danages a single assembly at a frequency of $2 \times 10^{-6} \mathrm{yr}^{-1}$. The figure shows the risk attributed to only the iodine isotopes along with the total risk. Appendix $H$ provides more details on the analysis of the Upper Core Barrel Drop Accident. 
TABLE 3.2

RELEASES FOR THE UPPER CORE

\section{BARREL DROP ACCIDENT}

Radionuclide

Estimated Release ( $\mathrm{C} i$ )

$\begin{array}{lc}\mathrm{Kr}-85 & 2.3(2) \\ \mathrm{Xe}-133 & 1.6(4) \\ \mathrm{Xe}-135 & 6.1(-1) \\ \mathrm{I}-131 & 3.2(-1)\end{array}$

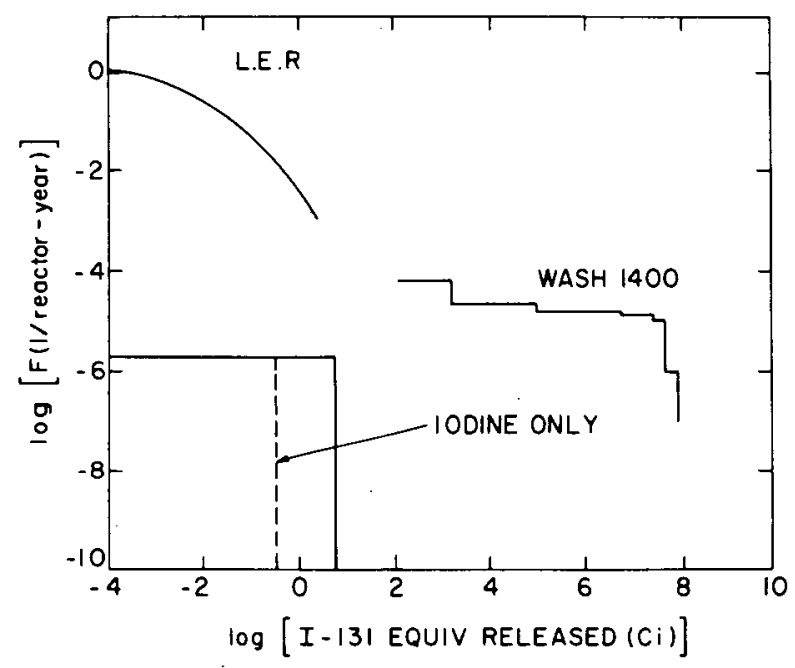

Figure 3.12. The weighted risk calculated for core barre1 drop compared to the LER data and the results reported in WASH-1400 


\subsubsection{Dropping a Fuel Assernbly}

In the NSIC file of the ORNL RECON information retrieval system 16 fuel handling errors within containment have been identified. It is not certain, however, that our list of incidents is complete. None of the incidents were noted as resulting in cladding failure. There are, on the other hand, recorded incidents $(10)$ in which fuel damage has been identified during inspection that may have resulted from handing.

On the basis that no incidents have been reported which have released gap activity as the result of a handling error but that fuel handling incidents do occur with some regularity $(.05 /$ reactor-year), a frequency of one event per 300 years of operation is assumed for an incident in which clad failure would occur. The 300 reactor years relates to the current level of operating experience.

The event tree for a fuel drop accident is identical to the one for a core barrel drop as illustrated in Figure 3.11. As for the core barrel drop accident, only the sequence with the highest assumed release has been analyzed.

\subsubsection{Fuel Assembly Drop Without Isolation or Filtration}

The probability of $3 \times 10^{-3} /$ reactor-year corresponds to the dropping of a fuel assembly which results in some degree of fuel damage. It is assumed in this analysis that when damage does occur 50 percent of the time only one row of fuel pins will be affected and that 50 percent of the time the entire assembly is damaged. Little basis exists for this assumption other than engineering judgment. 
$\underline{\text { Results }}$

The consequences of a fuel assembly drop can be inferred from the analysis for the dropped core barrel. The consequences of the failure of an entire assembly were presented in Figure 3.12. The associated frequency is $1.5 \mathrm{x}$ $10^{-3} /$ reactor-year. The consequences of failing a single row of 15 pins is merely $\frac{15}{204}$ of the values for an entire assembly. Again the associated frequency is $1.5 \times 10^{-3} /$ reactor-year.

The results of this simple analys is are plotted in Figure 3.13 in comparison with actuarial data and Class 9 accidents. This accident sequence appears to be a significant contributor to the risk curve and deserves further consideration and analysis. Also shown on this figure are the results for the core barrel drop accident which were obtained using similar assumptions. Even' though there is some uncertainty as to how many assemblies might be damaged by the core barrel, the fuel assembly drop accident appears to be controlling from a probability viewpoint.

\subsection{SPENT FUEL HANDLING ACCIDENT (ClasS 7)}

In the 10CFR50 categorization of Class 7 three sequences are considered.

1. Fuel assembly drop in fuel storage pool

2. Heavy object drop onto fuel rack

3. Fuel cask drop.

In this study an additional potential sequence is evaluated:

4. Loss of water/loss of cooling in spent fuel storage pool. 


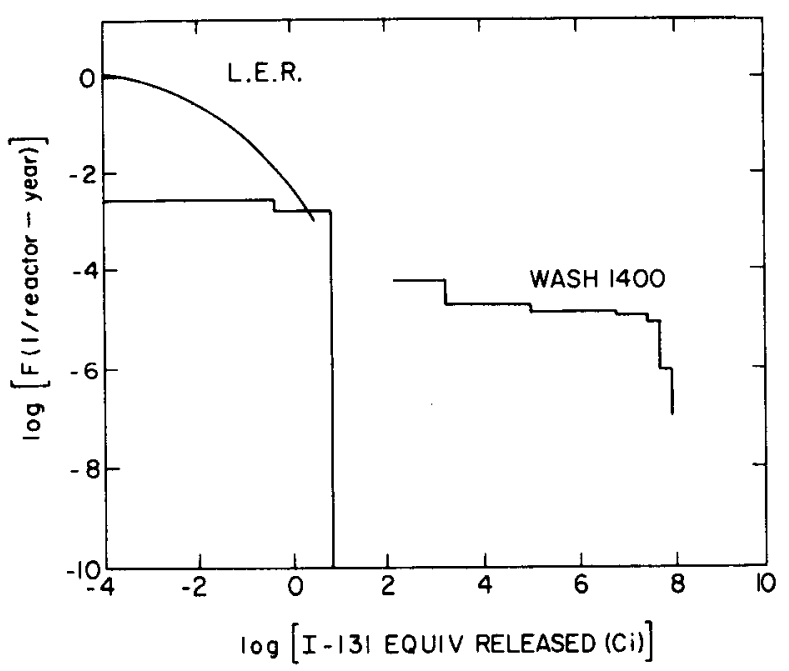

Figure 3.13. The weighted risk calculated for fuel assembly drop within containment compared to the LER data and the results reported in WASH-1400 


\subsubsection{Fuel Assembly Drop in Fuel Storage Pool}

A partial failure analysis has been performed for the case of a fuel assembly drop accident in the fuel storage pool. All fuel handling takes place under water. Thus, any gaseous fission products that are released from the fuel rod gap must bubble up through the water. During this process some iodine will be removed from the bubble and will go into solution in the storage pool water. Not all the iodine in the gap of the cladding is in gaseous form so that only a fraction will be released in the bubble.

There is some possibility that a percentage of the iodine that is dissolved in the storage pool water will be converted into methyl-iodine and other organic forms which would be released from the pool. The iodine potentially available for conversion to methyl-iodine includes all of the iodine in the gap which could go into solution, not just the iodine in vapor form. The release of iodine from the auxiliary building in this accident will depend on whether or not the auxiliary building filter is working. An event tree could therefore be drawn which identified filter failure as a subsequence of this accident. In the analysis that has been performed, however, the possibility of filter failure has been included probabilistically. The distribution function that has been calculated therefore includes both possible branches on the tree.

Many of the variables on which the release depends behave randomly. In order to perform the partial failure analysis described here for iodine release a Monte Carlo computer code, RADREL, was written. RADREL is described in more detail in Appendix I which describes the analysis of this accident. 
The release of elemental or inorganic iodine from the pool can be expressed as

$$
Q_{I}=\alpha \times N_{R} \times G I \times R F \times e^{-\lambda \tau} \times D F_{W}^{-1} \times X_{i n}
$$

where

$$
\begin{aligned}
& \alpha= 0 \text { or } 1 \text { depending on whether an incident leading to fuel has } \\
& \text { occurred } \\
& N_{R}= \text { number of rods suffering clad damage } \\
& G I= \text { gap inventory }(\mathrm{C} i) \\
& R F= \text { release fraction } \\
& \lambda= \text { decay constant } \\
& \tau= \text { time of drop after shutdown } \\
& D F_{W}= \text { decontamination factor of water } \\
& X_{i n}= \begin{array}{r}
1 \text { if auxiliary filter fails } \\
\left(D F_{A}\right)-1 \text { if auxiliary filter functions }
\end{array} \\
& D F_{A}= \text { decontamination factor of auxiliary building filter. } \\
& \text { Density functions are assumed for the following variables: }
\end{aligned}
$$

1. $\alpha$ - The conditional probability of at least one pin failing $(\alpha=1)$ in a drop accident is $P_{\alpha}$. In Appendix I the probability density function for $P_{\alpha 1}$ is developed based upon six drop accidents in which no failures were observed.

$$
p\left(p_{\alpha 1}\right)=7\left(1-p_{\alpha 1}\right)^{6}
$$

The expection value of $p_{\alpha 1}$ is 0.125 which was used in the base case. 2. $N_{R}$ - The conditional probability distribution for $N_{R}$ is

$$
\begin{aligned}
& \operatorname{pr}\left\{N_{R}=15 \mid \alpha=1\right\}=p_{1} \\
& \operatorname{pr}\left\{N_{R}=204 \mid \alpha=1\right\}=1-p_{1}
\end{aligned}
$$


where $p_{1}$ is uniformly distributed between 0.2 and 0.8 . For the base case $p_{1}=0.5$. There is little basis for the assumed distribution other than engineering judgment.

3. Gap inventory, GI. Two power levels per assembly were considered, 20 Mwt and 15 Mwt. The higher power assemblies are moved prior to lower power assemblies, so a time-dependent relationship was used for the probability of picking a high power assembly, $\mathrm{P}_{\mathrm{H}}$.

$$
\mathrm{P}_{H I}=1.1-0.004 \mathrm{t}
$$

where $t$ is a random variable unifomly distributed between 100 and 200 hours. The probability of picking a low power assernbly is $1-\mathrm{PHI}_{\mathrm{I}}$. Thus at $t=100 \mathrm{hr}$ it is assumed that the assembly which has been mishandled has power 20 Mwt with probability 0.7 , while if the accident occurs when $t=200 \mathrm{hr}$, the assembly power is 20 Mwt with probability 0.3 .

4. Accident tine, $\tau$. The factor $e^{-\lambda \tau}$ accounts for decay of the radionuclide from the time of shutdown $(t=0)$ until the time $t$ of the accident. The randon variable $\tau$ was taken to be unifomly distributed between $100 \mathrm{hr}$ and $200 \mathrm{hr}(11)$.

5. Decontamination factor for pool water, $D F_{W}$.

$$
D F_{W}=73 \exp \left\{0.313 t_{B} / D\right\}
$$

where

$$
\begin{aligned}
t_{B} & =\text { rise time of bubble }(\mathrm{sec}) \\
D & =\text { effective bubble diameter }(\mathrm{cm}) .
\end{aligned}
$$

The effective bubble diameter was treated as a random variable with a uniform distribution between $0.5 \mathrm{~cm}$ and $2.0 \mathrm{~cm}$. This is slightly conservative based on data in Reference 12. The bubble rise time was 
related to the height below the surface of the water at which the failure occurred by introducing a bubble rise velocity. The velocity was taken as $149 \mathrm{~cm} / \mathrm{sec}(12)$ corresponding to the release pressure of1200 psi in the fuel rod gap at the end of life. The height below the surface of the water at which the accident occurs was taken as either 26 feet or 40 feet, depending on whether the accident occurred at the top of the fuel bundle storage array or at the bottom of the pool. The probability of occurrence at the 26-foot level or 40-foot level was assigned the probability $\mathrm{p}_{H}$ and $\left(1-\mathrm{p}_{H}\right)$ respectively. $\mathrm{P}_{\mathrm{H}}$ was taken as uniformly distributed between 0.25 and 0.75 . For the base case $\mathrm{p}_{H}=0.5$.

6. $X_{i n}$ - This factor accounts for the possibility of failure or bypass of the filters in the auxiliary building. If the filter works $x_{i n}=$ $D F_{A}^{-1}$. If filter fails $X_{i n}=1$. The probability of failure $\operatorname{pr}\left\{x_{i n}=1\right\}=0.01$ based on discussions with NRC staff. The value of $\mathrm{DF}_{\mathrm{A}}$ was chosen as 100 based on an assessment by $\mathrm{BCL}$.

The release of organic iodine can be written as

$$
\text { Qorg }=\alpha N_{R} G I e^{-\lambda \tau} F_{\text {org }} X_{\text {org }}
$$

where

$F_{\text {org }}=$ fraction of iodine converted to organic form in pool water. $x_{\text {org }}=1$ auxiliary filter fails $\left(D F_{\text {Aorg }}\right)^{-1}$ auxiliary filter functions

$D F_{\text {Aorg }}=$ filter decontamination factor for organic iodine. Equations (3.6) and (3.11) differ in that the $D F_{W}$ is set to unity for organic iodine. Forg was taken as 1 percent; it is considered to be highly 
uncertain. Data from experiments or incidents in which iodine has been released at very low concentration in pools have indicated relatively high conversion to organic iodine (as high as 10 percent). A mechanism by which this extent of conversion could take place in aqueous solution is not known, other than by radiolysis. The level of radiation in the pool is not nearly as high in the pool as in a reactor, however. It is assumed that all of the iodine in the gap of a failed fuel pin is available to be dissolved and potentially converted to organic iodine.

\section{Results}

The best estimate of the complementary cumulative distribution function was obtained by performing a Monte Carlo analys is of the releases described by Equations (6) and (11) with $\alpha=1$. The results are then multiplied by the probability per reactor-year that an accidental drop will occur and by the probability that, if a fuel handling accident has occurred, at least one rod fails. Base case values were used for the parameterized density functions for $N_{R}, G I, H$, and $X_{i n}$.

Each Monte Carlo run was divided into 30 experiments consisting of 1000 histories (trials). The results of each trial were then grouped into a number of bins according to the quantity of iodine released. The complementary cumulative distribution was obtained by summing bins. By dividing the total number of trials into 30 separate experiments a statistical variation in the distribution function could be determined. The RADREL code which was used to perform the Monte Carlo analys is is described in more detail in Appendix I. 
In order to develop an understanding of the magnitude of the uncertainty band about the CCF which arises from ignorance as to the true magnitude or variation in variables, the parameters describing the density functions for $N_{R}, G I, a, H$, and $X_{i n}$ themselves treated as variables. Five hundred RADREL runs using random values of the parameters were performed. The five hundred values of the CCDF corresponding to a particular release were then ordered. From this 50 percent confidence limits were found.

The CCFs for inorganic and organic iodine releases are illustrated in Figures 3.14 and 3.15 with the associated 50 percent conf idence bounds. Figure 3.16 shows the CCF for weighted releases of organic, inorganic and noble gases for the fuel handling accident in comparison with the Class 9 accidents and actuarial data.

\subsubsection{Heavy Object Drop Onto Fuel Rack}

The likelihood of dropping a fuel assembly onto the fuel rack appears to be much higher than for any other heavy object which could cause fuel damage because of physical restraints and administrative controls in the use of cranes in this area. The fuel assembly drop accident was considered in the previous section.

\subsubsection{Fuel Cask Drop}

In the Surry facility a fuel cask cannot be dropped greater than 30 feet. In the licensing of spent fuel shipping casks, analyses and model experiments are performed which show that the cask will survive a 30-foot drop intact. 


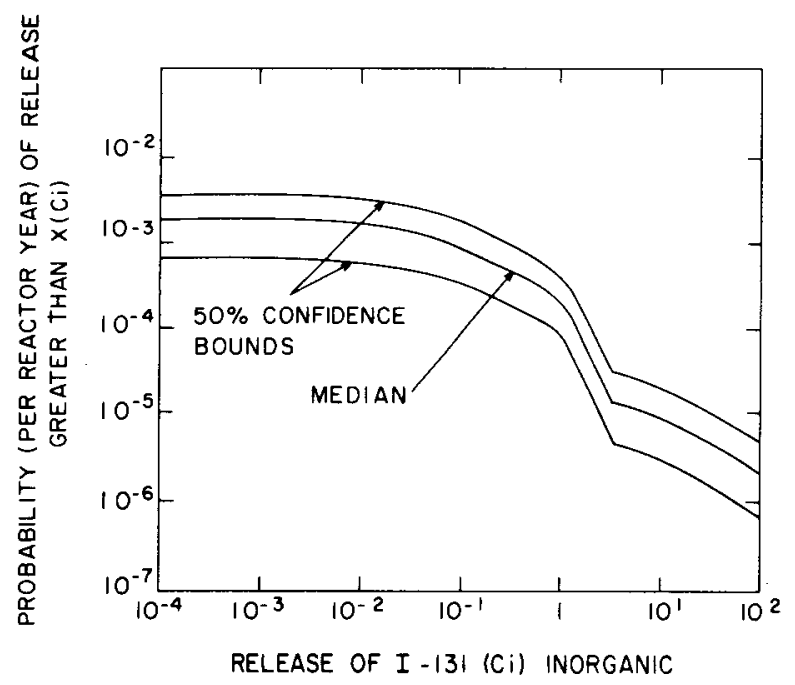

Figure 3.14. CCF for fuel assembly drop in fuel storage pool: lower and upper limits of the $50 \%$ confidence interval and the median values of the probability (per reactor-year) of exceeding $X$ curies of I-131 (inorganic) based on 500 RADREL simulations

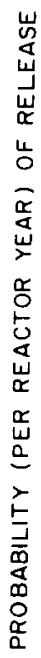

Figure 3.15. CCF for fuel assembly drop in fuel storage pool: lower and upper limits of the $50 \%$ confidence interva 1 and the median values of the probability (per reactor-year) of exceeding $X$ curies of I-131 (organic) based on 500 RADREL simulations

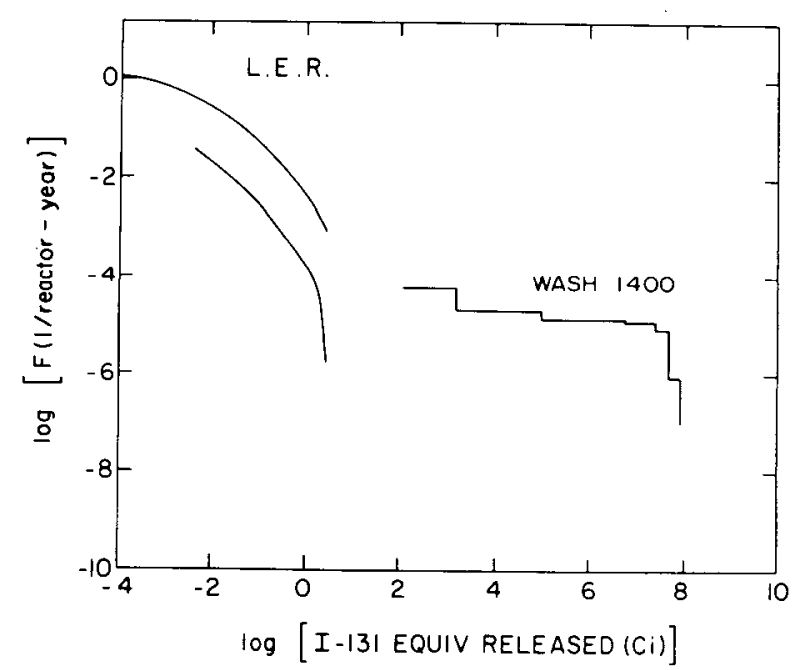

Figure 3.16. The weighted risk calculated for fuel assembly drop in spent fuel storage pool compared to the LER data and the results reported in WASH- 1400 
Shipping casks are not moved over fuel in the fuel storage rack in the spent fuel storage pool. The likelihood of release in a cask drop accident is therefore small.

\subsubsection{Loss of Water/Loss of Cooling in Spent Fuel Storage Pool}

The most serious failure in the spent fuel pit system would be the complete loss of water. This type of failure would result in the loss of cooling and removal of radiation shielding of the spent fuel and could result in the subsequent release of gaseous airborne radioactivity to the environment. The water in the spent fuel pit could be lost by the water being pumped, siphoned, leaked, or vaporized out of the pit. However, the water would most likely be lost due to a combination of these factors. The fuel building itself has only a limited capability to contain the water and vapor lost from the pit. Therefore, radioactivity would also be transferred to the external environment through the loss of water and steam escaping from the fuel building following the loss of cooling incident. The arnount of radioactivity released will increase as a function of the rise in the temperature of the spent fuel assemblies, the amount of water and steam and other gases that escape the fuel building, and the length of time that these assemblies are exposed to the environment.

The probability of a complete loss of water from the spent fuel pit by failures involving the pit cooling system is estimated to be orders of magnitude below accidents initiated by external events such as earthquakes. Ample sources of make-up water are available to mitigate this type of accident. 
The more likely accident sequences that could result in release of radioactivity to the environment involve loss of spent fuel pool cooling, subsequent heat-up of the spent fuel pit water, and the pool boiling which will result in the release of radioactivity. The probability versus release magnitude are estimated in this analysis for accident sequences involving loss of spent fuel pit cooling.

Prior to the transfer of spent fuel fram the reactor to the spent fuel storage pool, water will enter the cladding of those pins which have experienced failure in operation. If, during the heatup of the pool, boiling occurs in the gap of these failed pins, it is likely that a release of radioiodine will occur to the pool water analogous to the spike that occurs at the time of reactor startup after a short shutdown period. The mechanisms of spiking are not adequately understood to accurately predict the size of the spike that would occur. General Electric(13), estimates that startup spikes are typically half as large as decampression spikes. This appears to be consistent with PWR behavior in which the inventory of iodine available for release is noticeably depleted by the occurrence of a spike such that subsequent spiking conditions within the period of 1 to 2 days lead to smaller spikes (14).

The quantity of radioiodine release in a spiking sequence in an operating reactor can be estimated from data tabulated by Pasedag(15) as a function of failed fuel fraction.

The quantity of inorganic iodine released from the pool, assuming $1 / 3$ of a core is affected, is then

$$
Q=W_{B} \times \frac{S R}{6 M} \times e^{-\lambda t_{1}} \times F C \times D F_{W} \times D F_{A} \times t_{R}
$$


where

$W_{B}=$ steam mass generation rate from boiling pool

$S R=$ spike release of $I-131$ per percent clad defected in operating reactor

$M=$ mass of water in pool

$\lambda=$ decay constant

$t_{1}=$ time since last shutdown

$t_{R}=$ repair time

$F C=$ percentage of clad defects

$D F_{W}=$ decontamination factor for pool

$D F_{A}=$ decontamination factor for filter.

A fraction of the iodine released to the pool will be in the form of volatile organic iodine or will be converted to organic iodine. In experiments and incidents in which iodine has been released to pools, the fraction of organic iodine has varied inversely with concentration. At very low concentrations such as in the storage pool, the percentage of organic iodine could be as high as 10 percent. Organic iodine was not included in the partial failure analysis, however, because the results for inorganic iodine indicated that this accident is not a major contributor to the risk of class 3-8 accidents.

The analys is methodology was designed to account for the large number of dependencies among the factors affecting the probability versus release magnitude estimate. Most of these factors are, however, dependent upon the time after refueling at which the accident (loss of pit cooling) occurs. For instance, the time after refueling $\left(t_{1}\right)$ at which the accident occurs affects: 
- The radioactive inventory of the fuel rods

- The heat load to the pool, which in turn affects the time required to raise the fuel pit to boiling $\left(t_{2}\right)$, and the boil-off rate.

- By virtue of $t_{2}$ above, the probability of the pit reaching boiling

- By virtue of the boil-off rate, the rate at which $1-131$ is released to the atmosphere.

Therefore, the analys is methodology was structured around the dependencies inherent in the time after refueling at which time the accident occurs. If it is assumed that the accident occurs on a given day after refueling, the relationships between the various factors affecting probability versus release magnitude can be defined and incorporated into the methodology. This suggests a partition of the sample space by day after last refueling, which was the approach taken. The probabilities versus release magnitude are estimated given that the accident occurs on each of the specified days after refueling, and the results are sumned using the law of the total probability to obtain the probability versus release magnitude for the accident sequence.

$$
\begin{aligned}
& A_{i}=\text { the event that cooling is lost on the } i^{\text {th }} \text { day after re- } \\
& \text { fueling, and the accident results in pit boiling } \\
& R_{j}=\text { a specified release magnitude of size } R_{j} \cdot
\end{aligned}
$$

By the law of total probability, the probability of the specified release magnitude greater than $R_{j}$, from this accident sequence is

$$
P\left[R_{j}\right]=\sum_{i=k}^{n} P\left[R_{j} / A_{i}\right] P\left[A_{i}\right]
$$


where

$$
\sum_{i=k}^{n} \begin{aligned}
& \text { refers to the summation over the } n \text { days between refuelings, } \\
& \text { starting with the } k^{\text {th }} \text { day }(k=5 \text { for the analys is reported }
\end{aligned}
$$

For the $i^{\text {th }}$ day after refueling, the model estimates the conditional probability of release greater than $R_{j}$ given that loss of cooling occurs on the $i$ th day $\left(P\left[R_{j} / A_{j}\right]\right)$, and the marginal probability that loss of cooling occurs on the $i$ th day $\left(P\left[A_{j}\right]\right)$. These two probabilities are estimated by different techniques. The conditional probability is estimated by a partial failure analysis from a consideration of the factors that define the state of the systen at the time of the accident, e.g., failed fuel percent, time required to repair the cooling system or mitigate the accident, etc. The marginal probability is estimated from a fault tree analys is of the fuel pit cooling system as described in Appendix $J$.

Both the marginal and conditional probability estimates are uncertain by virtue of the systematic error in the parameters upon which these estimates depend. The marginal probability estimate, $P\left[A_{j}\right]$, is uncertain due to the uncertainty in the failure rates of component failures leading to the event "the fuel pit begins to boil." The conditional probability, $P\left[R_{j} A_{j}\right]$, is uncertain due to uncertainty in several physics and engineering parameters, namely the iodine partition factor, and filter efficiencies in the hot, humid conditions that would result from a boiling spent fuel pit. Thus, both the marginal and conditional probabilities are more correctly thought of as distributions of possible probabilities for the accident sequence. These distributions reflect the best estimates of the systematic error in the parameters that are used to estimate the probabilities. 
The probability of a specified release maynitude greater than $R_{j}$, $P\left[R_{j}\right]$, is thus also a distribution, since $P\left[R_{j}\right]$ is the sum of products of the conditional and inarginal probabilities, both of which are distributions. To estimate the distribution of $P\left[R_{j}\right]$, the model propagates the distributions of $P\left[R_{j} / A_{j}\right]$, and $P\left[A_{j}\right]$ to $P\left[R_{j}\right]$, using Equation 3.13. The model performs this error propagation for several specified values of $R_{j}$. The medians of the distributions for $P\left[R_{j}\right]$ define the centrality and shape of the probability versus release magnitude curve for the boiling fuel pit accident. The five and ninety-five percent confidence levels of the distributions for $P\left[R_{j}\right]$ serve to bound the probability versus release magnitude. The end product of the modeling effort is a complementary cumulative curve for probability versus release magnitude, and confidence bounds on the curve.

In Table 3.3 the sources of variation and their treatment in the partial failure analysis are described. In Table 3.4 the sources of uncertainty in the release magnitudes that result from ignorance are identified and their treatment is described.

The bounds of the probability of a release greater than $R_{j}$ of a given size are obtained by estimating the distribution of the probability of release greater than $R_{j}$. The distribution of $P\left[R_{j}\right]$ is obtained by a straightforward error propagation using the SAMPLE code. From Equation 3.13 the distributions of $P\left[R_{j} / A_{j}\right]$ and $P\left[A_{i}\right]$ are propagated to obtain the distribution of $P\left[R_{j}\right]$.

\section{Results}

The model was evaluated for the Surry spent fuel pit for the case where the pit was filled with three full cores, the results of nine refuelings, 
TABLE 3.3

SOURCES OF VARIATION THAT

INFLUENCE RELEASE MAGNITUDE

Source of Variation

1. Time after refueling

2. Previously stored core sections

3. Initial pool temperature

4. Failed fuel percent

5. I-131 spike given failed fuel percent

6. Cooling system repair time

\section{Affects}

Pool heatup rate; I-131 spike

Pool heatup rate

Time to boil

I-131 spike

I-131 in water after spike

Amount I-131 released; probability of a boiling pit

\section{Assumed \\ Statistical Characterization}

Uniform distribution between refuelings

Deterministic

Normal distribution between 70 and $120 \mathrm{~F}$

Lognomal distribution between $.12 \%$ and $1 \%$

Empirical distribution from reactor spikes, modified for spent fuel pool accident

Empirical distribution from WASH 1400 , Appendix III

TABLE 3.4

SOURCES OF IGNORANCE THAT INFLUENCE ESTIMATION

OF PROBABILITY VERSUS RELEASE MAGNITUDE

\section{Source of Ignorance}

1. 1-131 partition factor

2. Filter efficiency

\section{Affects}

I-131 releases rate

I-131 released to atmosphere
Assumed

Statistical Characterization

Lognormal distribution between .0016 and .16

For elemental I-131, lognomal distribution between 99 and 99.99 percent. For organic I-13I, lognomal distribution between 10 and 90 percent. 
conducted a half year apart. The risk per reactor year was evaluated for the period after the ninth refueling.

Figure 3.17 shows the complementary cumulative curve for the release of this accident in the context of actuarial data and the WASH-1400 results for meltdown accidents.

Two aspects of the probability versus release magnitude for this accident sequence are apparent:

- The release magnitudes and probabilities for the accident are both low compared to other class $3-8$ accidents.

- The error bounds on the estimated probability of stated release magnitudes are large - greater than a factor of 10.

The bounds on estimated probabilities are large due primarily to the uncertainty with which the partition factor is known. However, even the upper 95 percent bound on the probability versus release magnitude curve indicates a relatively low probability for moderate releases. Appendix $J$ provides a description of the analysis carried out for this accident.

\subsection{ACCIDENT INITIATING EVENTS CONSIDERED IN DESIGN BASIS EVALUATION IN THE SAFETY ANALYSIS REPORT (CTASS 8)}

The design basis accidents, particularly the loss-of-coolant accident, have in the past received more attention from a regulatory and safety research viewpoint than other accidents. However, these accidents do not necessarily involve the greatest releases of radionuclides nor do they necessarily represent a significant contribution to risk. In the 10CFR50 classification scheme, three Class 8 accidents are identified for PWR's. 
1. Loss-of-coolant accidents

2. Rod ejection accident

- 3. Steamline break outside contaiment.

In the consideration of steamline breaks in this study, the possibility of a break within containnent is also evaluated as well as the potential for a steam generator tube to rupture as a result of loads imposed by a steamline break.

\subsubsection{Loss-of-Coolant Accidents}

Loss-of-coolant accidents were considered explicitly in WASH-1400. (1) PWR release categories 8 and 9 correspond to large pipe-break accidents in which the containment fails to isolate and isolates respectively. The release estimates in WASH-1400(1) are very conservative, however. It was assumed in those calculations that the WASH-1400(1) gap inventory would be released from all fuel rods in the accident. Not only do realistic analyses of fuel cladding temperatures indicate that only the highest power rods will fail, experiments at ORNL (16) have shown that the release of iodine in this type of temperature transient is approximately two orders of magnitude lower than the WASH-1400(1) release.

Because the containment is so effective in containing radioactivity releases from the primary system, ways in which the containment could be bypassed in a LOCA have been evaluated. The types of faults investigated were sampling and test valves left open following maintenance, valve and purnp leaks, surnp pump failures, and the reverse flow of radioactive fluids into vented storage containers. Since the probability of a large LOCA is $10^{-4} /$ reactor year according to WASH-1400, the likelihood of any of these 
compounding faults would have to be near unity to represent a significant contributor to class 3 to 8 accident risk. The system evaluation performed for these potential release pathways is presented in Appendix $K$. None of the pathways appeared to be a significant contributor to risk.

\subsubsection{Rod Ejection Accident}

The rod ejection accident is similar in nature to a loss-of-coolant accident since it involves a break in the primary system in addition to a power transient. The probability of a rod ejection accident is expected to be quite small and was thus defined out of the program's scope. As a result, this accident has not been investigated in this proyram.

\subsubsection{Steamline Break}

The location of a steamline break in Surry can have a major influence on the probabilities and consequences of the accident. Three possible locations were considered for the break:

1. Inside of containment - Zone 1

2. Outside of containment and upstream of the isolation valves Zone 2

3. Downstreain of the isolation valves and upstream of the turbine Zone 3.

These locations are indicated in Figure 3.18. Utilizing the Surry FSAR, approximate total pipe lengths for each zone have been developed. The probability of having a large bore pipe rupture in a nuclear plant is discussed in 


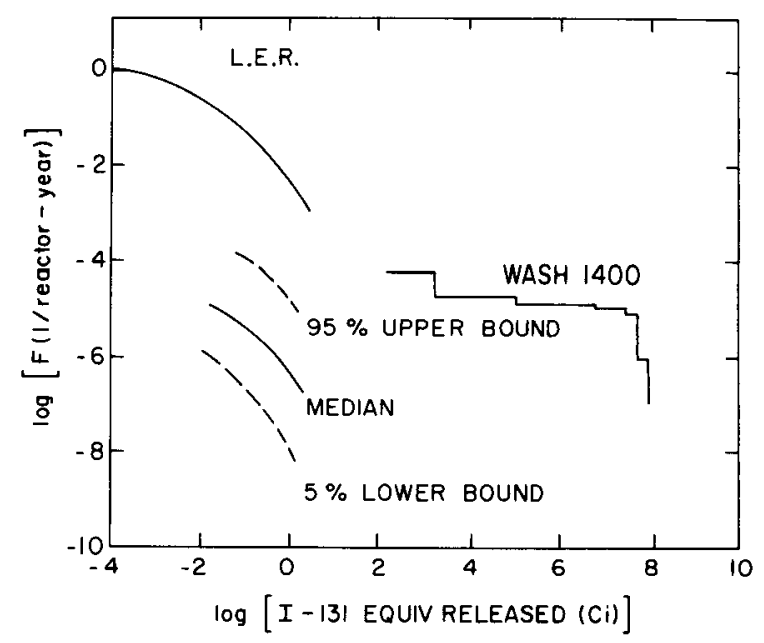

Figure 3.17. The weighted risk calculated for loss of cooling in the spent fuel pool compared to the LER data and the results reported in WASH-1400

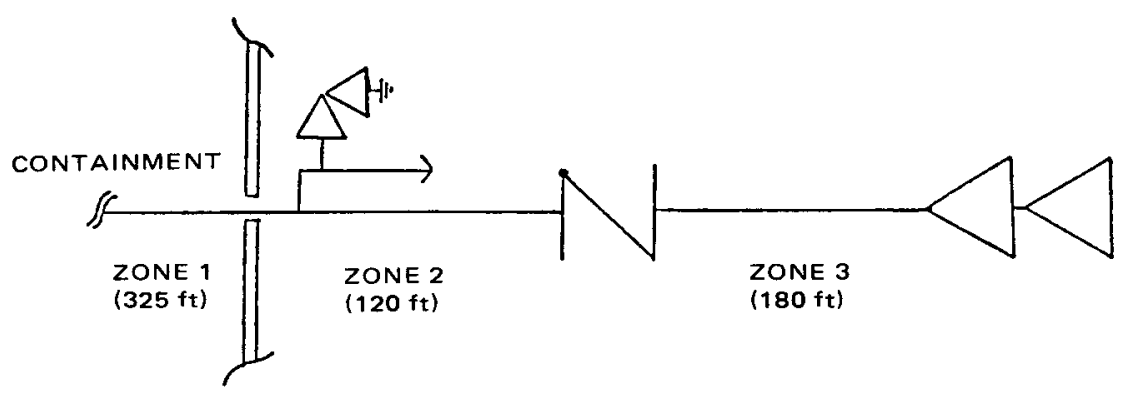

Figure 3.18. Simplified schematic of main steam line 
Appendix L. It is concluded that, at this time, there exist major discrepancies in current literature regarding pipe rupture. In this report a range of steamline break probabilities is taken as

$$
10^{-2} / \mathrm{yr} \leq \mathrm{P}(\mathrm{SLB}) \leq 10^{-4} / \mathrm{yr} \text {. }
$$

It is recognized, however, that the probability could be lower.

The forces imposed on stearn generator tubes in a steamline break could lead to tube failure if the tubes are sufficiently degraded. In Appendix M the conditional probability was estimated

$$
P(S G T R / S L B)=5 \times 10^{-2}
$$

The uncertainty in this probability is quite large.

$\underline{\text { Zone } 1}$

The steamline break within containment requires an additional failure, that of breach of contaiment, to release radionuclides to the biosphere. Two mechanisms have been evaluated that could lead to such a break. The first involves failure to isolate main feedwater with the potential to overpressurize containment. A fault tree was developed for the failure to isolate main feedwater which resulted in a probability of $10^{-4}$. The combined probability of the steamline break with failure to isolate main feedwater is therefore quite small. In addition, the accident would probably not result in containment failure.

The other potential breach mechanism would be a leak in the ECC system in the recirculation mode following a compounding stean generator tube rupture. The combined probability for these events is too small to affect the risk curve. 
Zone 2

The second zone is of most concern because a pipe rupture in this region will result in a release outside of containment which, in general, cannot be isolated by the closure of valves. It should be noted that the surry plant has isolation valves in the primary loops that can bring this sequence to a conclusion comparatively rapidly. The analys is presented here does not take credit for this isolation capability because it is not typical of PWR's. In Figure 3.19, an event tree for a steamline break is shown which shows the potential for compounding by a steam generator tube rupture. If a tube rupture does not occur, the consequences of the accident are limited by the radionuclide inventory of the secondary system. The consequences in the event of the tube rupture could be much greater. For this reason this branch of the tree has been analyzed in this study.

\section{Zone 3}

The last section of the steamline break analysis will not be discussed in detail since the occurrence probabilities and anticipated releases for these sequences are low. In order to obtain releases comparable to those in the second zone, it would be necessary to have main steam isolation valve failure in addition to a steam generator tube rupture.

\subsubsection{Steanline Break With Steam Generator Tube Rupture}

The assumed sequence of events in this accident is as follows:

1. A steamline fails in the region outside containment where it cannot be isolated. 
2. The affected steam generator blows down to the atmosphere in a short period of time.

3. A tube rupture in the affected steam generator.

4. The reactor trips.

5. The ECCS is activated.

6. The primary system is cooled down and depressurizes.

7. Feedwater flow to the affected steam generator is stopped.

A partial failure analysis was not performed for this accident sequence. The quantity of a radionuclide released in the accident is given by

$$
Q=A P W_{T} S / D F
$$

where

$Q=$ quantity released

$A P=$ primary coolant inventory

$W_{T}=$ total amount of primary fluid vented to the environment

$D F=$ decontamination factor in the affected steam generator

$S=$ spiking multiples to account for iodine buildup in the accident

\section{Results}

In calculating the consequences of this accident, average or expected values of the variables affecting the release were assumed as follows:

1. Primary System Fluid Vented (WT). The modified Burnell critical flow model (17) was used to estimate the amount of primary system fluid vented to the secondary using a primary system pressure and temperature time history modified from results presented by Fontecilla and Grimes(18) with the secondary system at atmosphere pressure. 
2. Decontamination Factor (DF). The assumption is made that none of the primary system fluid is retained in the secondary but rather is all vented to the environment. This implies that the decontamination factor for this venting process is unity for the iodine as well as the noble gases.

3. Radionuclide Inventory (AP). The assumed inventory of radionuclides in the primary coolant is that given in Table 1 from the PWR GALE report $(7)$.

4. Spiking Multiplier (S) (Iodine Isotopes Only). Iodine spiking can contribute to an accident sequence either as the result or a prior shutdown or due to spiking during the accident itself. Since an accident would have to occur within a few days of a spike for the iodine concentration to be elevated, the probability of occurring within such a time window is relatively small. We have only considered the contribution from the accident itself in this analysis. Based on a spiking time of 6 hours and the average of the distribution for spike magnitude in Appendix F, a spiking multiplier of 3.3 was obtained.

The predicted releases of important radionuclides are presented in Table 3.5. The complementary cumulative distribution functions for weighted releases are illustrated in Figure 3.20. The assumed probability of the accident as shown in the plot is

$$
\begin{aligned}
P & =P_{S L B} \quad P(\text { SGTR/SLB }) \\
& =\left(10^{-3}\right)\left(5 \times 10^{-2}\right) \\
& =5 \times 10^{-5}
\end{aligned}
$$

An increase in the probability by two orders of magnitude would still not impact the total risk of Classes 3 to 8 accidents significantly. 


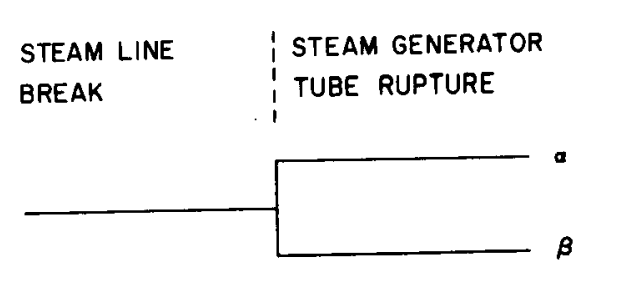

Figure 3.19. Event tree for steam line break accident

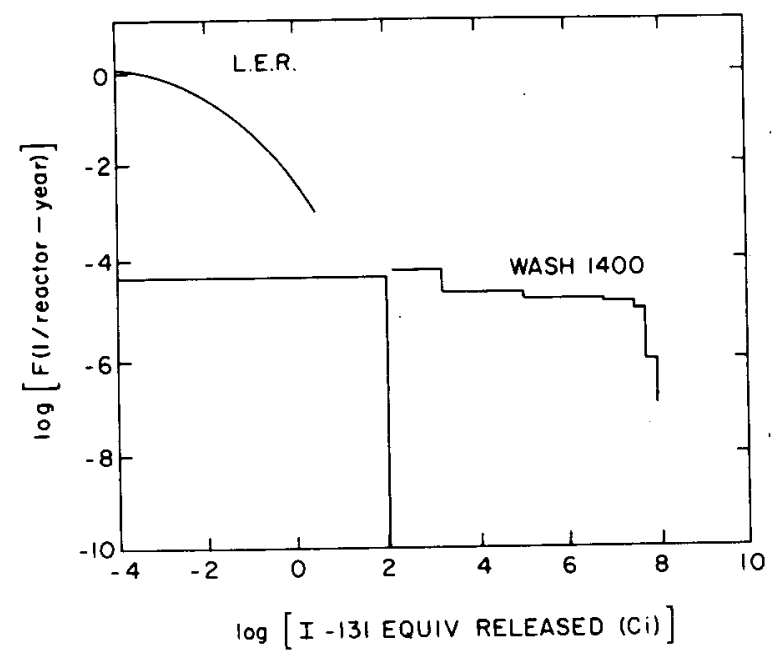

Figure 3.20. The weighted risk calculated for steam line break compared to the LER data and the results reported in WASH-1400

TABLE 3.5

RELEASES FOR STEAMLINE BREAK WITH

STEAM GENERATOR TUBE RUPTURE

Radionuclide

$\mathrm{Xe}-133$

I-131

I -132

I -133

I -134

I -135
Estimated Release (Ci)

1800

89

33

125

16

63 
3.6 ESTIMATION OF FREQUENCY OF VARIOUS EVENT SEQUENCES IN LOSS OF FEEDWATER TLOF) TRANSIENT

Recent events have shown tht a loss of feedwater, together with several compounding failures, may have serious consequences. The loss of feedwater transient is a routine operational occurrence, on average about three times a year, and usually does not lead to any untoward consequences. The transient is not analyzed in detail in this study. The work presented here is a first cut probabilistic assessment of various event sequences in a loss of feedwater transient for a Pressurized Water Reactor of Westinghouse design. The frequency of various end events are evaluated as point estimates with no error propagation.

\subsubsection{Event Trees for the Loss of Feedwater Transient}

A detailed LOF event tree, pruned and scoped to include events in the primary side is shown in Figure 3.21. The following symbols are used in the loss of main feedwater event tree:

TM: Interruption of main feedwater system

K: Reactor Protection system does not operate

L: Auxiliary Feedwater systes does not operate

$P_{1}$ : Pressurizer relief valve does not open on transient demand

$Q_{1}$ : $\quad$ Pressurizer relief valve does not reclose

$P_{2}$ : Pressurizer safety valve does not open on transient demand

Q2: Pressurizer safety valve does not reclose

A comparison of the LOF event tree adapted from the generic transient event tree in WASH-1400(1), shown in Figure 3.22 , and the loss of feedwater 
transient event tree in this study shown in Figure 3.21 indicates the following differences:

- In WASH-1400 the pressurizer relief valves and safety valves opening on transient derland were lumped together as a single event, designated as P. The event tree used in the present study distinguishes between the pressurizer relief valves and safety valves opening on transient demand. Thus, there are two separate events in tree, designated as $P_{1}$ and $P_{2}$. The probability of failure of the relief or the safety valve to open on transient demand is quite different. Thus, it is more realistic and therefore necessary to separate the two events.

- Given a LOF transient without scram and the auxiliary feedwater not being available, the event tree in this study does not take into account the event whether the pressurizer safety and/or relief valves open on transient demand. In contrast the WASH-1400 tree considers the above mentioned event. The inclusion of the above mentioned event is not necessary since irrespective of the outcome of this event the transient could lead to core melt.

\subsubsection{Estimation of the Probability of the Events in the Transient Initiated by a Loss of Feedwater}

The calculated probabilities of each sequence is shown in Table 3.6, and the individual frequencies are discussed as follows.

\subsubsection{Frequency of Loss of Feedwater Event (TM):}

According to WASH-1400(1) there could possibly be three main feedwater interruptions per reactor year of which two per 10 reactor-years can be 


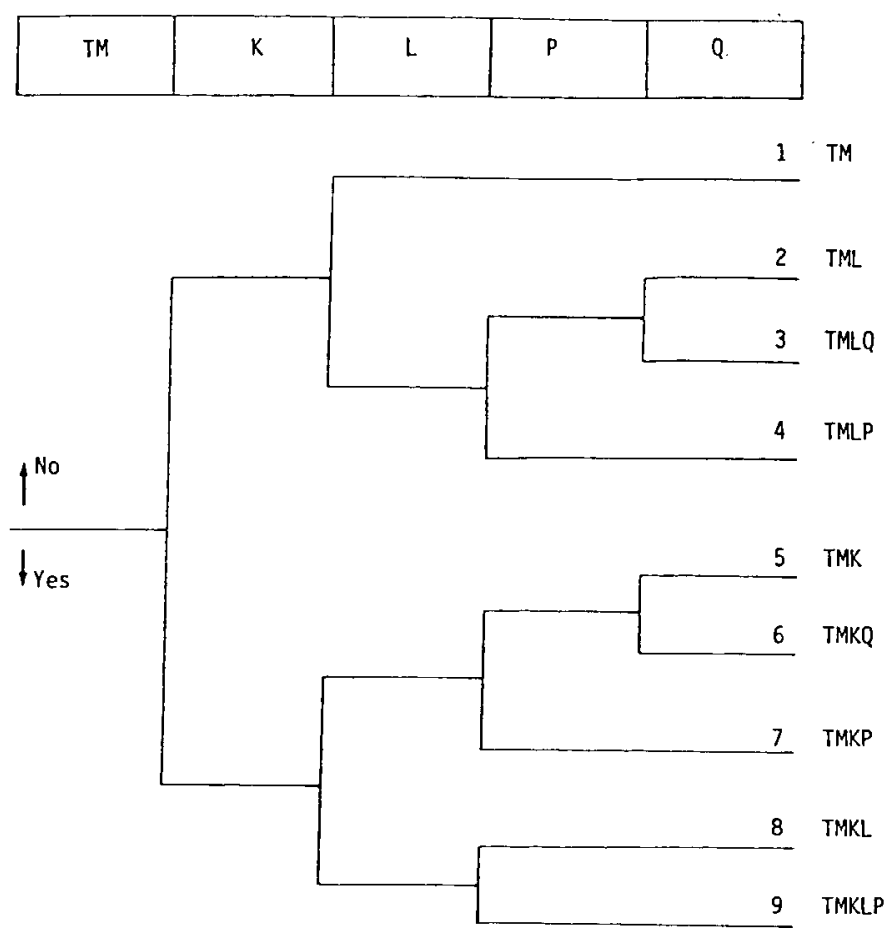

Figure 3.21. Loss of feedwater event tree adapted from the generic event tree for transients given in WASH- 1400 


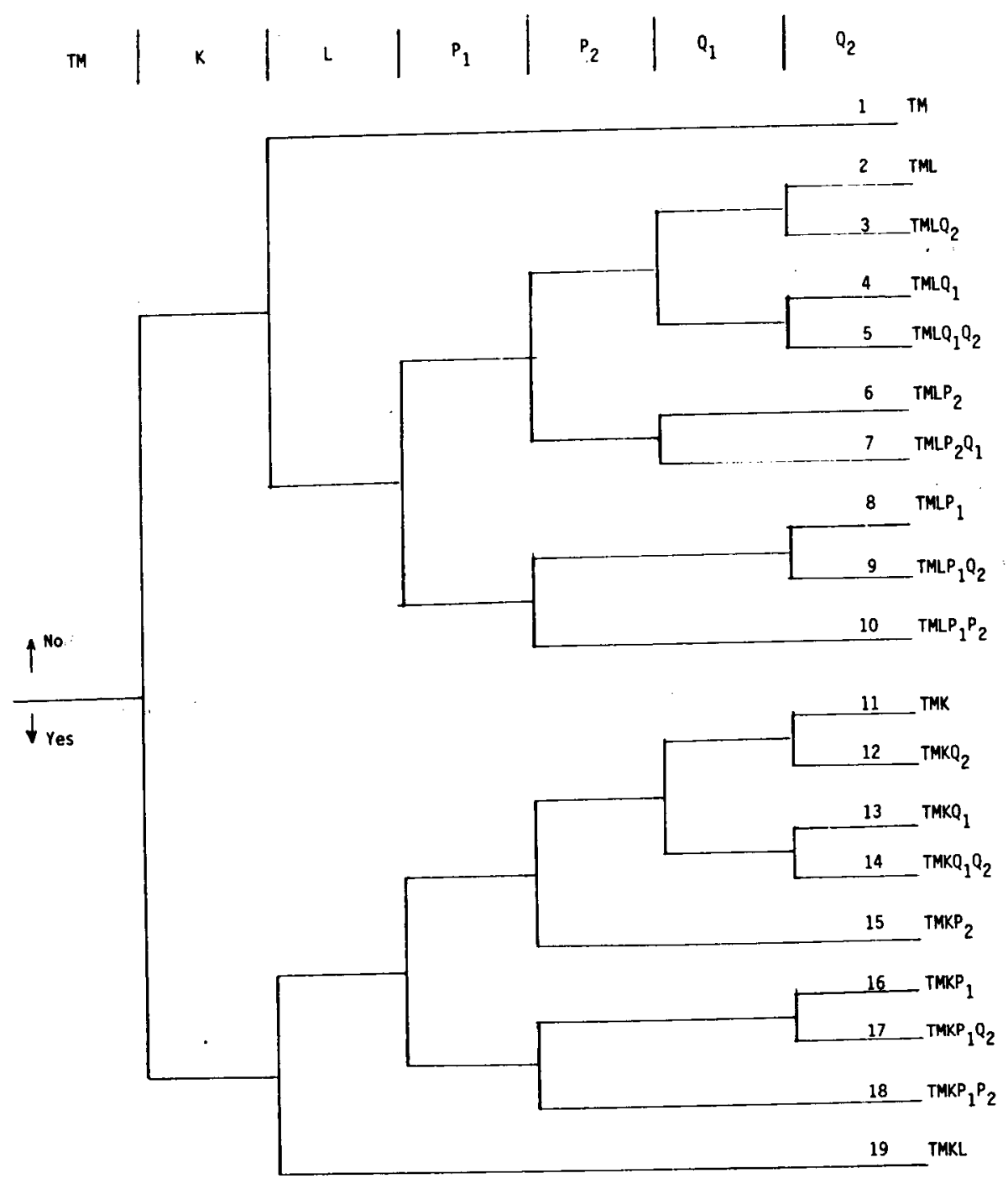

Figure 3.22. Loss of a11 feedwater event tree 
accounted for by loss of offsite power. Data collected by Electric Power Research Institute indicates that there have been eight cases of total loss of main feedwater (all loops) in 101 reactor-years of operation. (19) The largest number of loss of feedwater events occurs during the first year of operation. Six LOF events occurred during the first year of operation for 23 plants.

$\therefore$ Mean Frequency of LOF event $=0.08 /$ reactor year (EPRI estimate).

The above mean frequency is an underestimate since it does not include the loss of all electrically driven main feedwater pumps that results from loss of station power. The Surry power plant, which has been chosen for this study, has electrically driven main feedwater pumps. Data(19) shows that there has been 32 cases of loss of station power over a period of 101 reactor-years. Thus, the amended estimate is

Amended mean frequency of LOF event $=0.40 /$ reactor year.

The loss of all condensate pumps would also lead to loss of all main feedwater but the EPRI data(2) shows that there has been no incident of loss of all condensate pumps.

The above estimate is not utilized in this study since no extensive data evaluation was done for the other events. Thus, to be conservative in lieu of the uncertainty existing in the other event frequencies the WASH-1400 LOF event frequency of $3 /$ reactor-year is chosen. 
TABLE 3.6

FREQUENCY OF VARIOUS EVENT. SEQUENCES

IN LOSS OF FEEDWATER TRANSIENTS

\begin{tabular}{|c|c|c|c|}
\hline & \multicolumn{2}{|c|}{$\begin{array}{l}\text { Frequency of Events } \\
\text { per Reactor Year }\end{array}$} & \multirow[b]{2}{*}{ 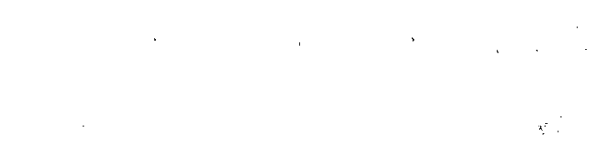 } \\
\hline Event & Expression & Point Estimate & \\
\hline 1 & $P_{T M}$ & 3 & \\
\hline 2 & $\mathrm{P}_{T M} \mathrm{P}_{\mathrm{L}}$ & $4.2 \times 10^{-4}$ & \\
\hline 3 & $\mathrm{P}_{T M} \mathrm{P}_{L} \mathrm{P}_{\mathrm{Q}_{2}}$ & $4.2 \times 10^{-6}$ & Input Probabilities/Frequencies \\
\hline 4 & $P_{T M} P_{L} P_{Q_{1}}$ & $4.2 \times 10^{-6}$ & $\mathrm{P}_{\mathrm{TM}}=3 /$ reactor-year \\
\hline $\begin{array}{l}5 \\
6\end{array}$ & $\begin{array}{l}P_{T M} P_{L} P_{Q_{1}} P_{Q_{2}} \\
P_{T M} P_{L} P_{P} P_{2}\end{array}$ & $\begin{array}{l}4.2 \times 10 \\
1.3 \times 10^{-7}\end{array}$ & $P_{K}=3.6 \times 10^{-5} / D$ \\
\hline 7 & ${ }^{P} T M^{P} P^{P} P_{2}^{2} P_{1}$ & $1.3 \times 10^{-9}$ & $L=\begin{array}{l}1.4 \times 10^{-4} \\
0.04 / D \text { for end event } 19\end{array}$ \\
\hline $\begin{array}{l}8 \\
9\end{array}$ & $\begin{array}{l}\mathrm{P}_{T M} \mathrm{P}_{L} \mathrm{P}_{\mathrm{P}_{1}} \\
\mathrm{P}_{T M} \mathrm{P}_{L} \mathrm{P}_{\mathrm{P}_{1}} \mathrm{P}_{\mathrm{Q}_{2}}\end{array}$ & $\begin{array}{l}8.8 \times 10^{-6} \\
8.8 \times 10^{-8}\end{array}$ & $P_{1}=2.1 \times 10^{-2} / D$ \\
\hline 10 & $\mathrm{P}_{T M} \mathrm{P}_{L} \mathrm{P}_{\mathrm{P}_{1}} \mathrm{P}_{\mathrm{P}_{2}}$ & $2.6 \times 10^{-9}$ & $P_{2}=3 \times 10^{-4} / D$ \\
\hline 11 & $P_{T M}^{\prime} P_{K}$ & $1.1 \times 10^{-4}$ & $Q_{1}=Q_{2}=10^{-2} / 0$ \\
\hline 12 & $\mathrm{P}_{T M} \mathrm{P}_{K} \mathrm{P}_{\mathrm{Q}_{2}}$ & $1.1 \times 10^{-6}$ & \\
\hline 13 & ${ }^{P_{T M}}{ }^{P} K^{P} Q_{1}$ & $1.1 \times 10^{-6}$ & \\
\hline 14 & ${ }^{P_{T M}}{ }^{P} K^{P} Q_{1}{ }^{P} Q_{2}$ & $1.1 \times 10^{-8}$ & \\
\hline 15 & $\mathrm{P}_{T M} \mathrm{P} \mathrm{P}^{\mathrm{P}_{2}}$ & $3.2 \times 10^{-8}$ & \\
\hline 16 & $P_{T M} P_{K} P_{P_{1}}$ & $2.3 \times 10^{-6}$ & \\
\hline 17 & $P_{T M}{ }^{P} K^{P} P_{1} P_{Q_{2}}$ & $2.3 \times 10^{-8}$ & \\
\hline 18 & ${ }^{P_{T M}}{ }{ }{ }^{P P_{1}}{ }_{1}{ }^{P_{2}}$ & $6.8 \times 10^{-10}$ & \\
\hline 19 & $P_{T M} P_{K} P_{L}$ & $2.3 \times 10^{-6}$ & \\
\hline
\end{tabular}


3.6.2.2 Probability of Failure of the Reactor Protection System (RPS) per demand: event $(K)$ :

Failure of the RPS is failure to scram. The injection of boron by the Chemical and Volume Control System to make the reactor subcritical is not sufficiently rapid to shutdown the reactor for the LOF transient. The probability of failure to scram is chosen in this study as $3.6 \times 10^{-5} /$ demand as per WASH-1400.

\subsubsection{Unavailability of Auxiliary Feedwater System (AWFS): event (L):}

The AFWS unavailability has been assessed in WASH-1400(1) for three separate initiating events, (i) a small pipe break, (ii) loss of offsite power, and (iii) high energy break. For the first two events the system unavailability were estimated for the first eight hours following the incident and from eight hours to twenty-four hours following the transient. The unavailability of AFWS used in this study combines the probability of failure for the first two initiating events in the time frame of 0-8 hours following the incident. The value chosen here is $1.4 \times 10^{-4} /$ demand with an error factor of 20. For the LOF event followed by a scram only 1 of 3 auxiliary feedwater trains are necessary. However, for the LOF event not followed by a scram it is assumed that all three auxiliary feedwater trains are necessary. Thus, failure of one or more auxiliary feedwater trains would be termed as AWFS failure.

If $p$ is the probability of failure per demand of one auxiliary train and if it is assumed that the probability of failure is equal for all three auxiliary feedwater trains then the probability of AFWS failure is equal to 


$$
\sum_{i=1}^{3}\left(\begin{array}{l}
3 \\
i
\end{array}\right) p^{i}(1-p)^{3-i}=1-(1-p)^{3}
$$

From Reference 20 the probability that one train of AFWS is lost is given to 0.04. This is interpreted as one of three AFW trains failing. Therefore $p$ is 0.014 .

3.6.2.4 Probability of Failure of a Relief Valve from Opening: event $\left(P_{1}\right)$ :

The probability of failure to open for a relief valve from Reference 21 is $1.075 \times 10^{-2} /$ demand. There are two relief valves connected to the pressurizer. Therefore, the probability that one out of two valves failing to open is given by

$$
2 p(1-p)=2.1 \times 10^{-2} / \text { demand }
$$

where $p=1.075 \times 10^{-2} / 0$.

3.6.2.5 Probability of Failure of Safety Valves from Opening: event $\left(P_{2}\right)$ :

The failure probability of safety valves from opening is given in Reference 22 as $3 \times 10^{-5} / D$. A study of the testing schedule of safety valves as given in Reference 6 indicates that the number of test demands accumulated upto this date does not support the above estimate. Therefore, the above estimate was increased by a factor of 10 in this work. 
3.6.2.6 Failure Probability of the Relief and Safety Valves to Reclose: Events $\left(Q_{1}, Q_{2}\right)$

The failure probability derived in Reference 23 from PWR operating experience is $10^{-2} /$ demand. It is assumed that the failure probability to reclose is the same for the relief valve as well as the safety valve. This is a conservative assumption since typically safety valves are more likely to reclose than relief valves. 


\subsection{RADIONUCLIDE INVENTORY SURVEY}

This chapter describes the survey performed to assure that no major sources of radioactivity have been overlooked in the Class 3-8 accident analysis of pressurized water reactors. The objective of this assessment was to complement the analysis of specific Class 3-8 accident scenarios described in Section 3 and the analysis of operating experience described in Section 2.

Figure 4.1 shows a plot plan of the Surry nuclear power plant. From this plan, the following buildings and tanks were defined that could contain radioactive components:

- Reactor Containment

- Auxiliary Building

- Turbine Building

- Fuel Building

- Decontamination Building

- Boron Recovery Tanks
- Primary Water Storage Tanks

- Condensate Storage Tanks (100,000 gal)

- Refueling Water Storage Tanks

- Waste Gas Decay Tanks

- Low Level Waste Storage Pad

- Other Condensate Storage Tanks

- Waste Drum Storage and Spent Filter Storage Area

The survey was conducted by listing all components that could be radioactive sources for each of the above buildings or tanks. The range of activity levels available for release were estimated for each component (sometimes on a comparative basis as being either larger or smaller than some other

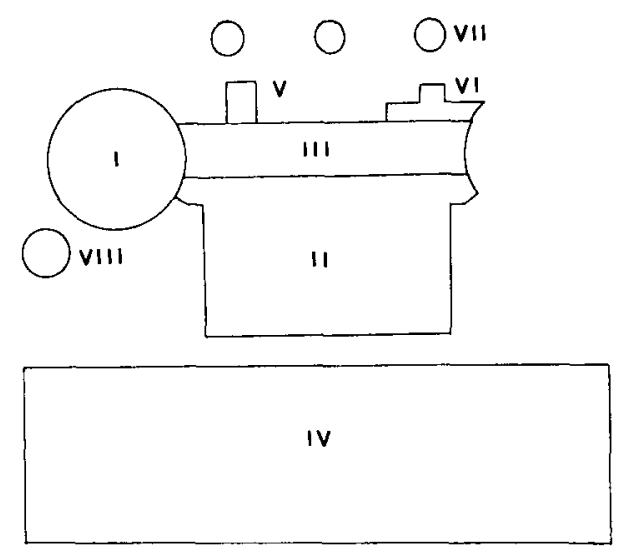

1. REACTOR BUILDING V. BORON RECOVERY PUMP ROOM

i1. AUXILIARY BUILDING VI. DECONTAMINATION BUILDING

III. FUEL BUILDING VII. BORON RECOVERY TANKS

IV. TURBINE BUILDING VIII. RWST

Figure 4.1. Schematic plot plan for Surry 
component). The initial set of sources in a building was pruned to a smaller set by considering only those components with potential radioactive levels such that they might be significant contributors to the risk curve if all of the activity were released. A further pruning of the subset was achieved by eliminating those components that might be significant sources, but for which multiple barriers to direct release exist. Examples of such barriers could be, for liquid releases, sumps nomally drained to the liquid waste treatment system or, for gaseous releases, building filters that would normally be in operation. Only those potential radioactive sources where the barriers could reasonably be bypassed or defeated remained after the barrier analysis. Components in this category were analyzed in more detail.

\subsection{PLANT SURVEY AND RELEASE MECHANISMS}

A brief description is given here of those components and release mechanisms which were deemed to merit further study. Appendix $N$ gives a detailed description of the parts of the plant considered and of the barrier analysis for each component.

1. Pressurizer relief tank. The purpose of this tank is to condense any steam discharges from the pressurizer safety/relief valves. Failure of the rupture discs would discharge steam within containment.

2. Regenerative heat exchanger. Tube leaks would allow charging water to mix with the letdown flow.

3. Excess letdown heat exchanger. Tube leaks would contaminate the component cooling water. This would be detected by radiation monitors or by the temperature change. 
4. Primary drain transfer tank subsystem. Leakage from tanks, piping, etc., would collect in the containment sump.

5. Residual heat renoval system heat exchangers. Tube leaks would contaminate the component cooling water system. This would be detected by radiation monitors. Leakage from piping, valves, etc., would collect in the containment sump.

6. Neutron shield tank. This tank experiences a high neutron flux since it surrounds the reactor vessel. Leakage would collect in the containnent sump.

7. Volume control tank. Rupture of the tank would release the contents within the auxiliary building. The relief valves vent to the gas waste processing system.

8. Mixed bed and cation demineralizers. Rupture of the tanks would release the contents within a concrete vault. During the flushing of spent resins, a spill would drain to the auxiliary building sump.

9. CVCS filters. If leaks develop, the filter vessels can be bypassed.

10. Non-regenerative heat exchanger and seal water heat exchanger. Tube leaks would contaminate the component cooling water. This would be detected by radiation monitors.

11. Gas stripper surge tank. Leakage from the tank would be within the auxiliary building.

12. Boron recovery tanks. Leakage from the tanks could release noble gases directly to the atmosphere.

13. Boron test tanks. These tanks are smaller than the Boron recovery tanks and do not contain significant amounts of noble gases. 
14. Evaporator bottoms in liquid waste processing system. There are two valves which, if inadvertently open, would release evaporator bottoms. However, the liquid would drain to the Vent and Drain system thence back to the Liquid Waste Processing system.

15. Hydrogen Recombiner. Two redundant $H$ analyzers maintain the hydrogen concentration below the flammability limit. The gas stream is automatically diluted with nitrogen if the hydrogen concentration rises.

16. Steam Generator blowdown tank. This tank receives blowdown from the secondary side of the steam generators. The tank is open to the atmosphere and normally drained to the circulating water system.

17. Turbine building. Most fission and corrosion products plate out in the condenser, moisture separator and reheater. The total activity plated out is relatively insignificant.

18. Tritium releases. The spent fuel storage pool, the refueling water storage tank, and the primary water storage tank may contain significant amounts of tritium. Liquid pathways have not been exarnined in detail in this report. In the spent fuel pool loss of cooling accident analyzed in Chapter 3 the affects of tritium vapor were not calculated.

19. Decontamination building. Any liquid spills would drain into the sumps, thence to the liquid waste processing system. The ventilation vent contains a radiation monitor which can divert the flow through charcoal filters. A possible accident scenario is a rupture of the fluid waste treatment tank during transport of a spent resin cask. 


\subsection{RESULTS OF SURVEY}

No major new accident scenarios were identified during the detailed examination of the plant drawings in the FSAR. Most components containing relatively high amounts of radioactivity were protected by multiple barriers from an accidental release to the environment. The following scenarios are among the more significant of those identified.

1. Failure of rupture discs in the pressurizer relief tank and failure to isolate contaiment.

2. Spill of spent resins during flushing.

3. Leakage of Boron recovery tanks when the gas stripper is being bypassed.

4. Tritium releases from the spent fuel pool.

5. Rupture of fluid waste treatment tank during transport of a spent fuel cask.

On the basis of a very conservative preliminary analysis, the risk from these events is in Section 3. If a more realistic less conservative analys is were made, one could expect a significant reduction in the risk estimates. Those major sources identified by this radionuclide inventory survey were then subjected to a boundary analysis. This was accomplished by applying broad probabilities to the failure of each boundary that prevented the release of the inventory to the biosphere. If a high failure probability and a high radioactive inventory were simultaneousiy found, the sequence was analyzed in greater detail and appears up in Sections 2 and 3 of this report. 


\subsection{RESULTS}

In Figure 5.1 the weighted complementary cumulative distribution functions for each of the sequences analyzed are plotted with LER releases and compared to the results of WASH-1400 for meltdown accidents. Table 5.1 describes each specific sequence shown. Although there are some uncertainties associated wi th the treatment of the LER data, related to the accuracy wi th which releases are reported and the applicability of the past performance of reactors to the prediction of future perfomance under changing licensing conditions, these data do represent actual experience. Larger uncertainties are associated with the extrapolation of actuarial data to larger consequences and smaller probabilities. Although it is difficult to set an absolute upper hound on the consequences of class 3 to 8 accidents it is interesting to examine two limiting inventories: the maximum primary coolant activity and the maximum waste decay tank inventory. At a $1 \%$ clad defect level the total inventory of I-131 in the primary system (without spiking) is $70 \mathrm{Ci}$. The maximum inventory of $\mathrm{Xe}-133$ in a waste decay tank is $43,800 \mathrm{Ci}$ which when weighted corresponds to $14 \mathrm{Ci}$ of I-131 equivalent. Thus the probability of Class 3 to 8 accidents with consequences greater than 100 curies of equivalent I-131 is expected to be exceedingly small. The accidental risk spectrum can therefore be broken down into three regions: the region corresponds to less than $1 \mathrm{Ci}$ or I-131 equivalent, which is covered by data; the region from $1 \mathrm{Ci}$ to 10 or $100 \mathrm{Ci}$ of I-131 equivalent, which is controlled by Class 3 to 8 accidents but which must be examined by the extrapolation of actuarial data or by engineering analyses of specified accident sequences; and the region greater than 10 or $100 \mathrm{Ci}$ of I-131 equivalent, which is controlled by Class 9 accidents and which is examined by engineering analyses. 


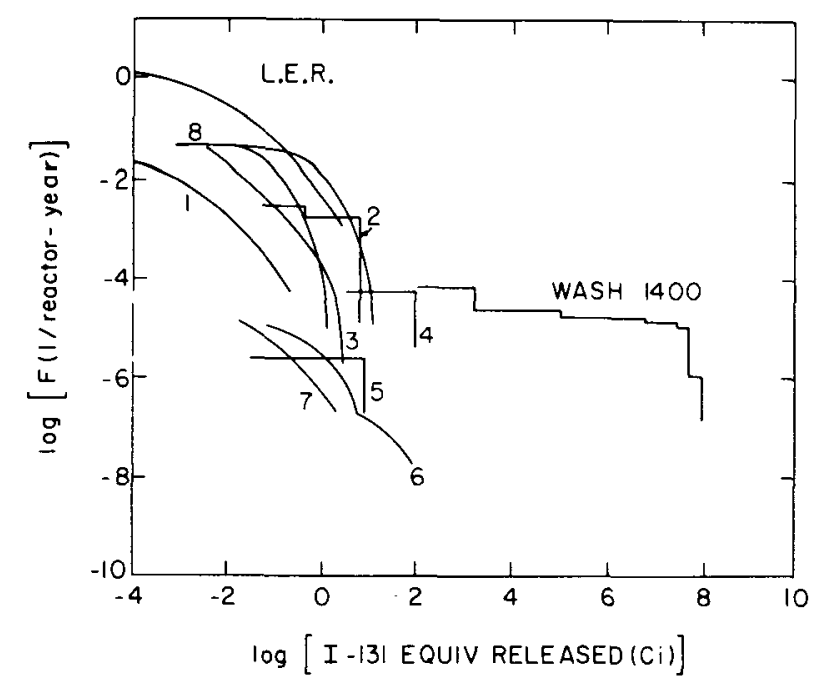

Figure 5.1. The weighted risk calculated for accident scenarios compared to the LER data and the results reported in WASH-1400. The curves for the accident scenario are: (1) loss of offsite power, (2) fuel assembly drop within containment, (3) fuel assembly drop in spent fuel storage pool, (4) steam line break, (5) core barrel drop, (6) steam generator tube rupture, (7) LOSP of cooling in spent fuel storage poo1, (8) decay tank

TABLE 5.1

PWR ACCIDENT SCENARIO RANKING

Index (Expected

Accident

Gas decay tank

Fuel handling within containment

SLB with SGTR

Fuel handling within SFP

Loss of offsite power

Core barrel drop

SGTR with LOP

SFP loss of cooling
Equivalent 1-131 Release

- Ci/Reactor $\mathrm{Yr}$ )

$3(-2), 2(-3)$

$9(-3)$

$5(-3)$

$2(-3)$

$1(-4)$

$1(-5)$

$9(-6)$

$2(-6)$
Figure 5.1

Notations

8

2

4

3

1

5

6

7 
In Table 5.1 the distribution functions for each of the specified accident sequences have been integrated to obtain the expected release per year. The sequences have been ranked in importance accordingly. Fram inspection of Figure 5.1 it does not appear that enough specific accident sequences have been analyzed to obtain an envelope to fill in the risk curve in the region of 1 to $100 \mathrm{Ci}$. It is concluded that the use of specific accident sequence analyses, based on engineering calculations, is not practical in this intermediate area. The number of evaluations needed to even hope to identify small sections of the risk envelope in the range of 1 to $100 \mathrm{Ci}$ is large and requires a manpower investment, beyond the scope of this program, with no guarantee of results. Instead it appears that an extrapolation of the actuarial, LER, data will produce a usable upper bound. It is interesting to examine the potential impact on risk of various extrapolations of the actuarial data through this region. In Figure 5.2 three possible extrapolations are shown which involve varying degrees of conservatism. The Curve 1 assumption that the probability does not decrease in this region is clearly an upper bound. In Table 5.2 the results of integrating the different regions of the risk curve are tabulated. Also shown for comparative purposes is the weighted annual release of radioactivity from an average PWR from nomal operations. Except for the very conservative extrapolation of Class 3 to 8 data in Curve 1 , the expected release fram class 3 to 8 accidents as defined in this report is small in comparison to normal operational releases and very small in comparison to Class 9 accidents. For a detailed discussion of all conclusions of this risk assessmentm, reference Section 6 of this report. 


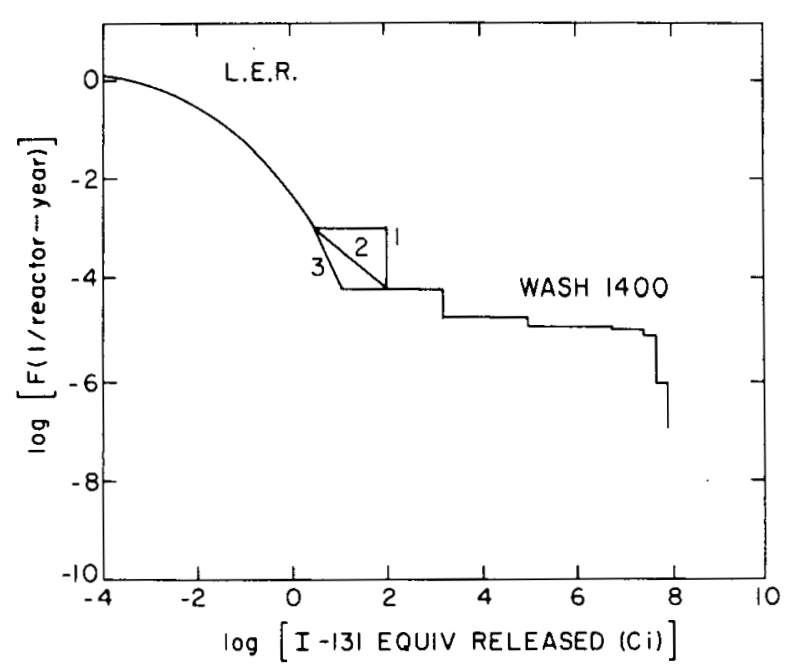

Figure 5.2. Extrapolation of the weighted risk curves in the regime intermediate between the LER data and WASH-1400

\section{TABLE 5.2}

COMPARISON OF THE EXPECTED EQUIVALENT

I-131 RELEASE FOR PWRS

Expected Equivalent $[-13]$ Release (Ci/Reactor $Y r$ )

\section{Class 3-8 accidents}

Actuarial $(<2.5 \mathrm{Ci})$

.04

Extrapolated $(2.5-100 \mathrm{Ci})$

.14

Curve 1*

.06

Curve 2*

.05

Curve $3^{*}$

Class 9 accidents (>100 Ci)

Normal PWR operational releases**

1.7

*From, Figure 5.2

**"Radioactive Material Released fron Nuclear Power Plants in 1974," Nuclear Safety, Volume 18, No. 1, 1977. 


\subsection{CONCLUSIONS}

The risk from Class 3 to 8 accidents in a pressurized water reactor have been investigated using methods similar to those used in WASH-1400. The results of the study must be considered preliminary since it was not possible within the prescribed scope of the effort to examine accident sequences or actuarial data to the extent that would be necessary to make definitive statements on risk. In particular, the uncertainty bounds associated with the estimated risk curve have not been detemined al though some insights into their magnitude have been explored. The results that have been obtained, however, do strongly suggest the following conclusions.

1. Class 3 to 8 accidents, as reviewed in this report, provide a small contribution to risk in comparison with Class 9 accidents and normal plant releases. This conclusion is shown clearly in Table 5.2 and is relatively insensitive to uncertainties in the risk estimates. Some important inferences to reactor safety research and reactor regulation can be drawn from this conclusion.

i. The development of improved systems to reduce the consequences of Class 3 to 8 accidents is probably unwarranted. If it were felt that the risk of these accidents were unacceptable, efforts should first be directed at reducing nomal releases or in reducing the risk of class 9 accidents.

ii. The importance of Class 3 to 8 accidents to the regulatory and licensing process is not derived from any significant risk to the public 
from the consequences of these accidents. In evaluating the response of the plant to class 3 to 8 accidents the focus of concern should be on the margin that exists between a controlled accident and degeneration of the accident into a class 9 accident with core damage above that as considered in this report.

2. LER data can be used to examine a major part of the risk of class 3 to 8 accidents. The use of the LER data for estimating risk was hampered considerably by lack of uniformity in reporting releases. More effort is warranted in evaluating the accuracy of the data and in the use of the data to extrapolate to lower probabilities in combination with engineering analyses than could be undertaken in this project. A major portion of the risk curve for. Class 3 to 8 accidents was developed from these data, however.

\section{Partial failure analysis is a useful extension of WASH-1400 risk} methodology. The binomial characterization of failures is not adequate for the analysis of all accident events. The methods of partial failure analysis were found to be a practical means of analyzing events which have a spectrum of potential outcomes. By separating the variation in parameters from the uncertianty associated with ignorance, it was possible to develop uncertainty bounds for the risk curves which were developed through this method of analysis.

4. Noble gases are as important as halogens to the consequences of Class 3 to 8 accidents. In general the analysis of class 3 to 8 accidents in safety analys is reports is very conservative. This is usually particularly true for iodine releases. Although models developed in this study indicated iodine spiking to be a major contributor to iodine release, in most accidents the amount of iodine which would be expected to escape on a realistic basis is smal 1. 
5. The 10CFR50 accident classification scheme is reasonably representative. Although considerable effort was devoted to identifying new accident sequences or variations of old accident sequences, only a few new sequences were identified of potential significance.

6. Weighting of radionuclides is essential to the interpretation of risk. Although the factors used for weighting the importance of radionuclides cannot be determined precisely, they do add perspective to the results. In many accidents organic iodine was found to dominate the release on a curie basis but when weighted became comparatively insignificant. The importance of noble gases is also over emphasized on a curie basis. The significance of class 9 accidents to the risk of nuclear power reactors becomes even more apparent, nowever, when the releases are weighted as demonstrated in Table 5.2. 
APPENDIX A

RESULTS FROM WE IBULL CURVE

FITTING OF LER DATA

\section{A.1 The Weibull Distribution}

In order to use the data in further studies, it is convenient to describe it in terms of a parametric distribution with a small number of parameters. It may then be possible to relate these parameters to basic factors which affect the releases (24). A code WBFIT has been developed to fit the data to a Weibull distribution. The code and statistical techniques are described in Appendix B. Here we shall briefly describe the procedures adopted.

The observed data, the number of curies released in the reportable incidents, spans several orders of magnitude, is always positive and is characterized by a distribution with a long tail. A priori, any theoretical distribution with these characteristics could be examined, eg. log nomal, gamma or Weibu11. At present the Weibull distribution has been selected, since it has many convenient features and is characterized by a flexible distribution shape. We will test the Weibull assumption with statistical hypothesis tests. The Weibull distribution involves two parameters, $\beta$, a shape parameter, and $\delta$, a scale parameter. It does not seem necessary, at present, to use the three parameter Weibull distribution. The probability density, $f(x)$, for the Weibull distribution is

$$
f(x)=\left(\frac{\beta}{\delta}\right)\left(\frac{x}{\delta}\right)^{\beta-1} \exp \left[-\left(\frac{x}{\delta}\right)^{\beta}\right]
$$


and the complementary cumulative distribution is

$$
F(x)=\exp \left[-(x / \delta)^{\beta}\right]
$$

Several estimators for the parameters $\beta$ and $\delta$ have been suggested. The maxinum likelihood estimator has small mean square error, is asymptotically efficient (25), and tables are available to correct for the bias in small samples, so this method has been selected. Code WBFIT solves the implicit equations for $\beta$ and $\delta$ by the Newton-Raphson method. Tables(26) are incorporated in the code to correct for bias and to calculate the 5 percent and 10 percent upper and lower confidence limits for $\beta$ and $\delta$.

WBFIT performs several tests on the goodness of the fit between the observed data and the distribution based on the estimated parameters. The data and theoretical curves are al so plotted in order that a visual judgement may be made concerning the choice of the theoretical distribution. The Chi Square test is applicable to a composite hypothesis, that is a distribution function which involves estimated parameters. The data are grouped into intervals and the observed number of data points in each interval is compared with the number predicted on the basis of the theoretical distribution. The test requires sample sizes larger than about 15. Since many of the samples are small, an additional test is required for samples smaller than 16 . Mann et al (27) constructed a test specifically for the Weibull distribution. The code uses the tables published by Mann et al(27). The significance level based on the Kolmogorov-Smirnov(25) distance is calculated. This test is a classical test for a completely specified distribution and may be used if the Weibull parameters are preassigned. Further details are given in the report on WBFIT (28). 


\section{A.2 Comparison of Data with a Weibull Distribution}

The code WBFIT has been used to calculate the paraneters of a wejbull distribution and to check the adequacy of the fit for the data on all of the releases and for the data broken down among the specified categories used. More details of the code are given in Appendix B. For brevity, we shall give only a selection of the results here, the complete set of tables is available upon request. WBFIT produces two different plots to provide a visual estimate of the appropriateness of the choice of a Weibull distribution. The first plot used Weibull probability paper, with the $y$ axis $\ln (\ln (1 / F))$ and the $x$ axis In(activity). Under this transformation, a.. Weibull distribution becomes a straight line. Any marked departures of the underlying distribution from the general shape of a Weibull distribution will show up, e.g., the data may tend to follow a curve or may break into subsets rather than lying along one line. This plot is also useful in detecting outliers. The second plot consists of $F(x)$ versus $x=\log ($ activity $)$. This plot allows one to assess how well the theoretical distribution describes the observed data in different ranges. The information from the plots complements the results of the statistical goodness of fit tests described in this section. These statistical tests assess quantitatively the overall adequacy of the hypothetical distribution to describe the data, but do not give any clues as to why the fit may be poor.

Figure A1 shows the two plots for all the releases. The fit of the hypothetical distribution is better than the $20 \%$ significance level using the Chi Square test. Figure. Ala is the plot on Weibull paper. Apart from the 5 largest releases, the other data points lie close to the straight line. The 4 largest of these 5 releases are included in the data sample since they are reported in the LER file. However, these releases are cumulative releases 
over a quarter in contrast to the other releases which occur over a time span of at most a few hours. Including these releases causes the theoretical distribution to be chosen so that it has a longer tail than if those 4 events were excluded from the study.

Table A1, shows, for the major systems, the estimated parameters $\beta$ and $\delta$, together with the $95 \%$ confidence range for these parameters, and also the significance level of the goodness of fit tests. The fit is reasonable in most cases. For the primary coolant system the fit is significant at the $9 \%$ level and for the liquid radwaste system at the $6 \%$ level. Figure A2 shows the plotted curves for the gas radwaste system. There is some scatter of the points but the general description is adequate over the central part of the range as is borne out by Table Al. Figure A3 shows the plotted curves for the liquid radwaste system. Table $A 1$ indicates that the overall $f$ it is poor. In Figure $\mathrm{A} 3 \mathrm{a}$, the data would be better described by drawing two lines, one through the points with activities less that $10^{-1} \mu \mathrm{Ci}$, the other line through the remaining points. This suggests that the distribution may be better described as some other distribution or as a composite of two Weibull distributions rather than in terms of one distribution. In the high level drains the concentrations of fission products are typically of the order of $10^{-1} 1_{\mu C} i / c c$ and in the low level drains typical concentrations are $10-3 \mu \mathrm{Ci} / \mathrm{cc}$. This bimodal distribution of the inventory in the systen will affect the shape of the distribution of the releases.

The remaining figures were chosen to illustrate the effect of breaking the data down into finer categories. Figure A4a shows the difference between the liquid and gaseous releases from the liquid waste system. Figure A4b shows the influence of the cause code on the releases from the gas waste system. 


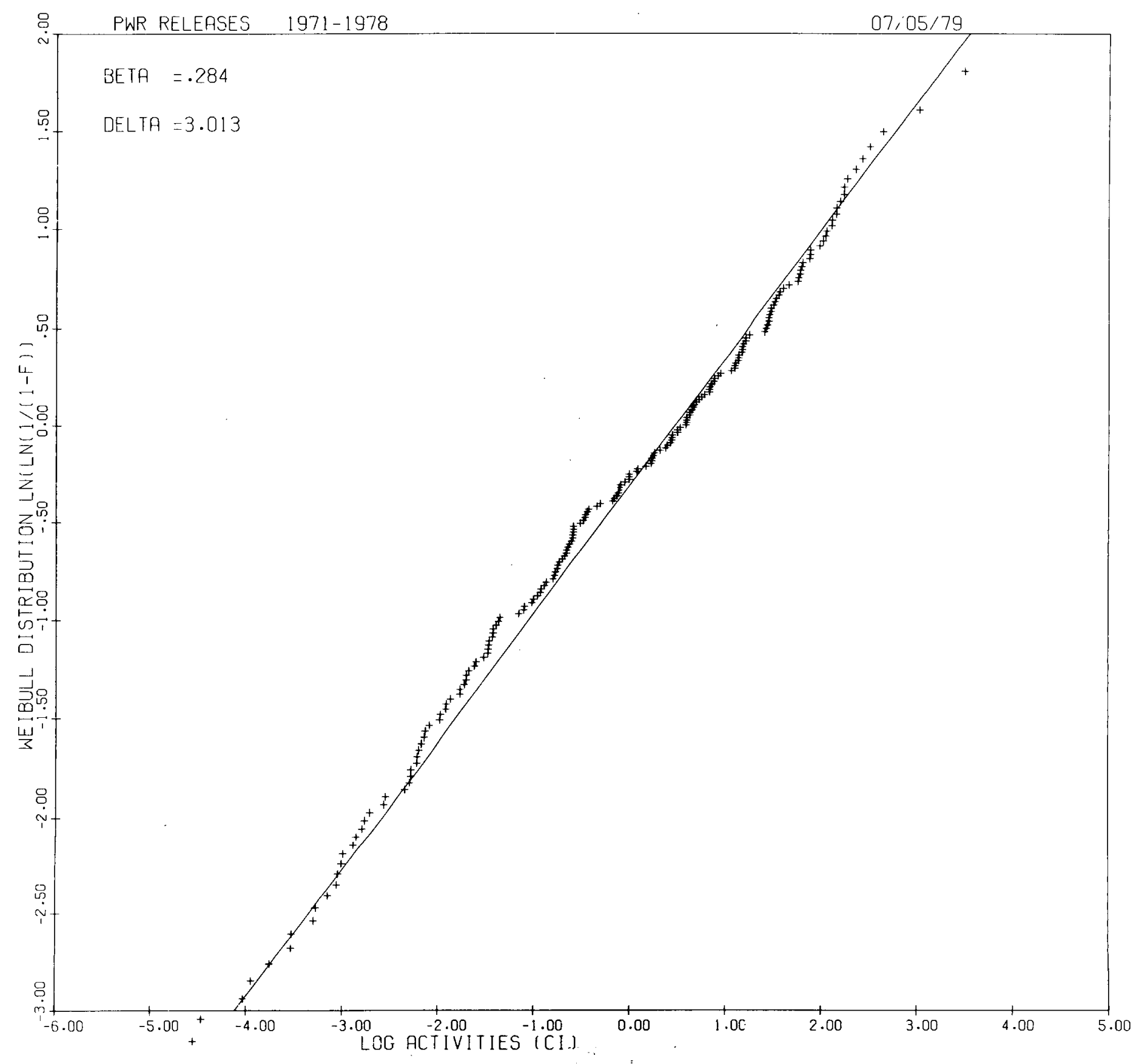

Figure Ala. Estimated CCF and the fitted Weibull CCF for the releases reported in the LER file from PWR's. : (a) Weibul1. probability paper (b) linear: plot for the $y$ axis 


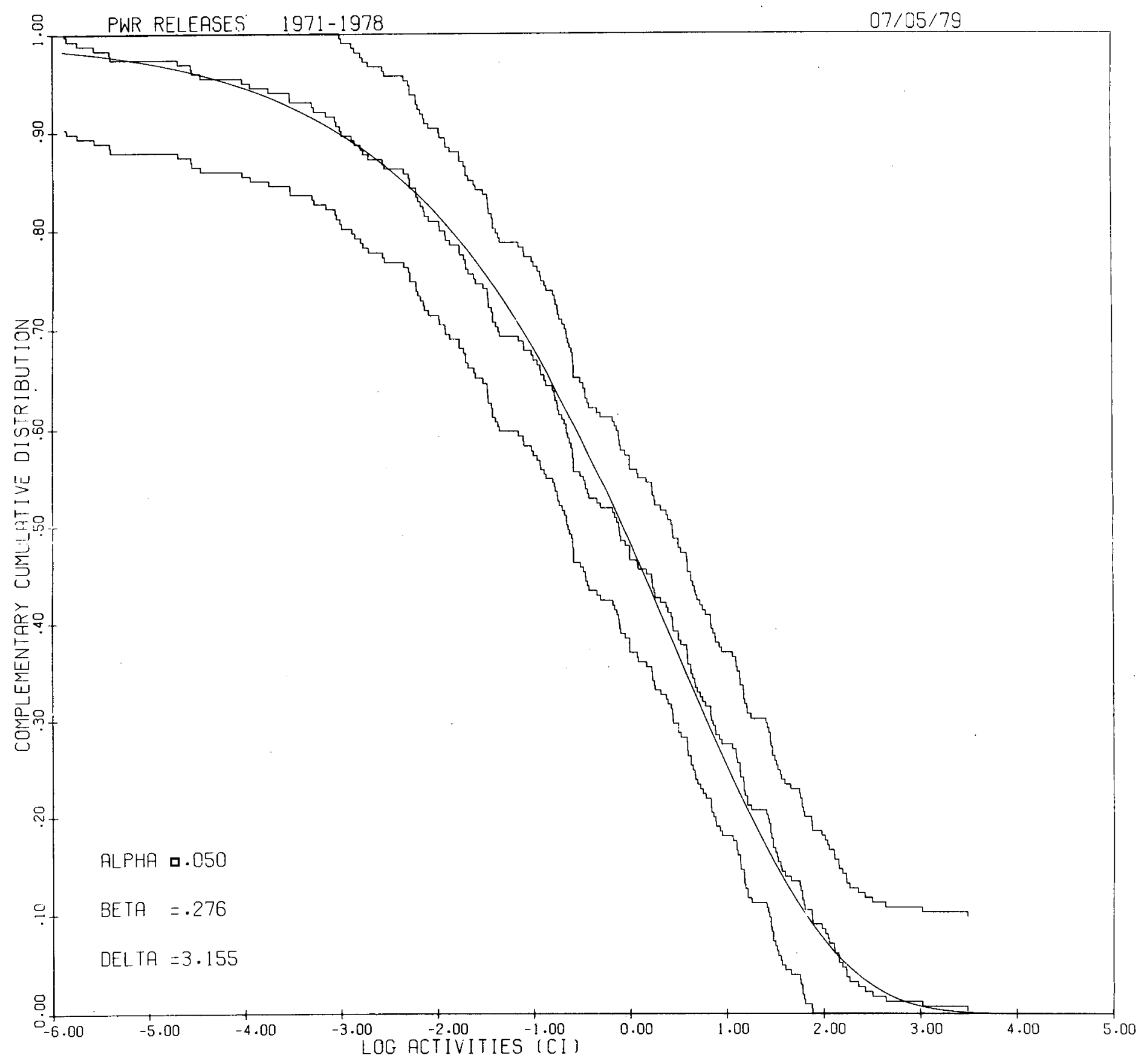

Figure Alb. Estimated CCF and the fitted Weibull CCF for the releases reported in the LER file from PWR's (a) Weibu11

probability paper (b) linear plot for the $y$ axis 


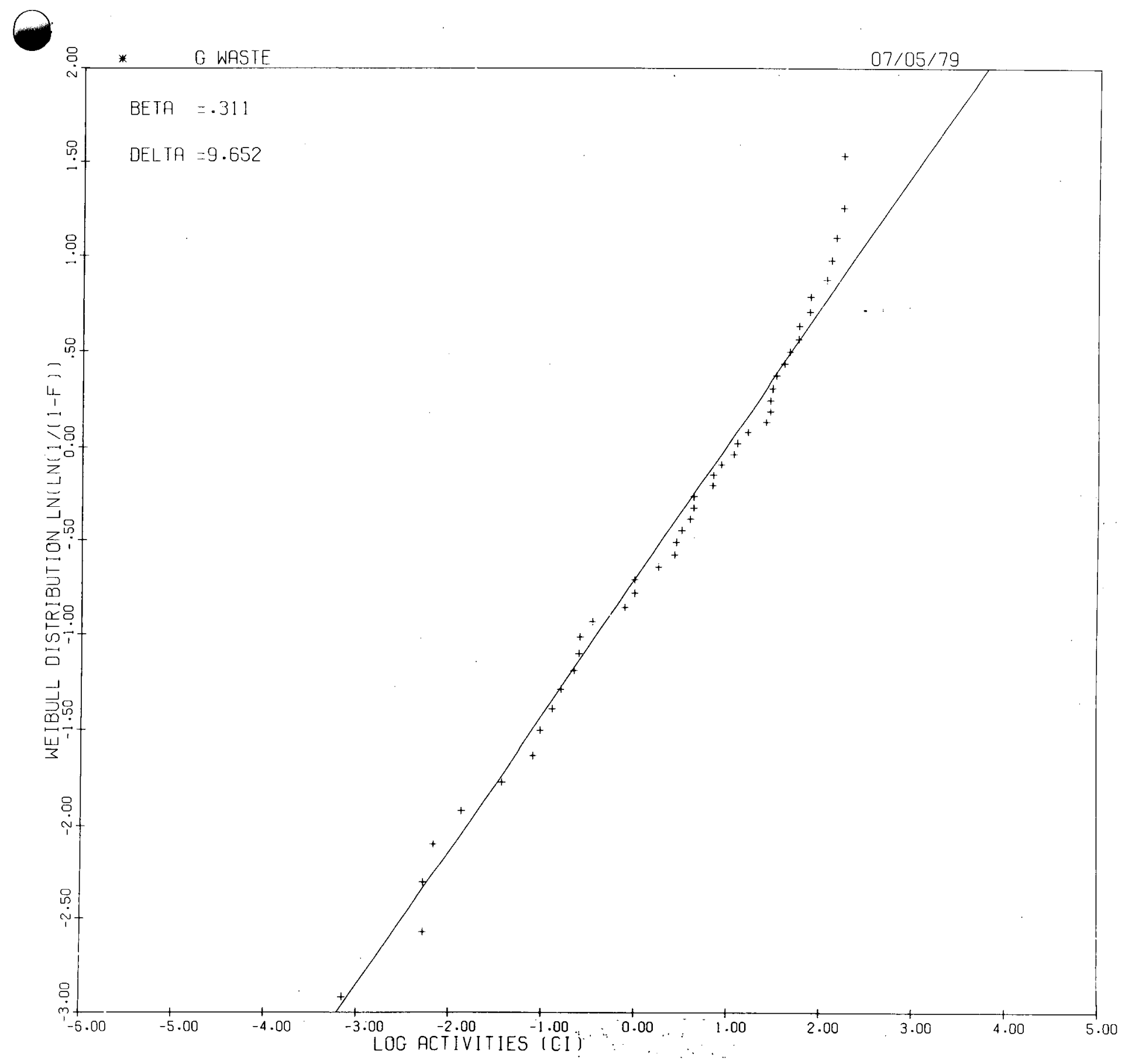

Figure A2a. Estimated CCF and the fitted Weibul1 CCF for the releases reported in the $L E R$ file from gas waste management system (a) Weibull probability paper (b) linear plot for the $y$ axis 


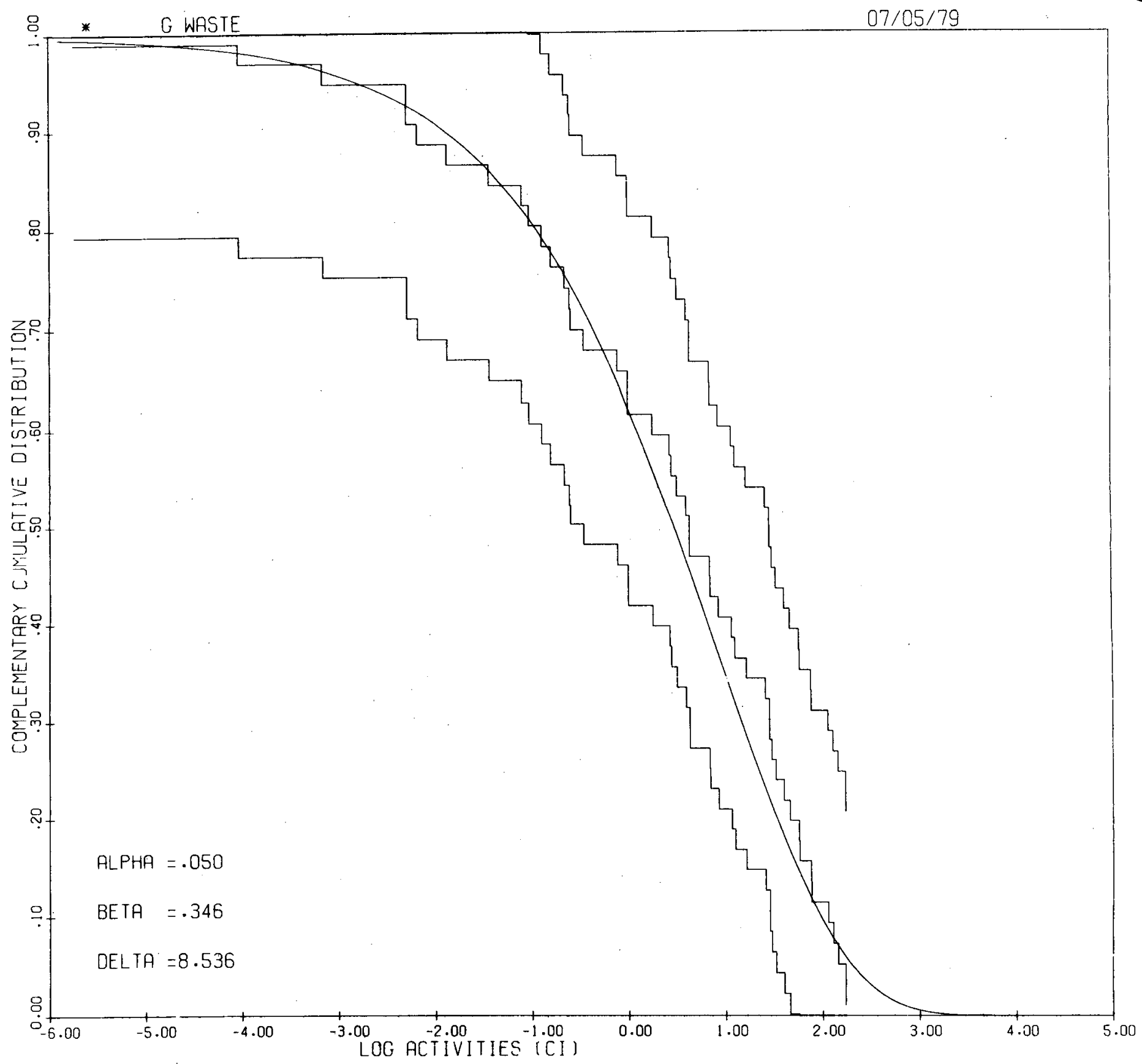

Figure A2b. Estimated CCF and the fitted Weibull CCF for the releases reported in the LER file from gas waste management system (a) Weibull probability paper (b) linear plot for the y axis 


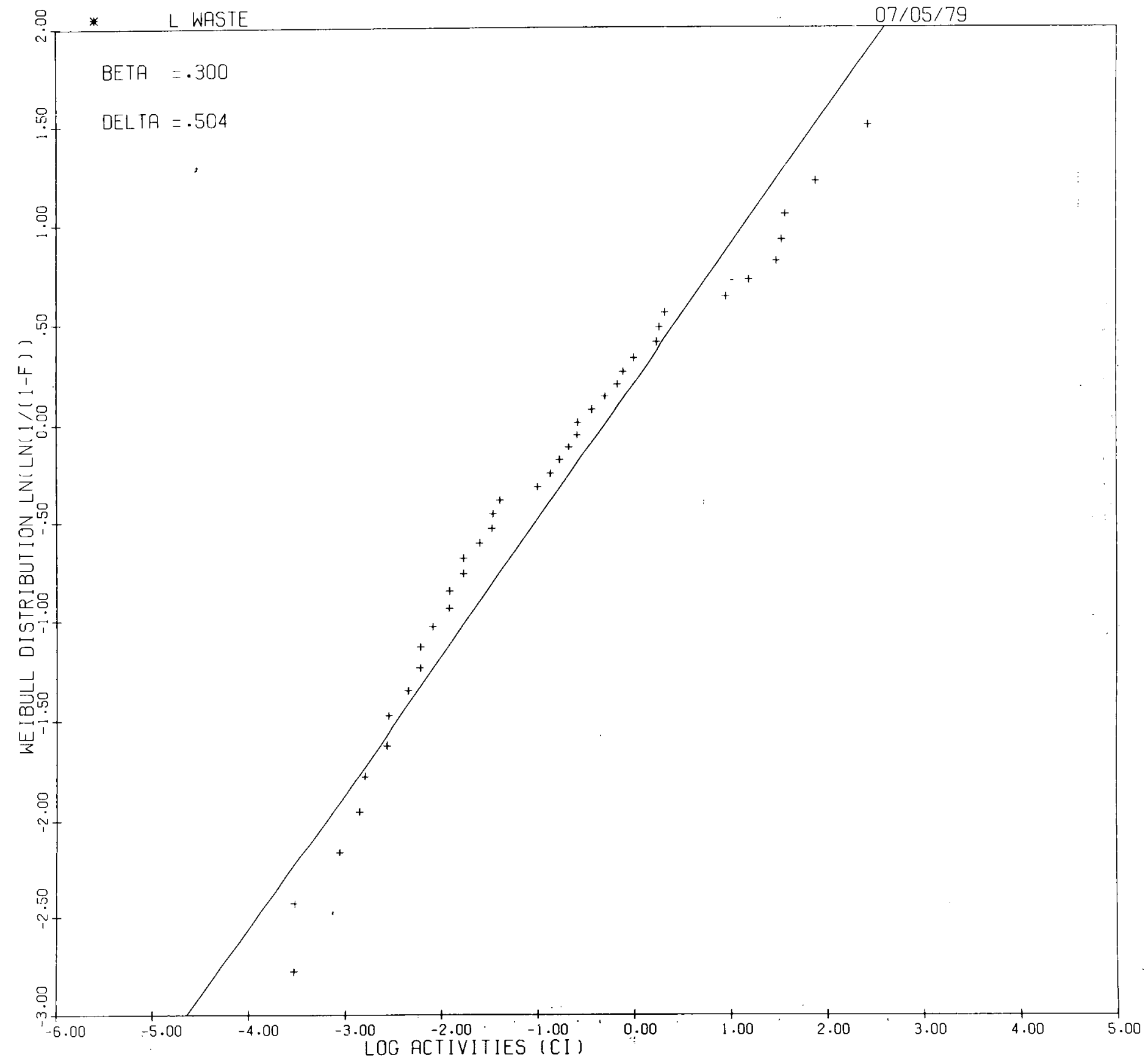

Figure A3a. Estimated CCF and the fitted Weibull CCF for the releases reproted in the LER file from liquid waste management system (a) Weibull probability paper (b) linear plot for the y axis 


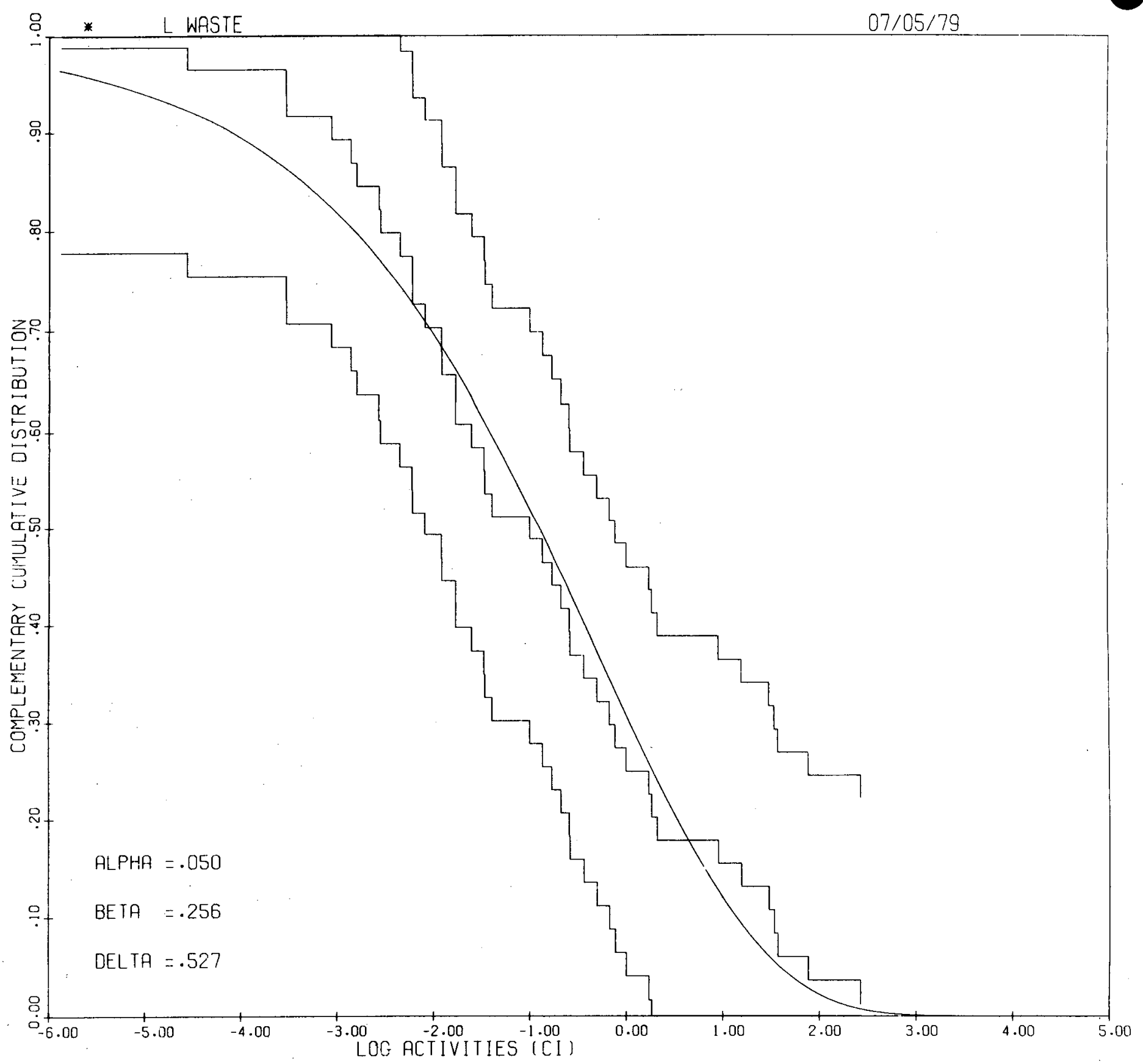

Figure A3b. Estimated CCF and the fitted Weibu11 CCF for the releases reported in the LER file from liquid waste management system (a) Weibull probability paper (b) linear plot for the $y$ axis 


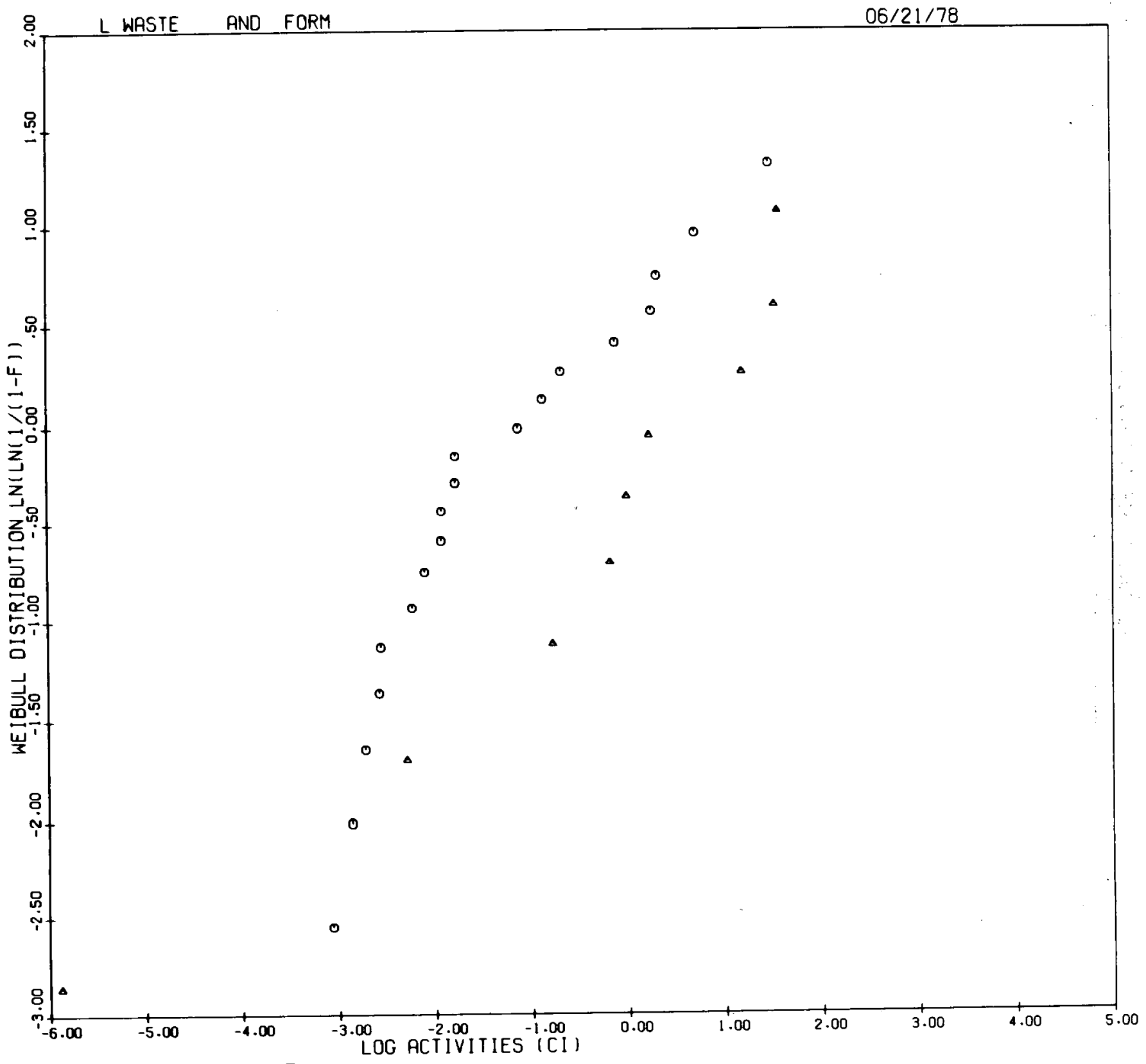

Figure A4a. Plots on Weibull probability paper of the releases broken down according to (a) form (b) cause code. In $4 a$ the symbols denote o liquid, gas. In $4 \mathrm{~b}$ the symbols denote 0 component failure, personnel error, + procedural error 


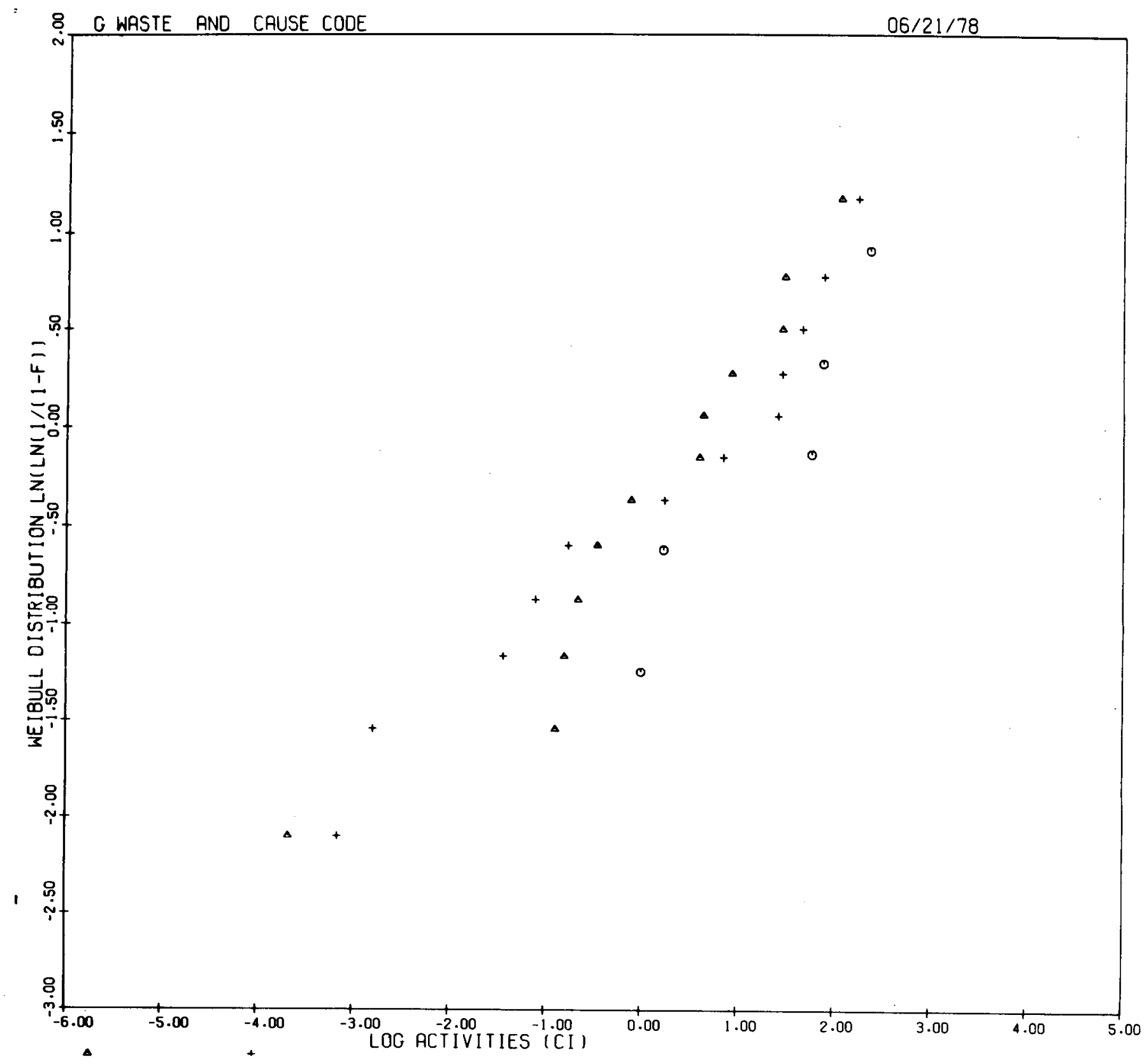

Figure A4b. Plots on Weibull probability paper of the releases broken down according to (a) form (b) cause code in $4 \mathrm{a}$ the symbols denote o liquid, gas. In $4 \mathrm{~b}$ the symbols denote 0 component failure, personnel error, + procedural error 
TABLE A1

STATISTICAL WEIBULL FIT

\section{Parameters}

no. $B \quad \underline{\text { range } B} \quad \delta \quad \underline{\text { range } \delta}$

Al1

$3.2 \quad 2.0-4.9$
Fit (\%significance level

Chi Mann

\section{Systems}

\begin{tabular}{|c|c|c|c|c|c|c|}
\hline Cont. & 14 & .28 & $.17-.37$ & 2.2 & $.34-15$ & \\
\hline cVCs & 30 & .26 & $.20-.32$ & 4.6 & $1.3-16$ & 13 \\
\hline $\begin{array}{l}\text { Gas Waste } \\
\text { Management }\end{array}$ & 48 & .35 & $.28-.41$ & 8.4 & $4 \cdot 1-18$ & 34 \\
\hline $\begin{array}{l}\text { Liquid Waste } \\
\text { Management }\end{array}$ & 42 & .26 & $.20-.30$ & .52 & $.18-1.6$ & 16 \\
\hline $\begin{array}{l}\text { Primary } \\
\text { Coolant }\end{array}$ & 23 & .40 & $.29-.51$ & 9.1 & $3.5-24$ & 6 \\
\hline $\begin{array}{l}\text { Secondary } \\
\text { Coolant }\end{array}$ & 17 & .20 & $.13-.25$ & 2.9 & $.28-32$ & 6 \\
\hline
\end{tabular}




\section{APPENDIX B}

CODE DESCRIPTION - WBFIT; A CODE TO

FIT A WEIBULL DISTRIBUTION TO DATA POINTS

A code, WBFIT, has been written to assist in the collation and statistical analysis of data on releases of fission products. The code plots a histogram of the observed data, calculates the parameters of a theoretical Weibull distribution and performs several statistical tests.

1. Data Plotting - Each set of data may be plotted separately, or, several different sets of data may be plotted on the same diagran. Two different plots are available, either or both may be selected.

a. Complementary Cumulative Distribution, F. This is defined as 1 minus the conventional cumulative distribution function so that $F(x)=$ (probability that $X \geq x$ ). Several estimators have been suggested for $F(x)$. The bias and mean squared error resulting from some commonly used estimators are given in Table 5.5, p. 216 of Reference 25. The estimator currently used in the code is

$$
\tilde{F}(i, n)=(i-1 / 2) / n
$$

The first plot is a histogram of $\tilde{F}(i, n)$ versus $\log \left(x_{j}\right)$, where $x_{j}$ is the $i^{\prime}$ th order statistic of a sample of size $n$.

b. Weibull Plot. Weibull probability paper is designed so that a Weibull 
distribution is a straight line. This is equivalent to plotting. . $\ln [\ln (1 / 1-F(x)))$ vs $\ln (x)$. The slope of the line is equal to $\beta$ and the intercept to $\beta \ln (\delta)$. The observed data, $x_{i}$, and estimate $F(i, n)$ are transformed as above and plotted, in order to assess visually the appropriateness of the hypothesis of Weibull distribution and also to display outliers. This plot is particularly useful to detect any major deviations from a Weibull form.

2. Plots of Theoretical Distributions - In either of the above two plots a curve corresponding to a theoretical distribution may be superimposed on the data.

3. Confidence Bands for the Distribution Function - For an unknown continous distribution function, the Kolmogorov-Smirnov statistic $D_{n}$ may be inverted to give a confidence band for the distribution function.

$$
P\left[\tilde{F}_{n}(x)-D_{n}(\alpha) \leq F(x) \leq \tilde{F}_{n}(x)+D_{n}(\alpha)\right]=1-\alpha
$$

Tables of the Kolmogorov distance $D_{n}(\alpha)$ have been taken from the Handbook of Probability \& Statistics 29 . The confidence band given by equation B2 may be plotted.

4. The Weibull Distribution - The two parameter Weibull distribution involves two parameters, $\beta$, a shape parameter, and $\delta$, a scale parameter. The probability density, $f(x)$, for the Weibull distribution is

$$
f(x)=\left(\frac{\beta}{\delta}\right)\left(\frac{x}{\delta}\right)^{\beta-1} \exp \left[-\left(\frac{x}{\delta}\right)^{\beta}\right]
$$

and the complementary cumulative distribution is 


$$
F(x)=\exp -(x / \delta)^{\beta}
$$

5. Estimation of the Parameters of the Weibull Distribution - Because of the versatility of the Weibull distribution, there is an extensive literature on various methods of estimation of the parameters and different applications of the distribution. Harter \& Moore(30) have shown that the maximum likelihood, best linear invariant and best linear unbiased estimators have nearly equal mean square error. Also it can be shown (24) that all these estimators are asymptotically efficient amd asymptotically normal and hence asymptotically tend to their respective Cramer-Rao bounds. Mann(31) has shown that moment estimators are less efficient. The calculation of linear estimators involves the extensive use of tables(24), which are available only for certain sample sizes. So the method of maximum likelihood has been selected in this code. The maximum likelihood equations for $\beta$ and $\delta$ are

$$
\begin{gathered}
{\left[\left(\begin{array}{lll}
n & \\
\Sigma_{1} & x_{i}^{\beta} & 1 n x_{i}
\end{array}\right) /\left(\begin{array}{ll}
n & \Sigma_{1}^{\beta} \\
1 & x_{i}^{\beta}
\end{array}\right)-(1 / \beta)\right]-\left(\Sigma 1 n x_{i}\right) / n=0} \\
\delta^{\beta}=\left(\Sigma_{1} x^{\beta}\right) / n
\end{gathered}
$$

Equation $B 5$ is an implicit equation for $\beta$ and is solved by the Newton-Raphson method. The iteration is stopped when the $(n+1)$ th approximation $\beta_{n+1}$ satisfies

$$
\left(\beta_{n+1}-\beta_{n}\right) / \beta_{n} \leq \varepsilon
$$


where accuracy $\varepsilon$ is specified in the input. $\hat{\delta}$ is then found from equation (B6). In order to start the iteration a first guess is required for $\beta$. This may be supplied as input, alternatively, if the approximate valve of $\beta$ is not known, the code will automatically generate an initial estimate of $\beta$. The code calculates this estimate by a procedure equivalent to using Weibull probability paper wherein $\beta$ is equal to the slope of the fitted straight line. In most of the cases considered less that 5 iterations are required for a fractional accuracy, $\varepsilon$, of .0001 .

The asymptotic covariance inatrix of $\hat{\delta} \& \hat{\beta}$ is 26

$$
\frac{1}{n}\left[\begin{array}{ll}
.608 \beta^{2} & .257 \delta \\
.257 \delta & 1.109 \delta^{2} / \beta^{2}
\end{array}\right]
$$

so $\hat{B} / \beta$ is asymptotically nornal with a mean 1 and variance $.608 / n \& \hat{B} \ln (\hat{\delta} / \delta)$ is asymptotically normal with mean 0 and variance $1.109 / n$. Thoman et al 26 have investigated the rate of convergence to the asymptotic distributions. For example the $5 \%$ confidence limits for $\beta$ based on the asymptotic expression agree with the true values within $1 \%$ for samples of size greater than 120 . Thoman et a) 26 have calculated the exact confidence intervals and the unbiasing factor for $\beta$ for small sample sizes using a Monte Carlo method. The tables given by these authors have been incorporated in the code. The code automatically corrects for the bias in the maximum likelihood estimator of $B$ and calculates the $5 \%$ \& $10 \%$, upper and lower confidence limits for $\beta$ and $\delta$. 6. Tests for Goodness of Fit to the Weibull Distribution

a. Graphical Test. The theoretical curves may be superimposed on plots of the observed data. This permits a visual inspection of the adequacy of the theoretical distribution. The second plot on "Weibull paper" 
would show up any large departures from linearity, and also is useful in detecting outliers.

n.

b. Kolmogorov-Smirnov Test. The Kolmogorov-Smirnov test is a classical test, which applies to testing the assumption that the data belongs to a completely specified distribution, i.e. one for which all the parameters as well as the form of the distribution are known. Analogs of the classical tests may be justified in certain circumstances(24) when the theoretical distribution involves estimated parameters. The statistic used in this test is

$$
D=\max _{i} \tilde{F}_{i}-\hat{F}\left(x_{i}\right)
$$

where $\tilde{F}_{j}$ is an estimate of the distribution function and $\hat{F}\left(x_{j}\right)$ is the value of the assumed distribution at the point $x_{j}$. The table of the critical values of $D$ at different significance levels is taken from the Handbook of Probability \& Statistics(29).

c. Chi Square Test. This test can be applied to a composite hypothesis, that is a distribution function which involves estimated parameters. However the data must be grouped so some infomation is lost. The sample space is divided into a number of intervals and the statistic

$$
W=\sum_{i}\left(0_{i}-e_{i}\right)^{2} / e_{i}
$$

is calculated where $0_{j}$ is the number of data points in the $j^{\prime}$ th internal and $e_{j}$ is the expected number predicted on the basis of the postulated distribution. The exact value for $W$ depends on the choice 
of intervals, a systematic procedure $(35)$ is to choose the intervals so that all the $e_{j}$ are equal. As a general rule the subdivision should be such that most $e_{i}>5$. The code uses a maximum number of intervals which is common with these two conditions. The distribution of $W$ is $x^{2}$ with 1-m-1 degrees of freedom where 1 is the number of intervals and $m$ the number of estimated parameters. A subroutine (GFIT) in the IMSL library is called to perform the Chi Square test. This test is best for larger sample sizes and is not applicable for samples smaller than 15.

d. Mann Test. Mann et al (27) have developed a goodness of fit test specific to the Weibull distribution. For brevity, we shall here refer to this as the Mann test. Mann et al(27) have shown that the Mann test is more powerful than the analog of the Kolmogorov-Smirnov test and several other tests, against the hypothes is of a log normal or three parameter Weibull distribution. The test statistic $S$ is

$$
S=\left(\sum_{i=[n / 2]+1}^{n-1} \quad 1_{i}\right)\left(\sum_{1}^{n-1} 1_{i}\right)
$$

where

$$
\begin{aligned}
1_{i}= & \left(T_{i+1}-T_{i}\right) /\left(E\left(z_{i+1}\right)-E\left(z_{i}\right)\right) \\
& T_{i}=\ln x_{1} \\
z_{i}= & \beta\left(\ln x_{i}-\ln \delta\right)
\end{aligned}
$$

and $x_{j}$ is the $i$ 'th order statistic. The asymptotic distribution of $S$ is a beta distribution with parameters $[(n-1) / 2]$ and $[n / 2]$. Mann et al (27) have calculated the percentiles of the distribution of $S$ using 
a Monte Carlo method. The computed values agree to $10 \%$ with those based on a beta distribution for $n>16$. The code uses tables of the distribution for $S$ and tables for $\left(E\left(Z_{i+1}\right)-E\left(Z_{i}\right)\right)$ to calculate a significance level for the theoretical distribution. 
APPENDIX C

\section{WASTE GAS DECAY TANK INVENTORY}

As indicated in the Surry SAR, the combined volume of the two Surry waste gas decay tanks is sized to process gas from the estimated annual average letdown flow of $17 \mathrm{gpm}$ to the Boron Recovery System gas strippers. The average letdown flow is based on simultaneous operation of the two reactor units. The average gas stripping rate is proportional to the average letdown flow rate. This average flow rate depends on the plan of operation for the reactor plant. As indicated in Section 9.2 of the Surry SAR, the Boron Recovery System can accommodate letdown flow due to daily load following and weekend load reductions on both units to nearly the end of core life with the daily load follow cycle basis consisting of $12 \mathrm{hr}$. at full power, a uniform 3 hr. ramp reduction to 50 percent power, $6 \mathrm{hr}$. at 50 percent power, and a uniform $3 \mathrm{hr}$. ramp increase to full power. On this basis, the average annual letdown flow for the two units is $17 \mathrm{gpm}$. The gas decay tanks are sized.so that a $17 \mathrm{gpm}$ letdown rate with the recombiner not operating gives an average yas holdup time equivalent to approximately 5 half-lives of Xe-133 or 30 days.

The volumetric flow of gas stripped from the primary coolant can be estimated as follows. At a $17 \mathrm{gpm}(64.35 \mathrm{1} / \mathrm{min})$ letdown flow rate to the Boron Recovery System, assuming a water density of $1 \mathrm{~g} / \mathrm{cc}$, the mass flow rate is 64.4 $\mathrm{kg} / \mathrm{min}$. The concentration of hydrogen in the primary coolant is assumed to be $35 \mathrm{cc} / \mathrm{kg}$ and is 90 percent by volume of the gas in the coolant. If we assume all the gases are stripped in the gas strippers, then the gas stripping rate, QSR, is given by

$$
\begin{aligned}
Q_{S R}= & W_{L F} \times C_{H} / f_{H} \\
& -157-
\end{aligned}
$$


where

$$
\begin{aligned}
& W_{L F}=\text { letdown mass flow rate, } \mathrm{kg} / \mathrm{min} \\
& C_{H}=\text { hydrogen concentration in primary coolant, cc/ } \mathrm{kg} \\
& f_{H}=\text { hydrogen fraction of gases in primary coolant. }
\end{aligned}
$$

For the parameters given above, the average annual gas stripping rate is given as

$$
Q_{S R}=2500 \mathrm{cc} / \mathrm{min}=0.088 \mathrm{scfm}
$$

In Table 11.2.1 of the Surry SAR, the waste gas decay tank volume is given as $434 \mathrm{ft}^{3}$ and the operating pressure is given as $115 \mathrm{psig}$. Assuming the waste gas is initially at atmospheric pressure, the time to fill a tank is given by

$$
t_{F}=P_{O P} V_{D T} / 1440 P_{A T M} Q_{S R}
$$

where

$$
\begin{aligned}
t_{F} & =\text { time to fill one decay tank, days } \\
P_{O P} & =\text { tank operating pressure, } 130 \text { psia } \\
V_{D T} & =\text { gas decay tank volume, } 434 \mathrm{ft}^{3} \\
P_{A T M} & =\text { atmospheric pressure, } 15 \text { psia, and } \\
Q_{S R} & \text { is defined above. }
\end{aligned}
$$

Using the above defined parameters, the time to fill one tank, assuming the recombiners are not operating, is about 30 days.

The Surry SAR states that if the gas recombiners are operating, the gas volume to the waste gas decay tanks is reduced 90 percent by the recombiners. In this case, the number of days to fill the waste gas decay tanks would increase by a factor of 10 to about 300 days.

The buildup of activity in the waste gas decay tanks can be estimated as follows. Assume that waste gas is fed to the waste gas decay tank at a 
constant rate, $Q$, with constant concentrations of various radionuclides, $a_{j} \cdot$ In the tank each nuclide decays at a rate proportional to its decay constant, $\lambda_{i}$. The following differential equation describes the buildup of activity, $A_{j}$, in the tank:

$$
\frac{d A_{i}}{d t}=Q a_{i}-\lambda A_{i}
$$

where

$$
A_{j}(0)=0
$$

Solving for $A_{j}(t)$, the tank activity at any time $t$,

$$
A_{i}(t)=\frac{Q a_{i}}{i}\left(1-e^{\left.-\lambda_{i} t\right)}\right.
$$

The equilibrium activity is reached when $A_{j}$ does not change with time and is given by

$$
A_{i}=Q a_{i} / \lambda_{i}
$$

In using this approach, we have neglected the buildup of activity due to daughter isotopes.

Table El provides the equilibrium activity in the waste gas decay tank for various noble gas and iodine isotopes for a clad defect level of 0.12 percent. The equilibrium primary coolant activity concentrations are based on values estimated from the PWR-GALE report. The waste gas activities are based on a $17 \mathrm{gpm}$ average annual letdown flow to the gas strippers where 100 percent of the noble gases and 0.1 percent of the iodines are removed from the primary coolant. The recombiners are assumed to be operating. Thus, the feed cycle is 300 days in duration.

During the course of reactor operations, radioactive gases in the vapor space of the Volume Control Tank (VCT) and gases resulting from the 
degasification of the primary coolant prior to cold or refueling shutdowns would also be directed to the waste gas decay tanks for holdup prior to release. The inventory of gases in the prinlary coolant which would be directed to the waste gas decay tanks in the event of a cold shutdown can be estimated from the equilibrium coolant activities given in Table Cl and the mass of the primary coolant, estimated to be $1.78 \times 10^{8} \mathrm{~g}$. The next-to-the-last column of Table C1 tabulates the noble gas and iodine activity which may be directed to the waste yas decay tanks after degasification of the primary coolant. This tabulation assumes 100 percent of the noble gases and 0.1 percent of the iodines are degasified. The radionuclide inventories within the vapor space of the VCT have been estimated in Appendix D. These are tabulated in the last column of Table Cl.

Since the primary coolant is degasified and the vapor space of the VCT vented on cold or refueling shutdowns, it was of interest to estimate the number of cold shutdowns per reactor-year. In Appendix $V$ of the Reactor Safety Study(1) it was estimated that there were cold shutdowns, but three of the ten were orderly, slow shutdowns for maintenance, leaks, and so forth. These three could be considered cold shutdowns. An EPRI report(32) discussing sources of radioiodine at pressurized water 'reactors (PWR) provided shutdown information for three PWR's. Based on this information, the number of cold shutdowns was estimated to be three per reactor year, or for the two Surry reactors, a total of six cold shutdowns per year. It is assumed that two of these shutdowns are refueling shutdowns which would occur at a known time. The other four shutdowns could be assumed to occur randomly within the operating cycles of the two reactors. 
In the event of a cold shutdown, the waste gas decay tank activity would be increased by the gases vented from the VCT vapor space and the gases resulting from primary coolant degasification. For the noble gas of primary interest, Xe-133, the tank activity would be increased by about 35 percent by the activity from these sources. We think this variation would be within the uncertainty of the activity within the waste gas decay tank and would ignore it in estimating the waste gas decay tank radionuclide inventory.

For the majority of the radionuclides under consideration here, equilibrium is established in the waste gas decay tank in a time period on the order of several days. Thus the radionuclide concentration in the decay tank can be assumed to be directly proportional to the instantaneous clad defect level in the core. Equation C.7 expresses this relationship.

$$
A_{j}=B_{i} \times f_{c d}
$$

where

$$
\begin{aligned}
B_{i} & =\text { constant of proportionality } \\
f_{C d} & =\text { percent of fuel cladding defected. }
\end{aligned}
$$

The values of $B_{j}$ are presented in Table $C 2$. Since the probability density function for clad defect level, god, is known, the density function, $g_{j}\left(A_{j}\right)$, for the concentration of each radionuclide in the waste gas decay tank is:

$$
g_{i}\left(A_{i}\right)=\frac{1}{B_{i}} \quad g_{c d}\left(\frac{A_{i}}{B_{i}}\right)
$$

For the longer lived radionuclides such as $\mathrm{kr}-85$, a statistical treatment of the inventories would be more difficult because a relationship has not been derived between defect level and time in a cycle. 
Since the activity of Xe-133 in the Waste Gas Decay Tank is an order of magnitude greater than the maximum activity of $k r-85$ and because the health effects per curie of Xe-133 are greater than for $\mathrm{Kr}-85$, it should be acceptable to limit consideration to $\mathrm{Xe}-133$ in the analysis of an accident in the Waste Gas Decay Tank. The more difficult problem of deriving a density function for $\mathrm{Kr}-85$ inventory does not have to be resolved, therefore, another argument for ignoring the release of $\mathrm{Kr}-85$ in this accident is that it would all be eventually released anyway. 
APPENDIX D

VOLUME CONTROL TANK VAPOR SPACE ACTIVITY

\section{D.1 Source Term Calculations}

The Volume Control Tank (VCT) is part of the chemical and volume control system (CVCS) in the Surry Plant. The CVCS provides a variety of functions to the plant including the following:

1. Injection of negative reactivity in the form of boric acid solution

2. Chemical additions for corrosion control

3. Reactor coolant cleanup and degasification

4. Addition of Reactor Coolant System (RCS) makeup water

5. Seal water injection to the reactor coolant pump seals

6. High pressure flow to the Safety Injection System (SIS)

7. RCS filling

8. RCS draining to the Primary Drain System (PDS) is accomplished through the excess letdown flow path of the CVCS.

Figure D1 is a graphic representation of the Surry Volume Control Tank.

The vapor space of the VCT, which contains primarily hydrogen and water vapor, is automatically supplied hydrogen as detemined by pressure control. The hydrogen in the VCT is the supply source for the reactor coolant. Fission gases are periodically renoved from the system by venting the VCT to the vent and drain system (VDS) or by diverting the letdown stream to the gas stripper in the Boron Recovery Systen (BRS) prior to a cold or refueling shutdown. The following model is used to estimate the activity of noble gases in the vapor 


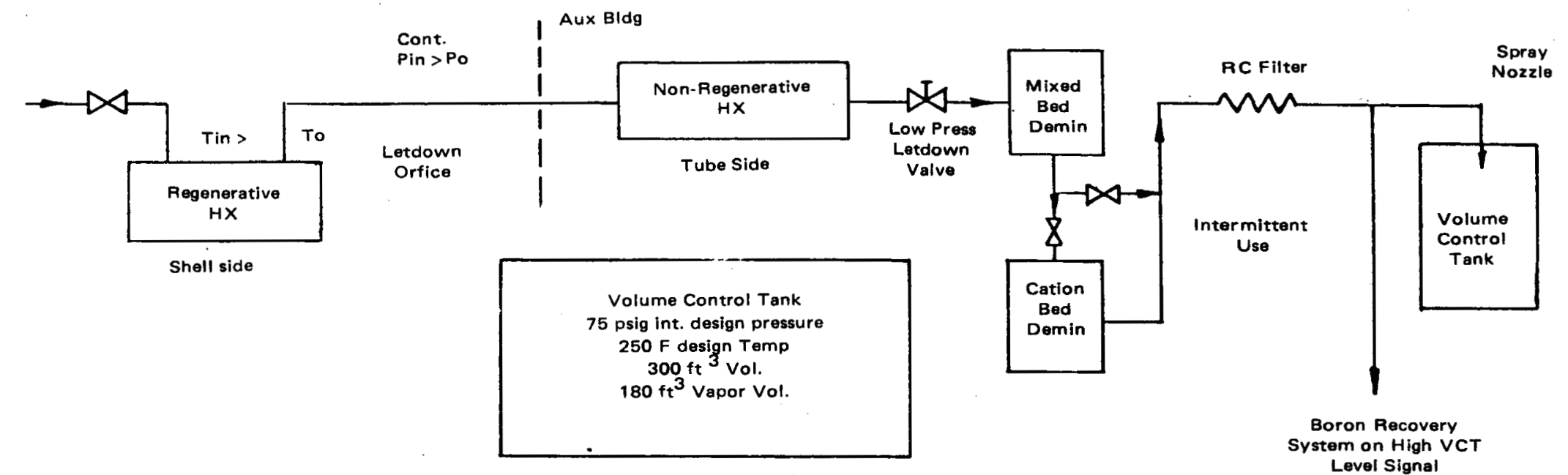

Letdown Flow Periodic Venting
to Vent \& Drain System

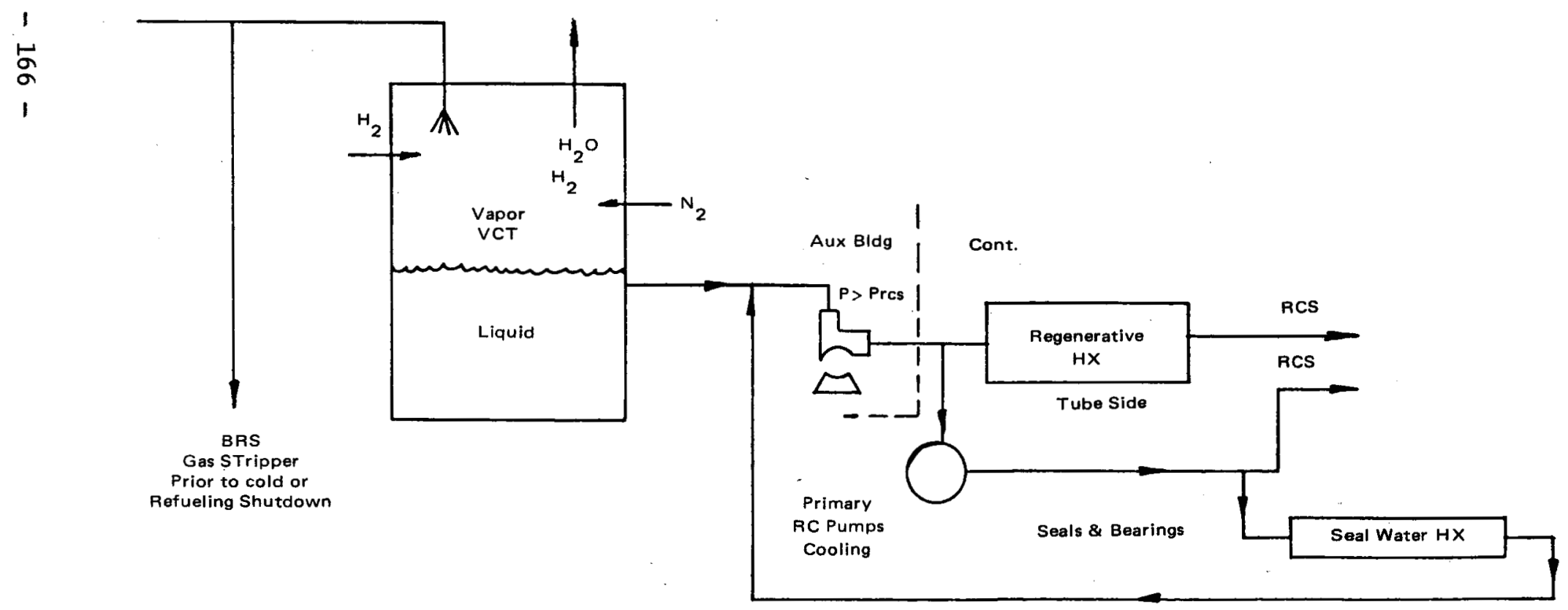

Figure Dl. Schematic of the chemical and volume control system of Surry 
space of the volume control tank. Referring to Figure D2, a liquid stream of volume flow $Q$ with a concentration of species $i$ given by $C_{j}$ is sprayed into the volume control tank. The stripping fraction for the spray process is given by $\alpha$. A liquid steam of volume flow $Q$ leaves the volume control tank. The rate of change of activity of species $i$ in the gas space and liquid space of the volume control tank is governed by the following equations:

$$
\begin{aligned}
& Q C_{i} \alpha-\lambda_{i} G_{i}-T_{G W}=\frac{d G_{i}}{d t} \\
& Q C_{j}(1-\alpha)-\lambda_{i} L_{i}+T_{G W}-Q L_{i} / V=\frac{d L_{i}}{d t}
\end{aligned}
$$

where

$$
\begin{aligned}
Q & =\text { liquid volume flow } \\
C_{j} & =\text { concentration of species } i \text { in incoming volune flow } \\
\lambda_{i} & =\text { decay constant for species } i \\
G_{i} & =\text { activity of species } i \text { in the gas space } \\
L_{i} & =\text { activity of species } i \text { in the liquid space } \\
V & =\text { volume of the liquid space in the volume control tank } \\
T_{G W} & =\text { rate of transfer of activity from the vapor to the liquid } \\
\alpha & =\text { stripping fraction for the spray process. }
\end{aligned}
$$

Summing Equations D.1 and D.2, the following equation is obtained.

$$
Q C_{i}-\lambda_{i} A_{i}-Q L_{i} / V=\frac{d A_{i}}{d t}
$$

where

$$
A_{j}=G_{j}+L_{i}
$$




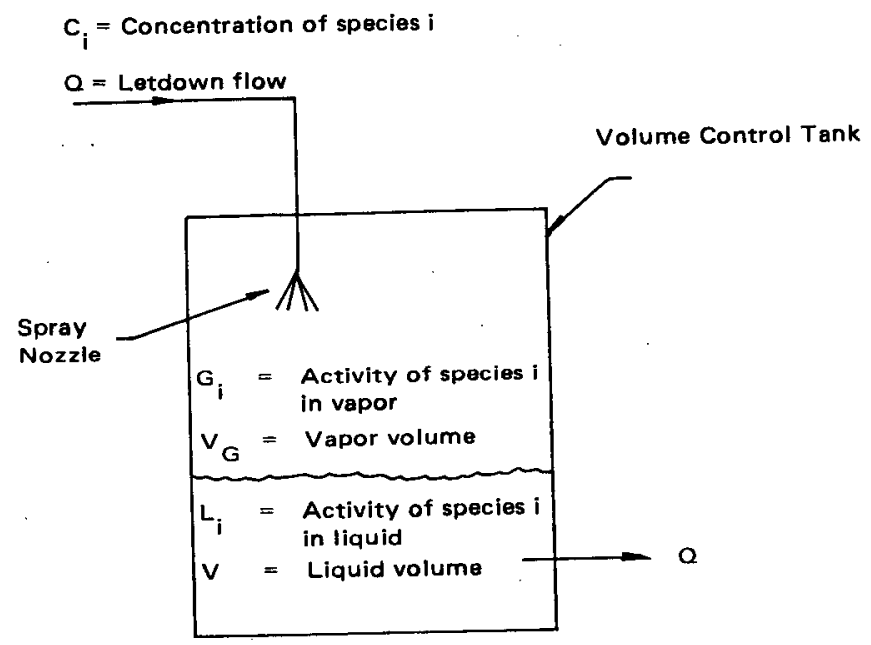

Figure D2. Idealized schematic of stripping process in volume control tank 
If we assume that species $i$ in the liquid and vapor spaces are in equilibrium, then

$$
G_{j} / V_{G}=k_{i} L_{i} / V
$$

where

$V_{G}=$ volume of the vapor space in the volurne control tank

$k_{j}=$ partition coefficient for species $i$ between the liquid and vapor.

Combining Equations D.3, D.4, and D.5 to eliminate $A_{j}$ and $L_{j}$ from Equation D.3, we get

$$
\begin{gathered}
\left.\left(1+V / k_{i} V_{G}\right) \frac{d G_{i}}{d t}=Q C_{i}-\lambda_{i}\left(1+V / k_{i} V / V\right)\right) G_{i} \\
-\left(Q\left(1+V / k_{i} V_{G}\right) / V\left(1+k_{j} V_{G} / V\right)\right) G_{i}
\end{gathered}
$$

or

$$
\frac{d G_{i}}{d t}=Q C_{i} /\left(1+V / k_{i} V_{G}\right)-\lambda_{i} G_{i}-\left(Q / V\left(1+k_{i} V_{G} / V\right)\right) G_{i}
$$

Assuming that $G_{i}(0)=0$, the solution to Equation $D .7$ is

$$
G_{i}=\left(Q C_{i} /\left(1+V / k_{i} V_{G}\right) \lambda_{i}^{*}\right)\left(1-e^{-\lambda_{i}^{*} t}\right)
$$

where

$$
\lambda_{i}^{*}=\lambda_{i}+Q / V\left(1+k_{i} V_{G} / V\right)
$$

This model will be used to estimate the maximum noble gas activities in the Surry Plant volume control tank vapor space. In order to carry out the calculations, the partition coefficients, $k_{j}$, for the noble gases of interest, 
krypton and xenon, must be estimated. This estimate is carried out in Section D.2. Table D1 presents the parameters used in determining the maximum noble gas activities in the volume control tank vapor space. Table D2 presents the calculated activities and, for comparison purposes, presents the maximum activities as detemined from the vapor space noble gas activity concentrations given in Surry SAR Table 9.1 - 6 .

We believe that the equilibrium inventories given in Table $D 2$ which are based on the PWR Gale report(7) modified fission product activities represent the best estimate of the activity in the vapor space of the Volume Control Tank. In lieu of actual operating data, we would use this inventory as the best estimate of activity which might be released from the VCT vapor space.

Because equilibrium is established in the vapor space of the volume control tank within a day for all radionuclides, the concentration in the vapor space can be assumed to be directly proportional to the instantaneous clad defect level in the core.

$$
G_{j}=B_{j} \times f_{c d}
$$

where $B_{j}=$ constant of proportionality

$f_{c d}=$ percent of fuel cladding defected.

Values of $B_{j}$ are presented in Table D3.

Since the probability density function for clad defect level, $g_{c d}$, is known, the density function, $g_{j}\left(G_{j}\right)$, for the concentration of each radionuclide in the vapor space of the volume control tank is:

$$
g_{j}\left(G_{i}\right)=\frac{1}{B_{i}} g_{C d}\left(\frac{G_{i}}{B_{i}}\right)
$$




\section{TABLE D1}

PARAMETERS USED IN CALCULATING MAXIMUM NOBLE GAS ACTIVITIES IN VOLUME-CONTROL TANK VAPOR SPACE

$\begin{array}{rlrl}Q & =120 \mathrm{gpm}^{*}=16.04 \mathrm{cfm} \text { (maximum) } & & \text { Surry SAR Table } 9.1-2 \\ V & =120 \mathrm{ft}^{3} & & \text { Surry SAR Table } 9.1-5 \\ V_{g} & =180 \mathrm{ft}^{3} & & \text { Surry SAR Table 9.1-5 } \\ k_{k r} & =24.0 & & \text { Appendix A } \\ k_{\mathrm{xe}}=13.1 & & \text { Appendix A }\end{array}$

*The volumetric flow rate is based on water at $130 \mathrm{~F}$ and 2365 psia $\left(p=621 b_{m} / \mathrm{ft}^{3}\right)$. 
TABLE D2

VOLUME CONTROL TANK VAPOR SPACE NOBLE GAS ACTIVITY INVENTORY

\begin{tabular}{|c|c|c|c|c|c|c|}
\hline Isotope & $\begin{array}{c}\text { Decay } \\
\text { Constant } \lambda_{i} \\
(\min )^{-1}\end{array}$ & $\begin{array}{c}\text { Prinlary } \\
\text { Coolant } \\
\text { Concentration } \\
\mu \mathrm{C} i / \mathrm{CC}\end{array}$ & $\begin{array}{c}\text { Vapor Space } \\
\text { Inventory } \\
\mathrm{Ci} \\
\end{array}$ & $\begin{array}{c}\text { SAR } \\
\text { Vapor Space } \\
\text { Inventory } \\
\mathrm{Ci} \\
\end{array}$ & $\begin{array}{c}\text { PWR-GALE } \\
\text { Modified Primary } \\
\text { Coolant } \\
\text { Concentration } \\
\text { Ci/CC } \\
\end{array}$ & $\begin{array}{c}\text { PWR-GALE } \\
\text { Modi fied } \\
\text { Vapor Space } \\
\text { Inventory } \\
\mu \mathrm{Ci} \\
\end{array}$ \\
\hline $\mathrm{Kr}-85$ & $1.23(-7)$ & 2.42 (peak) & NA & 9.4 & 0.01 & 1.6 \\
\hline$k r-85 m$ & $2.63(-3)$ & 1.14 & 109.1 & 185 & 0.081 & 5.5 \\
\hline $\mathrm{Kr}-87$ & $9.12(-3)$ & 0.78 & 36.6 & 37.2 & 0.046 & 1.3 \\
\hline $\mathrm{Kr}-88$ & $4.14(-3)$ & 2.81 & 216.3 & 196.7 & 0.15 & 7.4 \\
\hline$x e-133$ & $9.13(-5)$ & 188 & 16723 & 15393 & 5.5 & 487 \\
\hline$x e-133 m$ & $2.13(-4)$ & 1.87 & 162.8 & 167.2 & 0.10 & 8.1 \\
\hline$x e-135$ & $1.26(-3)$ & 5.2 & 392.5 & 345.6 & 0.24 & 15.9 \\
\hline$x e-135 m$ & $4.42(-2)$ & 0.13 & 1.5 & 1.1 & 0.010 & 0.064 \\
\hline$X e-138$ & $4.88(-2)$ & 0.35 & 3.7 & 3.7 & 0.034 & 0.19 \\
\hline
\end{tabular}

(1) Taken from Surry SAR Table 9.1-5.

(2). Calculated using the model presented here.

(3) Obtained by multiplying vapor space concentrations given in Surry SAR Table 9.1-6 by the volume of the vapor space in the volume control tank.

(4) As detemined in Appendix B.

(5) Calculated using model presented here with nomal letdown flow $(60 \mathrm{gPm})$ and the PWR-GALE modified primary coolant concentrations given here. 


\section{TABLE D3}

CONSTANTS OF PROPORTIONALITY FOR USE IN DETERMINING THE

PROBABILITY DENSITY FUNCTION FOR THE RADIONUCLIDES

IN THE VAPOR SPACE OF THE VOLUME CONTROL TANK

\begin{tabular}{lcc} 
Isotope & $\begin{array}{c}\text { Volume Control Tank } \\
\text { Vapor Space } \\
\text { Activities, } G_{i} \mathrm{Ci}\end{array}$ & $\begin{array}{c}\text { Constant of } \\
\text { Proportionality } \\
\text { B Ci/\% Failed Fuel }\end{array}$ \\
\hline $\mathrm{Kr}-85$ & 1.6 & 13.3 \\
$\mathrm{Kr}-85 \mathrm{~m}$ & 5.5 & 45.8 \\
$\mathrm{Kr}-87$ & 1.3 & 10.8 \\
$\mathrm{Kr}-88$ & 7.4 & 61.7 \\
$\mathrm{Xe}-133$ & 487 & 4058.3 \\
$\mathrm{Xe}-133 \mathrm{~m}$ & 8.1 & 67.5 \\
$\mathrm{Xe}-135$ & 15.9 & 132.5 \\
$\mathrm{Xe}-135 \mathrm{~m}$ & 0.064 & 0.53 \\
$\mathrm{Xe}-138$ & 0.19 & 1.6
\end{tabular}




\section{D.2 Estimation of Patition Coefficients for Krypton and Xenon}

From the Handbook of Chemistry and Physics, (33) Henry's Law Constants, $K_{j}$, for krypton and xenon at $55 \mathrm{C}$ are estimated to be $2.55 \times 10^{7} \mathrm{~mm}$ $\mathrm{Hg} /(($ mole $\mathrm{Kr}) /($ mole water $))$ and $1.39 \times 10^{7} \mathrm{~mm} \mathrm{Hg} /(($ mole $\mathrm{Xe}) /($ mole water $)$ ) respectively. At $55 \mathrm{C}$, one mole of gas occupies 25.1 liters at one atmosphere. The partition coefficient, $k_{i}$, is given by

$$
k_{i}=k_{i} \frac{\mathrm{mm} \mathrm{Hg}}{\text { (mole gas/mole water) }} \times \frac{\mathrm{atm}}{760 \mathrm{~mm} \mathrm{Hg}} \times \frac{\text { mole gas }}{25.1 \mathrm{~L}-\mathrm{atm}}
$$

$k_{i}=9.44 \times 10^{-7} k_{i}$

$$
\times \frac{\mathrm{L}}{1000 \mathrm{cc}} \times \frac{18 \mathrm{~g}}{\text { mole water }} \times \frac{\mathrm{cc}}{1 \mathrm{~g}}
$$

For krypton, $k_{k r}=24.0$

for xenon, $k_{X e}=13.1$

\section{D.3 Primary Coolant Noble Gas Concentrations}

The PWR-GALE ( 7 ) report provides a method for estimating the primary coolant noble gas concentrations, taking into account Surry plant conditions. The report gives the primary coolant noble gas concentrations for a reference PWR with U-tube steam generators as given in Table D 4. Since design parameters for the Surry plant are outside the ranges specified in the PWR-GALE report, the concentrations must be adjusted using the Surry design parameters. The adjustment factor used to accomplish this for the noble gases is given by 
TABLE D4

PWR GALE REPORT REACTOR COOLANT CONCENTRATIONS

(2)

Isotope $\begin{gathered}\operatorname{Activity}(1) \\ \mu \mathrm{Ci} / \mathrm{g}\end{gathered} \lambda_{i}(\mathrm{hr})^{-1}$

(3)

$0.15 \quad 7.37 \mathrm{E}-6 \quad .089$

0.11

0.1575

0.994

0.062

0.046

$\mathrm{kr} 87$

0.06

0.5472

1.04

1.02

0.20

0.15

Xe 133

18

$5.48 \mathrm{E}-3$

0.414

7.5

5.5

Xe 133m

0.22

0.0128

0.613

0.13

0.10

Xe 135

0.35

0.0756

0.935

0.33

0.24

Xe $135 \mathrm{~m}$

0.013

2.649

1.052

0.014

0.010

Xe 138

$0.044 \quad 2.929$

1.053

0.046

0.034
0.11

(5)

Activity $\mu \mathrm{Ci} / \mathrm{Cc})$

0.01

.081

Activity Modified 


$$
f_{i}=\frac{162}{W P} \frac{\left(0.0009+\lambda_{i}\right)}{\left(R+\lambda_{i}\right.} .
$$

where

$f_{j}=$ adjustment factor for the $i$-th isotope

$P=$ Reactor thermal power, MWt

$W B=$ Weight of water in the reactor coolant system excluding pressurizer, $1 b_{m}$

$\lambda=$ Decay constant of the $i$-th isotope, $(h r)^{-1}$

and

$$
R=(F B+F D \quad Y) / W P,
$$

where

$\mathrm{FB}=$ Reactor coolant letdown flow (yearly average for boron control), $1 b_{m} / h r$

$F D=$ Reactor coolant letdown flow (purification), $1 \mathrm{~b}_{\mathrm{m}} / \mathrm{hr}$

$Y=$ Ratio of total anount of noble gases routed to gaseous radwaste from the purification system to the total amount routed from the primary coolant to the purification system (not including the boron recovery system).

For the Surry Plant,

$W P=3.91 \times 10^{5} \mathrm{I} \mathrm{b}_{\mathrm{m}}$ at primary system pressure and temperature

$\mathrm{FB}=4.23 \times 10^{3} 1 \mathrm{~b}_{\mathrm{m}} \mathrm{lb}(8.5 \mathrm{gpm})$

$F D=2.98 \times 10^{4} 1 \mathrm{~b} / \mathrm{hr}(60 \mathrm{gpm}) \quad$ Table 9.1-4-Surry SAR

$Y=0$

$P=2546$ MWt

Table 9.1-4-Surry SAR 
For the Surry design conditions,

$$
R=0.0108,
$$

and

$$
f_{i}=1.055 \quad \frac{0.0009+\lambda_{i}}{0.0108+\lambda_{i}}
$$

The concentrations of each of the noble gases are then multiplied by the appropriate adjustment factor, $f_{j}$. Table $D 4$ gives the resulting noble gas concentrations based on Surry conditions. In Table D4 concentrations are also given in the units $(\mu \mathrm{Ci} / \mathrm{cc}$ ) which allow a direct comparison to the concentrations presented in Table D2 of this write-up. 
APPENDIX E

\section{ESTIMATED ACTIVITY LEVELS IN VARIOUS}

WASTE DISPOSAL SYSTEM TANKS

Radioactive liquid wastes from various sources are processed in the liquid waste disposal system (LWDS). In the Surry Plant, the LWDS is common to both units and designed to accommodate wastes from both units simultaneously. In carrying out its functions of separating, treating, and disposing of liquid wastes, the LWDS makes use of the following basic processes:

1) Filtration for removal of particulate matter

2) Evaporation for concentration of radioactive constituents into liquid and vapor phase separation

3) Demineralization for removal of dissolved material

4) Retention for promoting radioactive decay

5) Dilution for concentration reduction.

Liquid wastes originate in the Reactor Coolant System, the auxiliary and emergency systems, the Waste Disposal System, and as a result of operational and maintenance procedures. These wastes can enter the LWDS directly from their source or via the Vent and Drain System (VDS). The VDS classifies process liquids either for re-use or disposal. As a result, only a small fraction of the VDS throughput is directed to the LWDS.

A series of three holding tank subsystems make up the waste collection portion of the LWDS in the Surry Plant with the influents from the VDS discharged to the various holding tanks according to their activity levels. These tanks include the high level and low level waste drain tanks and the contaminated drain tanks. The high level waste drain tanks receive liquid 
waste from a variety of sources including the Boron Recovery System (BRS), boron recovery tank sumps, laboratory drains, Chemical and Volume Control System, waste disposal evaporation test tanks, contaminated drains tanks, and if necessary, the low level waste drain tanks. The low level waste drain tanks can receive liquid waste from the BRS test tanks, the VDS, the decontamination tanks, and if of sufficiently low activity, the high level waste decay tanks. The contaminated drain tanks receive liquid wastes from the laundry drains and the PCA shower and lavatory drains.

As indicated in the Surry SAR, the high level waste drain tank contents, which may have activity levels on the order of $10^{-1} \mu \mathrm{Ci} / \mathrm{cc}$, are processed by evaporation in the waste disposal evaporator to reduce the activity levels in the evaporator distillate to no greater than $10^{-4} \mu \mathrm{Ci} / \mathrm{cc}$. The distillate is pumped to the Waste Disposal Test Tanks (WDTT) for sampling to determine the resultant radioactivity level and the chemical composition. If still contaminated, the test tank contents may be further purified by circulating the contents through a mixed-bed demineralizer and filter or by recirculating the contents through the evaporator. If the tank contents are below some specified maximum activity level, they are discharged directly through the liquid waste radioactivity monitoring and flow control stations. If the activity level of the contents of the high level waste drain tanks is such that evaporation is not required, these contents can be transferred to the low level waste drain tanks via a drain line under administrative control. Contents of both the low level waste drain tanks and the contaminated drains tanks are both filtered and monitored prior to discharge to the circulating water discharge canal. The contents of these tanks can be pumped to the high level 
waste drain tanks for evaporation if the liquid activity level exceeds about

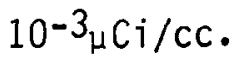

Sources of PWR liquid wastes, along with their estimated annual flow rates and activity levels, are identified in the Surry SAR and in the PWR-GALE Report(7). Tables E1 and E2 tabulate the information available there concerning these liquid waste sources.

In order to estimate the activity levels in the Waste Disposal Test Tanks, we will identify the possible influents to these tanks and the processing they have received in the Liquid Waste Disposal System. As indicated earlier, the WDTT's collect distillate from the waste disposal evaporator and hold this. liquid to determine if further processing is needed or release to the environment is possible. Liquid entering the waste disposal evaporator is pumped from the High Level Waste Drain Tanks. These tanks can receive effluents from a variety of sources, including the following:

1) The Primary Drain Tank (PDT)

2) The Boron Recovery Tank (BRT) sump

3) Liquid from the Catalytic Recombiner Moisture Separator in the Gas Waste Disposal System

4) Waste Disposal Evaporator bottoms

5) Waste Disposal Evaporator distillate

6) Contaminated Drains Tanks

7) Laboratory Drains

8) Low Level Waste Drain Tanks

In order to identify possible sources of liquid waste for the High Level Waste Drain Tanks, we have examined, in addition to the Surry SAR, the SAR's for Salem, NicGuire, Ginna, Oconee, Fort Calhoun, and Sequoyah. Liquid waste 
TABLE E1

PWR LIQUID WASTES (TWO UNITS) (1)

Waste Source

Laundry Drains

Sampling Sinks (Turbine and Aux. Bldg.)

\section{Boron Recovery}

System Letdown

Spent Resin Flush

Laboratory Wastes

Reactor Coolant

System Leakage
Flow Rate(2)

gpd

855

315

4210

110

140

11
1.0

1.0

$2.852(-3)$

Fraction of Primary Coolant Activity

$1.71(-6)$

$14.5(3)$

1.0

(1) Based on information presented in Figure 11A-1, Surry SAR

(2) Assuming 292 days/year operation

(3) For the following radionuclides only: Mn-54, Mn-56, Co-58, Co-60, Fe-59. For all others, activity is zero. 
TABLE E2

PWR LIQUID WASTES(1)

Waste Source

Containment Bldg. Sump

Aux. bldg. Floor Drains

Laboratory Drains

Sampling Drains

Miscel laneous

Turbine BIdg. Floor Drains

Detergent Wastes

(1aundry, personne1, equipment, and cask cleaning drains)

Condensate Demineralizer

Regenerant Wastes
Fraction of

Flow Rate gpd

40

200

400

35

700

7200

450

3400
Primary Coolant Activity

(1) Based on information presented in PWR-GALE report.

(2) Assuming 292 days/year operation

(3) Table 6.3 is Table 2-20, PWR-GALE report

(4) Main steam activity to demineralizers. All nuclides removed from secondary by demineralizer are removed from resins during regeneration. 
TABLE E3

CALCULATED ANNUAL RELEASE OF RADIOACTIVE MATERIALS

IN UNTREATED DETERGENT WASTE FOR PWR

Nuclide

$\mathrm{Mn}-54$

Co-58

Co-60

Zr-95

$\mathrm{Nb}-95$

Ru-103

Ru-106

$\mathrm{Ag}-110 \mathrm{~m}$

I -131

Cs-134

Cs-137

Ce- 144
$\mathrm{Ci} / \mathrm{yr}$

0.001

0.004

0.009

0.0014

0.002

0.00014

0.0024

0.00044

0.0006

0.013

0.024

0.005

Total

$-0.06$ 
sources and volumes identified in the various SAR's are tabulated here in Table E4. We will use the information presented in this table to identify the likely source of liquid waste in the Surry Plant and to estimate the volume of waste associated with each source. We will provide a basis for selecting the liquid waste source and the estimated quantity of liquid waste associated with each source.

Letdown fluid from the Boron Recovery System is a major source of liquid waste. In the Surry SAR, the quantity is estimated to be $1.23 \times 10^{6} \mathrm{gpy}$, which is 15 percent of the total letdown fluid. The total amount of letdown appears to be based on an estimated annual average letdown flow of $17 \mathrm{gpm}$, which assumes a load-following cycle as indicated in the Surry SAR. For the other plants surveyed, the letdown flow is about one-half of that given for Surry and appears to be based on the assumption the plant is base-loaded rather than load-following. On this basis a more reasonable estimate for the amount of letdown flow liquid waste for the Surry plant would be $6 \times 10^{5}$ gpy. This fluid is assumed to be letdown primary coolant which has been demineralized in the CVCS mixed-bed demineralizer and then held up in the Boron Receovery tanks for processing by the Boron Recovery System evaporators. The distilled effluent from the evaporators is condensed and pumped to the BRS test tanks for sampling. Since this liquid is not required for re-use, it is pumped to the Liquid Waste Disposal System and in particular to the Low Level Waste Drain Tanks. The activity of this liquid waste is assumed to be primary coolant activity reduced by the decontamination factors (DF) associated with the mixed bed demineralizer and the Boron Recovery evaporator. The DF for the demineralizer is assumed to be 10 except for cesium and rubidium, for which the DF is assumed to be 2 as given in the PWR-GALE report (7). 
TABLE E4

ESTIMATED LIQUID WASTE VOLUMES (SAR DATA) (292 DAYS/YEAR OPERATION)

\begin{tabular}{|c|c|c|c|c|c|c|}
\hline Waste Source & $\begin{array}{l}\text { McGuire } \\
\text { (2 units) }\end{array}$ & Ginna & $\begin{array}{l}\text { React } \\
\text { Salem }\end{array}$ & $\begin{array}{l}\text { lant } \\
\text { Oconee } \\
(3 \text { units) }\end{array}$ & $\begin{array}{l}\text { Fort } \\
\text { Calhoun }\end{array}$ & Surry \\
\hline $\begin{array}{l}\text { Reactor Coolant System } \\
\text { Tritium Control }\end{array}$ & 604,400 & -- & 288,000 & $9.03(5)$ & 253,900 & $\begin{array}{c}1.23(6) \\
\text { (BRS Letdown) }\end{array}$ \\
\hline $\begin{array}{l}\text { Sampling \& Laboratory } \\
\text { Drains }\end{array}$ & 35,000 & -- & -- & 7,500 & 59,850 & $\begin{array}{c}92,000 \\
\text { (Sampl ing } \\
\text { Sinks only) }\end{array}$ \\
\hline Demineralizer Sluice & 11,220 & --- & --- & 4,200 & 1,900 & 32,000 \\
\hline Misc. System Leakage & 70,000 & --- & --- & 70,000 & 23,200 & 3,200 \\
\hline Laundry \& Showers & 262,000 & 46,900 & 64,000 & 262,000 & 128,700 & 250,000 \\
\hline Laboratories & --- & 7,800 & 15,600 & --- & --- & 41,000 \\
\hline Equipment Drains, Leaks & --- & 109,200 & 81,000 & -- & --- & --- \\
\hline Decontamination & --- & 25,000 & 16,700 & 3,700 & -- & --- \\
\hline Demineralizer Regeneration & --- & 18,000 & 44,000 & 56,850 & 5,100 & -- \\
\hline Floor Drains & -- & --- & --- & 59,850 & $-\cdots$ & -- \\
\hline
\end{tabular}


The DF for the Boron Recovery evaporator is assumed to be $10^{3}$ for all nuclides except iodine, for which the DF is $10^{2}$, as per the PWR-GALE report $(7)$. In summary, the fraction of primary coolant activity associated with the letdown fluid is given in Table E5. It is also possible to take fluid from the Primary Drain Tank to the High Level Waste Drain Tanks. This indicates that primary coolant, with its attendant higher activity than that identified in Table E5, could end up in High Level Waste Drain Tank.

Sampling liquids from the Auxiliary Building and the Turbine Building are another source of liquid waste. In the Surry SAR, the volume of sampling waste from both sources is estimated to be 92,000 gpy. This estimate is conservative relative to the 36 samples per week at 5 gallons per sample estimate for the three-unit Oconee plant or the 24 samples per day at 5 gallons per sample estimate for the two-unit McGuire plant. The estimated volume of sampling wastes we would use is based on the PWR-GALE Report(7) estimate, 20,500 gpy. This represents Reactor Coolant System Fluid samples and its maximum activity would equal the primary coolant activity. A more reasonable estimate of the sampling activity would account for the fact that each sample volume most likely consists of the liquid sample plus rinse liquid. Based on Sequoyah SAR information, each sample is approximately one-third sample liquid and two-thirds rinse liquid. If the sample liquid is primary coolant, the activity of the liquid waste from sampling would be one-third of the primary coolant activity. The Auxiliary Building Sampling sinks discharge to the Auxiliary Building sump which can be pumped to the High Level, or Low Level Waste Drain Tanks.

Sampling wastes from the Turbine Building would be of lower activity since here the samples would have activities representative of secondary 
steam. We will assume that the volume of Turbine Building sampling waste is equal to that for the Auxiliary Building. Its activity is assumed to be one-third of the secondary steam activity. The Turbine Sampling sinks discharge to floor drains.

Equipment leaks and drains present another source of liquid waste. We will estimate this miscellaneous system leakage at $10 \mathrm{gph}$ or 70,000 gpy. This value was used as the basis for estimates made in the McGuire and Oconee SAR's. This is also consistent with the $200 \mathrm{gpd}$ estimate of liquid waste from the Auxiliary Building floor drains given in the PWR-GALE report. As indicated in that report, the activity of this waste is assumed to be 10 percent of the primary coolant activity. As indicated previously, the Auxiliary Building sump can be pumped to the High Level or Low Level Waste Drain Tanks depending on the waste activity level.

Laboratory drains are also a source of liquid waste. The volume of waste associated with lab drains is estimated to be 41,000 gpy as given in the Surry SAR. This waste is pumped to the High Level Waste Drain Tanks. Its activity is estimated to be 0.3 percent of the primary coolant activity.

Reactor coolant leak-off from pump seals and valve stems can represent a source of liquid waste. We estimate the leak-off at 40 gpd per reactor or 23,400 gpy. This estimate corresponds to leak-off estimates provided in the Fort Calhoun SAR. This waste fluid drains to the Primary Drain Tanks and is processed in the Boron Recovery System prior to disposal. The activity level associated with this fluid is estimated in Table E5.

Drainage to the containment sump is a source of liquid waste. The PWRGALE report estimates the volume associated with this source to be $40 \mathrm{gpd}$ per reactor or for the Surry Plant, 23,400 gpy. We will assume the activity of this waste is equivalent to primary coolant activity. 
Detergent wastes and decontamination wastes represent sources of 1 iquid drained to the LWDS. In the PWR-GALE report, (7) these are estimated to total 450 gpd per reactor with the yearly activity released given in Table E3. We have chosen to separate this estimated flow as applied to surry since in Surry the laundry wastes are pumped to the contaminated drains tanks while the decontamination tanks drain to the low level waste drain tanks. The total detergent and decontamination waste yearly total of 250,000 gallons consists of 230,000 yallons of detergent waste and 20,000 gallons of decontamination wastes. The bases for the decontamination waste estimate are waste volume estimates given in the Salem and Ginna SAR's.

As indicated in the PWR-GALE report, (7) turbine building floor drains are also a major source of liquid wastes. The estimated leakage rate of 7,200 upd per reactor is derived from experience at Yankee Rowe. For the Surry Plant, the total liquid waste from turbine building drains would be $4.2 \times$ $10^{6} \mathrm{gpy}$. This waste source would have an activity level equivalent to that of secondary steam. This waste would be directed to the Vent and Drain System and then to the high level or low level waste drain tanks depending on its activity level. Table E6 summarizes these estimated waste volumes and their associated activity levels.

Based on the Surry Vent and Drain System classification scheme, we would anticipate that all wastes having an activity arising from primary coolant would be directed to the high level waste drain tanks. The wastes involving secondary steam activity and decontamination wastes would be directed to the low level waste drain tanks, and the laundry wastes would be directed to the contaminated drains tanks.

Based on Table E6 estimates, the total daily effluent to the two high level waste drain tanks is 2,665 gallons, which is slightly larger than the 


\title{
TABLE E5
}

\author{
Estimated Fraction of Primary Coolant Activity \\ For Various Nuclides in Boron Letdown Stream \\ Nuclide \\ Iodine \\ Cesiun, Rubidiun \\ All Others \\ Fraction $P C A$ \\ 0.001 \\ 0.0005 \\ 0.0001
}

TABLE E6

ESTIMATED ANNUAL LIQUID WASTE VOLUMES

AND ASSOCIATED ACTIVITY LEVELS

Source

Boron Recovery System
Letdown Fluid.

Sampling Drains, Auxiliary Building

Sampling Drains, Turbine Building

Equipment Leaks and

Drains, Auxiliary

Building Drains

Laboratory Drains

Reactor Coolant Leakoffs

Containment Building Sump

Detergent Wastes

Decontamination Wastes

Turbine Building Drains
Volume (gal) Activity Leve]

$600,000 \quad$ See Table 5

$20,500 \quad 0.33$ of Prinary Coolant Activity

20,500

0.33 of Secondary Steam Activity

70,000

0.10 of Primary Coolant Activity

41,000

0.003 of Primary Coolant Activity

23,400

See Table 5

23,400

Primary Coolant Activity

230,000

Table 2-19 PWR-GALE Report

20,000

Table 2-19 PWR-GALE Report

Secondary Steam Activity 
single tank capacity of 2,390 gallons. This waste is then pumped to the waste disposal evaporator, where it is evaporated and the distillate pumped eventually to the waste disposal evaporator test tanks. The decontamination factor for the evaporator is assumed to be $10^{4}$ for all nuclides except iodine, for which it is $10^{3}$. The feed to concentrate volume reduction associated with the evaporator is 40 to 1 .

The volume of waste in the various tanks at any time and its associated activity would depend on the specific delivery cycle for each waste effluent. We have no information on this. We will assume a uniform delivery of wastes and thus will estimate an average activity level for the liquid waste entering the waste disposal evaporator from the high level drain tanks. This average activity is determined as follows:

$$
\bar{A}=\Sigma Q_{i} A_{i} / \Sigma Q_{i}
$$

where $\bar{A}=$ the average activity for the waste entering the waste disposal evaporator

$Q_{j}=$ the waste flows entering the high level waste drain tanks

$A_{i}=$ activity associated with $Q_{j}$.

For the high level waste drain tanks, the average activity is estimated to be 5 percent of the primary coolant. Slightly larger activity levels are predicted for iodine nuclides, and have been accounted for in this estimate. Applying the waste disposal evaporator decontamination factor to the estimated influent given above, we estimate the volume of waste pumped to the waste disposal evaporator test tanks is $2600 \mathrm{gpd}$, accounting for the evaporator volume reduction with an activity level of $5 \times 10^{-6}$ times the primary coolant activity for all nuclides except iodine which has an activity level estimated to be a factor of ten larger. 
The waste volumes and activity levels for the low level waste drain tanks can be estimated in a similar fashion. The total daily flow to these tanks, based on the estimates provided in Table E6 is estimated to be 14,500 gallons. The activity associated with this flow is essentially the secondary steam activity, since the turbine building drain flow dominates our flow estimate.

The estimated waste influent to the contaminated drain tanks can be obtained directly from Table E6. since only laundry wastes are directed to these tanks. The associated activities are given in Table E3.

Primary coolant and secondary steam activities have been estimated using PWR-GALE report information. Activities for the various nuclides are tabulated in Table E7. For the nuclides of interest (as per Section 11a of Chapter 11 of the Surry SAR) the total primary coolant activity at equilibrium is $1.11 \mu \mathrm{Ci} / \mathrm{g}$. The total secondary stean activity at equilibrium is $4.9 \mathrm{x}$ $10^{-7} \times \mathrm{Ci} / \mathrm{g}$. Since the operating conditions for these tanks are atmospheric pressure and $120 \mathrm{~F}$, on a volumetric basis, these activities are $1.11 \mu \mathrm{Ci} / \mathrm{cc}$ and $4.9 \times 10^{-7} \mu \mathrm{Ci} / \mathrm{cc}$, respectively.

As Table E7 shows, the bulk of the activity is contributed by the iodine nuclides (approximately 85 percent of the primary coolant activity and 90 percent of the secondary steam activity). Since these nuclides have relatively short half-lives, radioactive decay during liquid waste holdup can have some effect on the amount of activity released. Although we have not estimated a likely hold-up time for decay in the system, we have considered the effect of a 12 hour hold-up and found that the waste activity could be reduced by a factor of about two.

We will now estimate the activities in the high level waste drain tanks, the waste evaporator test tanks, and the low level waste drain tanks based on 
the estimated waste volumes and associated activities presented here. The high level waste drain tanks receive an estimated daily influent of 2,665 gal1ons. The activity of this waste is estimated to be 5 percent of the primary coolant activity, or $0.056 \mathrm{\mu Ci} / \mathrm{cc}$. The total activity in the tank, based on a tank volume of 2390 gallons, is $0.5 \mathrm{Ci}$. In the evaporator waste test tank, the activity level is reduced by the waste disposal evaporator decontamination factor of $10^{4}$ for all nuclides except iodine, for which the activity is reduced by the factor $10^{3}$. In the test tank, the activity level of the iodine nuclides is $9.5 \times 10^{-4} \mu \mathrm{Ci} / \mathrm{cc}$ and for the other nuclides the activity level is $1.6 \times 10^{-5} \mu \mathrm{Ci} / \mathrm{Cc}$ for a total activity of $9.7 \times 10^{-4} \mu \mathrm{Ci} / \mathrm{cc}$. If we assume the test tank is full (capacity 548 gal), the tank activity is 0.002 Ci. The two low level waste drain tanks each have a volume of 2,874 gallons. If a tank is full of liquid waste of the activity level estimated here for secondary steam, the tank activity is $5.4 \times 10^{-6} \mathrm{Ci}$. The two contaminated drain tanks have a volume of $1,230^{\circ}$ each. If a tank is filled with laundry and decontamination waste with the activity level based on Table E3 activities, the tank would hold about $3 \times 10^{-4} \mathrm{Ci}$.

The estimated tank activities presented here are based on our interpretation of the operation of the Surry Plant Liquid Waste Disposal System, using Surry SAR information as the basis. In carrying out these estimates, it was quite apparent that large variations in the various tank activity levels were possible depending on the assumptions made concerning the disposition of the liquid wastes. For example, the system piping can accommodate the transfer of primary coolant from the Primary Drain Tank to the High Level Waste Drain Tanks, although this does not appear to be a normal or typical operation. 
TABLE E7

PRIMARY COOLANT AND SECONDARY STEAM ACTIVITIES OBTAINED USING PWR-GALE REPORT ADJUSTMENT FACTORS

\begin{tabular}{|c|c|c|c|c|c|c|c|c|}
\hline I sotope & $\begin{array}{c}\text { PWR-GALE } \\
\text { Pri. Coolant } \\
\text { Activity } \\
\mathrm{C} \mathrm{C} / \mathrm{g}\end{array}$ & $\begin{array}{c}\text { Half-life } \\
\text { days }\end{array}$ & $\begin{array}{c}\text { Decay } \\
\text { Constant } \\
\lambda, \mathrm{hr}\end{array}$ & $\begin{array}{l}f^{\prime} \\
- \\
\end{array}$ & $\begin{array}{c}\text { Mod. PWR-GALE } \\
\text { Pri. Cool ant } \\
\text { Activity } \\
\text { Ci/g }\end{array}$ & $f_{\text {sec }}$ & $\begin{array}{c}\text { PWR-GALE } \\
\text { Sec. Steaam } \\
\text { Activity } \\
\mu \mathrm{Ci} / \mathrm{g} \\
\end{array}$ & $\begin{array}{c}\text { Mod. PWR-GALE } \\
\text { Sec. Steam } \\
\text { Activity } \\
\mu \mathrm{Ci} / \mathrm{g} \\
\end{array}$ \\
\hline$M n-54$ & $3.1(-4)$ & $3.121(2)$ & $9.25(-5)$ & .905 & $2.8(-4)$ & 4.66 & $2(-11)$ & $9.3(-11)$ \\
\hline $\mathrm{Co-58}$ & $1.6(-2)$ & $7.161(1)$ & $4.03(-4)$ & .905 & $1.4(-2)$ & 4.66 & $8(-10)$ & $3.7(-9)$ \\
\hline $\mathrm{Fe}-59$ & $1.0(-3)$ & $4.506(1)$ & $6.41(-4)$ & .905 & $9.1(-4)$ & 4.66 & $6(-11)$ & $2.8(-10)$ \\
\hline Co-60 & $2.0(-3)$ & $1.919(3)$ & $1.51(-5)$ & .905 & $1.80(-3)$ & 4.66 & $9(-11)$ & $4.2(-10)$ \\
\hline$S r-89$ & $3.5(-4)$ & $5.109(1)$ & $5.65(-4)$ & .905 & $3.2(-4)$ & 4.66 & $2(-12)$ & $9.3(-12)$ \\
\hline$S n-90$ & $1.0(-5)$ & $1.044(4)$ & $2.77(-5)$ & .905 & $9.1(-6)$ & 4.66 & $4(-13)$ & $1.9(-12)$ \\
\hline$S n-91$ & $6.5(-4)$ & $4.051(-1)$ & $7.13(-2)$ & .98 & $6.4(-4)$ & 3.26 & $2(-11)$ & $6.5(-11)$ \\
\hline$Y-90$ & $1.2(-6)$ & 2.665 & $1.08(-2)$ & .92 & $1.1(-6)$ & 4.28 & $8(-14)$ & $3.4(-13)$ \\
\hline$Y-91$ & $6.4(-5)$ & 5.898 & $4.9(-3)$ & .91 & $5.9(-5)$ & 4.46 & $3(-12)$ & $1.2(-11)$ \\
\hline$Z r-95$ & $6.0(-5)$ & $6.521(1)$ & $4.43(-4)$ & .905 & $5.4(-5)$ & 4.66 & $4(-12)$ & $1.9(-11)$ \\
\hline $\mathrm{Nb}-95$ & $5.0(-5)$ & $3.503(1)$ & $8.24(-4)$ & .905 & $4.5(-5)$ & 4.66 & $4(-12)$ & $1.9(-11)$ \\
\hline Mo-99 & $8.4(-2)$ & 2.795 & $1.03(-2)$ & .92 & $7.8(-2)$ & 4.3 & $4(-9)$ & $1.7(-8)$ \\
\hline$I-131$ & $2.7(-1)$ & 8.061 & $3.58(-3)$ & .912 & $2.5(-1)$ & 2.04 & $6.8(-8)$ & $1.4(-7)$ \\
\hline
\end{tabular}


TABLE E7 (Cont'd)

PRIMARY COOLANT AND SECONDARY STEAM ACTIVITIES OBTAINED USING PWR-GALE REPORT ADJUSTMENT FACTORS

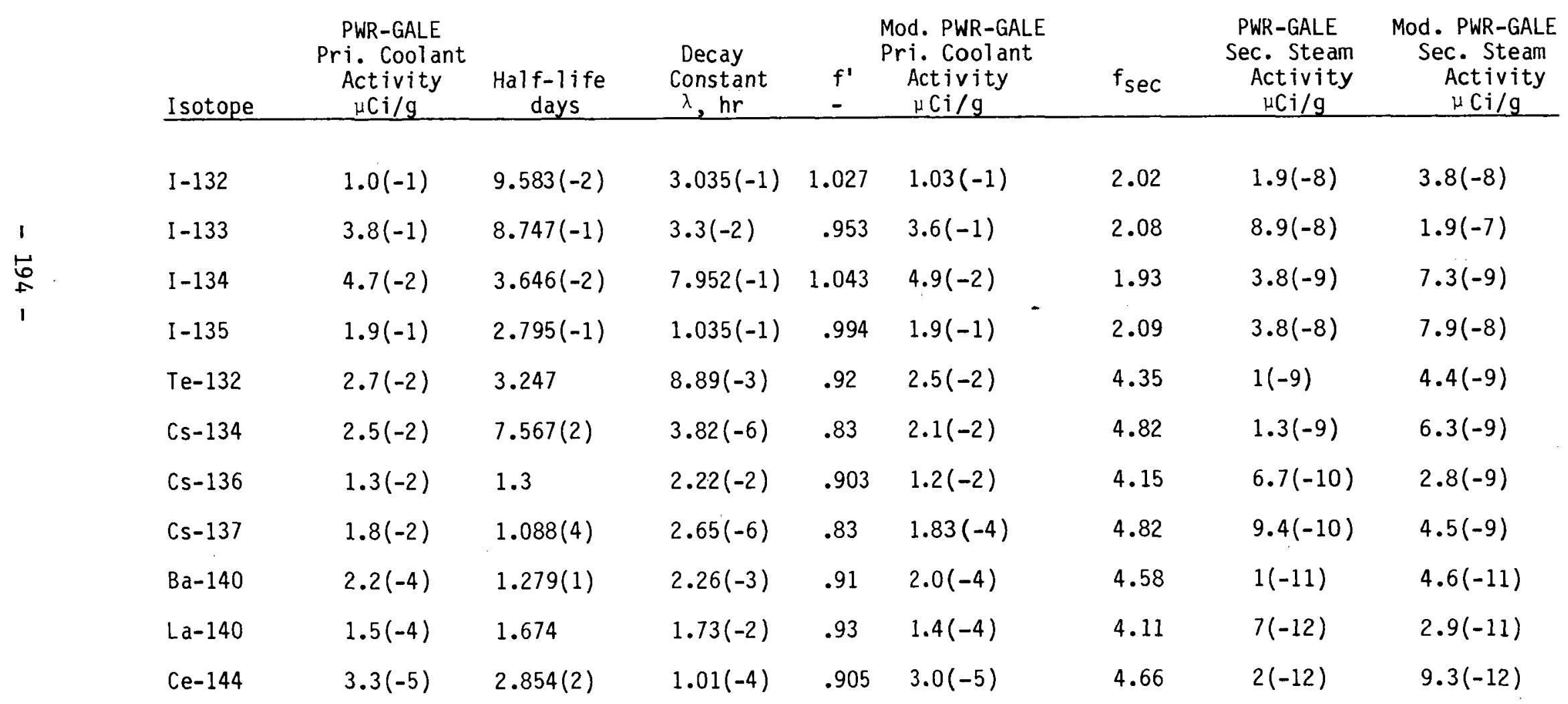


It is our feeling that information should be obtained from the Surry Plant on the typical activity levels in the various Liquid Waste Disposal Systern tanks and also on the actual mode of operation of the Waste Disposal System. 
APPENDIX F

MODELS FOR IODINE SPIKING AND
I-131 RELEASES DURING A LOSS
OF OFFSITE POWER SHUTDOWN

\section{F.1 Introduction}

This Appendix yives a model for the calculation of $1-131$ releases to the atmosphere during a shutdown following by loss of offsite power (LOSP). The release depends (amongst other parameters) on:

1. The duration $\tau$ of the LOSP event.

2. The "clad defect level," $f_{c d}$, defined as the fraction of core power generated by fuel rods with clad defects.

3. The magnitude $Q_{\ell}$ (lbs/hr) of the primary-to-secondary leak in the steam generators.

4. The time interval, $t_{1}$, between the onset of the LOSP event and the time of the immediately preceding reactor startup.

Each of the variables mentioned $\left(\tau, f_{c d}, Q_{\ell}, t_{1}\right)$ is a random variable. Appropriate distributions for these parameters will be defined.

The I-131 release is affected by the so-called "iodine spiking" $(14,15,34,35)$ phenamenon. This phenomenon refers to the observed increase in the release rate of iodine from fuel to primary coolant, which occurs during power reductions, and is discussed in the following section. Iodine spiking can affect the I-131 atmospheric release in a LOSP event in two ways. First of al1, iodine spiking caused by the LOSP shutdown itself will affect the I-131 release, especially if the shutdown is sufficiently prolonged so that the radioiodine concentration in the primary coolant increases appreciably; this 
in turn increases the radioiodine concentration on the secondary side of the steam generators. Secondly, depending on the time interval $t_{1}$ between the LOSP event and the startup immediately preceding the LOSP event, the I-131 activities in the primary and secondary coolants may, at the onset of the LOSP shutdown, be much higher than their steady-state values; this is a consequence of the iodine spiking caused by the shutdown preceding the LOSP incident. Thus, for certain ranges in the value of $t_{1}$, the release of $I-131$ to the atmosphere will be appreciably larger than would be the case if $t_{1}$ were so large that the steady-state values of I-131 activity were attained in the primary and secondary coolant.

The radioiodine release which occurs during a LOSP event occurs through the steam generator safety and relief valves. It will be terminated when the LOSP event is terminated, since the main condenser may be used for heat rejection when offsite power is restored. Moreover, after a time period on the order of 6 hours, heat removal from the primary system can be accomplished by the Residual Heat Removal System, without venting steam to the atmosphere. Thus, the radioiodine release to the atmosphere will be terminated after about 6 hours, even if the LOSP event extends beyond this period of time.

\section{F.2 Iodine Spiking}

A well known observation is that the activity of certain nuclides in the reactor coolant increases when there is a power reduction in a reactor which has operated with cladding defects for some time. $(14,15,34,35)$ The increase in activity has also been observed during power increase and primary system depressurizations. In particular, radioiodine isotopes exhibit this behavior and this phenomenon has come to be known as "iodine spiking." In this report 
jodine spiking has been considered in the analys is of a number of accidents due to its potential for increasing the radioiodine release to the environment. Operating data(15) show that the release rate of iodine from fuel to coolant can temporarily increase by as much as three orders of magnitude (over the steady-state release rate) on reactor shutdown. The experimental data on iodine (I-131 only) spiking has been utilized in two ways in this report, designated as Models $A$ and $B$. Since in some applications the variation of the primary coolant activity with time is required, Model $A$, whereas in other applications only the total arnount of Iodine released in the spike is required, Model B. The ensuing discussion shows the relationship between these two models, the data and also the models used by other authors.

Iodine Spiking Models:

Model A

Following each reactor shutdown, the release rate of iodine from fuel to the primary coolant increases by a factor of a over the steady-state release rate for a period $\Delta t$, and then drops down to the steady-state release rate. During the course of the spike, i.e., a time period of $\Delta t$, the primary coolant activity can be expressed by

$$
\frac{d}{d t} A_{p}(t)=\alpha R_{0}-\lambda_{p} A_{p}(t) \quad 0 \leq t \leq \Delta t
$$

The initial condition is that at $t=0, A_{p}(0)=R_{0} / \lambda p$. It is assumed that the iodine removal constant $\lambda_{p}$ remains the same during the spiked and unspiked conditions, and

$$
A_{p}(t)=\text { primary coolant activity in } \mu \mathrm{C} ; \text {, }
$$

$\alpha=$ spiking factor assumed constant within the spike duration interval 
$R_{0}=$ equilibrium release rate of iodine from fuel to primary coolant during steady-state full power operation in $\mu \mathrm{Ci} / \mathrm{sec}$,

$\Delta t=$ spike duration time,

$\lambda_{p}=$ total removal rate of iodine,

$=\lambda_{d}+\lambda_{p u}$

$\lambda_{d}=$ radioactive decay constant

$\lambda_{p u}=F\left(1-\frac{1}{D F}\right) / M_{p}=$ purification constant

$F=$ purification mass flow rate,

DF = decontamination factor associated with the purification process, and

$M_{p}=$ mass of the primary coolant.

The solution of Equation F.1 is

$$
A_{p}(t)=\frac{R_{0}}{\lambda_{p}}\left[\alpha-(\alpha-1) e^{-\lambda_{p} t}\right] \quad 0 \leq t \leq \Delta t
$$

The primary coolant activity concentration during spiking is

$$
a_{p}(t)=a_{p o}\left[\alpha-(\alpha-1) e^{-\lambda} t\right] \quad 0 \leq t \leq \Delta t
$$

where

$$
\begin{aligned}
a_{p_{0}} & =R_{0} /\left(\lambda_{p} M_{p}\right)=\text { equilibrium primary coolant activity concentration } \\
& \text { in } \mu \mathrm{Ci} / g m
\end{aligned}
$$

The total amount of iodine released during the spike, $Q(C i)$ is

$$
Q=10^{6} \alpha R_{0} \Delta t
$$

The numerical values for the model parameters chosen are:

$\alpha=500$ (from NRC Standard Revi ew Plan, Sec. 15.1.5),

$\Delta t=2$ hours (from Fontecilla and Grimes(17), and 


$$
\lambda_{p}=\frac{F}{M_{p}}\left(1-\frac{1}{D F}\right)+\lambda_{d} \approx 0.07 \mathrm{hr}^{-1}
$$$$
\mathrm{F}=4670 \mathrm{~g} / \mathrm{sec},
$$$$
M=2.5 \times 10^{8} \mathrm{~g} \text {, }
$$

DF is assumed to be infinity, and

$$
\lambda_{d}=9.96 \times 10^{-7} \mathrm{sec}^{-1} \text {. }
$$

These values imply that $R_{0}=4900 a_{p o}$.

According to Neeb and Schuster, $(30)$ the iodine spike in a PWR results in a maximum value of the primary I-131 activity equal to between 50 and 100 times the equilibrium value; moreover, this maximum value occurs between 60 to 90 minutes after the beginning of the spike. Thus, according to Reference (30), $\Delta t$ is between 1 hour and 1.5 hours. The NRC spiking model ( $i . e ., \alpha=500, t=2$ hours) yields a maximum value of 66 , as shown below, for the primary coolant activity concentration at the end of spike duration $\Delta t$ in comparison with the equilibrium primary concentration.

$$
\frac{a_{p}(t)}{a_{p o}}=\left(500-499 e^{-(0.07)(2)}\right) \approx 66
$$

Therefore, the NRC model in comparison to that of Neeb and Schuster, (30) which gives the above ratio to be 50 to 100 , appears not to be conservative, but rather, in the neighborhood of the best estimate values.

\section{Model B}

An empirical correlation between the total activity release to the primary coolant during a spiking sequence and the equilibrium release rate obtained by a straight line fit to the data tabulated by Pasedag(15) is given by

$$
\begin{aligned}
Q= & e^{0.70 \pm 1.05} R_{0} \\
& -200-
\end{aligned}
$$


where

$$
\begin{aligned}
Q= & \text { total release of } I-131 \text { in a spiking sequence }(\mathrm{C} i), \\
R_{0}= & \text { equilibrium release rate observed prior to spiking sequence }(\mu \mathrm{Ci} / \mathrm{sec}) \text {. } \\
& \text { This has been defined earlier in relation to Equation } F .1 .
\end{aligned}
$$

$\mathrm{e}^{0.70 \pm 1.05}=$ proportionality constant $(\mathrm{ci}-\mathrm{sec} / \mathrm{Ci})$. The variation in the exponent represents the distribution of observed spikes.

Comparison of Model A and Model B

The two models may be compared by using Equations F.4 and F.6 for the quantity of I released during the spike.

Eliminating $R_{0}$ gives

$$
\left(3.6 \times 10^{-3}\right) \text { a } \Delta \text { t hrs }=e^{0.70 \pm 1.05}
$$

By substituting $\Delta t=2$ hours, the value chosen in Model $A$, the range of $\alpha$ which reflects the variation in the exponent in the R.H.S. of Equation F.7 is For $\Delta t=2$ hours:

$$
\begin{aligned}
& \alpha=98 \text { for } \mathrm{e}^{0.70-1.05} \rightarrow \text { Lower bound } \\
& \alpha=280 \text { for } \mathrm{e}^{0.70} \rightarrow \text { Central value } \\
& \alpha=799 \text { for } \mathrm{e}^{0.70+1.05} \rightarrow \text { Upper bound }
\end{aligned}
$$

For $\alpha=500$ and $\Delta t=2$ hours, values chosen in Model A, Equation F. 4 can be alternatively written in the same form as Equation F.6 of Model B to give

$$
Q=e^{1.28} R_{0}
$$

From the above discussion, it is apparent that choosing $\alpha=500$ and $\Delta t=2$ hours provides a correlation between $Q$ and $R_{0}$ which is somewhere between the central value and the upper bound obtained in Model B. In parenthesis, it 
might be noted that a bounding correlation given by Pasedag(15) is

$$
Q=10 R_{0}=e^{2.30} R_{0}
$$

Figure F.1 shows spiking data from Pasedag(15) and compares Model $A$ and Model B. The figure shows to what extent NRC's spiking model is valid in light of data tabulated in Reference 41. Pasedag's bounding line given by Equation $F .10$ is also shown as a comparison.

\section{F.3 Calculational Model for I-131 Release}

The time* at which the LOSP reactor trip occurs is denoted by $t_{1}$. The time $t=0$ denotes the time of the reactor startup immediately preceding the LOSP trips. At the time $t=0$ the reactor has reached full power. During the shutdown immediately preceding this startup, there has been, according to our iodine spiking model $A$, an increase (by a factor $\alpha$ ) in the release rate of I-131 from the fuel to the primary coolant for a period $\Delta t$, with the release rate then dropping back to its steady-state value. At the end of this time period $\Delta t$ the primary coolant $[-131$ activity will have attained its maximum value, and it will then decrease. The rate of this decrease will depend on whether or not offsite power is available during the shutdown. If offsite power is not available, there is no removal of iodine by purification flow of primary coolant through demineralizers, and the removal of I-131 activity will decrease at the relatively slow rate dictated by the 8.05 day startup will be assumed to be caused by some event other than loss of offsite power; out of some 10 reactor trips per year only about .2/yr are caused by LOSP (Reactor Safety Study data), (1) so this is a good assumption. (The results are not *Times of interest are defined in Table F1, as well as in the text, for the reader's convenience. 


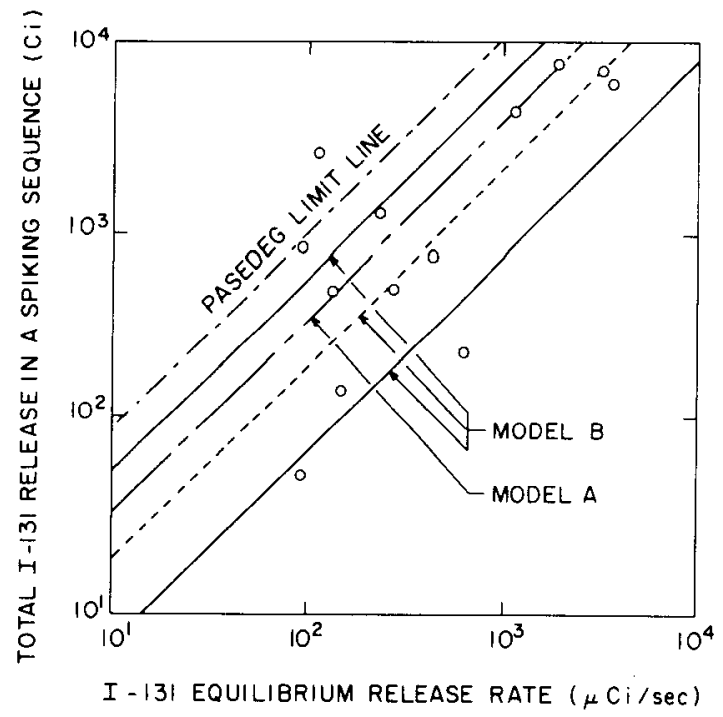

Figure Fl. Experimental data on iodine spiking compared with severa1 theoretical models

\title{
TABLE F1
}

\section{TIMES OF INTEREST}

\author{
$t=t_{0}$ : Onset of reactor shutdown immediately preceding LUSP \\ shutdown; iodine spike begins here. \\ $t=0: \quad$ Reactor is brought up to power after shutdown initiated at \\ $t=t_{0}$. \\ $t=t_{1}: \quad$ LOSP trip occurs; atmospheric release begins; iodine \\ spike due to LOSP trip begins. \\ $t=t_{1}+t: \quad$ Spike ends. \\ $t=t_{1}+:$ Atmospheric release terminates, either by restoration of \\ offsite power or by use of Residual Heat Removal System.
}


sensitive to this assumption anyway). The I-131 activity in the primary coolant at $t=0$ will be assumed to be that at the end of the spike, without taking into account the facts that if the shutdown were fairly extended some of this activity would have a change to be removed by the purification flow. Results are not sensitive to this assumption.

In order to calculate the iodine activities in the primary and secondary coolant, we shall use Model A which has been described in the last section. The value of $a_{p}$ at the time of startup, $t=0$, is the value obtained from Equation $F .3$ for $t-t_{0}=\Delta t$, since we are considering a spike of duration $\Delta$ t. Thus

where

$$
a_{p}(0)=(1+\beta) a_{p o}
$$

$$
1+\beta=\alpha-(\alpha-1) e^{-\lambda} p^{\Delta t}
$$

The value of the specific activity of I-131 in the secondary coolant, $a_{s}(0)$, is required. At the time $t_{0}$ of the shutdown immediately preceding the LOSP shutdown, the secondary coolant activity is assumed to be at its equilibrium level; any transients prior to the time $t=t_{0}$ are neglected. During the time interval $t_{0} \leq t \leq 0$ the secondary coolant activity satisfies

$$
\frac{d A_{s}}{d t}=Q_{\ell} a_{p}-\lambda_{s} A_{s}
$$

where $\lambda_{S}$ is the rate constant for removal of I-131 from the secondary coolant, and $Q_{\ell}$ is the primary-to-secondary leak rate (in lbs/hr). The rate constant $\lambda_{S}$ is given by

$$
\lambda_{s}=\frac{F_{b}}{M_{s}}+\lambda_{d}
$$


where $F_{b}$ is the blowdown flow rate (to the blowdown flash tank). By dividing Equation $F .13$ through by $M_{S}$, the mass of the water on the secondary side of the steam generators, one obtains

$$
\frac{d a_{s}}{d t}=\frac{Q_{l}}{M_{s}} a_{p}-\lambda_{s} a_{s}
$$

where

$$
a_{s}=\frac{A_{s}}{M_{s}}
$$

is the specific activity of I-131 in the water on the secondary side of the steam generator. During the time interval $t_{0} \leq t \leq 0$ the quantity ap is given by Equation F.3. Inserting the expression for $a_{p}$ from Equation F.3 into Equation F.14 one obtains

$$
\frac{d a_{s}}{d t}=a_{p o} \frac{Q_{l}}{M_{s}}\left[\alpha-(\alpha-1) e^{-\lambda_{p}\left(t-t_{0}\right)}\right]-\lambda_{s} a_{s}
$$

The solution of this equation is

$$
\begin{aligned}
a_{s} & =a_{s 0} e^{-\lambda_{s}\left(t-t_{0}\right)}+a_{s 0} \alpha\left(1-e^{-\lambda_{s}\left(t-t_{0}\right)}\right) \\
& +a_{s 0} \frac{(\alpha-1) \lambda s}{\lambda_{p}-\lambda_{s}}\left(e^{-\lambda_{p}\left(t-t_{0}\right)}-e^{-\lambda_{s}\left(t-t_{0}\right)}\right)
\end{aligned}
$$

where the initial condition that $a_{s}=a_{s o}$ at $t=t_{0}$ used. This means that at the start of the spike the secondary coolant activity is at its equilibrium level. (The time $t_{0}$, it is to be recalled, refers to the onset of the spike caused by the shutdown before the LOSP shutdown). In addition, in order to obtain Equation F.16, apo was eliminated by means of the relationship 


$$
a_{s o}=\frac{Q_{l}}{M_{s}} \quad \frac{a_{p 0}}{\lambda_{s}}
$$

This relationship is easily derived by a steady-state activity balance on the secondary side of the steam generator. The amount of activity flowing into the secondary under steady-state conditions is $Q_{\ell} a_{p o}$, while the amount being removed is $\lambda M_{S} a_{S O}$. Equating these two quantities one obtains Equation F.17. Differences in the vailue of $M_{S}$ from full load to no load conditions are neglected.

It is now necessary to calculate the values of $a_{p}(t)$ and $a_{s}(t)$ in the time intervals $\left(0, t_{1}\right)$ between the time the reactor has been brought up to power at $t=0$ to the time of the LOSP trip at time $t=t_{1}$. During this period the release rate of I-131 from the fuel to the primary coolant is the unspiked steady state value of $R_{0}$. The primary coolant activity $A_{p}$ during the time interval $\left(0, t_{1}\right)$ therefore satisfies

$$
\frac{d A_{p}}{d t}=R_{0}-\lambda_{p} A_{p}
$$

Solving Equation $F .18$, one obtains for the specific activity $a_{p}=$ $A_{p} / M_{p}$ the value

$$
a_{p}=\frac{R_{0}}{\lambda_{p} M_{p}}+\left(a_{p}(0)-\frac{R_{0}}{\lambda_{p} M_{p}} e^{-\lambda_{p} t}\right.
$$

or using Equation F.11

$$
a_{p}=a_{p o}+\beta a_{p o} e^{-\lambda_{p} t}
$$


We now calculate $a_{s}(t)$ during the time interval $\left(0, t_{1}\right)$. During this time interval $a_{s}(t)$ satisfies the differential Equation H.14 with $a_{p}$ given by Equation F.20. Thus

$$
\frac{d a_{s}}{d t}=\frac{Q_{\ell} a_{p o}}{M_{s}}\left(1+\beta e^{-\lambda p t}\right)-\lambda_{s} a_{s}
$$

The solution of Equation F.21 is

$$
a_{s}=\frac{Q_{\ell}}{\lambda_{s} M_{s}} a_{p o}+\frac{Q_{\ell} \beta a_{p o}}{M_{s}\left(\lambda_{s}-\lambda_{p}\right)} e^{-\lambda_{p} t}+c e^{-\lambda_{s} t}
$$

where $c$ is a constant to be determined by the initial condition at $t=0$. Making use of Equation F.17 to relate $a_{\text {po }}$ to $a_{S O}$, and using the initial condition one can write Equation F.22 in the form

$$
a_{s}(t)=a_{s 0}+\beta a_{s o} \frac{\lambda_{s}}{\lambda_{s}-\lambda_{p}}\left(e^{-\lambda_{p} t}-e^{-\lambda_{s} t}\right)+\left(a_{s}(0)-a_{s o}\right) e^{-\lambda_{s} t}
$$

Here $a_{s}(0)$ is obtained from Equation F.17; since the spike duration is $\Delta t$. By evaluating Equation F.23 at $t=t_{1}$ one obtains $a_{s}\left(t_{1}\right)$, the secondary coolant specific I-131 activity at the start of the LOSP incident.

We now calculate the quantities $a_{p}(t)$ and $a_{s}(t)$ during the time inter$\operatorname{val}\left(t_{1}, t_{1},+\tau\right)$ : this time interval corresponds to the time during which the atmospheric radioactivity release occurs, since $t_{1}$ is the time at which the LOSP trip occurs, and $\tau$ is the duration of the LOSP event. (The time $t_{1}+\tau$ corresponds to the time at which the atmospheric release is terminated, residual heat removal system). At the time $t_{1}$ a spike in the release rate of $I-131$ from the fuel to the primary coolant occurs; this spike, like the spike that occurred in the time interval $\left(t_{0}, 0\right)$, is assumed to last for a time $\Delta t$, and to correspond to an increase in the release rate by a 
factor of $\alpha$ over the steady state release rate. This transient, unlike the turbine trip type of transient assumed at time $t_{0}$, is a LOSP transient;

since offsite power is not available there is no purification flow through the demineralizers. Thus the rate constant for removal of I-131 is simply that corresponding to the radioactive decay half life of 8.05 days. This time constant is long compared to the duration $\Delta t$ of the spike and to the duration $\tau$ of the offsite power event. Thus it is possible to neglect the removal processes for I-131 from the primary coolant during the time of the LOSP event. Then $a_{p}$ satisfies

$$
\frac{d a_{p}}{d t}=\frac{\alpha R_{0}}{M_{p}}
$$

during the time interval $\left(t_{1}, t_{1}+\Delta t\right)$. The solution of this equation is

$$
a_{p}=\frac{\alpha R_{0}}{M_{p}}\left(t-t_{1}\right)+a_{p}\left(t_{1}\right)
$$

or

$$
a_{p}=\alpha a_{p o} \lambda_{p}\left(t-t_{1}\right)+a_{p}\left(t_{1}\right)
$$

In Equation $F .25, a_{p}\left(t_{1}\right)$ is given by Equation $F .20$, evaluated for $t=t_{1}$. During this same time interval $\left(t_{1}, t_{1}+\Delta t\right)$ it is also a good approximation to neglect removal processes for I-131 from the secondary system. Then

$$
\frac{d a_{s}}{d t}=\frac{Q_{l}}{M_{s}} a_{p}
$$

or using Equation F.25,

$$
\frac{d a_{S}}{d t}=\frac{Q_{l}}{M_{S}} \quad \alpha a_{p o} \lambda_{p}\left(t-t_{1}\right)+a_{p}\left(t_{1}\right)
$$


The solution of Equation F.28 is

$$
a_{s}(t)=1 / 2 \frac{Q_{\ell}}{M_{S}} \quad \alpha a_{p o} \lambda_{p}\left(t-t_{1}\right)^{2}+\frac{Q_{\ell}}{M_{s}} a_{p}\left(t_{1}\right)\left(t-t_{1}\right)+a_{s}\left(t_{1}\right)
$$

Here $a_{s}\left(t_{1}\right)$ is given by Equation $F .23$, evaluated for $t=t_{1}$.

Equation $F .29$ gives $a_{S}(t)$ from the time $t_{1}$ of the onset of the LOSP event to the time $t_{1}+\Delta t$. For LOSP events so prolonged in time that $\tau>\Delta t$ it is necessary to calculate the values of $a_{p}(t)$ and $a_{s}(t)$ in the time interval $\left(t_{1}+\Delta t, t_{1}+\tau\right)$. During this time interval the atmospheric release is still continuing but the release rate of iodine from fuel to primary coolant has dropped back to its unspiked value (i.e., it is back to the steady state release rate. During the time interval $\left(t_{1}+\Delta t, t_{1}+\tau\right)$ the quantities $a_{p}$ and $a_{s}$ satisfy (again neglecting $I-131$ removal processes from the primary and secondary coolants):

$$
\begin{aligned}
& \frac{d a_{p}}{d t}=\frac{R_{0}}{M_{p}}=\lambda_{p} a_{p o} \\
& \frac{d a_{s}}{d t}=\frac{Q_{l}}{M_{s}} a_{p}
\end{aligned}
$$

The solution of Equation F.30 is

$$
a_{p}=\lambda_{p} a_{p o}\left(t-t_{2}\right)+a_{p}\left(t_{2}\right)
$$

where $t_{2}=t_{1}+\Delta t$, and $a_{p}\left(t_{2}\right)$ is obtained by evaluating Equation $F .25$ for $t=t_{2}$. The value of $a_{s}(t)$ is then obtained by inserting Equation F.32 into Equation $F .31$ and solving for $a_{S}(t)$. One obtains

$$
a_{s}=1 / 2 \frac{Q_{l}}{M_{s}} \lambda_{p} a_{p o}\left(t-t_{2}\right)^{2}+\frac{Q_{\ell}}{M_{s}} a_{p}\left(t_{2}\right)\left(t-t_{2}\right)+a_{s}\left(t_{2}\right)
$$


where $a_{s}\left(t_{2}\right)$ is obtained by evaluating Equation $F .29$ for $t=t_{2}$. It is possible, by algebraic manipulations not to be presented here, to express $a_{s}(t)$ in the time interval $\left(t_{2}, t_{1}+\tau\right)$ in a form different than given in Equation F.33. This form is

$$
a_{s}=a_{s}(1)-a_{s}(2)
$$

where $a_{S}(1)(t)$ is given by Equation $F .29$ and

$$
a_{s}{ }^{(2)}=1 / 2 \frac{Q_{l}}{M_{s}}(\alpha-1) a_{p o} \lambda_{p}\left(t-t_{2}\right)^{2}
$$

Once $a_{s}(t)$ is known during the time of the LOSP event [i.e., in the inteval $\left.\left(t_{1}, t_{1}+\tau\right)\right]$ it is possible to calculate the I-131 activity released to the atmosphere during the LOSP event from

$$
A_{R}=f_{p, I} \int_{t_{1}}^{t_{1}+\tau} Q_{s t}(t) a_{s}(t) d t
$$

Here $f_{p, I}$ is the steam/water partition factor given the ratio of concentration of jodine in the steam released to the concentration of iodine in the liquid water in the steam generator. The quantity $Q_{s t}(t)$ gives the rate of release of steam (mass flow rate) from the steam generators as a function of time.

If $Q_{s t}$ is approximated as piecewise constant, then the integral in Equation F.36 can be easily calculated, since Equations F.29 and F.33 show that $a_{s}(t)$ is a polynomial in $t$. The integral in Equation $F .36$ will be explicitly evaluated here only for the special case in which $Q_{s t}$ may be approximated as equal to one constant value in the time interval $\left(t_{1}, t_{1}\right.$, $+\Delta t$ ) (i.e., during the time of the iodien spike) and by another constant 
value for $t$ in $\left(t_{1}+\Delta t, t_{1}+\tau\right)$ (i.e., after the spike in the release rate of iodine from fuel to primary coolant has ended). Let

$$
Q_{s t}=\left\{\begin{array}{l}
Q_{s t}{ }^{(1)}, t_{1} \leq t \leq t_{1}+\Delta t \\
Q_{s t}{ }^{(2)}, t_{1}+\Delta t<t<t_{1}+\tau
\end{array}\right.
$$

Define the function $G(t)$ by

$$
G(t)=\frac{1}{6} \quad \frac{Q_{\ell}}{M_{S}} \alpha a_{p o} \lambda_{p} t^{3}+\frac{1}{2} \frac{Q_{l}}{M_{S}} a_{p}\left(t_{1}\right) t^{2}+a_{s}\left(t_{1}\right) t
$$

where $a_{p}\left(t_{1}\right)$ and $a_{s}\left(t_{1}\right)$ are given by Equations $F .20$ and $F .23$, with $t$ replaced by $t_{1}$ in these equations. Then, evaluation of the integral in Equation $F .36$ yields, for $\tau<\Delta t$ :

$$
A_{R}=f_{p, I^{Q}}{ }^{(1)} G(\tau)
$$

If $\tau>\Delta t$ one obtains

$$
\begin{aligned}
A_{R} & =f_{p, I} Q_{s t}(1) \int_{t_{1}}^{t_{1}+\Delta t} a_{s}(t) d t+f_{p, I} Q_{s t}(2) \int_{t_{1}+\Delta t}^{t_{1}^{1}+\tau} a_{s}(t) d t \\
& =f_{p, I} Q_{s t}{ }^{(1)} G(\Delta t)+f_{p_{1} I} Q_{s t}(2)[G(\tau)-G(\Delta t)-H]
\end{aligned}
$$

where

$$
H=\frac{1}{6} \frac{Q_{\ell}}{M_{S}}(\alpha-1) a_{p o} \lambda_{p}(\tau-\Delta t)^{3}
$$


In obtaining Equation $F .40$, use was made of the form of $a_{S}(t)$ given in Equations $F .34$ and $F .35$, in the time interval $\left(t_{1}+\Delta t, t_{1}+\tau\right)$.

\section{F.4 Specification of Model Parameters and Distribution Functions}

The spiking Model A uses $\alpha=500$ and $\Delta t=2 \mathrm{hrs}$. It should be pointed out that the NRC iodine spiking model (as applied in licensing calculations) superimposes the effects of an iodine spike from a given transient with the maximum I-131 concentration from a previous transient. Our analysis treats this superposition more realistically since the time $t_{1}$ is treated as a random variable, and the LOSP transient is not assumed to occur at the worst possible time. A comparison of the model parameters with the data is given in Section F.2.

The equilibrium level of the primary coolant I-131 specific activity, apo, is a random variable which depends on the clad defect level $\mathrm{f}_{\mathrm{cd}}$. The value of $a_{p o}$ is proportional to the clad defect level $f_{c d}$, since values reported for $f_{c d}$ are actually inferred from measured primary coolant activity concentrations on the basis of a model which assumes proportionality (see Reference 50, page 4-1 ff). (The quantity $f_{c d}$ should more properly be called the apparent clad defect level). The distribution of clad defect levels is given in the PWR - GALE report(7). The constant of proportionality between clad defect level and equilibrium primary coolant concentration may be obtained from table 11.1-6 of RESAR-3S. (8) One obtains

$$
a_{p o}=2.33 f_{c d}
$$


where $f_{c d}$ is the clad defect level in percentage and $a_{p o}$ is the equilibrium primary coolant concentration in $\mu \mathrm{Ci} / \mathrm{gm}$. The failed fuel distribution was developed assuming a log nomal distribution with .12 percent and 1 percent designated as the failed fuel at the median and 95 percent bound respectively. Thus, for $\mathrm{f}_{\mathrm{Cd}}=.12$ percent, $\mathrm{a}_{\text {po }}=.28 \mu \mathrm{Ci} / \mathrm{gm}$.

The inagnitude $Q_{\ell}$ of the primary-to-secondary leak rate is a random variable. Table 2-13 of the PWR -GALE report, (7) which is reproduced as Table F2, gives monthly average primary-to-secondary leakages for various PWR's. The probability of a leak of any size was estimated to be 36 . We assurne that the months that the plant was shutdown can be ignored and that peak rates occurring within each month for each plant are independent. Figure 2.2 shows the calculated CCF for the maynitude of the primary to secondary leak rate based on Table F2.

The time $t_{1}$ between the last startup and the LOSP trip is also a randon variable. If reactor trips are assumed to be a Poisson process, then the probability density function for $t_{1}$ is

$$
f_{1}\left(t_{1}\right)=\lambda_{1} e^{-\lambda} t_{1}
$$

where $\lambda_{1}$ is the rate at which reactor trips occur. According to the Reactor Safety Study (1) (Appendix V, page 36) there are about a total of ten shutdowns per reactor year; one of these would be a refueling shutdown. Thus, there are some nine shutdowns per reactor year where iodine spiking would be of concern. Thus

$$
\lambda_{1}=9 / \mathrm{yr}=.001 / \mathrm{hr}
$$


TABLE F2

AVERAGE DAILY LEAK RATE DATA

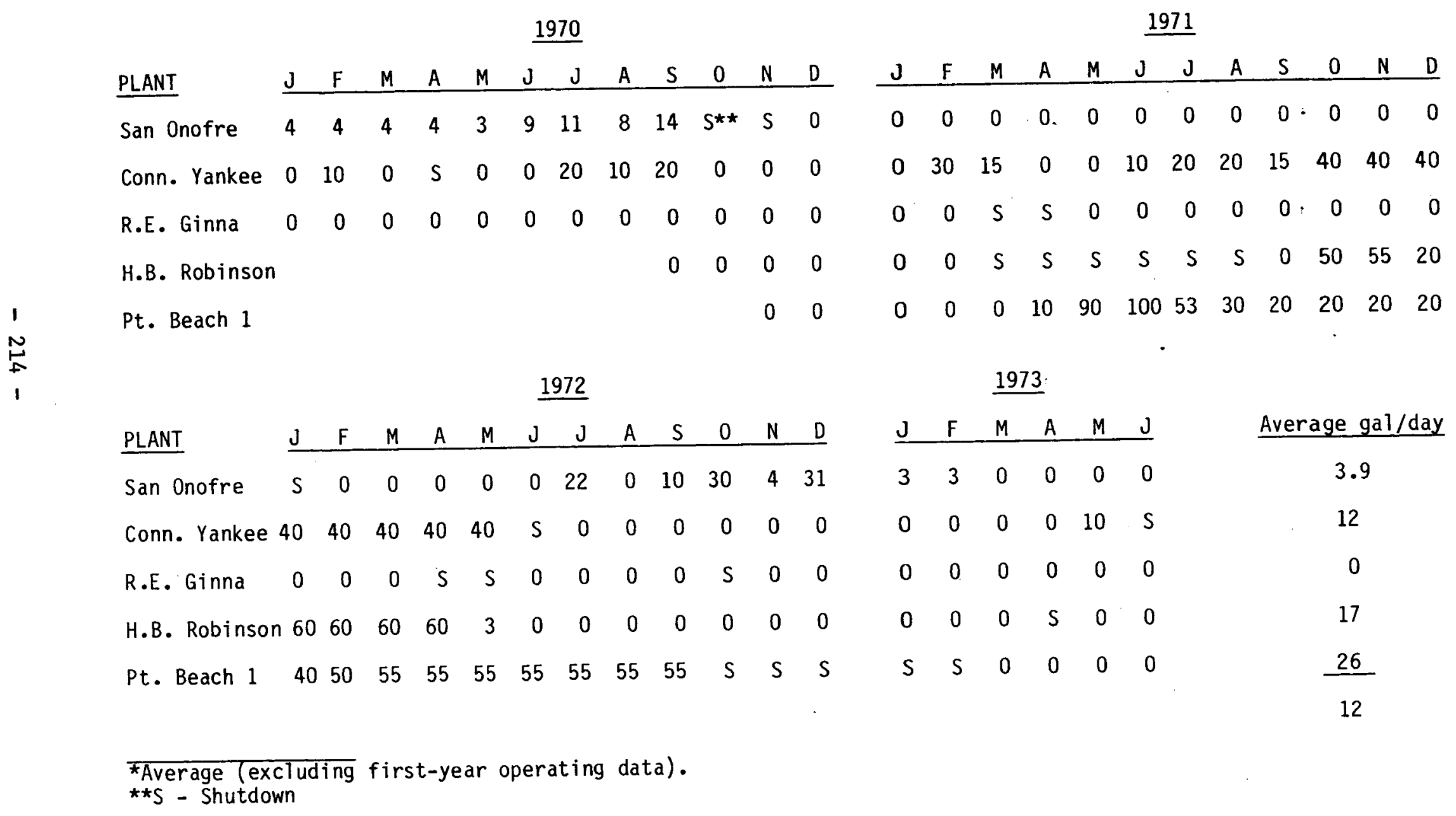


In order to obtain the probability per reactor year of a given release due to a LOSP trip, the expected frequency of LOSP trips is required. This is

$$
f_{\text {LOSP }}=.2 / y r
$$

according to Reactor Safety Study data.

The LOSP outage time $\tau$ is a random variable; the repair time distribution for LOSP is given in the Reactor Safety Study for the Bonneville'Power Administrative grid, and was used in our study. The CCF for the outage time is shown in Figure 2.3.

The steam blowdown rate versus time curve shown in Figure 2.4 was taken from Table 15.2-5 of RESAR-3S, (8) page 15.2-79. The rate at which the steam was released was developed using this blowout versus time curve. The steam blowdown rate during the first two hours was calculated to be $231,500 \mathrm{lb} / \mathrm{hr}$ and after two hours to be $182,500 \mathrm{lb} / \mathrm{hr}$.

\section{F.5 The Computer Model}

A computer model was developed that builds a cumulative frequency histogram of radioactive releases to the atmosphere. The computer model developed considers the parameters of the problem as well as the regulations placed on nomal plant operations to construct a conditional cumulative frequency histogram of radioactive releases (I-131) to the atmosphere. The continuous parameters of the problem considered are failed-fuel percentage, leak rate from primary to secondary, the time to restore off-site power, and time since last turbine trip--which is important for modeling iodine spiking. The constraints imposed on the system by NRC regulations are a primary to 
secondary system leak rate of not more than $10 \mathrm{gal} / \mathrm{min}$ and a maximum radioactivity of $9 \mathrm{Ci} \mathrm{I}-131$ in the secondary coolant loop.

The release magnitudes and their respective frequencies, representative of expected releases during normal operation due to loss of off-site power are estimated by the model using stochastic sampling of the continuous parameters while incorporating the constraints on leak rates and steam generator loop activity. The model output provides a histogram of the release magnitude versus frequency of occurrence. The probability of a release via this scenario is obtained by multiplying the probability of LOSP by the probability of a tube leak. This condition specifies an event with probability of occurrence equal to .072 . The event as described will occur approximately 72 out of every 1,000 reactor years, and will result in $I-131$ release to the atmosphere due to cracks in the heat exchangers and LOSP.

A conditional probability density function for release magnitudes was developed by many iterations of the model, each iteration selected randomly, based upon current available data, values for the following:

- failed-fuel percentage;

- leak rates from primary to secondary;

- duration of loss of off-site power;

- time since last turbine trip.

The model then calculates primary coolant activity based upon the randomly selected failed-fuel percentage, and the secondary activity based upon the randomly selected leak rate and the randomly selected failed-fuel percentage. These values were then used as input into the iodine spiking model.

The iodine spiking model calculates the number of curies of I-131 released to the atmosphere for respective inputs as previously described. The equations used are sumnarized in Table F3 and F4. On each iteration these 
release magnitudes are stored in appropriate bins. Upon completion of the number of requested samples, the relative frequencies of occurrence are calculated for each magnitude of I-131 released. This calculation divides the number of iterations into the number of events that fell into each bin representing a given range of release magnitudes. This density function has not yet considered the probability of LOSP and the probability of a leak from the primary coolant system to the secondary coolant system. This calculation requires that each cell in the Monte Carlo developed probability density function be multiplied by .072 , wich is the probability that LOSP and a leak occur simultaneously.

A flow diagram of the partial failure computer model, which includes the model inputs, the basic logic of the model and the model outputs, is shown in Figure F2. The inputs to the model are the number of samples, a distribution percentage of failed-fuel distribution, frequency of primary to secondary leak rate distribution, and duration of loss of off-site power distribution. The number of iterations was arbitrarily set at 10,000 to provide sufficient iterations to assure a smooth output function. 
0

$A_{R}=f_{p, I} \cdot Q_{\text {STEAMI }}\left[\frac{1}{6} \frac{Q_{\text {leak }}}{M_{s}} \lambda_{p}{ }^{a a_{p o}} \tau^{3}+\frac{1}{2} \frac{Q_{\text {leak }}}{M_{s}} a_{p}\left(t_{1}\right) \tau^{2}+a_{s}\left(t_{1}\right) \tau\right]$ 0 where

$A_{R} \quad=$ number of curies of $I-131$ released to the atmosphere

$f_{p, I}=I-131$ liquid to gas partition factor $=.1$

$\mathrm{Q}_{\text {STEAMI }}$

$=$ Rate of steam release for LOSP in (0-2) hours $=$

$Q_{\text {leak }}=$ Primary to secondary leak rate $=$ randomly sampled

$M_{s} \quad=$ Total mass of steam $=3.5 \times 10^{5} 1 \mathrm{bs}$

$\lambda_{p}$

$\alpha$

$=$

$=.07 / \mathrm{hr}$

$a_{\text {po }}$

= Spike factor

$=500$

= Primary activity at the time of LOSP = calculated as previously discussed

$\tau=$ Duration of loss of off-site power $=$ randomly sampled

$t_{1}=$ Time since last turbine trip $=$ randomly sampled

$$
\left(\lambda_{\text {trip }}=10^{-3} / \mathrm{hr}\right)
$$

$$
a_{p}\left(t_{1}\right)=a_{p o}+\beta a_{p o} e^{-\lambda_{p} t_{1}}
$$

where

where

$$
\begin{aligned}
& \beta=\alpha-(\alpha-1) e^{-\lambda} p \Delta t \\
& a_{s}\left(t_{1}\right)=a_{s o}+\left(a_{s i}-a_{s o}\right) e^{-\lambda_{s} t_{1}}+\frac{\beta a_{s 0} \lambda_{s}}{\lambda_{s}-\lambda_{p}}\left(e^{-\lambda_{p} t_{1}}-e^{-\lambda_{s} t_{1}}\right)
\end{aligned}
$$

$$
\begin{aligned}
a_{\text {so }}= & \begin{array}{l}
\text { Secondary coolant activity at the time of LOSP }= \\
\text { calculated as previously discussed }
\end{array} \\
a_{s i} & a_{\text {so }}\left[e^{-\lambda_{s} \Delta t}+\alpha\left(1-e^{-\lambda} s t\right)+\frac{(\alpha-1) \lambda_{s}}{\lambda_{p}-\lambda_{s}}\left(e^{-\lambda_{p} \Delta t}-e^{-\lambda} s t\right)\right. \\
\lambda_{s} & =
\end{aligned}
$$

where

$$
\Delta t \quad=2 \text { hours }
$$


TABLE F4

Iodine Spiking Model Equations, $\tau>2$ hours

$A_{R}=f_{p, I} \cdot \Omega_{\text {STEAMI }} G(2 \mathrm{hrs})+f_{p, I} Q_{\text {STEAM } 2} \quad[G(\tau)-G(2 \mathrm{hrs})]-\Delta A_{R}$

where

$A_{R} \quad=$ number of curies of $I-131$ released to the atmosphere

$f_{p, I}=I-131$ liquid to gas partition factor -.1

$Q_{\text {STEAM1 }}=$ Rate of steam release for LOSP in $(0-2)$ hours $=$

$Q_{\text {STEAM2 }}=$ Rate of steam release for LOSP in $(2-\infty)$ hours $=$

$G(\tau)=\left[\frac{1}{6} \frac{Q_{1 e a k}}{M_{s}} \lambda_{p}{ }_{p a} a_{p o} \tau^{3}+\frac{1}{2} \frac{Q_{\text {ieak }}}{M_{s}} a_{p}\left(t_{1}\right) \tau^{2}+a_{s}\left(t_{1}\right) \tau\right]$

where

$$
\begin{aligned}
& Q_{\text {leak }}=\text { Primary to secondary leak rate }=\text { randomily sampled } \\
& M_{S} \quad=\text { Total mass of steam }=3.5 \times 10^{5} \text { lbs } \\
& \lambda_{\mathrm{p}}=\quad=.07 / \mathrm{hr} \\
& \alpha \quad=\text { Spike factor } \quad 500 \\
& \mathrm{a}_{\text {po }} \quad=\text { Primary activity at the time of LOSP = calculated as } \\
& \left(\lambda_{\text {trip }}=10^{-3} / \mathrm{hr}\right) \\
& \Delta A_{R}=f_{p, I} \cdot Q_{\text {STEAM2 }} \times \frac{1}{6} \frac{Q_{\text {leak }}}{M_{s}} \lambda_{p}(\alpha-1) a_{p o}(\tau-2)^{3}
\end{aligned}
$$




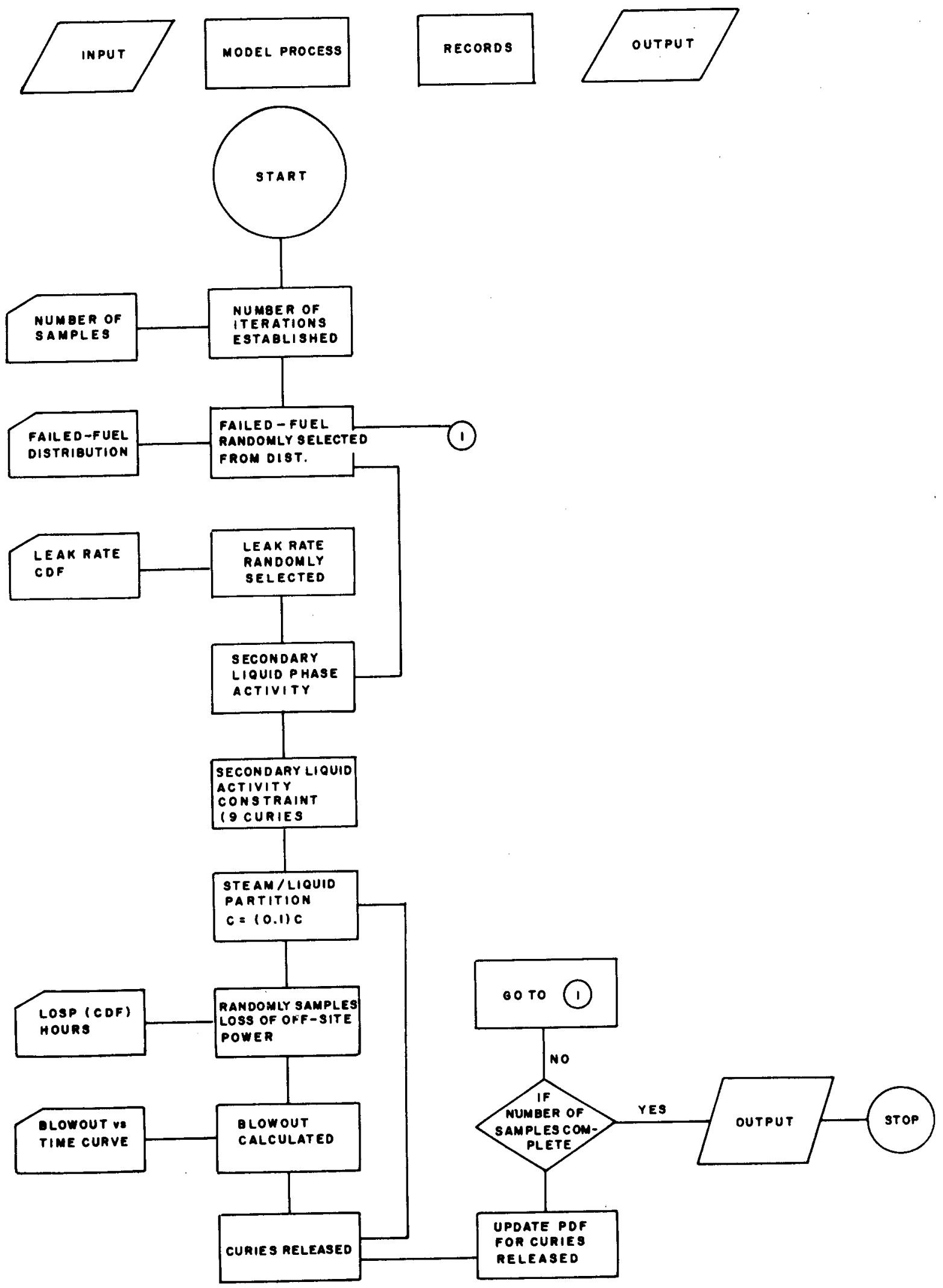

Figure F3. Flow chart for partial failure calculation of LOSP accident 
APPENDIX G

STEAM GENERATOR TUBE RUPTURE

WITH LOSS OF OFFSITE POWER

\section{G.1 Introduction}

The release of $I-131$ and Xe-133 in the SGTR with LOP sequence depends on the concentrations of these isotopes in the primary coolant. This, in turn, depends on the fraction of core power coming from fuel rods with clad defects. The radioiodine release will also depend on the "iodine spiking" phenomenon. It has been observed that the radioiodine concentration can increase markedly in the primary coolant of a pressurized water reactor after a sufficiently great power reduction. This phenomenon is described in detail in Appendix F.

Two models have been proposed for the calculation of I-131 release. In one model, the primary coolant flowing through the break is assumed to mix uniformly with the water on the secondary side of the steam generator. However, the possibility of non-uniform mixing is taken into account by using a steam/water partition factor of .1 , instead of a steam/water partition factor of about $1 / 200$, which would be the case $(34,36)$ if there were uniform mixing. This model is considered to be our best estimate model. A second, more conservative model, is also used. Postma and $\operatorname{Tam}(37)$ suggest the possibility of a mechanism for the release of most of the iodine in the primary coolant flowing through the SGTR. In this model the liquid primary coolant flowing through the SGTR atomizes into drops with a diameter of about 2 microns. These drops rise to the surface of the water within steam bubbles (with a maximum dianeter 
of $3.6 \mathrm{~cm})$. Because of the fineness of the drops they are essentially carried along with the steam and are released to the atmosphere. A second bounding calculation of the release of radioiodine frorn the steam generator was performed in which all of the iodine flowing through the SGTR is released to the atmosphere.

\section{G.2 Activity Release Calculation}

We first consider the I-131 atmospheric release as calculated by the best estimate model. Iodine spiking from the shutdown initiated by the SGTR itself is not included. The reason for this is that according to Eikelposch, Seepolt and Hock(38), a relatively small fraction of the radioiodine released from the fuel rod to the prinary coolant during iodine spiking occurs during the first hour after shutdown. (In their full paper, presented at the June 1978 Meeting of the American Nuclear Society, they estimate about $10 \mathrm{Ci}$ of I-131 released during the first hour after shutdown, from each defective rod, and 80 Ci during the first 24 hours.) We are interested in the first half-hour after shutdown, since we assume that the release from the faulty generator is terminated at the end of thirty minutes, as is consistent with the RESAR-3SA description of the accident.

Initially, when a double-ended guillotine SGTR occurs, the flow through the break is relatively large. As the pressure in the primary system is decreased, the break flow decreases, and ceases when the primary coolant pressure is equal to the pressure on the secondary side of the steam generator. According to the RESAR-3S description, the primary and secondary side pressures are equalized in the faulty steam generator at the end of thirty minutes. 
Take the origin of time $t=0$ at the time of the SGTR. The break mass flow rate $Q_{b}$ is assumed constant at $Q_{0}$ for $0<t<t_{0}$, and is then assumed to ramp down linearly to zero, reaching the value zero at $t_{1}=30 \mathrm{~min}$. The value of $Q_{0}$ used was $2,180 \mathrm{kgm} / \mathrm{min}(4800 \mathrm{lbm} / \mathrm{min})$, from Reference 39 , and the value of $t_{0}$ was chosen so that the total integrated mass flow through the break is $56,800 \mathrm{kgm}(125,000 \mathrm{lbm})$, the value given in $\operatorname{RESAR-3S}(8)$. A value of $t_{0}=22$ minutes is obtained.

The mass flow rate of $\bar{Q}_{s t}$ steam from the faulty steam generator was assumed constant over the 30 minute period, and such that the total amount of steam released over the 30 minute period is $21,300 \mathrm{kgm}(47,000 \mathrm{lbm})$, the RESAR-3S value.

The I-131 activity released from the faulty steam generator is given. by

$$
A_{R}=f_{p, I} \bar{Q}_{s t} \int_{0}^{t} a_{s}\left(t^{\prime}\right) d t^{\prime}
$$

where

$$
\begin{aligned}
& f_{p, I}=\begin{array}{c}
\text { steam/water partition factor for radioiodine in the faulty steam } \\
\text { generator. }
\end{array} \\
& a_{s}(t)=\begin{array}{c}
\text { specific activity of radioiodine in the water on the secondary side } \\
\text { of the faulty steam generator. }
\end{array}
\end{aligned}
$$

The quantity $a_{s}(t)$ is computed from

$$
a_{s}(t)=\frac{\bar{a}_{p}}{M_{s}} \quad \int_{0}^{t} Q_{b}\left(t^{\prime}\right) d t^{\prime}+a_{s o}
$$


where $a_{\text {so }}$ is the specific activity in the secondary side water of the faulty steam generator at the start of the accident; $M_{S}$ is the water mass (assumed time independent) on the secondary side of the faulty steam generator, and $a_{p}$ is the primary coolant specific activity (of I-131), assumed constant throughout the thirty minutes duration of the release. Equation G2 assumes that all the activity which pours into the secondary side through the break mixes unifomly with the secondary side water, and neglects the radioiodine removal processes from the secondary side in computing the buildup of secondary side activity. This is a good approximation since the half-life of I-131 is 8.05 days, so that removal by radioactive decay is negligible. Moreover, removal by release through the safety valves does not appreciably affect the radioiodine buildup on the secondary side of the faulty steam generator since only a small fraction of the radioiodine in the faulty steam generator is released through the safety valves.

The specific activity of I-131 in the primary coolant, $\bar{a} \rho$, depends on the clad defect level and on the time after the last reactor startup, because of the iodine spiking phenomenon. An expression for $\bar{a}_{p}$ is developed in Sections $F 2$ and $F 3$, we quote the resulting Equation F20

$$
a_{p}=a_{p o}\left(1+\beta e^{-\lambda \tau}\right)
$$

where

$$
\begin{aligned}
\tau & =\text { time interval from the last startup to the SGTR } \\
a_{\text {po }} & =\text { equilibrium I-131 concentration in primary coolant } \\
\lambda & =.07 \mathrm{hr}^{-1} \text { total I removal rate } \\
\beta & =65 \mathrm{I} \text { spiking factor }
\end{aligned}
$$


After performing the two integrations in Equations G1 and G2, the estimated I-131 released from the steam generator is

$$
A_{R}=f_{p, I} \bar{Q}_{s t} \bar{a}_{p} \frac{Q_{0}}{\bar{M}_{s}}\left[\frac{1}{2} t_{1}{ }^{2}-\frac{1}{\bar{b}}\left(t_{1}-t_{0}\right)^{2}\right]
$$

For the more conservative model in which essentially all of the I-131 activity in the primary coolant flowing through the ruptured steam generator tube is released to the atmosphere,

$$
A_{R}=\bar{a}_{p} \int_{0}^{t} Q_{b}(t) d t
$$

For Xe-133, the model is very simple; the primary coolant specific activity for Xe-133 is its equilibrium value and is proportional to the clad defect level. All of the Xe-133 flowing through the rupture in the steam generator tube is released to the atmosphere.

\subsection{Probability Distribution Function for the Activity Releases}

The I-131 activity release depends on $\tau$, the time interval between the last start up and the SGTR and also through $a_{p o}$ on the clad defect level. Equations G3 and G4 or G5 can be combined to show this dependence explicitly:

$$
A_{R}=k f_{c d} z
$$

where $k$ depends on which of the two models is used for estimating the releases; $f_{c d}$ is the clad defect level, and

$$
z=1+\beta e^{-\lambda} T^{\tau}
$$


Both the quantities $f_{c d}$ and $z$ are random variables. It is convenient to consider the quantity $A^{\prime}=f_{c d}$. The probability density function (pdf) for $A^{\prime}, g_{A^{\prime}}\left(A^{\prime}\right)$, can be written in terms of the pdf for $f_{c d}, g_{c d}\left(f_{c d}\right)$, and the pdf for $z, g_{z}(z)$ in the form

$$
g_{A^{\prime}}\left(A^{\prime}\right)=\int_{1}^{1+\beta} g_{z}(z) g_{c d} \frac{A^{\prime}}{z} \frac{1}{z} d z
$$

Once $g_{A}{ }^{\prime}\left(A^{\prime}\right)$ is obtained, the complementary cumulative distribution function for $A^{\prime}$ is obtained by numerical integration. The complementary distribution for $A$ is then obtained easily, since $A=k A^{\prime}$.

The pdf for $f_{c d}$ was obtained from data given in the PWR-GALE report $(7)$. Table 2-10 of this report gives the clad defect level and associated burnup times for various PWRs. Fram these data, an approximate, piecewise constant, pdf was obtained. This pdf is:

$$
\begin{aligned}
& \text { For } 0 \leq f_{c d}<.035, g_{c d}=11.06 \\
& \text { For } .035 \leq f_{c d}<.065, g_{c d}=4.08 \\
& \text { For } .065 \leq f_{c d}<.125, g_{c d}=3.4 \\
& \text { For } .125 \leq f_{c d}<.245, g_{c d}=.875 \\
& \text { For . } 245 \leq f_{c d}<.515, g_{c d}=.344 \\
& \text { For . } 515 \leq f_{c d}<.755, g_{c d}=.275
\end{aligned}
$$

The pdf for $z$ can be obtained from the pdf for $\tau$, since $z$ is a function of $\tau$, as given by Equation G7. The quantity $\tau$ is the time between the SGTR and the last startup, and is assumed to be exponentially distributed. Nine reactor trips per year which can lead to iodine spiking are assumed. Then the pdf for $\tau$ is

$$
g_{\tau}(\tau)=\lambda_{s} e^{-\lambda} \tau
$$


where $\lambda_{s}$ is the mean time between trips $\left(\lambda_{s} \approx .001 / \mathrm{hr}\right)$. It follows that

$$
g_{z}(z)=g_{\tau}(\tau) \frac{d \tau}{d z}=\frac{{ }^{\lambda}}{\lambda_{T} \beta}\left(\frac{\beta}{z-1}\right)^{\gamma}
$$

where $\gamma=\left(\lambda_{T}-\lambda_{S}\right) / \lambda_{T}$

The distribution function calculation for the Xe-133 release-is simpler, since the Xe-133 release is simply proportional to the clad defect level. Denote the constant of proportionality by kxe so that

$$
A_{R}^{X e}=k_{X e}^{f} c d
$$

The

$$
\operatorname{pr}\left\{A_{R}^{X e}>A_{0}\right\}=\operatorname{pr}\left\{f_{c d}>\frac{A_{0}}{k_{X_{e}}}\right\}
$$


APPENDIX H

CORE BARREL DROP ANALYSIS

The following provides the details to our analysis of the probability and consequences of a Core Barrel Drop into an open reactor vessel.

According to Reference 9, dropping the upper core barrel assembly into an open reactor vessel can lead to some fuel rod failures. Taking an upper bound estimate of $1 / 2$ hour of crane operation per refueling during which the accident might take place, and a probability of $3 \times 10^{-6} / \mathrm{hr}$ for crane failures (see reference 45 for crane failure probability) one obtains a probability of $2 \times$ $10^{-6} / \mathrm{yr}$ for dropping the upper core barrel into the open reactor vessel.

The estimate of the probability of a core barrel drop may have to be refined later. It should be noted that at the Robert E. Ginna Unit 1 reactor, the reactor core barrel and internals were dropped 6 feet onto a storage rack as a result of failure of the crane's electromagnetic brake; the mechanical brake was not installed at the time of the occurrence. The incident occurred on July 18, 1969, before initial criticality. In the light of this incident, it may be optimistic to assume a probability of $2 \times 10^{-6}$ per refueling for a core barrel drop into the reactor vessel.

For orientation purposes, let us assume that in this accident the fuel rods in one assembly (204 rods) fail and release their activity to the reactor vessel water. Let us further assume that:

(1) The inventory of I-131 in the reactor is . $85 \times 10^{8} \mathrm{Ci}$.

(2) The accident occurred in an assembly with a radial power factor of unity. 
(3) The accident occurred 90 hours after shutdown; this is roughly the time at which the upper core support is removed from the reactor vessel.

(4) About $5 \%$ of the I-131 core inventory is in the fuel rod gap region. This is the release fraction given in Table VII-1 of reference 45.

The I-131 inventory in (1) above is valid for a typical four-loop westinghouse PWR with a power rating of 3200 MWt. The value came from Table VI 3-1 of the Reactor Safety Study. (1)

There are 193 assemblies in a typical 4 loop Westinghouse PWR. The I-131 activity in the gap region of the fuel rods in an assembly with a radial peaking factor of unity is therefore:

$G=.05 \times .85 \times 10^{8} / 193 \mathrm{Ci}=2.2 \times 10^{4} \mathrm{Ci}$.

The half life of I-131 is 8.05 days. Hence, 90 hours after shutdown the I-131 activity has decayed by a factor

$$
\exp (-\lambda t)=\exp -(\text { In 2) }(90) /(8.05 \times 24)=.724
$$

Thus, ninety hours after shutdown the gap activity in the 204 rods of one assembly is

$$
G^{\prime}=e^{-\lambda t_{G}}=1.6 \times 10^{4} \mathrm{Ci}
$$

Not all of the I-131 activity in the gap is in vapor form; only about. $2 \%$ would be in vapor fom at refueling temperatures. Moreover, all of the gap activity released to the reactor vessel water is not released to the containment atmosphere. Reference 40 states that there is experimental evidence of a decontamination factor of 1000 for the reactor vessel water. Hence the release to the containment atiliosphere is

$$
.02 \times 10^{-3} \times G^{\prime}=.32 \mathrm{Ci}
$$

The release from the containment depends on whether the containment isolates, and on how much activity is released before containnent isolation occurs. It also depends on whether the contaiment exhaust is filtered.

A docurrent was mailed anonymously to the Union of Concerned Scientists and 
included as an enclosure in a letter, (41) from the U.S. Nuclear Regulatory Commission to various utilities; the letter requested an analys is of a fuel handling accident within contaimment. This document states in part; "W is not aware of the NRC bases for not addressing a fuel handling accident inside contaiment, the bases may include:

(1) The assumption that the containment will be isolated during refueling operations;

(2) that the contaiment could be isolated quickly enough to limit offsite consequences; or

(3) that filtration capability comparable to that in fuel storage building exhausts exists in the containment purge exhaust.

Therefore we conservatively assumed that the entire 0.32 curies of I-131 would be released to the environment.

The other iodine isotopes were similarily treated to obtain the curie quantities in which they were released. They resulted in negligible contributions as compared to the I-131.

The noble gas releases from the core barrel drop accident was also calculated via the same method. The entire yap inventory of the noble gases is released to the environment, with no reduction taken for decontamination factors or liquid fractions.

The noble gase releases proved to be the primary weighted risk to the public in this accident. 
APPENDIX I

SPENT FUEL HANDLING ACCIDENT IN

THE SPENT FUEL STORAGE POOL

\section{I.1 Uncertainty Analysis}

To provide an estimate of the accuracy of the calculated distribution function, an uncertainty analysis was performed for the iodine releases. The iodine release was chosen for this because of the much greater uncertainty relative to the noble yas releases.

In this uncertainty analysis some of the parameters describing the various probability distributions entering into the analys is were themselves treated as random variables. The probability distribution for these parameters were chosen for the most part in a subjective fashion; the uncertainties obtained are best interpreted in a Bayesian sense.

The quantity $N_{r}$ has the probability distribution

$$
\begin{aligned}
& \operatorname{pr}\left\{N_{r}=15 \mid \alpha=1\right\}=P_{1} \\
& \operatorname{pr}\left\{N_{r}=204 \mid \alpha=1\right\}=1-p_{1}
\end{aligned}
$$

where $p_{1}$ is uniformly distributed between.2 and .8. (For the base case, $\left.p_{1}=.5\right)$.

The distance of release $H$ below the surface of the SFSP water is 26 feet or 40 feet with probability $p_{2}$ and $1-p_{2}$ respectively, where $p_{2}$ is uniformly distributed between .25 and .75. (For the base case, $p_{2}=.5$ ).

The probability the auxiliary building filter is failed (i.e., $\operatorname{pr}\{x i n=1\}$ is $10.5-p_{4}$ where $p_{4}$ is uniformly distributed between 2 and 3 . (For the base case, $p_{4}=2.5$ ). 
The probability P5 that at least one rod fails, given that a fuel handling accident has occurred, is distributed with the probability density function (p.d.f.) given by

$$
f\left(p_{5} / 0\right)=7\left(1-p_{5}\right)^{6}
$$

This probability density function is derived as follows:

Let $p$ denote the probability that a fuel assembly drop in the spent fuel pool results in at least one rod failure. Then, since no information is available relating to this probability, we take its "a priori" distribution function, $h(p)$, to be uniform in the range $0 \leq p \leq 1$. The conditional probability, $g(0 / p)$, that no rod fails in six drops, given $p$, is

$$
g(0 / p)=(1-p)^{6}
$$

The Bayesian formula

$$
f(p / 0)=\frac{g(0 / p) h(p)}{\int_{0}^{1} g(0 / p) h(p) d p}
$$

then yeilds for the "a posteriori" distribtuion of $p$, given no failures in six drops,

$$
f(p / 0)=\frac{(1-p)^{6}}{\int_{0}^{1}(1-p)^{6} d p}=7(1-p)^{6}
$$

since $h(p)=$ const. The expectation value of $p$ is found by straightforward integration:

$$
E(p)=\int_{0}^{1} p f(p / 0) d p=7 \int_{0}^{1} p(1-p)^{6} d p=0.125
$$


The RADREL code calculates the complementary cumulative distribution functions CCF for the I-131 organic and inorganic activity releases by the Monte Carlo method, given that a fuel handling accident with at least one rod failure has occurred. The results are then multiplied by the probability per reactor-year that an accidental drop will occur and then by the probability that, if a fuel handling accident has occurred, at least one rod fails. This yeilds the probability per reactor-year of a release exceeding a given number of Curies.

Each Monte Carlo run was divided into 30 experiments each consisting of 1000 histories (trials), so that there were 30,000 histories in all. There were 30 output bins for the number of I-131 Curies released, the upper and lower $l$ imits of the $i$ th bin being $10(i / 2-10) C i$ and $10(i / 2-9.5) \mathrm{Ci}$, respectively. For each history both the organic I-131 release and the inorganic I-131 release were calculated.

The Monte Carlo calculation of the distribution function for I-131 release, given a fuel handling accident with cladding failure has occurred, was for the most part a direct simulation of the accident, where, for each random variable, sampling fron the appropriate distribution was performed. However, in order to reduce the statistical error from the finite number of histories used, two non-analogue Monte Carlo devices were used. The first was stratified sampling on the variable $N_{R}$. Instead of picking the values $N_{R}=15$ and $N_{R}=204$ with the appropriate probabilities on each trial, the Monte Carlo run was arranged so that (for the base case where $\operatorname{pr}\left\{N_{R}=15 \mid \alpha=1\right\}$ $=.5$ ) exactly half of the trial (in each experiment) had $N_{R}=15$ and the other half had $N_{R}=204$. 
The second Monte Carlo device used was associated with the auxiliary building filter. Each history has associated with it two inorganic Curie releases and two organic Curie releases. One of the two inorganic Curie releases corresponds to the release that would occur if the auxiliary building filter is failed. The counts in the appropriate bin on each history is augmented not by unity but by 1-PAF, where PAF is the probability the auxiliary building filter is not failed. The other inorganic release (for each history) corresponded to the release if the auxiliary building filter operates. The counts in the appropriate bin are here augmented by PAF. The method may be viewed as breaking up $f(C)$, the probability density for I-131 releases (say inorganic release to be specific) into

$$
\begin{aligned}
f(C)= & f(C \mid \text { aux bldg filter fails })\left(1-P_{A F}\right) \\
& +f(C \mid \text { aux bldg filter works }) \text { PAF }
\end{aligned}
$$

and estimating both of the conditional density functions on the right hand side of this equation by the same Monte Carlo histories.

As mentioned earlier, each Monte Carlo run was broken up into 30 experiments. The reason for this was to assess the statistical error arising from the use of the finite number of histories. By seeing how much the counts in each bin fluctuate from one experiment to another an estimate of the statistical error can be obtained. The estimates of the probability density function (p.d.f.) obtained from each of the 30 experiments can be combined to give an overall estimate. Statistical errors both in the p.d.f. and the complementory c.d.f. were found. The precise procedure was as follows.

In the case of each of the 30 experiments, the total number of counts in each bin and its square were found. These were combined to give the total 
counts in each bin and its variance. In addition, the sample estimate of probability of obtaining a value in a particular bin as well as its relative variance was detennined, this producing the probability density function (p.d.f.) in histogram form. The CCF of the Curies released for each experiment was obtained by summing the probabilities in the appropriate bins. The corresponding quantity for the 30 experiments was found by averaging. Finally, the relative variance of the CCF found. The formulas used in the above statistical analysis follows.

Let $x_{j i}$ denote the number of counts corresponding to a given bin in the $i^{\text {th }}$ history of the $j^{\text {th }}$ experifient. Then the total number of counts in the jth experiment, $y_{j}$, is

$$
y_{j}=\sum_{i=1}^{1000} x_{j i}
$$

while the total number of counts in 30 experiments, $z$, is

$$
z=\sum_{j=1}^{30} y_{j}
$$

The variance of $z$, from C.9, is yiven by

$$
\begin{aligned}
\operatorname{var}(z) & =\sum_{j=1}^{30} \operatorname{var}\left(y_{j}\right) \\
& \approx \sum_{j=1}^{30} \operatorname{svar}\left(y_{j}\right)
\end{aligned}
$$

where svar $\left(y_{j}\right)$ denotes the sample variance of $y_{j}$ as obtained from the 30 experiments. We have, by definition,

$$
\operatorname{svar}\left(y_{j}\right)=\frac{\sum_{i=1}^{30}\left(y_{i}-\bar{y}\right)^{2}}{29}
$$

where

$$
\bar{y}=\frac{\sum_{1}^{30} y_{i}}{30}
$$


Substituting I.12 into I.11, we get

$$
\operatorname{svar}\left(y_{j}\right)=\frac{30 \sum_{i=1}^{30} y_{i}{ }^{2}-\left(\sum_{i=1}^{30} y_{i}\right)^{2}}{30 \times 29}
$$

which is independent of $j$.

Hence, from I.10 and I.13, for the standard deviation $\operatorname{std}(z)$

$$
\begin{aligned}
\operatorname{std}(z) & =\sqrt{\operatorname{var}(z)} \\
& =\sqrt{30 \operatorname{var}\left(y_{j}\right)} \\
& =\sqrt{\frac{30 \sum_{1}^{30} y_{i}^{2}-\sum_{1}^{30} y_{i}^{2}}{29}}
\end{aligned}
$$

To convert $z$ and $\operatorname{std}(z)$ to apply to estimated probabilities we need only divide by 30,000 , the total number of histories in the 30 experiments. The relative standard deviation $\operatorname{rstd}(z)$ is simply

$$
\operatorname{rstd}(z)=\frac{\operatorname{std}(z)}{z}
$$

provided $z \neq 0$. If $z=0$, we take $\operatorname{rstd}(z)=0$.

All of the above analysis refers to any one particular bin. To find the complementary cumulative distribution, we sum the counts in bin $\# i \quad(i=1, \ldots, 30)$ to bin \#30 in each experiment, thereby obtaining the counts in the range $10(i / 2-10)$ to $10^{5.5}$ curies for each experiment. (The upper limit of this range, $10^{5.5}$ curies, is effectively infinite, since the maximum possible release is less than $10^{5.5}$ curies). Summing the count in this range over the 30 experiments yields the total number of counts in the range $10(i / 2-10)$ to infinity. Dividing by 30,000 then gives the probability that the release 
exceeds $10(i / 2-10)$. The relative standard deviation of this quantity is found from formulas analogous to I.14 and I.15.

In order to perform the uncertainty analysis, the parameters describing the probability distribution functions were themselves treated as random variables, as discussed earlier. Five hundred RADREL runs using random values of the parameters were performed. The five hundred values of the CCF corresponding to a particular radioactivity release were then ordered From this, the $50 \%$ confidence limits were found.

\section{I.2 Noble Gas Release Calculations}

The noble gas release from this accident was calculated to obtain a more complete estinate of the total risk than obtained from the analys is of only halogen releases. From the analys is of the core barrel drop accident it was clear that the Xe-133 isotope was the major contributor to the dose to the public.

The $\mathrm{Xe-133}$ gap inventory in the 15 Mwt and the 20 Mwit fuel assembly rods was calculated to be 42.5 and 138.0 Curies respectively. To simplify calculations made by hand, it was assumed that the probability of handling a high power assembly was 0.5 at all times in the 100 to 200 hour refueling interval.

The probability density function describing the number of fuel rods that fail and the decay time to handling were used as described in the iodine release calculation. A decontamination factor of 1.0 was used for $\mathrm{Xe}-133$.

Table I-1 shows the results of this noble gas release calculation.

After weighting the noble gas release, and the iodine releases (both inorganic and organic) it is seen that the noble gas dominates the risk to the public. 


\section{TABLE I1}

PROBABILITY OF EXCEEDING $X$ CURIES OF $X e-133$

RELEASED IN A FUEL HANDLING ACCIDENT IN THE SPENT FUEL POOL

$\begin{array}{cc}\begin{array}{c}\text { Probability } \\ \text { (per reactor year) }\end{array} & \begin{array}{r}\text { Quantity Relea } \\ (X \subset i)\end{array} \\ 2.5(-3) & 1.0(2) \\ 1.9(-3) & 2.0(2) \\ 1.9(-3) & 3.0(2) \\ 1.4(-3) & 5.0(2) \\ 1.25(-3) & 7.5(2) \\ 1.25(-3) & 1.0(3) \\ 8.13(-4) & 2.0(3) \\ 6.25(-4) & 3.0(3) \\ 6.25(-6) & 6.0(3)\end{array}$


APPENDIX J

SPENT FUEL POOL LOSS OF

COOLING ACCIDENT ANALYSIS

\section{J.1 Engineering Physics Calculations}

In the event of a loss-of-cooling incident in the spent fuel.storage pool and subsequent failure to identify the problem or inability to correct the problem in required time period, it is possible for boiling to be initiated in the pool due to the heat load fran the spent fuel elements stored in the pool. In order to analyze the consequences of this incident, the values of several parameters of major importance must be established. These include the heat loading to the pool, the iodine release from the stored fuel pins during pool boiling, the decontamination factor for the pool during boiling, and filter efficiencies for radioiodine removal under high humidity conditions. The methods presented here provide a general neans for establishing typical values for these parameters over a range of expected operating conditions.

The heat loading to the pool is the decay heat generated by the spent fuel stored in the pool. The fission product decay heat is related to the operating power of the fuel while it was in the reactor. The decay heat release rate after shutdown is typically expressed as a fraction of the steady operating power, $P_{0}$, prior to shutdown. For the hypothetical case of infinite reactor operating time, the fraction of operating power, $P / P_{0}$, attributable to fission products decay power is given by 


$$
P / P_{0}\left(\infty, t_{s}\right)=A\left(t_{s}\right) t_{s}^{-a}
$$

where $P=$ power at time $t_{S}$

$P_{0}=$ steady operating power prior to shutdown

$t_{0}=$ reactor operating tine prior to shutdown, seconds

$t_{s}=$ cooliny time, seconds

$A$ and a are given in Table $\mathrm{Jl}$.

To account for a finite reactor operating time $\left(t_{0}<\infty\right)$, the fraction of operating power due to fission product decay is given by the following correction to the infinite time operation curve.

$$
P / P_{0}\left(t_{0}, t_{s}\right)=P / P_{0}\left(\infty, t_{s}\right)-P / P_{0}\left(\infty, t_{0}+t_{s}\right)
$$

or

$$
P / P_{0}\left(t_{0}, t_{s}\right)=A\left(t_{s}\right) t_{s}^{-a\left(t_{s}\right)}-A\left(t_{0}+t_{s}\right)\left(t_{0}+t_{s}\right)^{-a\left(t_{0}+t_{s}\right)}
$$

where $t_{0}=$ reactor operating time prior to shut down, sec. and $A\left(t_{s}\right), A\left(t_{0}+t_{s}\right)$ and $a\left(t_{s}\right), a\left(t_{0}+t_{s}\right)$ are determined from Table J1.

The above analytical expressions represent the ANS draft Standard(42) for decay energy release rates following reactor shutdown.

For $n$-core sections stored in the spent fuel pool, each having an operating power $P_{0 i}$ when in the core, an operating period in the core of $t_{0 i}$ and $a$ cooling time in the SFSP of $t_{S i}$, the total heat load to the pool, $P$, is given, by 
TABLE J 1

$\frac{t_{s}, \text { Time Interval }(\mathrm{sec})}{10^{-1}-10^{1}}$
$10^{1}-1.5 \times 10^{2}$
$1.5 \times 10^{2}-4 \times 10^{6}$
$4 \times 10^{6}-2 \times 10^{8}$

$\begin{array}{ll}\frac{\mathrm{A}}{\mathrm{a}} & \frac{\mathrm{a}}{} \\ 0.0603 & 0.0639 \\ 0.0766 & 0.181 \\ 0.130 & 0.283 \\ 0.266 & 0.335\end{array}$

TABLE J2

POOL HEAT LOAD SUMMARY

\begin{tabular}{|c|c|c|c|c|}
\hline Core Section $i$ & $t_{s i}(\mathrm{sec})$ & $\left(t_{0 i}+t_{s i}\right)(\mathrm{sec})$ & $\mathrm{P}_{0 i}(\mathrm{Btu} / \mathrm{hr})$ & $P_{i}(B t u / h r)$ \\
\hline $\begin{array}{l}1 \\
2 \\
3 \\
4 \\
5 \\
6 \\
7 \\
8 \\
9\end{array}$ & $\begin{array}{r}6.05(5) \\
1.58(7) \\
3.16(7) \\
4.74(7) \\
6.32(7) \\
7.90(7) \\
9.48(7) \\
11.06(7) \\
12.64(7)\end{array}$ & $\begin{array}{r}9.52(7) \\
11.06(7) \\
12.64(7) \\
14.22(7) \\
15.8(7) \\
17.38(7) \\
18.96(7) \\
20.54(7) \\
22.12(7)\end{array}$ & $\begin{array}{l}2.78(9) \\
2.78(9) \\
2.78(9) \\
2.78(9) \\
2.78(9) \\
2.78(9) \\
2.78(9) \\
2.78(9) \\
2.78(9)\end{array}$ & $\begin{array}{l}6.78(6) \\
1.37(6) \\
0.84(6) \\
0.61(6) \\
0.48(6) \\
0.39(6) \\
0.33(6) \\
0.28(6) \\
0.24(6)\end{array}$ \\
\hline Total 3 cores & & & & $1.32(6)=P$ \\
\hline
\end{tabular}




$$
\begin{aligned}
P= & \sum_{i=1}^{n} P_{i}=\sum_{i=1}^{n} P_{o i} A\left(t_{s i}\right) t_{s i}-a\left(t_{s i}\right) \\
& -A\left(t_{o i}+t_{s i}\right)\left(t_{o i}+t_{s i}\right)^{-a\left(t_{o i}+t_{s i}\right)}
\end{aligned}
$$

where $P=$ pool heat load, Btu/hr

$$
\mathrm{P}_{j}=\text { power of the } i \text {-th core section in the pool, Btu/hr }
$$$$
P_{0 i}=\text { operating power of the } i-t h \text { core section, Btu/hr }
$$$$
\mathrm{n}=\text { number of core sections in the pool }
$$

$t_{o i}=$ reactor operating $t$ ime for the $i$-th core section, sec.

$t_{s i}=$ cooling time interval for the $i$-th core section, sec.

$$
\begin{aligned}
& A\left(t_{s i}\right), A\left(t_{s i}+t_{o i}\right)= \text { coefficients from Table } J 1 \text { at cooling times } \\
& t_{s i} \text { and } t_{0 i}+t_{s i} \text { respectively } \\
& a\left(t_{s i}\right), a\left(t_{s i}+t_{o i}\right)= \begin{array}{l}
\text { exponents from Table } J 1 \text { at cooling times } \\
\end{array} \\
& t_{s i} \text { and } t_{0 i}+t_{s i} \text { respectively. }
\end{aligned}
$$

To obtain an estimate of the Surry pool heat loading, the following assumptions have been made:

(1) $1 / 3$ of a core is placed in the pool at each refueling and the two surry reactors are alternately refueled on a six month schedule.

(2) Each core section placed in the pool had an operating period of three years. $\left(t_{0 i}=9.46 \times 10^{7} \mathrm{sec}\right)$

(3) The total core operating power was 2441 MWT. One-third of the core generates $1 / 3$ of the power. $\mathrm{P}_{\mathrm{oi}}=2.78 \times 10^{9} \mathrm{Btu} / \mathrm{hr}$ 
(4) The loss of pool cooling occurs seven days after the last refueling shutdown.

(5) The pool holds three full cores or nine 1/3-core sections. The Surry. pool holds 464 fuel assemblies per the FSAR. This is approximately three cores (157 assemblies per core).

(6) Since $\left(t_{0 i}+t_{s i}\right)$ is always greater then $4 \times 10^{6} \mathrm{sec}, A\left(t_{0 i}+\right.$ $\left.t_{s i}\right)=0.266$ and $a\left(t_{o i}+t_{s i}\right)=0.335$, for all $i$.

Table $\mathrm{J} 2$ summarizes the contribution of each core section to the pool heat load.

The pool heatup rate, $\Delta T$, is given by

$$
\Delta T=P / C_{P} V
$$

$$
\begin{aligned}
\Delta^{T} & =\text { pool heatup rate, } \mathrm{F} / \mathrm{hr} \\
P & =\text { pool heat load, Btu/hr } \\
C_{p} & =\text { specific heat of water, Btu } / 1 \mathrm{~b}_{\mathrm{m}}-\mathrm{F} \\
\rho & =\text { pool density, } 1 \mathrm{~b}_{\mathrm{m}} / \mathrm{ft}^{3} \\
V & =\text { pool volume, } \mathrm{ft}^{3}
\end{aligned}
$$

This assumes no heat loss from the pool, which maximizes the heatup rate.

The time to heat the pool from any temperature, $T$, to $212^{\circ} \mathrm{F}$, is given by

$$
\Delta t=C_{p} V(212-T) / P
$$

where $\Delta t=$ pool heatup time, $h r$.

For pool water, $C_{p}=1 B t u / 1 b_{m}-F$. Over the range of pool temperatures anticipated in a boiling incident, 70 to $212^{\circ} \mathrm{F}$, the density varies from 62.3 to $59.8 \mathrm{lbm} / \mathrm{ft}^{3}$. Assume an average density of $60.91 \mathrm{~b}_{\mathrm{m}} / \mathrm{ft}^{3}$. 
The initial or ambient pool temperature, $T$, must be known to estimate the time required to heat the pool to $212^{\circ} \mathrm{F}$. The design basis for the fuel pit cooling system is to maintain the fuel pit water below $140^{\circ} \mathrm{F}$ when $1 / 3$ of the core is placed in the pit 150 hours after shutdown and to maintain the fuel pit temperature below $170^{\circ} \mathrm{F}$ when $1-2 / 3$ cores are placed in the pit 150 hours after shutdown (Surry SAR p. 9.5-1). The fuel pit coolers(43) have a design duty of $34.75 \times 10^{6} \mathrm{Btu} / \mathrm{hr}$ each (Surry SAR p. 9.5-3).

Johnson $(43)$ cities pool temperatures in the range of 70 to $120^{\circ} \mathrm{F}$.

The Surry spent fuel pool is approximately $76000 \mathrm{ft}^{3}$. This volume is decreased by the fuel in the pool and the storage racks. The volume associated with the fuel assemblies is estimated as follows. From the Surry SAR ( $p$. $3.32-3)$

$$
\begin{array}{lr}
\text { Fuel Weight }\left(\mathrm{UO}_{2}\right) & 175,600 \mathrm{lb}_{\mathrm{m}} \\
\text { Zircaloy Weight } & 36,3001 \mathrm{~b}_{\mathrm{m}} \\
\text { Total Weight Fuel Assemblies } & 226,200 \quad 1 \mathrm{~b}_{\mathrm{m}} \quad \text { p. } 3.5-2
\end{array}
$$

Assume the difference in weight is for the stainless steel in the core. Assume the fuel density is 91 percent of the theoretical density. The volume of $\mathrm{U0}_{2}$, Zircaloy, and stainless steel are obtained by dividing the weights by densities.

$$
\begin{aligned}
& \mathrm{uO}_{2} \text { Volume }=175,6001 \mathrm{~b}_{\mathrm{m}} / .91 \times 6851 \mathrm{~b}_{\mathrm{m}} / \mathrm{ft}^{3}=282 \mathrm{ft}^{3} \\
& \mathrm{Zr} \text { Volume }=36,3001 \mathrm{~b}_{\mathrm{m}} / 4101 \mathrm{~b}_{\mathrm{m}} / \mathrm{ft}^{3}=89 \mathrm{ft}^{3} \\
& \text { ss Volume }=14,3001 \mathrm{~b}_{\mathrm{m}} / 4901 \mathrm{~b}_{\mathrm{m}} / \mathrm{ft}^{3}=30 \mathrm{ft}^{3}
\end{aligned}
$$

Total Volume per core $=401 \mathrm{ft}^{3}$ 
For three cores the volume displaced is $1200 \mathrm{ft}^{3}$. Assume the racks displace an equivalent volume. The spent fuel pool water volume is estimated to be $76,000 \mathrm{ft}^{3}$

$-1,200 \mathrm{ft}^{3}$ fuel volume $-1,200 \mathrm{ft}^{3}$ storage rack volume

$$
73,600 \mathrm{ft}^{3}
$$

In estimating the tine for the pool to heat up to $212^{\circ} \mathrm{F}$, we can consider a range of initial pool temperatures between 70 and $120^{\circ} \mathrm{F}$ and a pool volume of $73,600 \mathrm{ft}^{3}$. Using the pool heat loading of $11.3 \times 10^{6} \mathrm{Btu} / \mathrm{hr}$ estimated earlier, the time to heat up the pool to $212^{\circ} \mathrm{F}$ is estimated to be 56.2 to 36.4 $\mathrm{hr}$ for this range of initial pool temperatures. At the maximum pool temperature allowed by Tech Specs $\left(170^{\circ} \mathrm{F}\right)$ the time to heat the pool to $212^{\circ} \mathrm{F}$ is 16.6 hr.

The pool boiloff rate can be estimated using the following expression.

$$
W_{B}=P / h_{f y}
$$

where

$$
\begin{aligned}
W_{B} & =\text { Pool boil off rate, } 1 b_{m} / h r \\
h_{f g} & =\text { heat of vaporization, Btu/1 } b_{m} \\
P & =\text { Pool heat load, Btu/hr }
\end{aligned}
$$

For the case considered here, $P=11.32 \times 10^{6} \mathrm{Btu} / \mathrm{hr}$ and $h_{f g}=970.3$

Btu $/ 1 b_{m}$ for $212^{\circ} \mathrm{F}$ saturation temperature

$$
W_{B}=11.32 \times 106 / 970=11,7001 \mathrm{~b} / \mathrm{hr} .
$$

Prior to the transfer of spent fuel from the reactor to the spent fuel storage pool, water will enter the cladding of those pins which have experienced failure in operation. If, during the heatup of the pool, boiling occurs in the gap of these failed pins, it is likely that a release of radioiodine will occur to the pool water analogous to the spike that occurs at the 
time of reactor startup after a short shutdown period. The mechanisnis of spiking are not adequately understood to accurately predict the size of the spike that would occur. General Electric(13), estimates that startup spikes are typically half as large as decompression spikes. This appears to be consistent with PWR behavior in which the inventory of jodine available for release is noticeably depleted by the occurrence of a spike such that subsequent spiking conditions within the period of 1 to 2 days lead to smaller spikes $(14)$.

The quantity of radioiodine release in a spiking sequence in an operating reactor can be estimated fran data tabulated by Pasedag(15), (see Appendix F) by fitting the data for total release as a function of iodine release rate.

$$
Q=e^{0.70} \pm 1.05 R I
$$

where $Q=$ total release of $\mathrm{I}_{131}$ in spiking sequence $(\mathrm{C} i)$

$\mathrm{RI}=$ equilibrium release rate of iodine $(\mu \mathrm{Ci} / \mathrm{sec})$ observed prior to spiking sequence

The variation in the exponent represents the distribution of observed spikes. The equilibrium concentration of iodine is related to the release rate as:

$$
\begin{aligned}
R I & =C I\left(\frac{F}{V}+\lambda_{d}\right) V \\
& =4900 \mathrm{CI}
\end{aligned}
$$

where

$$
\begin{aligned}
C I & =\text { equilibrium concentration }(\mu \mathrm{Ci} / \mathrm{g}) \\
F & =\text { purification flow rate }=4670 \mathrm{~g} / \mathrm{s} \\
V & =\text { primary system mass }=2.5 \times 10^{8} \mathrm{~g} \\
\lambda_{d} & =\text { decay rate }=9.96 \times 10^{-7} \mathrm{sec}^{-1}
\end{aligned}
$$


Relating iodine release rate to failed fuel fraction (assuming . $12 \%$ failed fuel is equivalent to a $.27 \mu \mathrm{Ci} / \mathrm{gram}$ of I-131 activity level in the primary coolant),

$$
Q=1.1 \times 10^{4} e^{0.70 \pm 1.05} \mathrm{FF}
$$

where $F F=$ percentage of failed fuel $(\%)$.

If the fraction of failed fuel is assumed proportional to burnup, for a burnup of 33,000 MWd/MT the failed fuel fraction would be $0.40 \% .67$ An upper bound of $1 \%$ and a lower bound of $0.12 \%$ can be assumed.

It is only necessary to consider the release of iodine from the last onethird section of the core to be placed in the SFSP. Reducing the iodine release by a factor of 2 to account for the smaller release in startup spikes and accounting for $I_{131}$ decay since shutdown, the spike release $Q$ is given by

$$
Q=5.5 \times 10^{3} e^{0.70 \pm 1.05} \times F C \times F F \times e^{-3.58 \times 10^{-3 \Delta t}}
$$

where

$$
\begin{aligned}
F C & =\text { fraction of core (usually } 1 / 3) \\
F F & =\text { percentage of failed fuel (\%) } \\
\Delta t & =\text { time from shutdown to start of boiling (hr). }
\end{aligned}
$$

It will be assumed that some fraction of the iodine released to the pool is in the form of volatile organic iodine. At the present time there is no good basis for estimating the fractional amount. Discussions with A. Postma indicated that he feels as high as 10 percent of the iodine can be converted to methyl iodide.

For organic iodine, the decontamination factor in the SFSP has been taken as unity. For inorganic iodine, as the pool boils the iodine will be partitioned between the liquid and the vapor. The partition coefficient, PC, for this process is defined as 


$$
P C=\frac{\text { Concentration of iodine in liquid }}{\text { Concentration of iodine in vapor }}
$$

Based on experinental data presented by Styrikovich, et al. (44), a minimum value of $10^{4}$ is expected for the partition coefficient. The corresponding decontamination factor, DF, is given by

$$
D F=\left(\rho_{\ell} / \rho_{g}\right) / P C
$$

where $\left(\rho_{\ell} / \rho_{g}\right)=$ the density ratio at the boiling pool conditions. At a saturation temperature of $212^{\circ} \mathrm{F}, \rho_{\ell} / \rho_{g}=1600$. This results in a decontamination factor of 0.16 .

In the event of an increase in radiation levels in the fuel building which contains the spent fuel pit, the fuel building ventilation exhaust can be diverted from the ventilation vent to roughing, particulate, and activated charcoal filters for radioactivity removal (Surry SAR p. 9.13.4-2). These filter banks are designed to remove 99.97 percent of solid particles down to 0.3 microns in size and 99.9 percent of any methyl iodide or iodine vapor entrained in the ventilation exhaust (Surry SAR p. 13.2-1).

In a boiling pool incident, diversion of the fuel building exhaust to the filter banks for radioactivity removal will place a high humidity load on the filter banks. The adsorption efficiency of a charcoal filter is a function of the temperature and humidity of the gas steam passing through the filter. Information available in ORNL-NSIC-65(45) and the Nuclear Air Cleaning 
Handbook (46) provides the following summary on the efficiencies of nuclear grade charcoal. The efficiencies are based on 1 -inch bed depth for elemental iodine efficiency and 2-inch bed depth for methyl iodide efficiency.

(1) Nonimpregnated charcoals: Efficiency for elemental iodine is satisfactory (over 99\%) even after extended operation in hightemperature $\left(260\right.$ to $280^{\circ} \mathrm{F}$ ) environuent containing steam and water droplets. Efficiency for methyl iodide is satisfactory at relative humidities less than $70 \%$ but nil at high (over $80 \%$ ) humidity.

(2) Impregnated charcoals: Efficiency for elemental iodine is satisfactory (over 99\%) under all temperature and humidity conditions, up to $270^{\circ} \mathrm{F}$ and $100 \%$ relative humidity. Single-pass efficiency with 0.2 sec residence time is given in Table J3. Flooding of the carbon due to free water may reduce efficiency to as 1 ow as $20 \%$.

The long term performance of charcoal filters in removing radioiodine in ventilation exhaust air in a reactor plant has been investigated in an EPRIsponsored study. (47) The study indicated that the charcoal filters showed no reduction in retention efficiency for elemental and inorganic forms of iodine over a three year period of continuous use. For organic iodine, the retention efficiency was found to decrease with time. After three years of use, the filter efficiency was approximately one-half of the efficiency observed after the first year of use.

Based on the above information, best estimates for filter efficiencies at the high humidity conditions considered here are 99.9 percent for el emental iodine with a range of 99 to 99.99 percent and 30 percent for organic iodine with a range of 10 to 90 percent. 
TABLE J3

RECOMMENDED DESIGN VALUES FOR SINGLE-PASS

METHYL IODIDE EFFICIENCY FULL-SCALE ADSORBERS

CONTAINING IMPREGNATED ACTIVATED CARBON

\begin{tabular}{|c|c|c|}
\hline \multirow{2}{*}{$\begin{array}{l}\text { Relative } \\
\text { Humidity } \\
(\%)\end{array}$} & \multicolumn{2}{|c|}{$\begin{array}{c}\text { Percent Efficiency for } \\
\text { Radioiodine as } \\
\text { Methyl Iodide }\end{array}$} \\
\hline & $70^{\circ} \mathrm{F}$ & $270^{\circ} \mathrm{F}$ \\
\hline 85 or less & 95 & 98 \\
\hline 90 & 90 & 90 \\
\hline 95 & 80 & 70 \\
\hline 98 & 70 & 30 \\
\hline
\end{tabular}

2-in. bed depth, $0.2 \mathrm{sec}$ residence time, $3 \mathrm{mg}$ radioiodine as methyl iodide per gram of carbon 


\section{J.2 Spent Fuel Pit Probabilistic Analysis}

The estimation of risk for the spent fuel pit loss of cooling accident sequence requires incorporating the factors defined in the previous subsection into an analysis methodology. The objective of this methodology is to estimate probability versus release magnitude for this sequence, and bound this estimate based on the uncertainty in the parameters upon which the estimate depends. All relevant factors that could affect the probability or release magnitude are to be included.

The results presented here are estimated from the distributions defined in the previous section. In some cases the upper bounds of distributions were used to obtain conservative results in a timely manner. However, for those factors that appeared to have the largest affect on the probability versus release magnitude curve, the entire distribution of possibilities was incorporated into the analysis. The partial failure analysis technique was chosen to incorporate the statistical aspects of the problem into an estimate of probability versus release magnitude.

The analysis methodology was structured to account for the large number of dependencies among the factors affecting the probability versus release magnitude estimate. Most of these factors are, however, dependent upon the time after refueling at which the accident (loss of pit cooling) occurs. For instance, the time after refueling $\left(t_{1}\right)$ at which the accident occurs affects:

- The radioactive inventory of the fuel rods

- The heat load to the pool, which in turn affects the time required to raise the fuel pit to boiling $\left(t_{2}\right)$, and the boiloff rate

- By virtue of $t_{2}$ above, the probability of the pit reaching boiling

- By virtue of the boil-off rate, the rate at which $1-131$ is released to the atmosphere. 
Therefore, the analysis methodology was structured around the dependencies inherent in the time after refueling at which the accident occurs. If it is assumed that the accident occurs on a given day after refueling, the relationships between the various factors affecting probability versus release magnitude can be defined and incorporated into the methodology. This suggests a partition of the sample space by day after last refueling, which was the approach taken. The probabilities versus release magnitude are estimated given that the accident occurs on each of the specified days after refueling, and the results are summed using the law of total probability to obtain the probability versus release magnitude for the accident sequence. Other aspects of the problem treated by the analysis include:

- Meshing accident sequences involving binomial (failed/not failed) failures and continuous random variables that affect the probability and release magnitude (e.g., time after refueling when the accident occurs; percent failed fuel; time required to repair failed pumps or valves).

- Separation of the uncertainty in the parameters affecting probability or release magnitude into random (variation) and systematic (ignorance) components. The parameters exhibiting random error, or variation, are used to define the shape and centrality of the probability versus release magnitude curve. The parameters exhibiting systematic error, or ignorance, are used to estimate the bounds on the probability versus release magnitude curve.

Concerning the second item above, parameters exhibiting random error, or variation, generally represent possible states of the system at the time of accident. If one were to sample a random reactor at a random time, one would find a state of the system defined by a certain time after refueling, a certain failed fuel percent, etc. On the other hand, parameters exhibiting systematic error, or ignorance, have only one value for all reactors (e.g., the true value of the partition factor), but we are ignorant of this true value, and have instead a distribution of possible values. 
Succeeding sections treat the model development, and results, in more detail.

A partial failure model was constructed to estimate the probability versus release manitude for the fuel pit loss of cooling accident. Figure $J 1$ shows a flow diagram illustrating the major events required to release radioactivity in this accident. Referring to Figure $\mathrm{J} 1$, the probability of loss of pit cooling was estimated from a fault tree analysis of the Surry spent fuel pit cooling system. The other factors in Figure $J_{1}$ were included in a partial failure analysis, since the release magnitude depends on a number of continuous random variables, e.g., failed fuel percent, activity level of the spike following boiling, duration of boiling, boiling rate, etc.

The model partitions the sample space by time after refueling at which cooling is lost. Define the events:

$A_{i}=$ The event that cooling is lost on the $i$ th day after refueling, and the accident results in pit boiling

$R_{j}=A$ specified release magnitude of size $R_{j}$

By the law of total probability, the probability of the specified release magnitude greater than $R_{j}$, from this accident sequence is:

$$
P\left[R_{j}\right]=\sum_{i=k}^{n} P\left[R_{j} / A_{i}\right] P\left[A_{i}\right]
$$

where: $n$ refers to the summation over the $n$ days between refuelings,

$$
\sum_{i=k} \begin{aligned}
& \text { starting with the } k^{\text {th }} \text { day ( } k=5 \text { for the analysis reported } \\
& \text { herin) }
\end{aligned}
$$

For the $i^{\text {th }}$ day after refueling, the model estimates the conditional probability of release greater than $R_{j}$ given that loss of cooling occurs on the $i$ th day $\left(P\left[R_{j} / A_{j}\right]\right)$, and the marginal probability that loss of cooling occurs on the $i$ th day $\left(P\left[A_{i}\right]\right)$. These two probabilities are estimated 


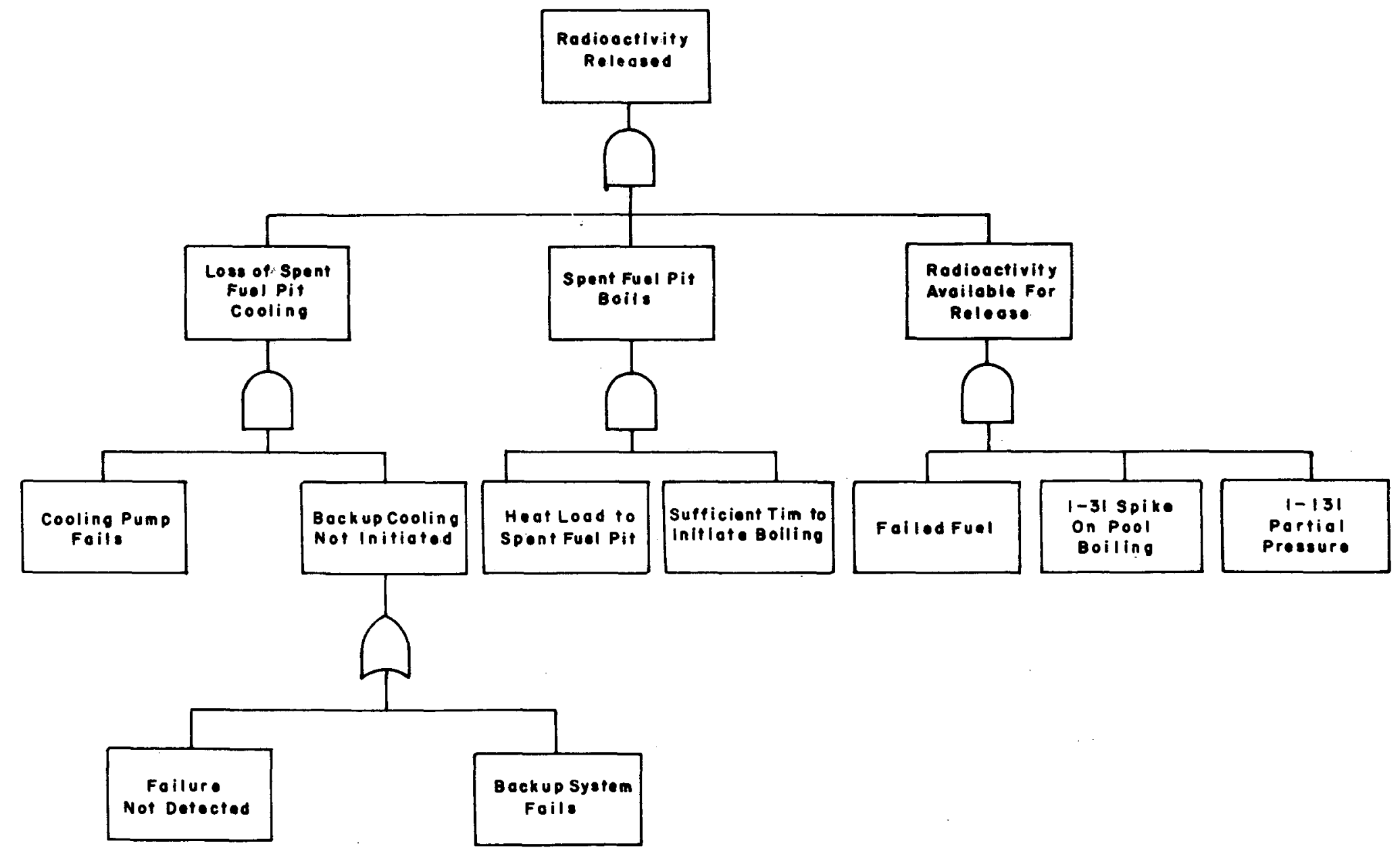

Figure J1. Schematic fault tree for radioacitivity released from the spent fuel pool 
by different techniques. The conditional probability is estimated by a partial failure analysis from a consideration of the factors that define the state of the system at the time of the accident, e.g., failed fuel percent, time required to repair the cooling system or mitigate the accident, etc. The marginal probability is estimated from a fault tree analysis of the fuel pit cooling system.

Both the marginal and conditional probability estimates are uncertain by virtue of the systematic error in the parameters upon which these estimates depend. The marginal probability estimate, $P\left[A_{i}\right]$, is uncertain due to the uncertainty in the failure rates of component failures leading to the event "the fuel pit begins to boil." The conditional probability, $P\left[R_{j} / A_{j}\right]$, is uncertain due to uncertainty in several physics and engineering parameters, namely the I-131 partition factor, and filter efficiencies in the hot, humid conditions that would result from a boiling spent fuel pit. Thus, both the marginal and conditional probabilities are more correctly thought of as distributions of possible probabilities for the accident sequence. These distributions reflect the best estimates of the systematic error in the parameters that are used to estimate the probabilities.

The probability of a specified release magnitude greater than $R_{j}$, $P\left[R_{j}\right]$, is thus also a distribution, since $P\left[R_{j}\right]$ is the sum or products of the conditional and marginal probabilities, both of which are distributions. To estimate the distribution of $P\left[R_{j}\right]$, the model propagates the distributions of $P\left[R_{j} / A_{j}\right]$, and $P\left[A_{j}\right]$ to $P\left[R_{j}\right]$ using equation (3-1). The model performs this error propagation for several specified values of $R_{j} \cdot$ The medians of the distributions for $P\left[R_{j}\right]$ define the centrality and shape of the probability versus release magnitude curve for the boiling fuel pit 
accident. The five and ninety-five percent confidence levels of the distributions for $P\left[R_{j}\right]$ serve to bound the probability versus release magnitude. The end product of the modeling effort is a complementary cumulative curve for probability versus release magnitude, and confidence bounds on the curve.

The detailed methodology employed by the model to estimate the distributions for $P\left[R_{j} / A_{j}\right]$ and $P\left[A_{j}\right]$ is presented next.

The distribution for the marginal probability, $P\left[A_{j}\right]$, is estimated using a fault tree analysis of the spent fuel pit cooling system. An analysis of the cooling system and associated piping indicated that the most likely accident sequences leading to a boiling spent fuel pit were those associated with a failure of the spent fuel pit cooling system and subsequent heatup of the spent fuel pit water to boiling. Section J.3 contains a description of this scoping analysis. Section J.3 also contains a schematic diagram of the Surry spent fuel pit cooling system, upon which the fault tree analysis was performed, and a detailed description of each fault considered. The fault tree analysis performed to assess the distribution of $P\left[A_{j}\right]$ is identical to the example analysis in Section J.3, with the following modification:

- The initiating event for the example analysis of Section J.3 for cooling system failure is cooling pump fails to run. The probability of the initiating event in Section J.3 was computed over a period of one year, so that the results appear as probability of spent fuel pit boiling per reactor year. The time span for the initiating event for the model results reported herein was taken as the time between refueling of two reactors which is assumed to be approximately one half year (150 days).

- The surveillance probabilities of the example analysis of Section J.3 were computed for one assumption concerning time after refueling at which the accident occurred (four days), and a conservative estimate of initial fuel pit water temperature of $170^{\circ} \mathrm{F}$. Thus, the time between initiating event and pit boiling is conservatively small, and the probability of surveillance failure is conservatively large. Realistic values were used to generate the model results reported herein. 
- Possible repair of equipment during the interval between the initiating event and the on-set of boiling was not considered in the example analysis of Section D.3. This was considered, however, for the model results reported herein.

Otherwise, the fault tree upon which the distribution of $P\left[A_{j}\right]$ was evaluated was the same as the fault tree shown in Section J.3.

Figure 32 shows a top level fault tree identifying the major classes of cooling system failures leading to spent fuel pit boiling. Figure 32 details specific pump and valve failures that would fail the backup system. The initiating event for the accident is failure of a cooling pump sometime within a span of time of interest (e.g., time between refuelings). The evaluation of the fault tree depends on the time between the initiating event and the onset of boiling $\left(t_{2}\right)$, since this affects both the probability of failure of human surveillance, and the probability of failure to repair the cooling pump or backup system. The time $t_{2}$ depends on the heat load to the spent fuel pool, which in turn depends on the time after refueling at which the cooling pump fails. Thus, it is necessary to multiply the probability of the initiating event occurring in a given time span (e.g., time between refueling) by the probability that the initiating event occurs on exactly the $i^{\text {th }}$ day after refueling. Therefore, the distribution of marginal probabilities obtained from a quantification of the fault tree is multiplied by $I / N$, where $N$ is the number of days between refuelings ( $N=150$ days for the results presented here). The probability of failure of human surveillance was estimated based on the following assumptions:

- If the accident occurred such that the accident and boiling were both initiated over a weekend, it was assumed that insufficient personnel would be available to detect the accident before boiling occurred; i.e., personnel are not in the fuel pit area over the weekend. 


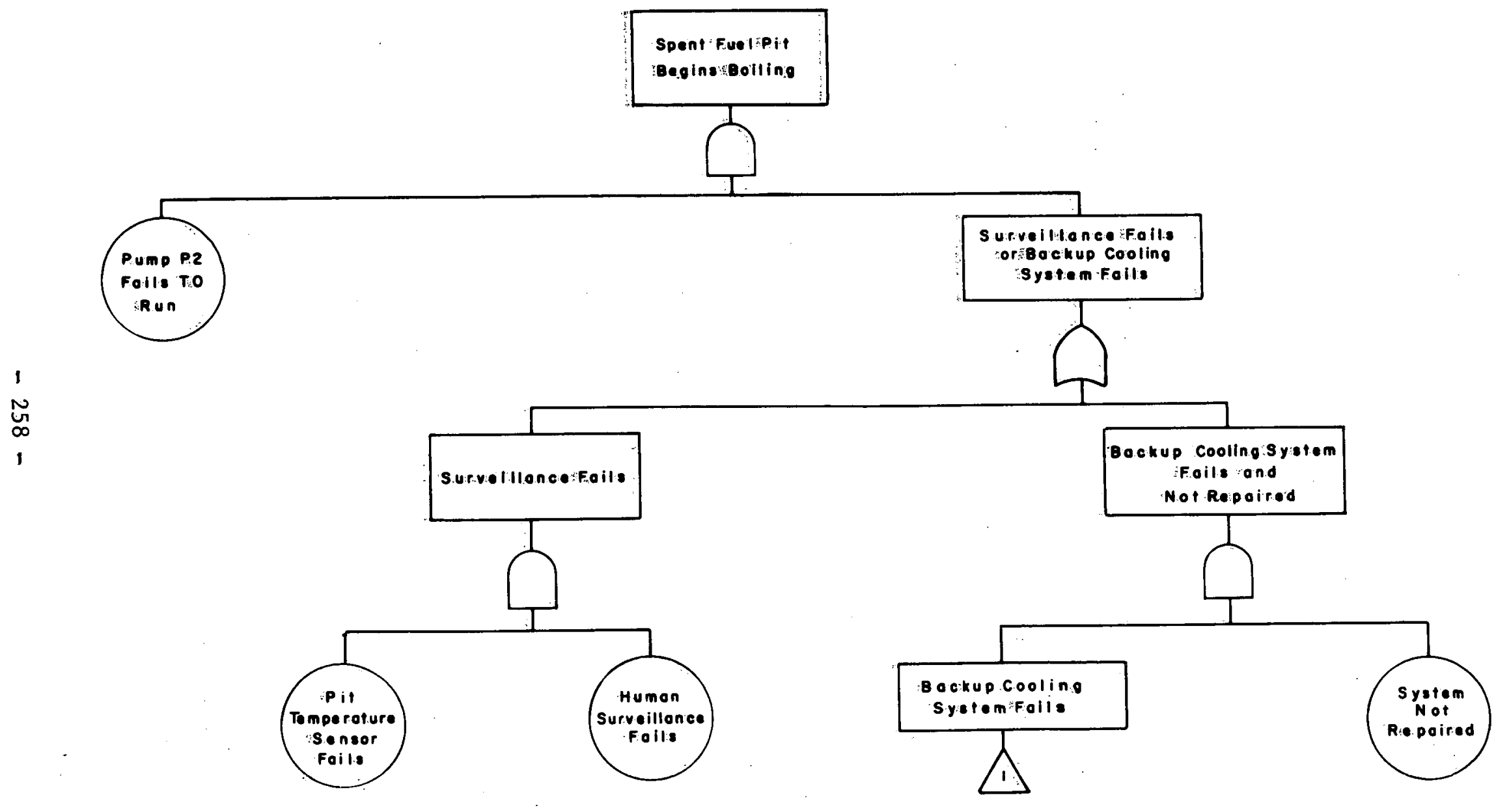

Figure J2. Schematic fault tree for boiling of spent fuel pool 


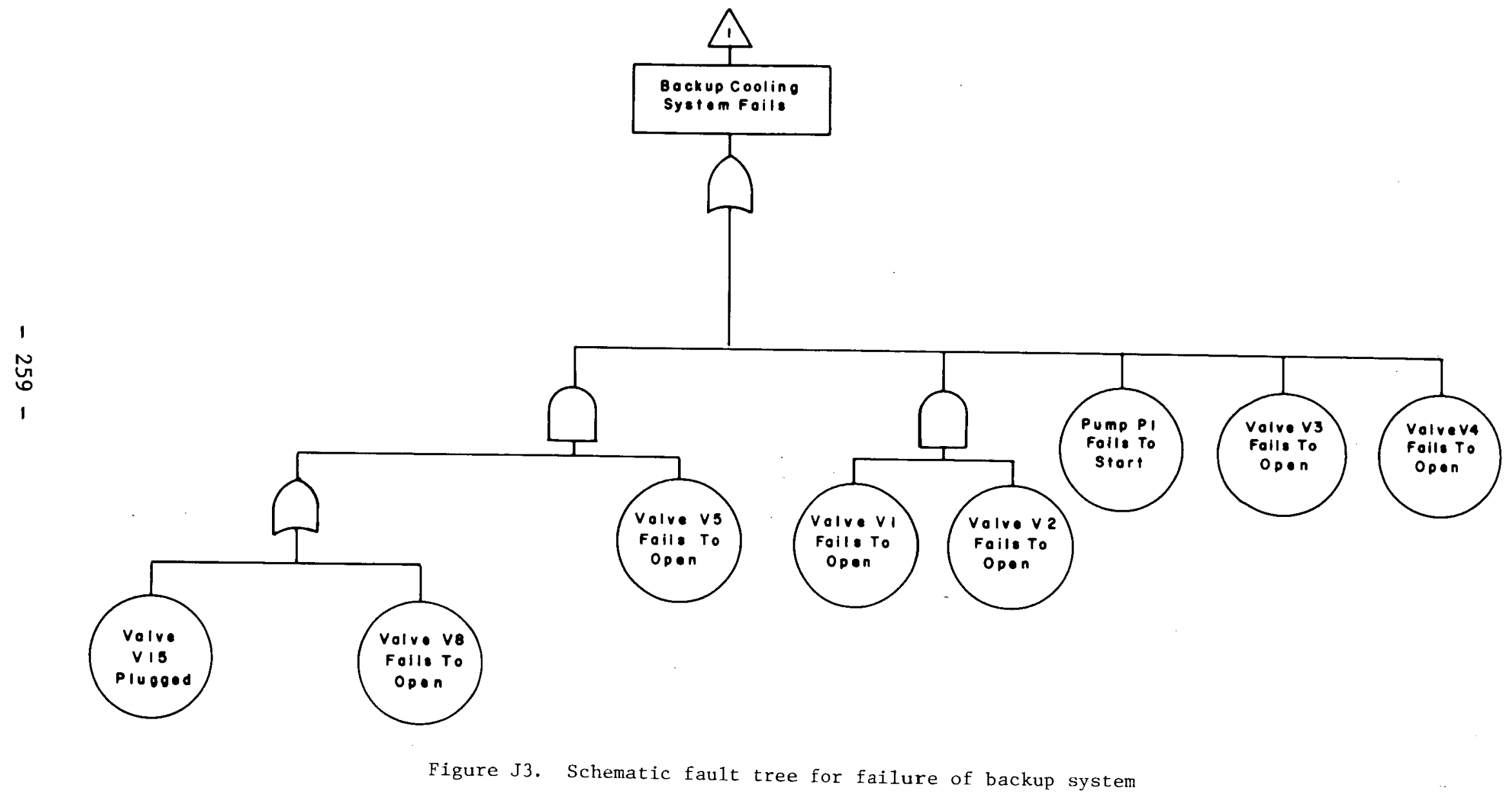


If the accident occurred such that the accident or boiling were initiated during the week, the accident would be detected by personnel in the fuel pit area.

Thus, a "window" is defined such that if the accident occurs in this window, it is not detected. The probability of no human surveillance in the window is computed as:

$$
P[S]=\frac{\left(63-t_{2}\right)}{168} \text { per demand }
$$

where $P[S]=$ probability of absense of human surveillance

$63=$ number of hours in the weekend

$168=$ number of hours in a full week

$t_{2}=$ time between initiating event and the on-set of boiling. The expression for $P[S]$ is a function of $t_{2}$, which in turn is a function of the time after refueling when the accident occurs.

The probability that the system is not repaired before the onset of boiling was estimated from the pump repair time distribution presented in WASH 1400, (1) Appendix III. This distribution is presented as Figure 34, for convenience. The probability is also a function of $t_{2}$, and was evaluated from a polynomial fit to Figure 34 .

The distribution of $P\left[A_{j}\right]$ was evaluated over a range of values for $t_{2}$ using the SAMPLE computer program. The range of $t_{2}$ was chosen to reasonably span the values of $t_{2}$ that would be required to evaluate probability versus release magnitude for the spent fuel pit loss of cooling accident. Figures 35 and $J 6$ show the median and error factor for the distribution of $P\left[A_{j}\right]$ over the range of $t_{2}$. These curves were piecewise fit to polynomial expressions and used in the model to evaluate the distribution for $P\left[A_{j}\right]$ for each required time after refueling, $t_{1}$. Thus, the distribution for $P\left[A_{j}\right]$ was retrieved when required by the model. 


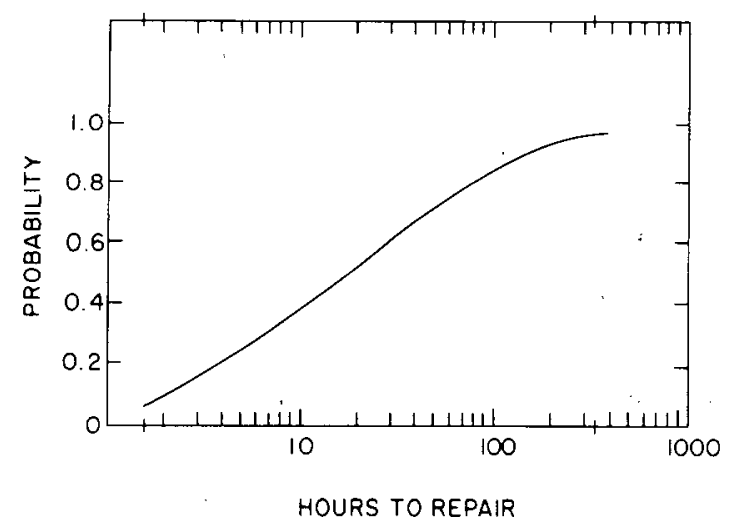

Figure J4. Probability distribution for repair time of pumps

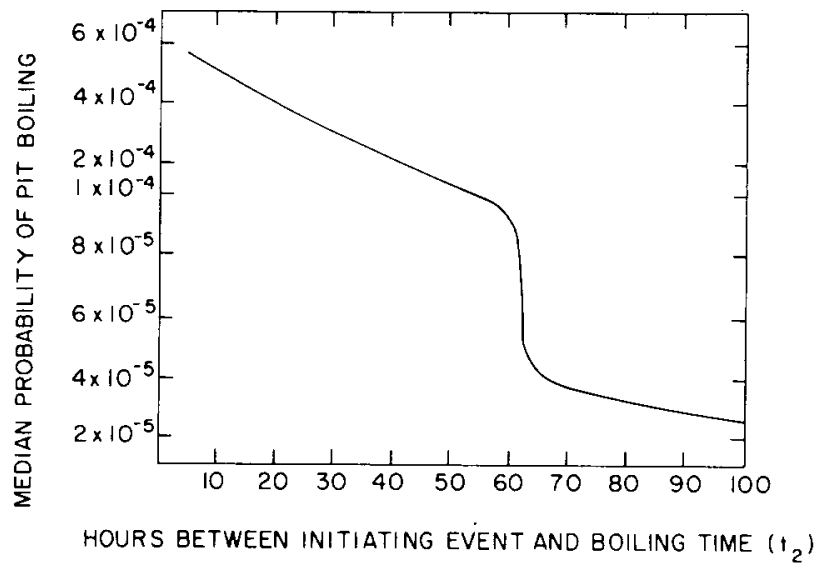

Figure J5. Median probability of pit boiling

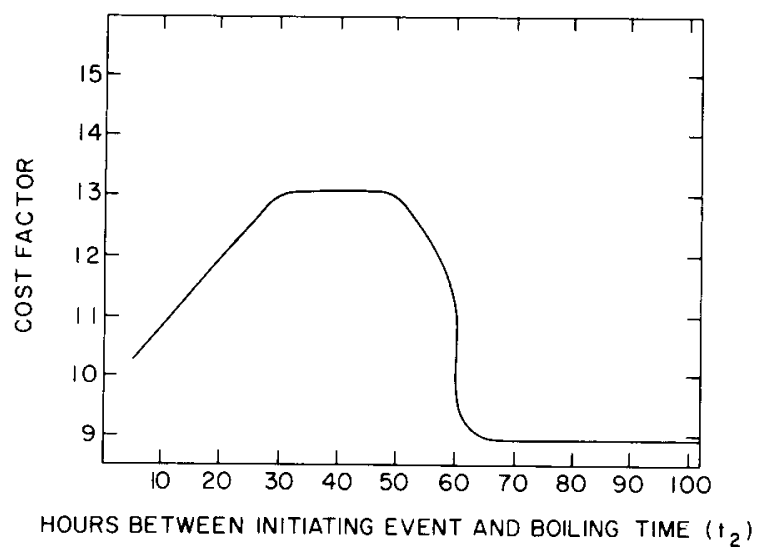

Figure J6. Error factor for probability of pit boiling 
By expressing the distribution of $P\left[A_{j}\right]$ in terms of a median and error factor, the implicit assumption is that this distribution is log-normal. This assumption is a reasonably good one for the cases at hand. The error factor for a given value of $t_{2}$ can be estimated as the ninety-fifth percentile of the distribution divided by the median, or as the median divided by the fifth percentile of the distribution. For a log-normal distribution, these calculations yield the same error factor. For the cases evaluated in this problem, the estimates did not differ by more than 30 percent. The error factor estimated by the ninety-fifth percentile divided by the median was used to approximate the upper bound of the probability versus release magnitude curve. 


\section{J.3 Spent Fuel Pit Fault Tree Analysis}

The description and data used are taken from the Surry FSAR. The fuel pit cooling system consists of two full size coolers and two full size circulating pumps that are cross-connected to provide 100 percent backup for both cooling and circulation requirements. One cooler and one pump are nomally used as backup systems and are cut out of the cooling loop by pneunatically operated valves. By opening nomally closed valves and closing nomally open valves, either of the two coolers or the two pumps may be interchanged, in the event of cooler or purnp failure, or due to maintenance requirements. The purification loop inlet is connected to the outlet line of one of the coolers. This loop feeds the fuel pit ion exchanger and the fuel pit filter, either of which may be removed from the 10op. Dual pumps are connected into the loop to provide 100 percent redundancy. The skimmer loop consists of two independent pumps and skimmer filters that also provides 100 percent redundancy except at the pit return line which has a common seyment of pipe before feeding back into the pit through multiple nozzles. Table J4 lists the fuel pit cooling system characteristics.

The location of pipes and fuel assesmblies within the spent fuel pit as well as the relationships between the spent fuel pit, the pump rooms, the stairwell and hoist areas, and the new fuel entrance door are dimensionally illustrated in Figure 37 . The spent fuel pit is constructed of reinforced concrete 3 to 6 feet deep within 1/2 inch butt-welded stainless steel (304 SS) plate liners and designed to withstand Class I Design Basis Earthquake specifications. The top of the pit is open to permit access and the water is purified and skimmed to allow clear inspection of the pit and its contents. The normal water level in the pit is approximately $167 \mathrm{in}$. from the fuel top 
TABLE J4

SPENT FUEL PIT COOLING SYSTEM CHARACTERISTICS

FUEL PIT

Construction

Design

Capacity

Structure

Liner

Depth

COOLING LOOP
- Boron Water-filled open top pit (2500 ppm Boron)

- Seismic category class

- 570,000 gallons $(407,800$ gallons water with full spent fuel inventory

- 3 to 6 feet thick reinforced concrete

- 1/4" butt welded 304 stainless steel plate

- 462 inches

- 200\% (Two pumps and two coolers independent and cross connected)

Maximum Operating Temp. - $\leq 170^{\circ} \mathrm{F}$

Heat Transfer

Flow

Head

Min. Pipe Size

Max. Drain Size

PURIFICATION LOOP

Flow

Head

Min. Pipe Size

Max. Drain Size

SK IMMER LOOP

Flow

- $10 \mathrm{gpm}$

Head

- 30 feet

Min. Pipe Size

$-3 / 4$ inch

Max. Drain Size

$-3 / 4$ inch
- $34.75 \times 10^{6} \mathrm{Btu} / \mathrm{hr}$ (per cooler)

- $4200 \mathrm{gpm}$

- 62 feet

- 12 inches

- 1 inch (2-1/2" through Purification Loop)

- $150 \mathrm{gpr}$

- 198 feet

- 2-1/2 inches

- 2-1/2 inches (per pump)

Ref: FSAR Table 9.5-1 


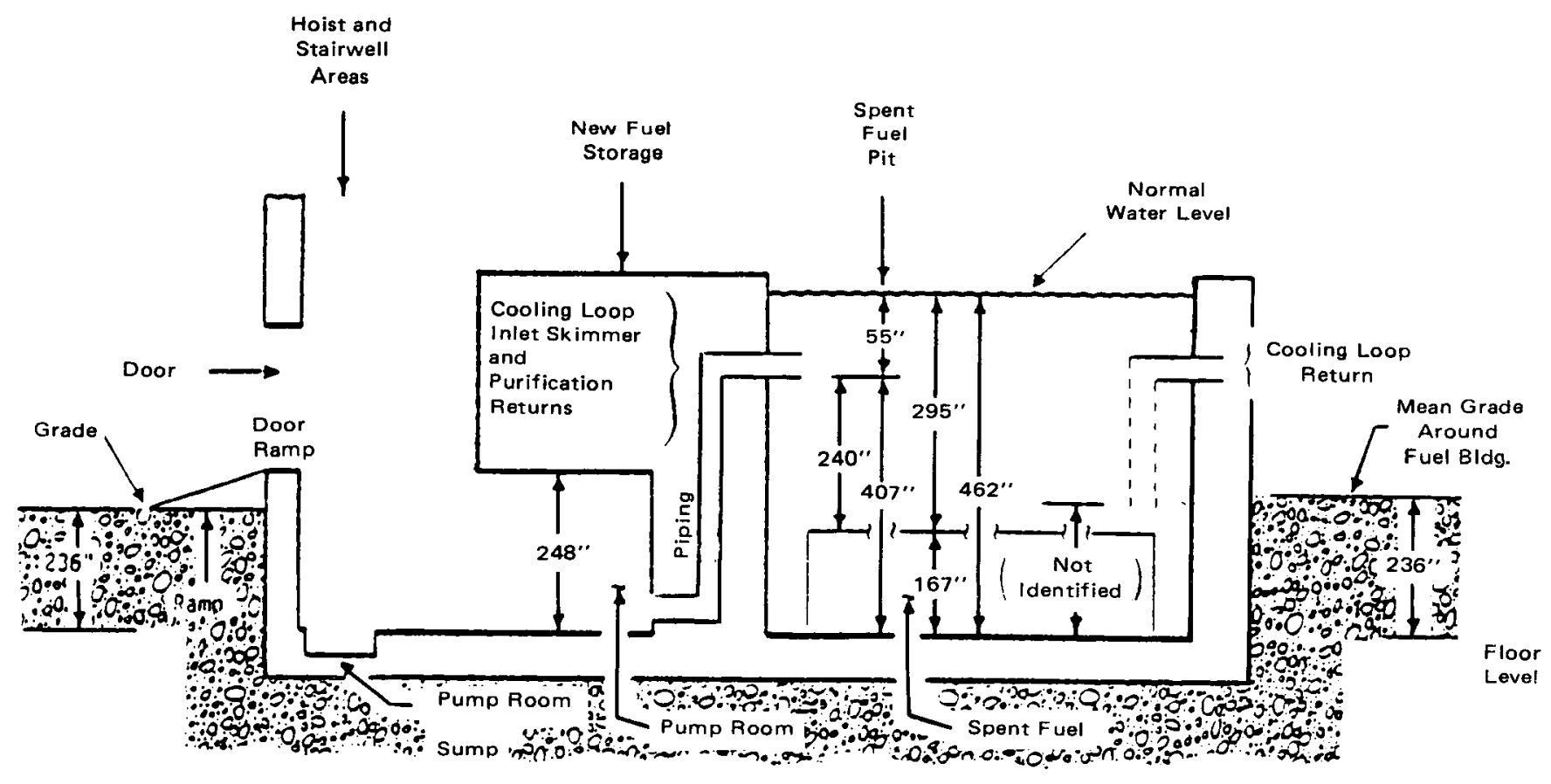

Figure J7. Dimensiona1 schematic of Surry spent fuel pit 
295 in. from the pit floor. The Surry FSAR states that the lowest level of pipe penetrations through the fuel pit structure is 20 feet or $240 \mathrm{in}$. above the top of the stored fuel elements.

The projection of the cooling, purification, and the skimming pipes below this level is not specified; however, the $16 \mathrm{in}$. cooling return line is shown on the fuel pit systeri functional diagram to extend downward after penetrating the structure. The pumps, coolers, and filters are located in the pump room on a level with the bottom of the pit. The ceiling of this room is approximately 248 in. above the floor with a stairwell and hoist areas located in one end of the fuel building with the pit taking up the other end of the building.

The spent fuel pit water level and temperature are both monitored on event recorders in the power plant control room. The water level event recorder flags both high and low water levels and the temperature recorder flags a "Hi Temp" as well as a "Hi Hi Temp" spent fuel pit water temperature. An alarm is also provided on the pool to sound at a level loss of approximately $1 / 2 \mathrm{ft}$. slow leakage of water from any power in the piping or components or the cooling or purification systems can be stopped by valves mounted close to the pool penetrations. Further, the FSAR states "large piping systern leak can reduce the water level in the pool to only $4 \mathrm{ft}$ below nomal since at this elevation the water level is below pipe penetration in the pool wall."* However, the cooling loop return is shown on the system functional diagrams as penetrating the pool below this level. Although this return pipe no doubt has antisiphon holes, should these holes become plugged for some reason it is possible that the pit water could be siphoned down to the level of the bottom of this pipe.

*Neither the FSAR drawings or specifications indicate the presence of ventilation directed across the surface of the pool. 
The most likely cause of loss of spent fuel pit water is vaporization due to loss of cooling. Loss of cooling may result from either leakdown of the water to below the cooling system inlet or due to malfunction within the cooling system with the subsequent vaporization of pit water. Although siphoning will not be ruled out in this analysis, siphoning below the pipe inlet appears unlikely based on current information derived from the FSAR.

Water escaping from the spent fuel pit, other than by vaporization, is expected to drain into the pump room; unt il the water level rises to the new fuel service door ramp level. How much water will drain out of the pit depends on the nature of the leak and/or the depth that the cooling loop return pipe extends into the pit water if and when the pit is being siphoned. Additional water escapiny from the pit can then drain through the doorway when the door is open or water escaping from the pit can build up in the stairway and hoist areas until the water levels in the pit and these areas stabilize.

The pump room volume below the new fuel service door ramp level is estimated to be approximately 194,500 gallons. The spent fuel pit is estimated to contain approximately 263,800 gallons above the level of the new fuel service door ramp. Therefore approximately 69,300 gallons of spent fuel pit water could flow out of the fuel building through this door. Should the new fuel service door be closed, the water could possibly build up in the hoist and stairwell area at a rate of approximately 294 gallons per inch of rise in water level which would result in a stabilized water level between the water in the spent fuel pit and lost water of approximately $45 \mathrm{in}$. above the new fuel service door ramp level. The amount of water released to the environment with the new fuel service door first open and then closed is. sunmarized in Table $\mathrm{J} 5$ along with the radiation shield remaining after the leakdown stabilizes. 
The elapsed time between the occurrence of a fault event that will result in the loss of spent fuel pit water and the exposure of the top of the spent fuel assemblies has been calculated for five combinations of leakdown, siphoning and vaporization. The detailed schedule calculations are presented in Table $\mathrm{J} 6$ with the results of these calculations sumarized in Table $\mathrm{J} 7$.

The calculations for vaporization assume an initial fuel pit temperature of $170^{\circ} \mathrm{F}$ and a heat loading equal to the heat removal capacity of the fuel pit cooling system. Thus, these calculations are conservative and probabilities estimated from these numbers will tend to be larger than realism would suggest. However, the numbers are sufficient for comparison purposes, to indicate the fault sequences that dominate the analysis. This is exactly what they have been used for, in an example analysis.

The data in Table $J 7$ illustrates that although there is an order of maynitude difference in leakdown elapsed time between a $3 / 4$ in. and a 2-1/2 in. leak this difference contributes only approximately 20 percent to the total difference in elapsed time. The difference in the time required to heat up the spent fuel pit water from $170^{\circ} \mathrm{F}$ to $212^{\circ} \mathrm{F}$, as well as the differences in the vaporization times, reflects the difference in the amount of water that must be heated for each fault subevent case.

The primary means of losing spent fuel pit water have been identified as cooling system leakage, cooling system siphoning and vaporization due to loss of cooling. Other means of losing spent fuel pit water that were considered and determined not to be significant factors are the fuel building drain, the fuel transfer canal and natural and operational disasters.

The fuel building drain sump is located in the pump room floor. Drains from the fuel pit coolers overflow from the spent resin dewatering tank, and 
TABLE J5

\section{WATER RELEASED BY LEAKDOWN}

Remaining

Radiation Shield

\begin{tabular}{lrrr}
\hline $\begin{array}{c}\text { New Fuel } \\
\text { Service Door }\end{array}$ & in. & gal. & $\begin{array}{c}\text { Water Released } \\
\text { to environment (gal.) }\end{array}$ \\
Open & 80 & 99,500 & 69,300 \\
Closed & 125 & 154,400 & $-0-$
\end{tabular}


TABLE 36

ELAPSED TIME SCHEDULES FOR THE LOSS OF SPENT FUEL PIT COOLING WATER DUE TO LEAKDOWN, SIPHON AND VAPORIZATION

\begin{tabular}{|c|c|c|c|c|c|c|c|c|c|c|c|c|c|c|c|c|}
\hline \multirow{3}{*}{$\begin{array}{l}\text { Primary } \\
\text { Events }\end{array}$} & \multicolumn{2}{|c|}{ Height - (ft) } & \multirow{3}{*}{ 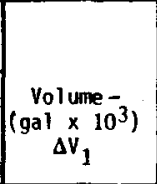 } & \multirow{3}{*}{$\mid \begin{array}{c}\text { Evapora- } \\
\text { tion Iime- } \\
\text { (hrs) } \\
t_{v}\end{array}$} & \multirow{3}{*}{$\begin{array}{c}\text { Water } \\
\text { Level } \\
\text { in Pump } \\
\text { Room- } \\
(\mathrm{ft}) \\
\mathrm{H}_{2} \\
\end{array}$} & \multirow{3}{*}{$\begin{array}{c}\text { Head- } \\
(\mathrm{ft}) \\
\Delta H\end{array}$} & \multicolumn{5}{|c|}{ 2h Inch Leak/Siphon Equivalent } & \multicolumn{5}{|c|}{ 3/4 Inch Leak/Siphon Equivalent } \\
\hline & De & Mean & & & & & \multirow[b]{2}{*}{$\begin{array}{l}\text { Flow } \\
\text { (gps) }\end{array}$} & \multirow{2}{*}{$\begin{array}{c}\text { Elapsed } \\
\text { Time - } \\
\text { (hrs) } \\
\text { TLS }\end{array}$} & \multirow{2}{*}{$\begin{array}{l}\text { Temp } \\
\left({ }^{\circ} \mathrm{F}\right)\end{array}$} & \multicolumn{2}{|c|}{ Elapsed Time (hrs) } & \multirow{2}{*}{ Flow } & \multirow{2}{*}{\begin{tabular}{|c|} 
Elapsed \\
Time- \\
(trs) \\
TLS \\
LS
\end{tabular}} & \multirow[b]{2}{*}{$\mid \begin{array}{l}\text { Temp } \\
\left({ }^{\circ} \mathrm{F}\right)\end{array}$} & \multicolumn{2}{|c|}{ Elapsed Time (hrs) } \\
\hline & $\mathrm{H}_{\mathrm{O}}$ & $\begin{array}{c}\text { Hedo- } \\
\mathrm{H}_{1}\end{array}$ & & & & & & & & $T_{\text {LSV }}$ & $T_{L V}$ & & & & $T_{\text {LSV }}$ & $T_{L V}$ \\
\hline (1) & (2) & (3) & (4) & (5) & (6) & (7) & (8) & (9) & (10) & (11) & (12) & (13) & (14) & (15) & (16) & (17) \\
\hline Normal Level & 38.50 & 36.22 & 67.4 & 15.6 & 3.59 & 32.64 & 10.52 & 1.8 & 170 & 1.8 & 1.8 & 0.96 & 19.5 & 170 & 19.5 & 19.5 \\
\hline \multirow[t]{4}{*}{ Pump Inlet } & & 32.87 & 31.8 & 7.4 & 8.86 & 24.01 & 9.02 & 1.0 & & 1.0 & $\begin{array}{l}3.4^{\star \star \star \star} \\
7.4^{-1}\end{array}$ & 0.82 & 10.8 & $\begin{array}{c}170 \\
(212)\end{array}$ & 6.4 & $\begin{array}{l}3.4^{\star \star *} \\
7.4\end{array}$ \\
\hline & 29.54 & 30.72 & 31.8 & 7.4 & 12.24 & 18.48 & 7.92 & 1.1 & & 1.1 & 7.4 & 0.72 & 12.3 & & 4.6 & 7.4 \\
\hline & 29.04 & 28.58 & 31.8 & 7.4 & $15.6 ?$ & 12.96 & 6.63 & 1.3 & $(212)^{\star \star}$ & 1.3 & 7.4 & 0.60 & 14.7 & & 4.9 & 7.4 \\
\hline & 0.0 & 26.43 & 31.8 & 7.4 & 19.00 & 7.43 & 5.02 & 1.8 & 1 & 1.4 & 7.4 & 0.46 & 19.2 & & 5.3 & 7.4 \\
\hline \multirow[t]{2}{*}{ Pump $R_{m}$ Filled } & 25.34 & 24.09 & 34.6 & 8.0 & 20.67 & 3.42 & 3.41 & $2.8^{\circ}$ & & 2.1 & 8.0 & 0.31 & 31.0 & & 6.4 & 8.0 \\
\hline & 23.01 & 21.75 & 34.6 & 8.0 & 20.67 & 1.08 & 1.91 & 5.0 & & 3.1 & 8.0 & 0.17 & 56.5 & & 7.0 & 8.0 \\
\hline Door Ramp* & 20.67 & 15.03 & 99.5 & 23.0 & 20.67 & $-0-$ & $-0-$ & $\infty$ & r & $23.0^{\star \star \star \star}$ & $23.0^{\star \star \star \star}$ & $-0-$ & $\infty$ & I & $23.0^{\star \star \star \star}$ & $23.0 * \star \star \star$ \\
\hline Top of Fue 1 & 13.94 & & & & & & & & & & & & & & & \\
\hline \multicolumn{4}{|c|}{ Time to Expose Fuel Assemblies w/o Back flow } & 84.2 & & & & & & 34.8 & 73.8 & & & & 77.1 & 91.5 \\
\hline \multicolumn{4}{|c|}{ Time to Expose Fue Assemblies with Back Flow } & 84.2 & & & & & & 49.4 & 88.4 & & & & 91.7 & 106.1 \\
\hline
\end{tabular}

- Total Pit Water Volume $\approx 407,800$ gallons. Water Volume above Fuel $\approx 363,300$ gallons.

** $212^{0} \mathrm{~F}$ Total Elapsed Iime $\approx 4.9 \mathrm{hrs}$.

$\star \star \star$ Time to heat up to boiling.

**** Assumes no back flow from pump room into spent fuel pit. With back flow both these vaporization elapsed times and the

total cummulative elapsed times would be increased by approximately $14.6 \mathrm{hrs}$. 
TABLE 37

SUMMARY TABLE OF THE TIME TO LOSE COOLING WATER FROM THE SPENT FUEL PIT

$\stackrel{N}{\forall}$

\begin{tabular}{|c|c|c|c|c|c|c|c|}
\hline \multirow{3}{*}{$\begin{array}{l}\text { Loss } \\
\text { Mechani isms }\end{array}$} & \multicolumn{6}{|c|}{$\begin{array}{l}\text { Water Escaping From Pit } \\
\text { (Drain Valve Left Open During Maintenance) }\end{array}$} & \multirow{3}{*}{$\begin{array}{l}\text { Loss of Cooling } \\
\text { (Pump Failure, Pump } \\
\text { or Cooler Transfer } \\
\text { Valve Failure) }\end{array}$} \\
\hline & \multicolumn{4}{|c|}{ Wi thout Siphon } & \multicolumn{2}{|c|}{ With Siphon* } & \\
\hline & $\sqrt{3 / 4^{\prime \prime} \text { Leak }}$ & 1" Leak & $2^{\prime \prime}$ Leak & $2 \frac{1}{2} "$ Leak & 3/4" Leak & $2 \frac{1}{2}$ " Leak & \\
\hline $\begin{array}{l}\text { 1: Leakdown Until } \\
\text { Below Cooling } \\
\text { Inlet }-t_{L}\end{array}$ & 19.5 & 11.0 & 2.7 & 1.8 & 19.5 & 1.8 & 0.0 \\
\hline $\begin{array}{l}\text { 2. Heatup }\left(170^{\circ} \text { to }\right. \\
212^{\circ} \mathrm{F}-t_{H}\end{array}$ & 3.4 & 3.4 & 3.4 & 3.4 & 3.4 & 3.1 & 4.1 \\
\hline $\begin{array}{l}\text { 3. Vaporize Water } \\
\text { Level Down to } \\
\text { Spent Fuel - } t_{V}\end{array}$ & 83.2 & 83.2 & 83.2 & 83.2 & 68.8 & 44.5 & 84.2 \\
\hline $\begin{array}{l}\text { TOTAL ELAPSED } \\
\text { TIME }-T() \text { (HRS) }\end{array}$ & 106.1 & 97.6 & 89.3 & 88.4 & 91.7 & 49.4 & 88.3 \\
\hline RELATIVE RANK** & 1.20 & 1.11 & 1.01 & 1.00 & 1.04 & 0.56 & 1.00 \\
\hline
\end{tabular}

* This column shows the maximum effect of siphon when siphon is assumed to start when water level drops below cooling inlet, and to continue until fuel is exposed.

** Using Loss of Cooling as Base Case. 
the fuel building floor and pit drains feed into this sump. Two pumps with a rated capacity of $25 \mathrm{gpm}$ at $74 \mathrm{ft}$ head are located in this sump to discharge the drainage through either the high level liquid waste drain filter into the high level liquid waste drain tank or through the low level liquid waste drain filter into the low level liquid waste drain filter into the low level liquid waste drain tank. The flow capacity of this drainage system is not large enough to significantly change the resultant water level or the rate of discharge when the pit is drained by a large leak into the pump room.

Spent fuel pit water also could escape from the fuel building through the fuel transfer canal into one of the reactor containment buildings. However, this fault condition is highly unlikely because the gate valves that close the transfer tubes are manualiy operated and only used during fuel transfer when the system is visually monitored by multiple operators. The additional factors that also tend to reduce this probability are first, the gate valve is backed up by a gate on the pit end of the transfer tube, and second, the capability of the containment building to accept leaked water is limited by the capacity of the building sump, the refueling water storage tank, and the waste water storage tank which is not large enough to significantly change the resultant water level or the rate of discharge when the pit is drained by a large leak into containment.

The plant is designed to comply with earthquake seismic category I engineering standards, therefore, an earthquake or other natural or operational disaster of considerable magnitude would be required to apply the force necessary to crack the pit wall and liner to allow spent fuel pit water to be released other than through the cooling system. The earthquake would also be required to open an earth fissure or provide other means to permit run-off of 
the escaping water before the pit area could be drained below the door ramp level which is still approximately 6-3/4 ft above the top of the stored spent fuel assembly. Risk assessment of earthquake induced faults affect the overall plant and, therefore, are beyond the scope of this analysis.

A fault tree analysis of the spent fuel pit was performed to identify the dominant accident sequences, and as a basis for assessing the probability that an accident sequence would result in the pit beginning to boil. This fault tree analysis is regarded as a scoping analys is since:

Only human errors and pump and value faults were considered in the analysis. The reliability of the pit coolers was not included on the fault tree, as this would have necessitated a fault tree analys is of these components.

- Human faults associated with failure of visual surveillance were estimated by assuming that no visual surveillance occurs over the weekend, and that the visual surveillance during the week is perfect. A better estimation of this fault would require the stockastic nature of visual inspection of the spent fuel pit, which was not readily available for the scoping analysis.

- The spent fuel pit level and temperature sensor failure probabilities were estimated on the assumption that these components are calibrated on a regular basis, such that common mode failure of both sensor systems may be possible. The human common model components of failure were used as the probability of failure of both sensors. Several valves are shown off $3 / 4$ " lines in the normally open position in the FSAR one line diagram. No indication is given as to the function of these valves, or where they connect to other systerns. These valves and their connecting systerns were not evaluated. 
Further, the possibility of siphon enhancing the leakdown rates was not assessed, since the probability of siphon should be small in a system that is designed with anti-siphon features.

Figure 38 shows a simplified one-line diagram of the spent fuel pit piping. The pit cooling systems, ion exchanger system, and filter system are shown in the top diagram; the skirnner system is shown in the bottom diagram. The fault tree analysis was performed on the top diagram only, since no credible failure modes were identified involving the skinmer system that would result in pit boiling.

Figure $\mathrm{J9}$ shows the fault tree developed fron Figure J8. Figure J9a is the top level tree, indicating the major failure modes that result in pit boiling. Five major fajlure modes are identified, along with pit sensor failures. The five major failure modes correspond to five different types of initiating events for pit boiling:

- direct loss of pit cooling

- leakdown of pit water to below the cooling system inlet, and subsequent loss of cooling.

The probability of the top event will depend on the times between the initiating event failures and subsequent pit boiling, since these times are used to estimate both the probability of failure of visual surveillance of the pit, and the probability that component repair is not completed in time to mitigate the accident. The times between the initiating events involving siphon and subsequent pit boiling were presented in Table $\mathrm{J7}$. These times are a function of the heat loading of the pit, which in turn is a function of the time after refueling, $t_{1}$. Thus, the probability of pit boiling will vary according to the time after refueling. 


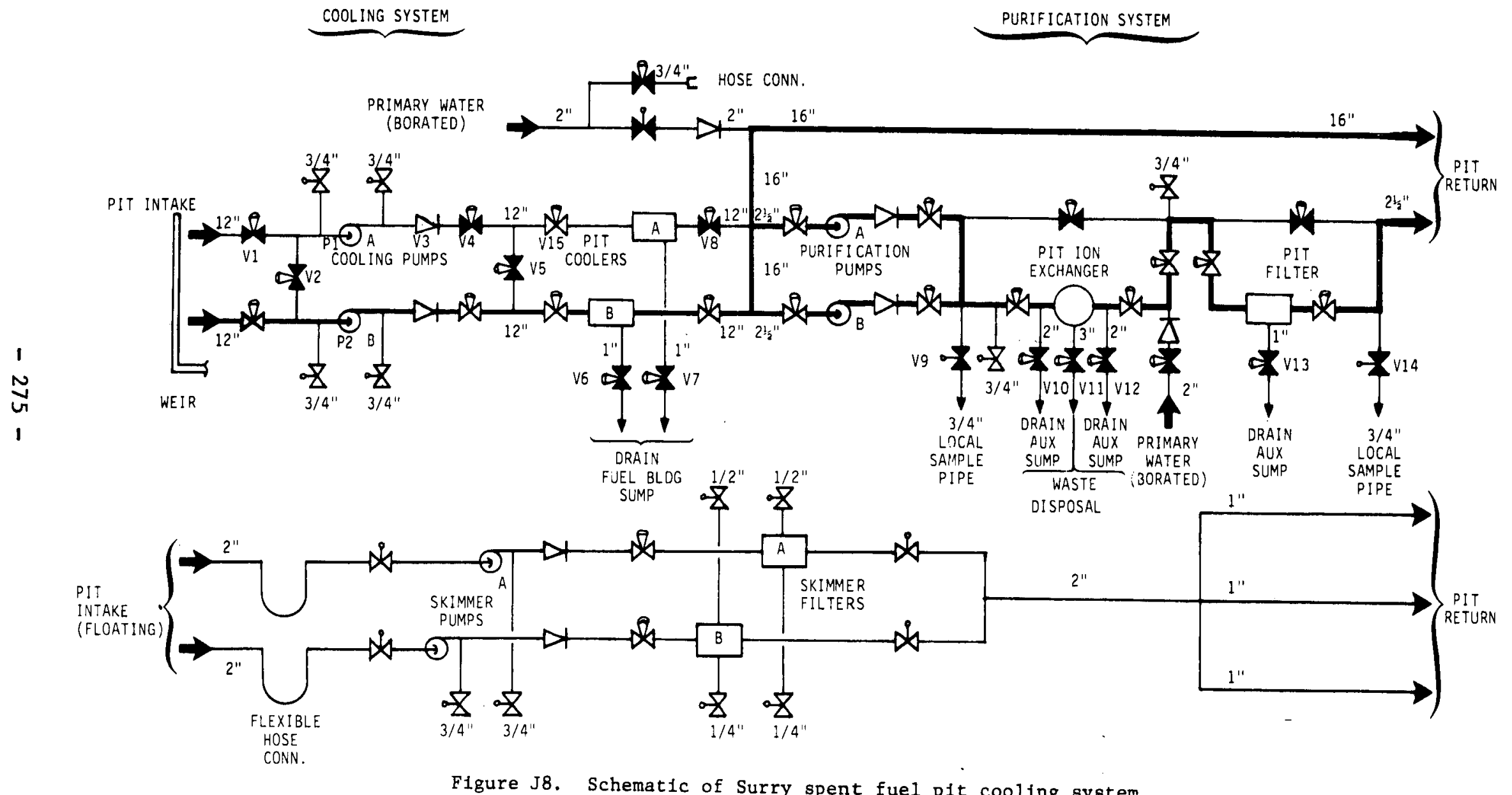


FAILURE DATA - SPENT FUEL PIT FAULT TREE EVALUATION

\begin{tabular}{|c|c|c|c|c|c|c|}
\hline System/Component & Failure Mode & Fallure Rate & $\begin{array}{l}\text { Fault } \\
\text { Duration } \\
\text { Time } \\
\end{array}$ & $\begin{array}{l}\text { Probability } \\
\text { of Failure }\end{array}$ & $\begin{array}{l}\text { Error } \\
\text { Factor }\end{array}$ & Remarks \\
\hline Temperature Sensor & Falls to Operate & $1 \times 10^{-2} / \mathrm{d}$ & & $1 \times 10^{-2}$ & 3 & $\begin{array}{l}\text { Based on miscalibration } \\
\text { of sensor }\end{array}$ \\
\hline Human Surveillance & Falls & $8.2 \times 10^{-2} / \mathrm{d}$ & & $8.2 \times 10$ & 10 & $\begin{array}{l}\text { Computed from time window } \\
\text { when there is no pit } \\
\text { survefllance } y\end{array}$ \\
\hline Pump P2 & Fails to Run & $3 \times 10^{-5} / \mathrm{hr}$ & $8640 \mathrm{hrs}$ & $2.6 \times 10^{-1} / y r$ & 10 & Inittating event \\
\hline Pneumatic Valve V4 & Fails to Open & $3 \times 10^{-4} / d$ & & $3 \times 10^{-4}$ & 3 & \\
\hline Check Valve V 3 & Fails to Open & $1 \times 10^{-4} / \mathrm{d}$ & & $1 \times 10^{-4}$ & 3 & \\
\hline Pump P & Fails to Start & $1 \times 10^{-3} / \mathrm{d}$ & & $1 \times 10^{-3}$ & 3 & \\
\hline Pneumatic Valve V2 & Fails to Open & $3 \times 10^{-4} / \mathrm{d}$ & & $3 \times 10^{-4}$ & 3 & \\
\hline Pneumatic Valve VI & Falls to open & $3 \times 10^{-4} / d$ & & $3 \times 10^{-4}$ & 3 & \\
\hline Pneumatic valve v5 & Fails to Open & $3 \times 10^{-4} / \mathrm{d}$ & & $3 \times 10^{-4}$ & 3 & \\
\hline Pneumatic valve v8 & Fatls to open & $3 \times 10^{-4} / \mathrm{d}$ & & $3 \times 10^{-4}$ & 3 & \\
\hline Pneumatic Valve V15 & Plugged & $1 \times 10^{-4} / d$ & & $1 \times 10^{-4}$ & 3 & \\
\hline $\begin{array}{l}\text { Level and Temp- } \\
\text { erature Sensors }\end{array}$ & Fail & $3 \times 10^{-4} / d$ & & $3 \times 10^{-4}$ & 3 & $\begin{array}{l}\text { Assessed as lognormal } \\
\text { mean between tightly } \\
\text { coupled, and independent, } \\
\text { calibration errors }\end{array}$ \\
\hline Valve VIl & Left Open (Human) & $1 \times 10^{-2} / \mathrm{act}$ & $2.7 \mathrm{acts} / \mathrm{yr}$ & $2.7 \times 10^{-2}$ & 3 & Initlating event \\
\hline $\begin{array}{l}\text { Human Surveillance } \\
(\mathrm{H} 2)\end{array}$ & Fatls & $8 \times 10^{-2} / d$ & & $8 \times 10^{-2}$ & 10 & $\begin{array}{l}\text { Computed from time window } \\
\text { when there is no pit } \\
\text { surveillance 2/ }\end{array}$ \\
\hline Vaive $V 12$ & Left Open (Human) & $1 \times 10^{-2} / a c t$ & 2.7 acts/yr & $2.7 \times 10^{-2}$ & 3 & Inttiating event \\
\hline Valve vio & Left Open (Human) & $1 \times 10^{-2} /$ act & $2.7 \mathrm{acts} / \mathrm{yr}$ & $2.7 \times 10^{-2}$ & 3 & Initiating event \\
\hline $\begin{array}{l}\text { Human Surveillance } \\
\text { (H3) }\end{array}$ & Fails & $7.9 \times 10^{-2} / d$ & & $7.9 \times 10^{-2}$ & 10 & $\begin{array}{l}\text { Computed from time window } \\
\text { when there is no pit } \\
\text { survetilance } 3 /\end{array}$ \\
\hline
\end{tabular}

1/Time required for pit to begin boiling on loss of cooling is $4.1 \mathrm{hrs}$. Assume 63 hours over weekend when pit is not under human survelilance. Probability of fatlure in this time window computed as: $P=(63-4.1) / 720$.

2/Time required for pit to begin bolling due to fault vil ieft open is 5.2 hrs. Probability of human survefllance fallure computed as: $P=(63-5.2) / 720$.

$3 / T$ ime required for pit to begin boiling due to faults $v 12$ or v10 left open is $6.1 \mathrm{hrs}$. Probability of human survetilance
failure computed as: $\mathrm{p}=(63-6.1) / 7_{20}$. 
TABLE J8 (CONT'D)

FAILURE DATA - SPENT FUEL PIT FAULT TREE EVALUATION

\begin{tabular}{|c|c|c|c|c|c|c|}
\hline System/Component & Fallure Mode & Fallure Rate & $\begin{array}{c}\text { Fautit } \\
\text { Duration } \\
\text { Time } \\
\end{array}$ & $\begin{array}{l}\text { Probability } \\
\text { of Fallure }\end{array}$ & $\begin{array}{l}\text { Error } \\
\text { Factor }\end{array}$ & Remarks \\
\hline Valve $v 13$ & Left Open (Human) & $1 \times 10^{-2} / \mathrm{act}$ & $2.7 \mathrm{acts} / \mathrm{yr}$ & $2.7 \times 10^{-2}$ & 3 & Initiating event \\
\hline Valve V6 & Left Open (Human) & $1 \times 10^{-2} / a c t$ & 2.7 acts/yr & $2.7 \times 10^{-2}$ & 3 & Initrating event \\
\hline Valve V7 & Left Open (Human) & $1 \times 10^{-2} / d$ & & $1 \times 10^{-2}$ & 3 & Inttiating event \\
\hline Pump P2 & Falls to Run & $3 \times 10^{-5} / \mathrm{hr}$ & 3240 hrs & $9.7 \times 10^{-2}$ & 10 & $\begin{array}{l}\text { Initiating event; fails } \\
\text { to run in time between } \\
\text { malntenance acts }\end{array}$ \\
\hline Human Surveill ance & Falls & $6.8 \times 10^{-2} / \mathrm{d}$ & & $6.8 \times 10^{-2}$ & 10 & $\begin{array}{l}\text { Computed from time } \\
\text { interval when there is } \\
\text { no ptt survell lance I/ }\end{array}$ \\
\hline Valve Vi4 & Left Open (Human) & $1 \times 10^{-3} / \mathrm{act}$ & 12 acts $/ y r$ & $1.2 \times 10^{-2}$ & 3 & $\begin{array}{l}\text { Inftfating event; human } \\
\text { error with recovery }\end{array}$ \\
\hline Valve v9 & Left Open (Human) & $1 \times 10^{-3} / \mathrm{act}$ & $12 \mathrm{acts} / \mathrm{yr}$ & $1.2 \times 10^{-2}$ & 3 & $\begin{array}{l}\text { Initlating event; human } \\
\text { error with recovery }\end{array}$ \\
\hline Human Survefllance & Fails & $5.6 \times 10^{-2} / \mathrm{d}$ & & $5.6 \times 10^{-2}$ & 10 & $\begin{array}{l}\text { Computer from time } \\
\text { interval when there is } \\
\text { no pit survellilance 2/ }\end{array}$ \\
\hline
\end{tabular}

1/Time required for pit to begin bofling due to initiating events is $14.3 \mathrm{hrs}$. Probability of human survelliance fallure computed as: $P=(63-14.3) / 720$.

$2 / T i m e$ required for pit to begin bofling due to initiating events is $22.9 \mathrm{hrs}$. Probability of human survelllance failure computed as: $P=(63-22.9) / 720$. 


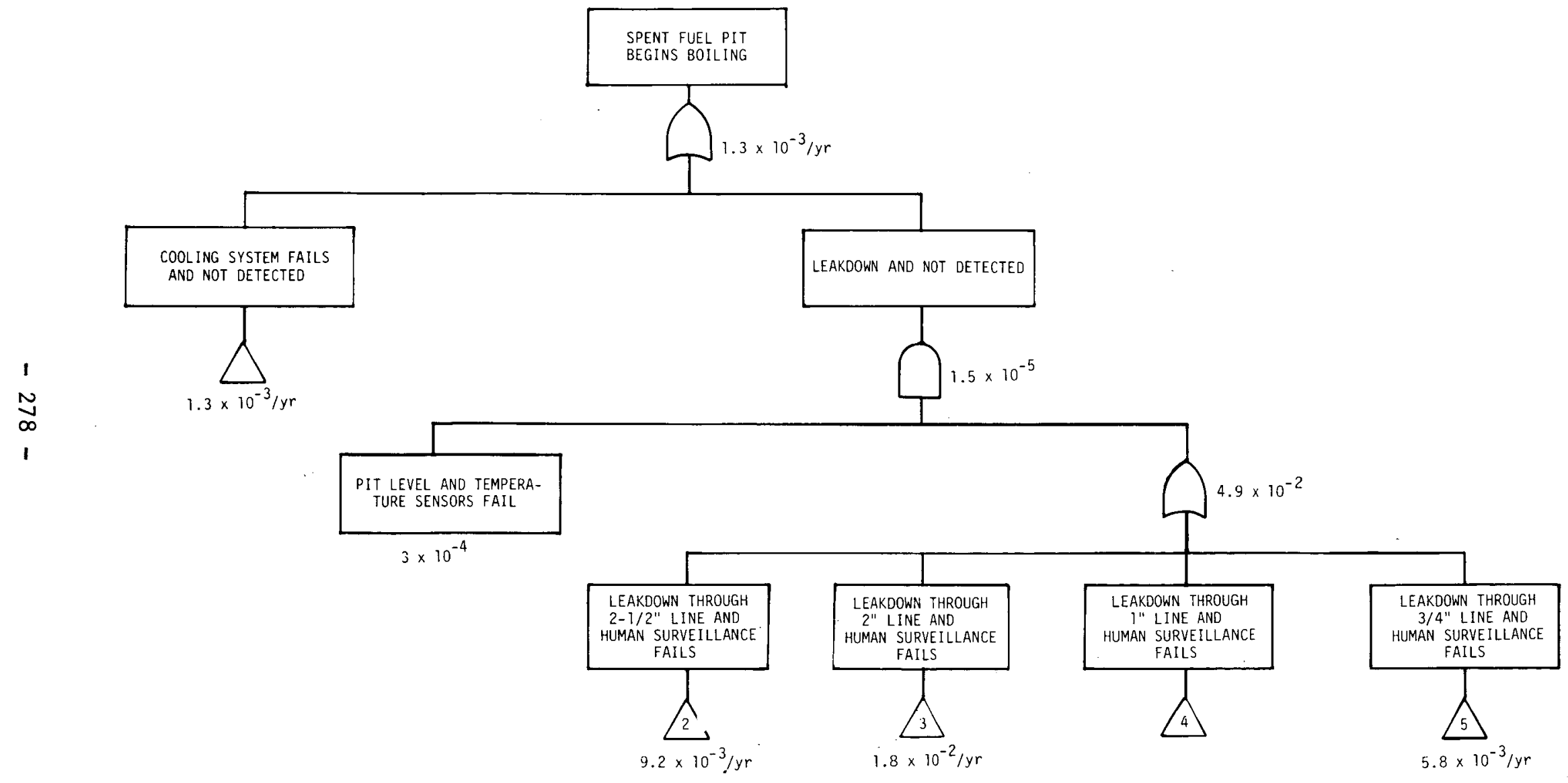

Figure J9. Fault tree for boiling of spent fuel pit 


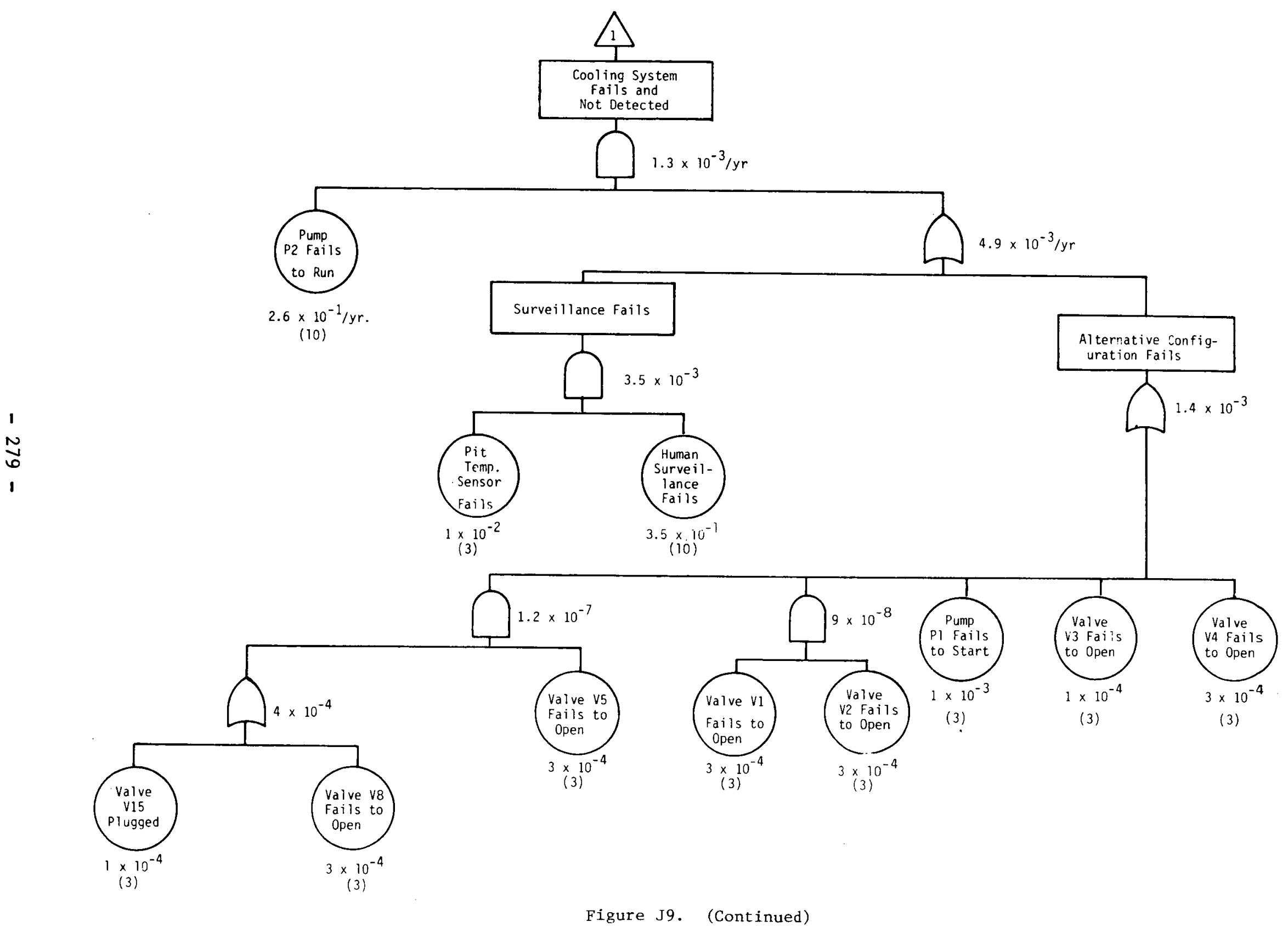



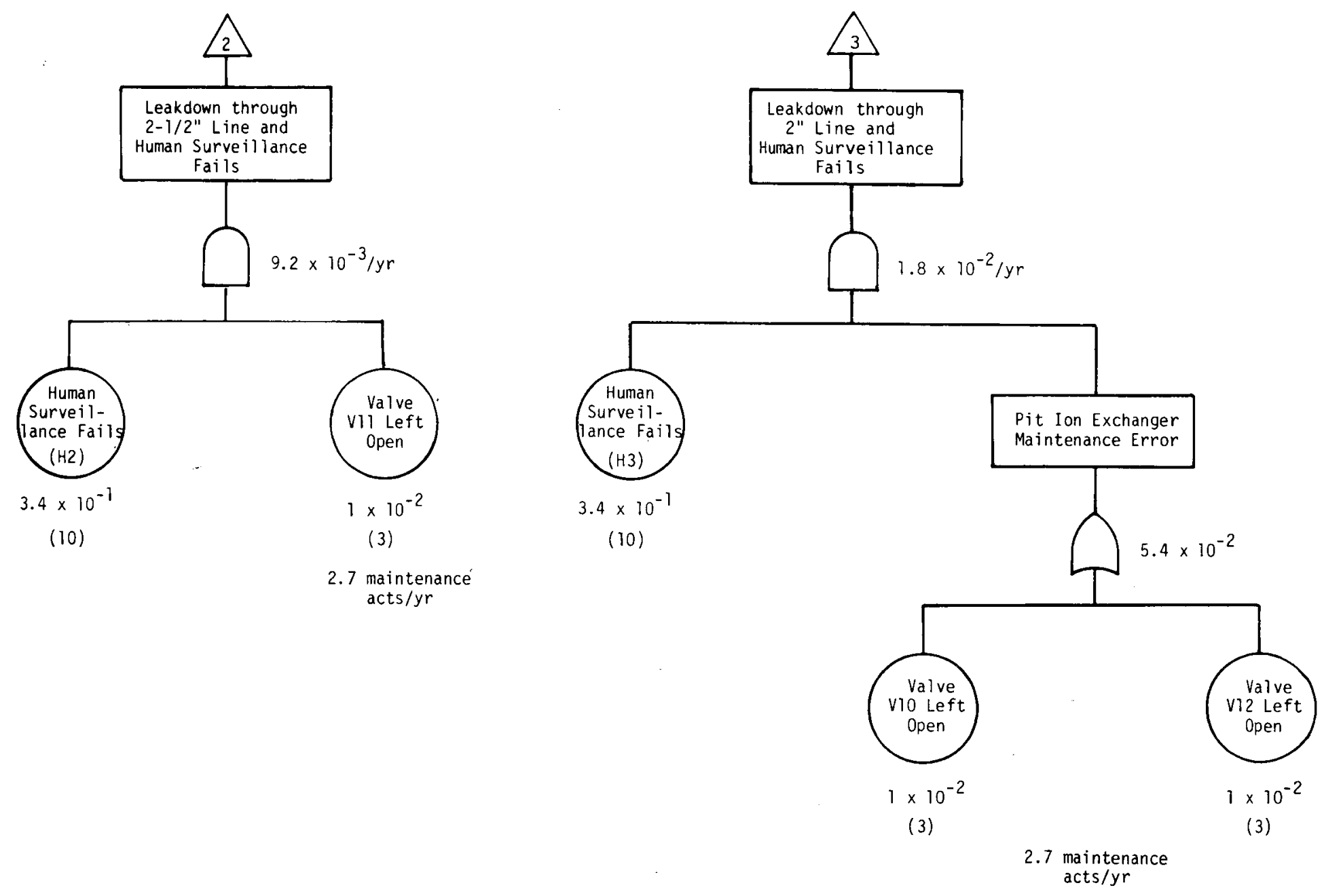

Figure J9. (Continued) 


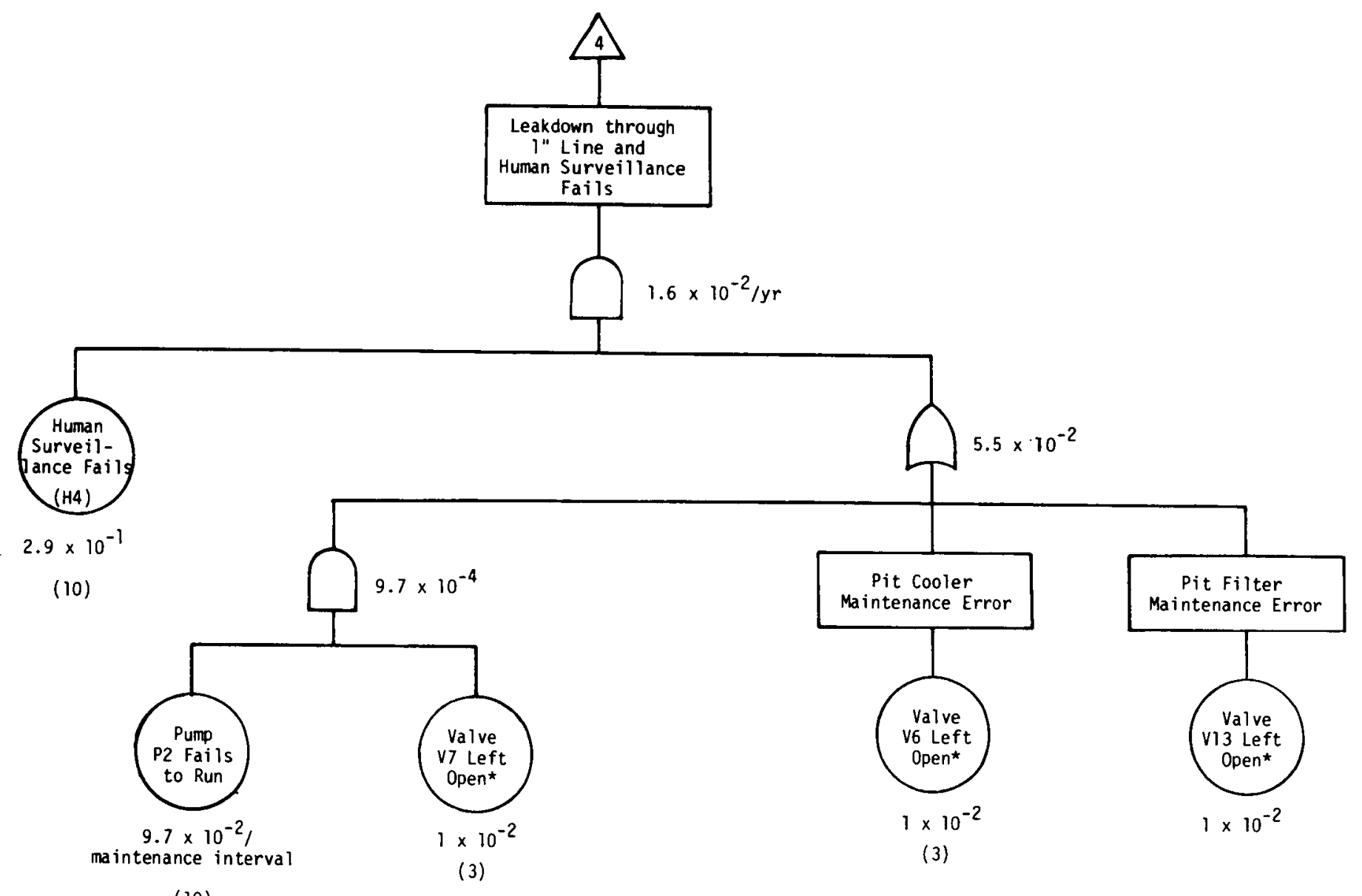

$\star 2.7$ Maintenance acts/yr.

Figure J9. (Continued) 


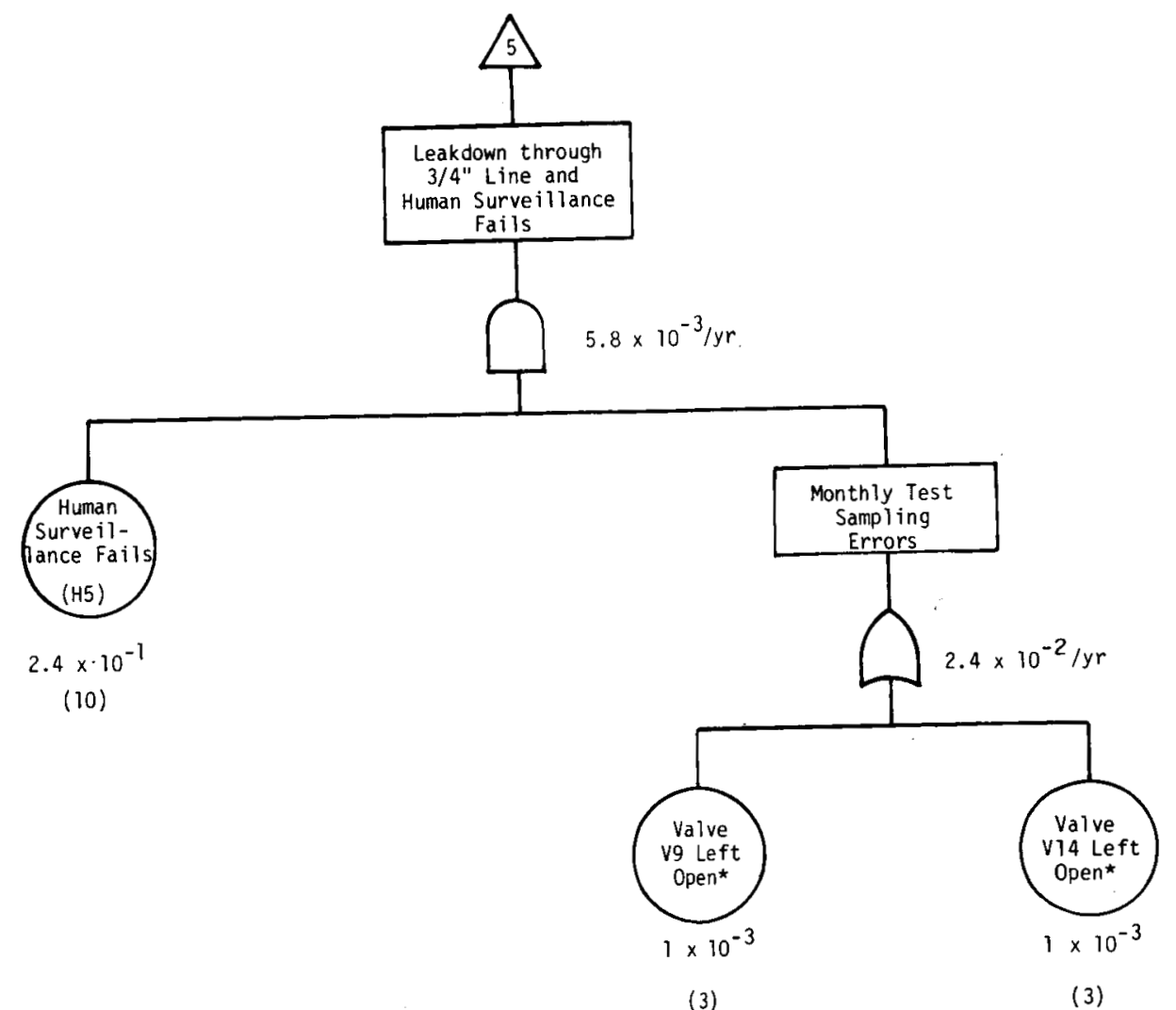

*12 Sample Tests/yr.

Figure J9. (Continued) 
The example quantification uses the conservatively estimated times for the water to heat to boiling, yiven an initiating event. Based on this example, it is apparent that the sequences involving siphoning will not contribute to the distribution of the top event, since they are at least two orders of magnitude less probable than sequences involving direct loss of pit cooling. Thus, the model uses only Figure $\mathrm{j} 9 \mathrm{~b}$ to evaluate the probability of pit boiling. The example quantification is based on a yearly assessment of accident probability.

Details of the example quantification are shown in Table J8. The failure rates and error factors of Table $J 8$ were obtained from WASH-1400,(1) Appendix III, except as otherwise noted in this table.

Point estimate evaluations are displayed on the fault tree, Figure J9, along with the error factors for the basic data. Most of these data are self-explanatory, with a few exceptions:

- In Figure J9b, the top level fault tree, the failure of the pit sensor systems was assumed as the lognormal median between an assumption of complete coupling between calibration of the level and temperature sensors $\left(1 \times 10^{-2}\right)$, and independence of these calibrations $\left(1 \times 10^{-2}\right)\left(1 \times 10^{-2}\right)=1 \times 10^{-4}$ Thus,

$$
\begin{aligned}
P & =\left(\left(1 \times 10^{-2}\right) \quad\left(1 \times 10^{-4}\right)\right)^{1 / 2} \\
& =1 \times 10^{-3}
\end{aligned}
$$

with error factor ten.

- In Figure J9b no credit was given for repair or pumps or valves in the four hours between the mutual failure sequence and pit boiling. The WASH-1400 data on repair times for pumps and valves indicates that the probability of not completing repair is greater than 0.8 for these components. 
- In Figures $39 \mathrm{c}$ through $\mathrm{J9e}$, the probability of an initiating event was calculated as the basic probability of a fault given an act times the number of acts per year. For instance, the probability of leaving valve VII open after maintenance is estimated to be $1 \times 10^{-2}$.

There were estimated to be 2.7 maintenance acts per year (from the average time between maintenance acts of 4.5 months given in Appendix III of WASH-1400). So the probability of an initiating event due to this fault was estimated to be:

$$
\begin{aligned}
P & =(2.7 \text { acts } / \text { year }) \quad\left(1 \times 10^{-2} / \text { act }\right) \\
& =2.7 \times 10^{-2} / \text { year. }
\end{aligned}
$$

- As discussed in Table J8, the probability of failure of human visual surveillance was estimated on the basis of a time window in which visual surveillance was assurned not to occur. An error factor of 10 was assigned to account for the large degree of uncertainty in this estimate. 


\section{APPENDIX K}

RELEASES FROM THE PRIMARY SYSTEM, INITIATING EVENT OF LOCA (DBA) LIQUID PATHWAYS

The purpose of this Appendix is to access the potential for radioactive release during one class 8 accident at a PWR using the VEPCO Surry Power Station as the typical plant. The analysis identified potential sources of radioactive releases and estimates the rates of release associated with each potential source. This section also assesses the capability of the waste disposal systems to adequately contain and process the released materials.

One Class 8 accident is defined as a Design Basis Accident (DBA) involving a double-ended displacement pipe rupture where all Engineered Safeguard and Containment Systems function properly. The radioactive release conditions investigated are those associated with excess leakage that does not constitute an engineered safeguard or contaiment system failure.

When a loss of coolant accident occurs, the reactor coolant flows from the pipe rupture into the contaiment area where it drains into the containment sump. Within seconds following the accident, Safety Injection System (SIS) water from the accumulators and the refueling water storage tank is injected into the Reactor Coolant System to rapidly make up water loss in the reactor core. The boron content of the initial charge of make up water fron the refueling water storage tank is increased to aid in controlling reactivity by injecting water through the boron injection tank.

The Containment Spray System, which is designed to control the buildup of containment pressure by spraying chilled water within contaiment, is activated 
by an increase in containment pressure due to the release of the primary coolant. Containment spray water also collects in the containment sump along with the spilt primary coolant. Long-term cooling is accomplished by switching to a recirculation mode where the accumulated water in the containment sump is reinjected into the Reactor Cooling System by the SIS to cool the core. Some of the water from the containment sump is also cooled and recirculated through the recirculation spray headers.

Leakage from any of the Coolant Circulation Systems within the confines of containment does not constitute an increase in the risk of radioactive release. Therefore, this analysis has concentrated on identifying increases in the flow of radioactive fluid drainage that could result during the functioning of the Engineered Safeguard Systems in a Class 8 accident that would constitute an increase in radioactive release in the non-containment areas of the plant. The types of faults investigated were sampling and test valves left open following maintenance, valve and pump leaks, sump pump failures, and the reverse flow of radioactive fluids into vented storage containers. The seriousness of the failure is identified in terms of the amount, or the rate, or radioactive fluid accumulation in the non-confinement areas of the plant.

Open Sampling Valve

First, a review of open sampling valves was conducted to detemine if there are any sampling valves that would only be charged when the SIS was either operational, or under test, and therefore could possibly be left open undetected during norinal operations of the power plant. In order to assure that all of the sampling valve candidates were identified, the numerous sampling systems were investigated to determine where they originated, their routing, and where 
they terminated. Sampling valves in the reactor coolant, auxiliary and emergency systems that are used to monitor primary and secondary system process fluids and gases were studied. Most of these samplings which are used to provide data from which the performance of the station, equipment and systems are determined are taken in the auxiliary building sampling room. However, a few samples are taken in the turbine building Unit No. 1 and Unit No. 2.

Sampling lines originating in containment but teminating in the auxiliary building may be directed to either a purge line or to the auxiliary building sampling sink. Sampling lines from points outside the containment but inside the auxiliary building also discharge into the auxiliary building sampling sink with the exception of the liquid waste evaporation sample which requires heat tracing and is, therefore, taken at the source. Turbine building sampling lines discharge into one of the building sampling sinks. High temperature samples also pass through sampling coolers and are manually throttled and can be directed to either a purge line or to the sampling sink. Samples entering the sampling sinks are directed into their respective floor drains and building sumps where they combine with other waste fluids.

This preliminary analysis conducted using the functional diagrans and drawings contained in the VEPCO Surry Power Station FSAR did not identify any sampling valves that were not charged during normal power plant operation. For example, if the local sample valve in the pump test line for the low head safety injection pump was left open it would drain off the refueling water storage tank water and activate the lower water indicator and alarm. Therefore, should any of these sampling valves in the SIS be inadvertently left open, a continuous flow would be observed in the sampling rooms, or at the sampling valve for those cases where the valve is located at the equipment as well as activate low water indicators and alarms. 
However, the boron injection tank does present some potential for a sampling valve to be inadvertently left open. The factor that contributes to this accident potential is that the boron injection tank is isolated from the SIS by parallel valves on each end of the tank so that the boron injection tank contents can be recirculated and maintained at a temperature greater than $130^{\circ} \mathrm{F} . *$

Valves in the boron injection tank recirculation system are controlled by the safety injection signal. Under normal plant operations these valves are held open. When safety injection is called for these valve close. They also fail closed. Should the boron recirculation valves fail closed or if the safety. injection signal is lost, the boron injection tank would be left unpressurized. Under these conditions, it is possible that one of the two sampling valves located at the boron injection tank could inadvertently be left open resulting in a high pressure discharge when the safety injection charging pump comes on during a Class 8 accident. Therefore, should a LOCA occur there is the possibility that as much as 385 gallons per minute of charging fluid would be discharged from an open 3/4" sampling valve. Should the safety injection charging pumps be turned off the low head safety injection pumps would provide sufficient pressure to maintain a discharge of waste fluid of as much as 165 gallons per minute. However, based on engineering judgrnent the probability for both of the valves to fail at the same time is expected to be extremely low.**

Valve Leaks

Next, valve leakages were reviewed. All valve stems are provided with drain lines and designed to provide maximum leakage of less than 1 cc per hour *FSAR $6.2 .2-17$

**Refer to Table K2, for flow data. 
per inch of stem diameter*. These leak-offs are in turn directed to the Waste Disposal System through their respective building surnps. Should there be a catastrophic seal failure in one of the nomally closed SIS valves, when the valve is actuated during a DBA the charging fluid could be discharged through the valve stem leak-off. It is possible that the flow of charging fluid though the leak-off into the building sump following a catastrophic seal failure may exceed the discharge capability of the building sump pump. The flow rate for a one-half inch leak (shown in Table K2) indicates the maximum excess flow expected should this type of failure occur. During recirculation with the low head safety injection pumps operating the flow would be reduced to less than one-half of the maximum flow.

\section{A Cumulative of Waste Fluids}

Waste fluids entering building sumps are transfered by sump pumps to either the high level waste or low level waste drain tanks depending on the reactivity level of the waste fluid. Approximately 4000 gallons of high level waste and 6000 gallons of low level waste can be stored prior to processing and disposal. The rated capacity of the various sump pumps and the time which it would take each pump operating at rated capacity to fill the waste drain tanks is shown in Table $\mathrm{K} 1 . * *$

The auxiliary building pump is the collector for the largest potential volume of waste fluids of the six building sump areas shown in Table k1. This sump has a rated capacity to handle up to 50 gallons of waste fluids per minute. Should the flow of waste fluids into the sump exceed 50 gallons per FFSAR $6.2 .2-23$

$\star \star$ FSAR Section 9.7 and 11.2 


\section{TABLE K1}

\section{SUMP PUMP CAPACITY*}

Area

Auxiliary Building

Fuel Building

Safeguard Area

Component Cooling Heat Exchange

Reactor Containment

Incore Instrument Room**

$$
\text { Capacity }
$$

GPM

50

25

25

25

25

10
Time to Fill

(Hours)

$$
3-1 / 2
$$

7

7

7

7

$N / A$

*FSAR Table 9.7-1

**The incore instrument room sump pump feeds into the reactor containment sump 
minute; excess waste fluids will accumulate in the sump. The rate at which waste fluids could escape from the SIS into the building sump will vary as a function of the size of the leak, and the pressure and temperature of contained fluid.

\section{Sump Pump Failure}

A11 sump pumps in the reactor containment sump; the safeguards area sump, and the auxiliary and fuel building sumps are dual redundant. These floatactivated pumps operate on an alternating cycle where the off-cycle pump is also activated by a backup float switch in case the on-cycle pump should fail to start. Containment sump pumps are also operated in conjunction with the opening of motor-operated isolation valves in the drain lines when these pumps and valves are removed from service should a containment overpressure condition arise. Should the auxiliary or fuel building sump pumps both fail, the below grade areas in these buildings would be gradually flooded. The probability of both sump pumps failing would be approximately $10^{-6}$.*

\section{SIS Pump Characteristics}

In order to evaluate the magnitude of potential radioactive leakage from the SIS in the recirculation mode, a review of basic pump characteristics was made. The SIS uses centrifugal pumps for the generation of pressure head as well as the moving of liquids. The pressure head produced by these pumps is a primary parameter in determining the leakage rate for particular effective leak size independent of this type of leak. The three parallel safety injection charging pumps are an example of pumps designed to operate against a large pressure head. These pumps may be used singularly, in pairs, or three at a

*WASH-14001 
time (according to demand) to inject a high boron concentration fluid into the primary coolant system to control increases in radioactivity and to make up for small break loss of coolant fluid losses. The two parallel low head safety injection pumps operated singularly or as a pair are an example of pumps designed primarily to move a relatively large volume of fluid. These pumps are used to make up for large coolant losses in a large break LOC accident when the Primary Coolant System has been depressurized.

The Safety Injection Charging Pumps are designed to produce large pressure heads generally operate at higher speeds and are subject to relatively large pressure head variation as a function of flow demand. For example, although the head at 50 percent of rated capacity may be up to twice the rated head, a 25 percent increase in demand capacity may reduce the discharge head to less than 50 percent of rated head.

Although the VEPCO Surry Power Plant FSAR does not specify pump speed, the pump parameters specified in Table $6 \cdot 2 \cdot 2 \cdot 2-3$ of the FSAR do indicate scoping parameters for the safety injection charging pumps. These parameters are:

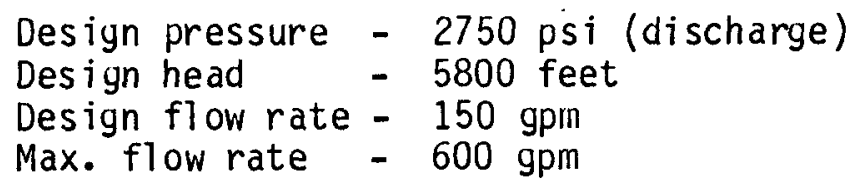

The following assumptions have been made:

- The maximum flow rate specified in this table is assumed to be the flow rate that would occur with a guillotine rupture of the discharge pipe connection at the pump.

- The design pressure of the pump at 2750 psi is 515 psi greater that the operating pressure of the primary coolant system. The design flow rate of the pump is assumed to be the discharge flow rate of the charging fluid 
into the primary coolant system for sinall break LOC accident when the LOC flow rate is not large enough to reduce the operating pressure of the primary coolant system below its specified operating pressure of 2235 psi.

The performance estimating technique introduced in this analysis uses basic pump characteristic relationships to roughly estimate the effect of pump capacity on discharge pressure. These estimated pump characteristics are then used to estimate fluid flow in the safety injection charging pump system and therefore derive a more precise estimate of the leakage rate from leaks in the ECCS systen.

Although the head-capacity characteristics for a centrifugal pump can vary greatly as a function of pump design, all the various characteristics have one feature in common, the pressure head will decrease with an increase in demand capacity after the design conditions are exceeded. The two pump design data points specified in the FSAR are shown plotted on Figure Kl. These data points are the design flow rate of $150 \mathrm{gpm}$ at a design pressure of $2750 \mathrm{psi}$ and a maximum flow rate of $600 \mathrm{gpm}$ at a discharge pressure assumed to be zero psi. For this initial calculation, a simplified pump head curve was constructed using these points and is shown in Figure K1. Because of the lack of more explicit data, these data points were connected by a straight line to reflect the inverse relationship between discharge pressure and pump capacity for pump capacities greater than the design flow rate.

The draw-down characteristics shown in this figure provide a basis for evaluating the combined effect of injection fluid flow and leakage on discharge pressure. 


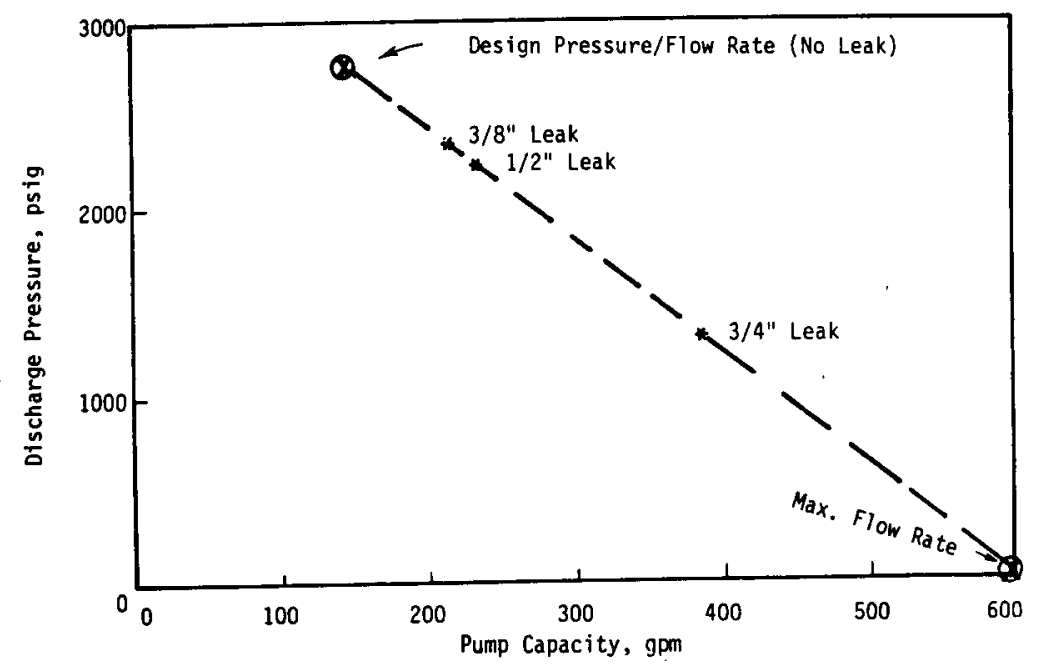

Figure K1. Estimated characteristics of Surry safety injection pumps

TABLE K2

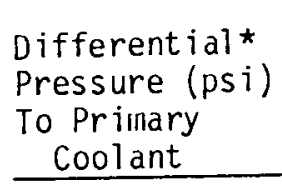

515

150

4

$-0-$

0
2750 *

2385

2239

2235

1350
Fluid Flow (gpm)

\begin{tabular}{cccc} 
To Priniary & $3 / 8^{\prime \prime}$ & $1 / 2^{\prime \prime}$ & $3 / 4^{\prime \prime}$ \\
Coolant & Leak & Leak & Leak \\
\hline
\end{tabular}

150 *

81

128

13

220

$-0-$

0 
The Low Head Safety Injection Pumps designed to produce relatively large flow rates generally operate at lower speeds. Therefore, these pumps are less subject to large pressure head variation as a function of flow demand. For example, the head at less than rated capacity may rise only slightly above the head at rated capacity and a 25 percent increase in demand capacity may reduce the discharge head less than 20 percent. However, the maximum flow rate will probably be sharply curtailed to some level dependent on the internal capacity of the pump (i.e., size of the pump's displacement).

The VEPCO Surry Power Plant FSAR also does not specify pump speed as part of the pump parameters listed in Table $6 \cdot 2 \cdot 2 \cdot 2-3$ for the low head safety injection pumps. The parameters listed are:

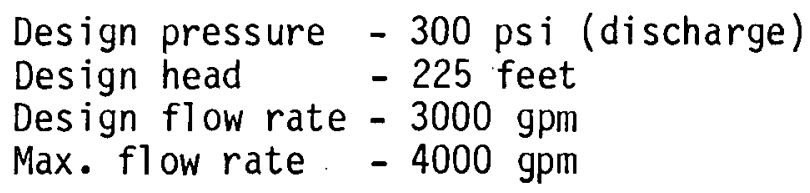

These data illustrate the relationship between the relatively low design pressure and the relatively large design flow rate of pumps designed to move liquids.

Leakage Rates in Small Break LOC Accidents

For the small break Loss of Coolant accident case, the fluid from the Safety Injection charging pump flows through a parallel path: Either into the primary coolant system, or into the auxiliary building sump through the parameterized leak. The flow rate of the charging fluid into either of these systems is determined by the differential pressure between the charging pump discharge pressure and the pressure existing in the primary coolant system or at the auxiliary building sump. Without a leak, the charging fluid is assumed to flow at the "design flow rate" into the primary coolant system. When a leak 
occurs, the discharge pressure of the SICS charging pump is reduced inversely proportionate to the leak flow rate as described in Figure $\mathrm{K} 1$. As the size of the leak is increased and its flow rate is increased, the fluid flow through the lead will "draw down" the discharge pressure until the discharge pressure and the pump capacity reach an equilibrium as defined by the data presented in Figure 10 .

The flow rate of the injection fluid is calculated using Bernoulli's equation:

$$
h=f \frac{L}{d} \quad \frac{v^{2}}{2 g}
$$

where

$$
\begin{aligned}
& h=\text { pressure head, feet } \\
& f=\text { friction coefficient } \\
& L=\text { length of pipe, feet } \\
& d=\text { diameter of pipe, feet } \\
& v=\text { flow velocity, gpm } \\
& g=\text { gravitational acceleration }
\end{aligned}
$$

assuming for simplicity

$$
f \frac{L}{d 2 g} \sim \text { constant }(k)
$$

then

$$
h=k v^{2} \text { and } k=\frac{515}{(150)^{2}}=.0229
$$

where

$$
\begin{aligned}
& h=515 \mathrm{psig} \\
& v=150 \mathrm{gpm} \text { (design flow). }
\end{aligned}
$$

The flow rate of the leakage is calculated using the potential mass flow rate for estimating critical flow in short pipes, nozzles, and orifices for saturated water flow. (48)

The reduced charging pump discharge pressure, in turn, affects the flow rate of the charging fluid leaking from the charging system. When the discharge pressure of the SIS charging pump drops below the operating pressure of 
the primary coolant system, flow into this system halts (a check valve blocks reverse flow). An estimate of the potential waste fluid flow in a small break LOC accident for single pump operation. is shown in Table K2. The differential pressure estimated for both of the parallel paths is included in the Table for each leak condition.

It is concluded that the sampling and test valves in the ECCS system are pressurized and cannot be left inadvertently open without drainage of either the refueling water storage tank or the boron storage tanks. Draw down on either of these tanks will activate indicators and alarms. Therefore, the probability of this type of release occurring should be very low. In addition, the SIS valves are designed to very close tolerances and valve stem leakage is normally expected to be minimal. However, a catastrophic seal failure in one of the nomally closed SIS valves when it is activated during an emergency. could pressurize the valve stem leak-offs and cause flows of up to 220 gallons per minute when the SIS charging pump is activated and approximately 100 yallons per minute when the low-head SIS pumps are activated. The sump pumps do not have the capacity to drain off all the fluids that may be released should an SIS valve seal catastrophically fail during safety injection. Therefore, during the recirculation mode radioactive fluid could build up in the auxiliary building at a rate of approximately 50 gallons per minute.

It should be noted that the catastrophic scenario discussed above presupposes a LOCA event. Given the probability of a large LOCA at $10^{-4}$ /reactor year, taken from WASH 1400, it can be concluded that the risk involving a significant radioactive release from the SIS in recirculation will be small when compared to the class 9 accidents, due to its relatively low probability and low release terms. 
APPENDIX $L$

LARGE BORE PIPE RUPTURE PROBABILITIES

AS APPLIED TO A STEAM LINE BREAK

\section{L.1 Introduction}

The steam line break downstream of containment and upstream of the main isolation valve, in a PWR is of special interest in the BNL-LWR Risk Assessment Program because of the current problems with degraded steam generator tube and possible ruptures. This section will explore the probability of a steam line break with the thought of applying it to the scenario of STGR/SLB. This appendix will also outline the problem of large bore pipe rupture, and give details of 4 contributing accidents. Of specific interest will be the steam piping outside of the contaiment between the steam generators and the main steam isolation valve. The conditional probability of a steam generator tube rupture initiated by a steam line break or safety valve failure in this section of line will also be briefly discussed.

\section{L.2 Literature Search}

A review of the literature reveals nothing on the specific section of piping of a PWR, but many dissertations on the general topics of piping and steam line failure probabilities. These can be divided into two major groups. Those based on actual experience and those based on other papers and on data supplied by others.

Table LI gives a summary of the probabilities given for large pipe ruptures based on experience most used by other authors. Note that WASH-1400 can be the 
source of various estimates (and is used by different authors to come up with various probabilities.) The values fall in the range of $10^{-4}$ to $10^{-5}$ events per reactor year, for catastrophic failures in large bore steam pipes. Failure rates which include smaller sizes and noncatastrophic failures (leaks or small cracks) are yiven in the range of $10^{-2}$ to $10^{-4}$ failures per plant year by the same sources.

Table L2 gives a summary of probabilities cited in papers based on analysis of other papers, including some strictly theoretical analyses. They result in probabilities of the order of $10^{-6}$ events per plant year but are generally less realistic because they fail to include all failure mechanisms in their discussion (i.e. operator error, corrosion, errosion, etc.) In particular, Reference 49 deals strictly with undetected flaws and their consequent growth to failure by stress and themal cycling.

Some papers(50), dealing with specific sections of piping such as the reactor coolant system, cite probabilities in the order of $10^{-8}$, but these are strictly theoretical treatises using fracture mechanics to predict failure at specific welds. They ignore all other modes of failure at other locations or components.

In all papers addressing the probability of large bore rupture in nuclear primary systems one will find the statement made that there have been no catastrophic failures to date in land based stations during power operation. Some, References $49 \& 1$, will also include military experience. These papers will generally conclude that based on one event per 200 plant years (approximately, depending on when paper was written), the "upper bound" for the event of interest is less than or equal to $10^{-2}$ events per plant year.

The literature is therefore of limited value in supporting any conclusion 
TABLE L1

PROBABILITIES OF LARGE BORE PIPE RUPTURES

BASED ON ACTUAL EXPERIENCE

\begin{tabular}{|c|c|c|c|c|c|}
\hline Source & Ref. & Nuc. & $\begin{array}{l}\text { Non } \\
\text { Nuc. }\end{array}$ & $\begin{array}{l}\text { Events per } \\
\text { Plant Year }\end{array}$ & Comment \\
\hline WASH- 1400 & 1 & $x$ & & $1 \times 10^{-4}$ & $\begin{array}{l}\text { From tabie III 6-9 (pipe > 6") } \\
\text { LOCA initiating median (range } \\
10^{-3} \text { to } 10^{-5} \text { ) }\end{array}$ \\
\hline WASH- 1400 & 1 & $x$ & & $\left(8.8 \times 10^{-7}\right)$ & $\begin{array}{l}\text { (Events per section year) } \\
\text { given in table III } 2-1 \text { (pipe } \\
>3^{\prime \prime} \text { ) used in ref. } 20 \text { to arrive }\end{array}$ \\
\hline & & & & & $\begin{array}{l}\text { at estimate of } 1.5 \times 10^{-5} \text { per } \\
\text { plant year, for primary circuit. }\end{array}$ \\
\hline GEAP 4574 & 52 & & $x$ & $1 \times 10^{-4}$ & $\begin{array}{l}\text { U.S. Fossil plant piping } \\
\text { experience using data from } \\
\text { WASH } 1400\end{array}$ \\
\hline $\begin{array}{l}\text { PHILLIPS \& } \\
\text { WARWICK }\end{array}$ & 53 & & $X$ & $2 \times 10^{-5}$ & U.K. Vessel experience \\
\hline
\end{tabular}

TABLE L2

PROBABILITIES OF LARGE BORE PIPE RUPTURES BASED ON ANALYSIS

Source Ref. Nuc. $\begin{aligned} & \text { Non } \\ & \text { Nuc. }\end{aligned}$

\begin{tabular}{|c|c|c|c|c|c|}
\hline $\begin{array}{l}\text { LINDACKERS \& } \\
\text { STOEBEL }\end{array}$ & 54 & & $x$ & $1.7 \times 10^{-6}$ & $\begin{array}{l}\text { Based on German pressure } \\
\text { vessel experience }\end{array}$ \\
\hline $\begin{array}{l}\text { HARRIS \& } \\
\text { FULLWOOD }\end{array}$ & 49 & $x$ & & $2 \times 10^{-6}$ & $\begin{array}{l}\text { Prediction for first } 10 \\
\text { after initial UT or RT }\end{array}$ \\
\hline $\begin{array}{l}\text { HARRIS \& } \\
\text { FULLWOOD }\end{array}$ & 49 & $x$ & & $3.6 \times 10^{-6}$ & $\begin{array}{l}\text { Based on World Nuclear } \\
\text { Experience }\end{array}$ \\
\hline
\end{tabular}


since the range $10^{-2}$ to $10^{-6}$ for this particular event is too wide. The only consistancy found in the references that should be noted is that the severity factor, (i.e., the ratio of catastrophic failures to all failures), seems to be consistant for both pipe and pressure vessels (less than $5 \%$ in both References 52 \& 53). This ratio is based on non-nuclear experience. In addition, a cursory review of one LER file (component failures during test) reveals only one near-catastrophic failure in 27 , which tends to confirm this ratio.

Other failure data $(52,53)$ however, tends to show that there are more pipe failures of varying severity in nuclear plants than in conventional steam plants. Since much of the piping in nuclear plants is of higher quality, undergoes inore frequent inspection, and is subject to lower temperatures and pressures than most modern fossil fuel central station plants, one would expect the reverse to be true. The apparent discrepancy can be explained by the fact that in nuclear practice, all events which have a potential for affecting safe operation must be reported, consequently the LER files( 3 ) contain 1 " and smaller line failures (particularly at weldments to larger pipe). These pipes are not subject to any stricter inspection than in normal steam plant practice. In commercial central station plants, only those failures which affect the plants insurance are likely to be reported and so the data base is missing many "nuisance" failures when compared to nuclear plant experience. In summary, there are certain shortcomings in applying data from other industries except in a "supportive" role. 


\section{L.3 Presently used Value for Steam Line Break}

Sumnarizing the previous work done on the general subject of large bore steam pipe rupture, one can say that the consensus failure rate is in the range of $10^{-4}$ to $10^{-6}$ failures per plant year. If you take a weighted average of these values, the rate will be of the order at $10^{-5}$ failures per year. (This is similar to conclusion of table 15 in Reference 49). If one adheres strictIy to nuclear experience, however, we are guided to the more conservative $10^{-4}$ per plant year by References $55 \& 1$. The following analys is is based on the data given in References $55 \& 1$.

There are three main factors which enter into the assessment of failure rate of pipes from surveys of pipe failures an nuclear power plants (NPPs):

1. The period over which the survey was conducted.

2. The number of plants included in the survey.

3. The number and type of failures recorded.

Bush (55) and WASH-1400(1) provide the two most recent assessments of nuclear experience data on the probability of pipe failure.

The WASH-1400(1) study restricted itself to failures of pipes in plants which operated one complete year, (1972), and included 17 nuclear power plants of which 8 were PWR's and 9 BWR's. The number of failures were 3 and 8 in PWR's and BWR's respectively. An inspection of type and nature of pipe failures (WASH-1400, (1) Appendices III and IV, p. III-24) shows that all of them were innocuous failures, (none were double guillotine rupture or catastrophic).

Bush's(55) survey of pipe failure data was from 1970-1975. It included 126 failures from the pre-operational and operational phase of which 69 were in PWRs and 57 in BWRs. The number of reactor-years over which the failures accrued were 250 (reactor-years) approximately divided between BWRs and PWRs. Of 
the total 126 failures, engineering judgment exercised by Bush showed 8 of them, 4 in PWRs and 4 in BWRs, had substantial safety significance.

The failure data in the aforementioned surveys were of the 'time censored' form. Assuming that major fajlures occurred randomly in time and followed a Poisson process, maximum likelihood estimates of the failure rate, $\lambda_{2}$ (failures/reactor year) and $\lambda_{2}$ (failures/ft-year) are presented in Table L3. In this computation it is assumed that the average length of piping in a PWR or a BWR is 35,000ft. A conparison of the estimates of the failure rate, $\lambda_{2}$, derived from the two surveys shows certain interesting features. If all types of failures in Bush's(55) survey are included in the computation of failure rates then the results in comparison to WASH-1400 are of the same order of magnitude $10^{-5}$ (failures/ft-year). The fact that Bush's(55) survey spanned fram 1970 to 1975 while WASH-1400 had only 1972 failure data, and yet $\lambda_{2}$ is of the same order of magnitude lends some validity to this number. A comparison of "safety significant". or "catastrophic" failure rate cannot be done since none of the failures in WASH-1400(1) falls into this category.

For the BNL-LWR risk assessment program large breaks in the three divisions of the steam line of PWR is of special interest.: For the purpose of this BNL program, we may be tempted to use $\lambda_{2}=9.1 \times 10^{-7}$ (failure/ft-year), from. Table $L 3$, as an interim value subject to modification. The failure rate per year for the three divisions of the steam line are shown in Table L4. The lineal footages as shown in this table are approximate values for Surry Unit 1. When the values given in Table $L 4$ are used in this risk assessment for a steam line break inside of containment, or downstream of the isolation valve, the other probabilities involved are controlling, and the end result of the 
TABLE 1.3

FAILURE DATA AND FAILURE RATE ASSESSMENT OF PIPE FAILURES NNPS

\begin{tabular}{|c|c|c|c|c|}
\hline & & PWR & BWR & Combined \\
\hline \multirow{2}{*}{ Reactor-Years } & $\operatorname{Ref} 51$ & 125 & 125 & 250 \\
\hline & $\operatorname{Ref} 1$ & 8 & 9 & 17 \\
\hline \multirow{2}{*}{ Number of Failures } & $\operatorname{Ref} 55$ & $\begin{array}{l}69 \\
4^{\star}\end{array}$ & $\begin{array}{l}57 \\
4^{*}\end{array}$ & $\begin{array}{r}126 \\
8 *\end{array}$ \\
\hline & $\operatorname{Ref} 1$ & 3 & 8 & 11 \\
\hline \multirow{2}{*}{$\begin{array}{l}\text { Failure Rate } \rightarrow \lambda_{1} \\
\frac{\text { failures }}{\text { reactor-year }}\end{array}$} & Ref 55 & $\begin{array}{l}5.5 \times 10^{-1} \\
3.2 \times 10^{-2}\end{array}$ & $\begin{array}{l}4.6 \times 10^{-1} \\
3.2 \times 10^{-2}\end{array}$ & $\begin{array}{l}5.0 \times 10^{-1} \\
3.2 \times 10^{-2}\end{array}$ \\
\hline & $\operatorname{Ref} 1$ & $3.8 \times 10^{-1}$ & $8.9 \times 10^{-1}$ & $6.5 \times 10^{-1}$ \\
\hline \multirow{2}{*}{$\begin{array}{l}\text { Failure Rate } \rightarrow \lambda_{2} \\
\frac{\text { failures }}{\text { ft-year }}\end{array}$} & $\operatorname{Ref} 55$ & $\begin{array}{l}1.6 \times 10^{-5} \\
9.1 \times 10^{-7} \text { * }\end{array}$ & $\begin{array}{l}1.3 \times 10^{-5} \\
9.1 \times 10^{-7} \star\end{array}$ & $\begin{array}{l}1.4 \times 10^{-5} \\
9.1 \times 10^{-7} \star\end{array}$ \\
\hline & Ref 1 & $1.1 \times 10^{-5}$ & $2.5 \times 10^{-5}$ & $1.8 \times 10^{-5}$ \\
\hline
\end{tabular}

*Catastrophic or near-catastrophic failures 
TABLE L'4

FAILURE RATE PER YEAR FOR THREE DIVISIONS

OF STEAM: LINE OF A PWR.

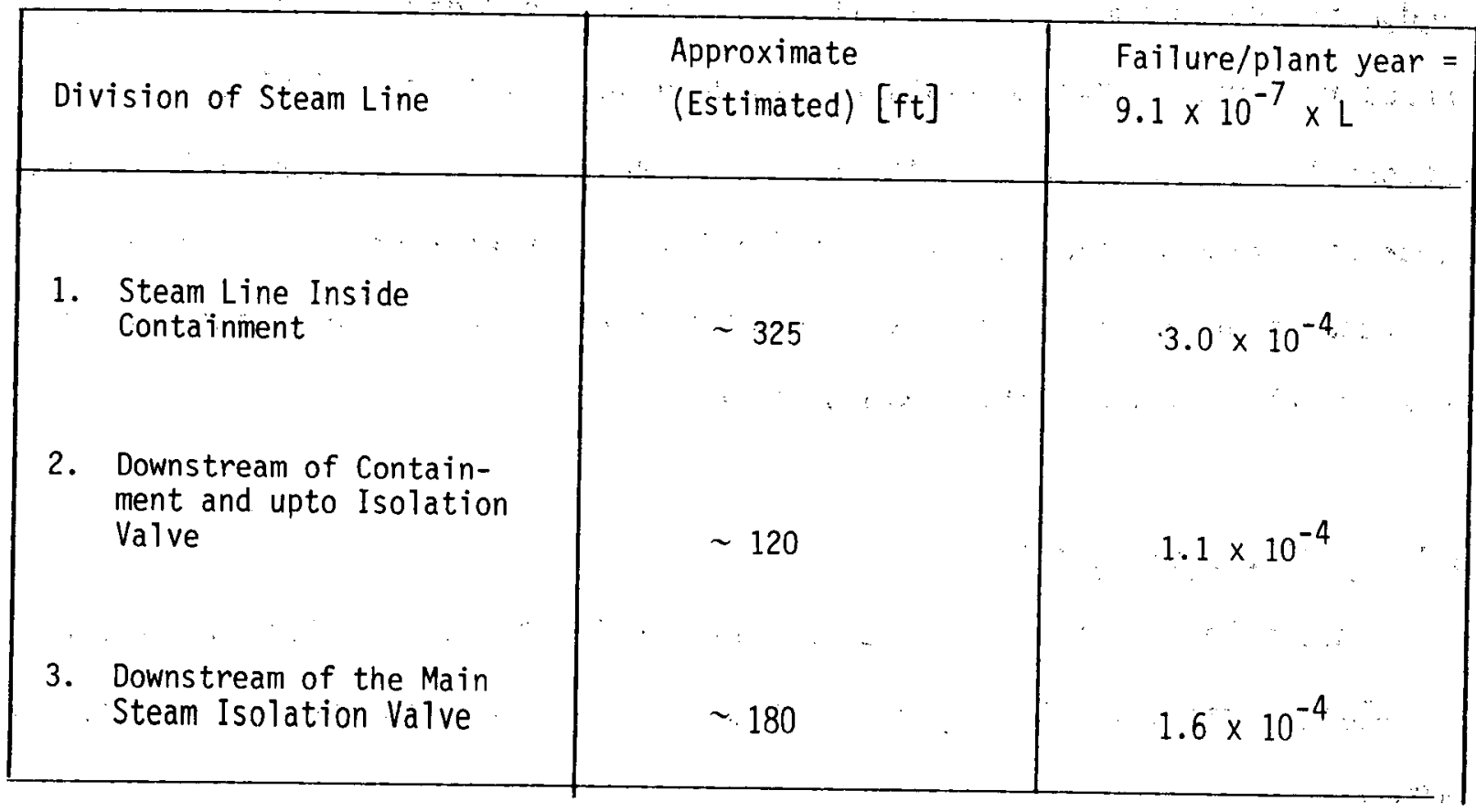


sequence calculations is of the order of $10^{-9}$ events per year or lower. In these cases the effect of the present inaccuracy of the steam line break probability is acceptable.

When used in the specific case of the steam line break downstream of containment and upstream of steam isolation valve, zone 2, given a dependent probability of steam generator tube rupture, it becomes dominant and must be examined more closely. In this sequence, where the probability of the steam generator tube rupture is initially estimated as $10^{-2}$, based on engïneering judyment, the sequence probability will be of the order of $10^{-6}$ to $10^{-4}$ per reactor year for a primary system blowdown outside of containment.

It should be noted that initial themal hydraulic calculations show that within a short period of time after the initial event, the pressure of the primary can be reduced and the release brought under control. For an evaluation of expected releases see section 3.5 .3 of this report.

\section{L3.1 Failures per Foot}

It should be noted that the use of "per foot" failure rates as applied here can lead to error. The major objections to this kind of averaging are as follows :

1. Failures in piping are more likely to occur at discontinuities (welds, joints, etc.) than in the pipe wall (see Table L5).

A more representative rate would be one per component, with a component being defined as anything which caused a discontinuity, but the true rate would be one which also included the effect of pipe length between discontinuities. 
2. The 'per foot' rate for an entire plant does not take into account the different design and specifications of pipe such as ASME class.

3. Errors can be introduced because the lengths cited are estimates, not actually measured.

4. The fact that certain systems having comparable pipe lengths are more prone to failure than others is completely masked by this gross averaging. For instance reference 2 shows that for PWR's the charging systems have a greater proportion of failures, while in BWR's the feedwater system is the leader.

5. When the "per foot" number is used, the probability of failure for any specific section is arrived at by dividing the fotage in that section by the total plant footage. The latter is so large compared to the section footage that the resultant is insensitive to errors in the footage, chosen for the section.

In summary, while a 'per foot' number may be used to show relative rates for similar design plants and systems based on size of plant (assuming that in a similar design, the number of components is proportional to the length of pipe), it should not be used to derive failure rates for any particular section of any given plant. It is used here by BNL only to illustrate the problems with its use.

\section{L.4. Selected Large Bore Pipe Ruptures Cases}

This section describes four selected accidents involving large bore pipes as an example of reported major failures. The accidents considered may not be the only ones which have happened in commercial nuclear power plants as a complete search of Licensee Event Report (LER) files has not yet been completed. 


\section{TABLE L5}

PROPORTION OF FAILURES AT VARIOUS LOCATIONS

IN PIPING SYSTEMS (PERCENTAGE OF TOTAL FAILURES)

\begin{tabular}{|c|c|c|c|}
\hline Source & $\begin{array}{l}\text { Failures at } \\
\text { Weld or } \\
\text { Heat Affected } \\
\quad \text { Zones } \\
\end{array}$ & $\begin{array}{l}\text { Failures in } \\
\text { Other Specific } \\
\text { Locations } \\
\text { (Discontinuities) }\end{array}$ & $\begin{array}{l}\text { Failures in } \\
\text { Pipe Wall } \\
\text { Between Joints }\end{array}$ \\
\hline $\begin{array}{l}\text { GEAP } 4574 \\
\text { (all failures) }\end{array}$ & 38 & 18 & 44 \\
\hline $\begin{array}{l}\text { GEAP } 4574 \\
\text { (ruptures) }\end{array}$ & 55 & 20 & 25 \\
\hline EPRI NP-438 & 54 & 6 & 40 \\
\hline $\begin{array}{l}\text { LER Data }(1) \\
\text { (Oct. } 4,1978)\end{array}$ & 63 & 12 & 24 \\
\hline
\end{tabular}




\section{L.4.1 Plant: H.B. Robinson Unit No. 2 (Carolina Power and Light Co.)}

Occurrence: April 28, 1970

Plant Status: There was no fuel in the reactor. Hot functional tests were going on and lift pressures of safety valves were being checked. Eight safety valves in main steam lines $A$ and $B$ had been tested satisfactorily. The main steam line isolation and stean bypass valves were closed. The secondary side was maintained at a pressure of 900 psi and a temperature of $530^{\circ} \mathrm{F}$. The valve SV1-4-C was being checked for lift pressure by means of a pneumatic device and the air pressure was being increased when the incident occurred. The set point of this valve supplied by the vendor was 1140 psi.

Incident Description: There was initial break below the weld in the tapered zone (schedule 40) of the 6" diameter branch line connecting the valve to the 24 " diameter main steam line. This is illustrated in Figure L1. The emitted steam for a brief period of time formed a fan jet with a fan angle of $80^{\circ}$ wide and was directed $45^{\circ}$ up from the horizontal. This was followed by a $360^{\circ}$ circumferential break almost $n$ the horizontal plane. Bending and tearing of the valve assembly occurred about an axis perpendicular to the exhaust pipe. The safety valve apparently rotated as the pipe tore across its section. This gave rise to a vertical column of steam which rose an estimated $150 \mathrm{ft}$. The force of the steam jet caused the expulsion of the whole valve assembly which first struck the supports of the steam line, rebounded, and caused damage to auxiliary boiler stacks and its support before coming to rest in the turbine building. Seven men suffered steam burns. 


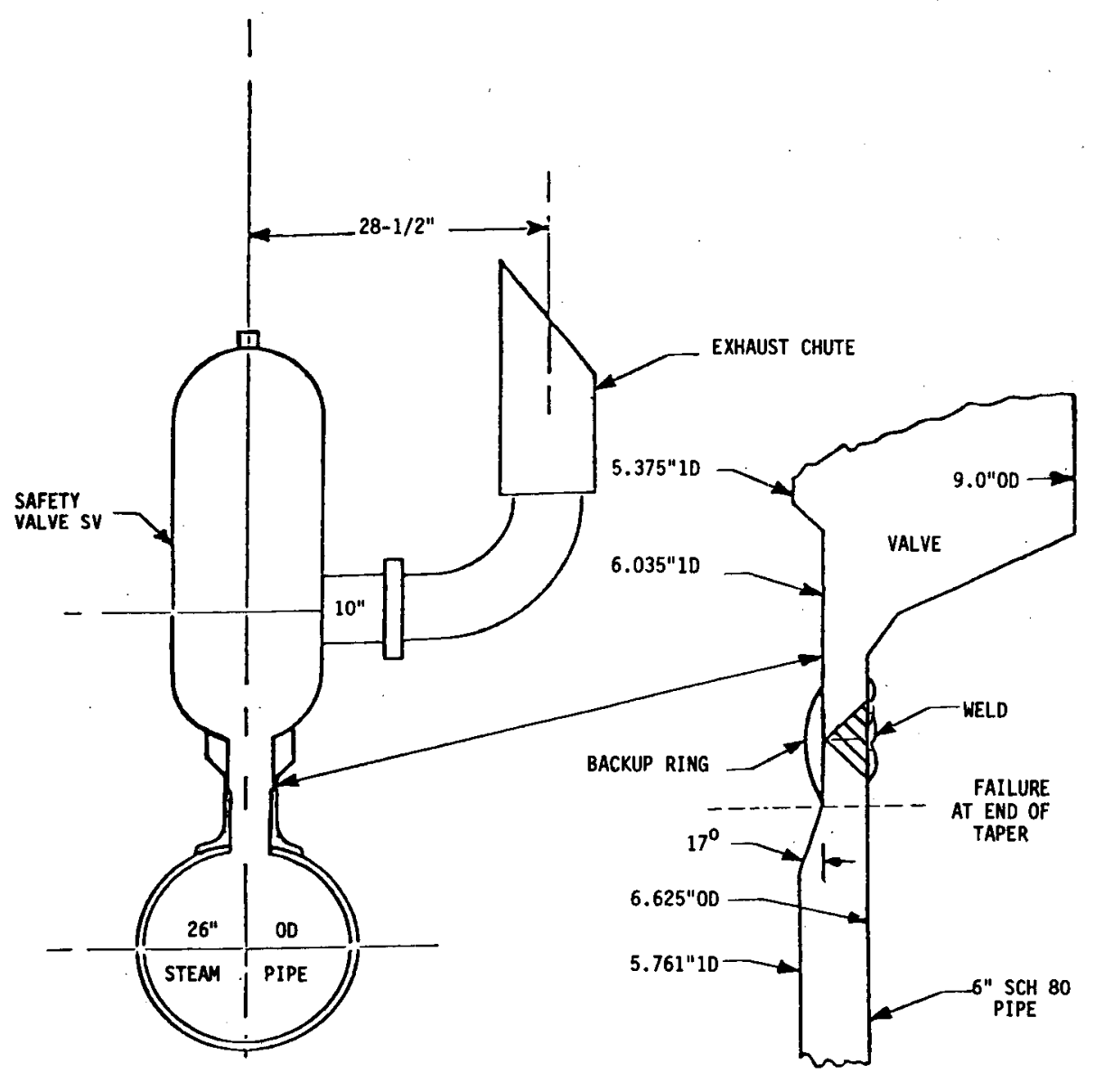

WELD JOINT

Figure L1. Schematic of safety valve connection 
Component History: Prior to the incident the weld and the pipe had undergone a satisfactory "cold hydro" test at 1356 psi. Subsequently for a period of nine days before the incident the secondary side had been operated in the pressure and temperature ranges of 800-1000 psi, $520-540^{\circ} \mathrm{F}$, respectively. Post incident inspection of the eight tested valves in the tapered zone revealed no deformation or surface marks. However, a strain of several percent is needed to break the surface scale of the hot rolled pipe. The other eight safety valves tested in steam lines $A$ and $B$ had demonstrated varying degrees of valve seat lift from a "simmer" or "weeping" to a definite "pop."

Design Code: USAS B 31.1.10

Probable Cause: All areas of the fracture surface showed plastic deformation and it was determined that the fracture was initiated by a ductile shear rupture. Since no significant material deficiences were found with respect to chemical analysis, micro structure, pre-existing defects or mechanical: properties coupled with the fracture appearance indicates that the fracture was caused by overloading. This caused stresses greater than the ultimate stress. However, analys is was unable to account for the overload.

A seemingly reasonable overload condition might be postulated by assuming the valve "popped" and the sudden rush of steam up the exhaust pipe exerted a reaction force producing a moment about the valve axis. This explanation is reinforced by the fact that the fracture initiated at a quadrant diametrically opposite to the exhaust pipe. However, there is no conclusive evidence that the valve actually "popped": since there was fresh grease on the disc which showed no trace of having been exposed to hot steam. 
L.4.2 Plant: Turkey Point Station, Unit 3 (Florida Power and Light Co.) Date of Occurrence: December 2, 1971

Plant Status: There was no fuel in the reactor. The plant was uridergoing hot functional tests and the thermal hydraulic condition was stable, with the secondary system pressure maintained at 990 psig. The main steam line isolation valves and bypass valves and other branch line isolation valves were closed. The $3 A$ steam line was completely isolated; atmospheric steam dump valves in main steam $l$ ines $3 B$ and $3 C$ were controlling in the automatic mode. Feedwater was being added to the $3 \mathrm{C}$ steam generator via auxiliary feedwater control valves from Unit 2. Concurrently a test procedure of boration of the reactor coolant was in progress when the incident occurred.

Incident Description: Three of the four safety valves in the header connected to the main steam line $3 \mathrm{~A}$ had blown off. The north header had split open. The escaping steam and the valves carried away the stile and access platforms of the safety valves and main steam isolation valves, motor operator of the auxiliary feedwater pumps, pressure transmitters. The blowdown piping for the steam generators and ductwork for the main steam lines were deformed and damaged. Though there were no personnel on the steam line platform the blast and the falling debris caused minor injuries to sixteen men.

Component History: The twelve safety valve lift pressure checks were completed about eleven days prior to the incident. After the tests were completed within a week, RV-3-1401, -1406 and -1411 were gagged due to excessive "weeping" and set pressure had to be adjusted again for RV-3-1410. 
Design Code: ASA B 31.1 (1955) and ASME B\&PV VIII

Probable Cause: Rupture of the 6 " on $12^{\prime \prime}$ weldolet due to overloading. The cause of overloading is not known except that one or more safety valves might have opened below their set pressure. No fabrication or material deficiences were found. Failure due to thermal fatigue and all types of weld related cracking was dismissed.

It is hypothesized that safety valve with lowest set pressure (1085 psi), RV-3-1400, prematurely opened producing reaction forces, which resulted in rupture at the weldolet. The header was unable to withstand this reaction load thus opening the north header. The failure of the north header triggered the failure of the valves in the south header.

L.4.3 Plant: Indian Point Station Unit No. 1 (Consolidated Edison Co. of New York)

Date of 0ccurrence: Discovered in May 20, 1972

Plant Status: The reactor was shut down within hours, after it had come on line after a two month refueling outage, to locate and plug tube leaks in steam generators No. 12 and No. 14. During the course of tube plugging operations fragments of the themal sleeve were found in the primary side of the water box.

Incident Description: The fragments were parts of themal sleeve in the 4" primary systen make-up water line where it enters the No. 12 24" primary coolant pipe. A sectional view is shown in Figure L2. The sleeve was construced of $1 / 8$ " thick type $3045 S$ rolled and longitudinaly welded into a cyclinder and circumferentially welded to a nozzle in the make-up line. The sleeve parted along both the longitudinal and circurnferential welds into four pieces. 


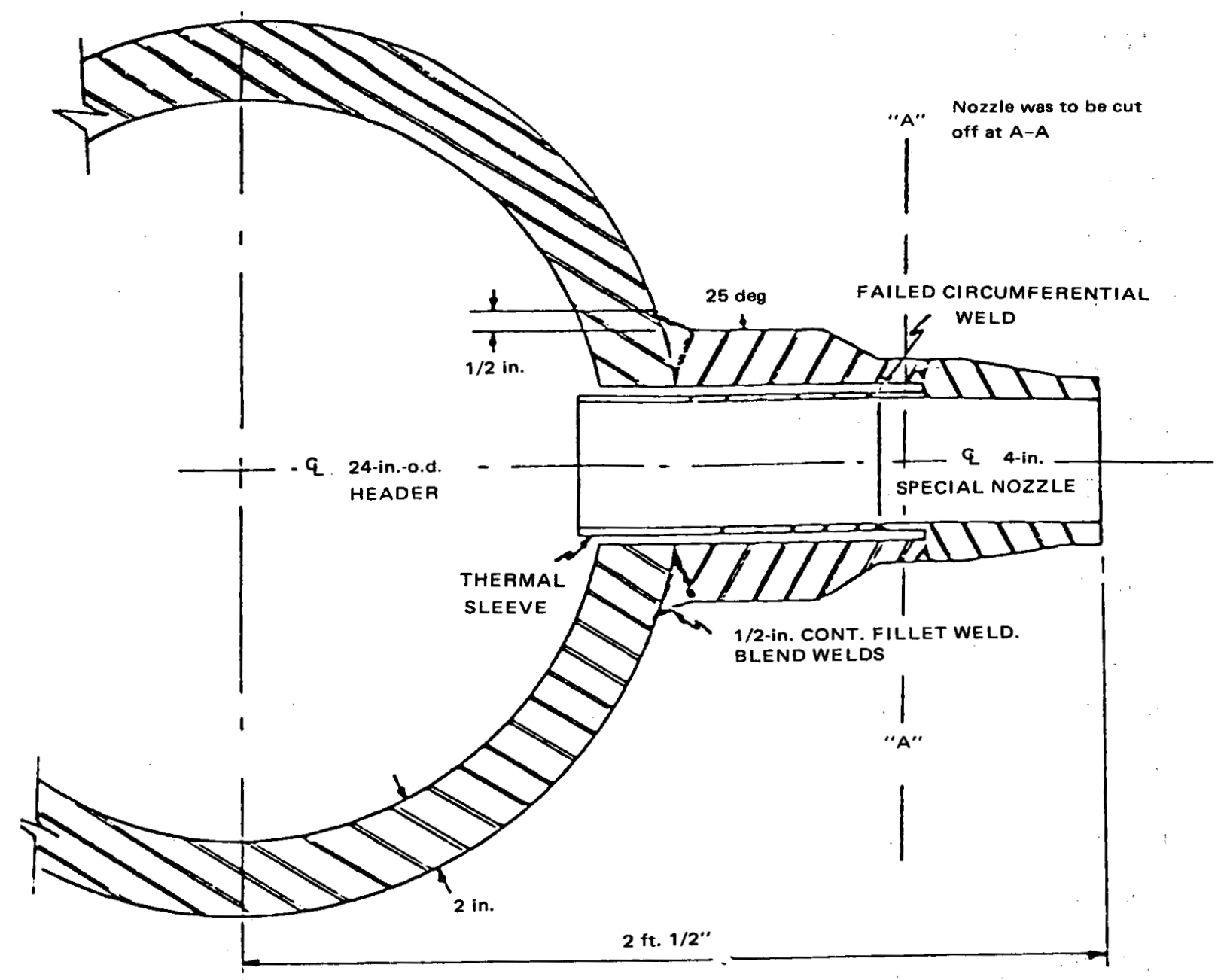

Figure L2. Schematic of original nozzle and thermal sleeve 
Liquid penetration test on the inside surface of the primary coolant pipe revealed forty cracks in either side of the pipe approximately downstream to the coolant flow.

Design Code: ASME Section III for thermal cycles and stresses Probable Cause: The mass flow difference between the letdown flow to the purification system and the make-up flow cause a variation in temperature between $120-400^{\circ} \mathrm{F}$ of the make-up water. Flow changes take place during startup, shutdown and in nornal constant load operation, albeit, less frequently in the latter case. The temperature difference between the primary coolant and the make-up water varies between $110-390^{\circ} \mathrm{F}$ and in the absence of well developed mixing causes a sharp thermal gradient within the sleeve which lead to the building up thermal stress cycles ultimately causing failure of the sleeve by fatigue.

The cracks in the primary coolant pipe resulted from the deflection of the denser cold water to the walls of the primary pipe in a wavering plume causing thermal stresses.

L.4.4 Plant: Surry Power Station Unit 1 (Virginia Electric and Power Co.) Date of Occurrence: July 27, 1972

Plant Status: The plant was shutdown for zero power physics testing and maintenance the day before the incident after it had operated at 35 percent power. Decay heat was being removed by using two condenser steam dump valves TCV-MS-107B and 108B out of four, the other two valves in bank "B", TCV-MS-105B and 106B were stuck closed. The four condenser steam valves in bank "A" were unavailable as the whole bank was isolated due to steam leakage in one valve, TCV-MS-105A. Later bank "B" was tagged out of service and the decay heat was attempted to be removed by use 
of three atmospheric steam dump valves RV-MS-101A, 101B, 101C which were inoperative. Subsequently the decay heat release valve, HCV-MS-104, was attempted to be opened by imposing a $10 \%$ demand signal when the incident occurred.

Incident Description: The decay heat release valve exhaust pipe (4"dia.) had backed out its vent sleeve (8"dia.) in the ceiling of the safeguard building, releasing steam in the upper level of the building instead of out through the vent. There was no rupture of the decay heat release valve, piping, or structural failure of its restraints. Two men died from steam burns. Component History: The decay heat release valve which is installed with its stem in the horizontal position was inspected internally after hot functional tests in February 1972 and it was determined from wear on the stem that it had been sticking in the bushing during hot functional tests. The stem was replaced and proper valve operation was demonstrated under cold shutdown condition. The decay heat release system had functioned satisfactorily on approximately twenty occassions prior to the incident.

Design Code: ANSI B 31.1 (1955)

Probable Cause: It has been estimated that there is a steady state thrust of 7800 lbs at the maximum design flow of $330,000 \mathrm{lbs} / \mathrm{hr}$ at 1085 psig and $556^{\circ} \mathrm{F}$. Such a thrust is unable to back up the discharge end of the decay heat line. For the line to disengage an estimated thrust of 8900 1bs or greater must be imposed on the pipe. It is hypothesized that the valve installed horizontally with its unsupported actuator mechanism and its past propensity of stem binding might 
have responded erratically, resulting in a sudden steam flow exceeding than that corresponding to the $10 \%$ demand signal and producing a thrust greater than 8900 lbs. The decay heat exhaust piping support system was not designed for dynamic loads imparted to the pipe under the aforementioned scenario.

\section{L.4.5 Summary of Discussed Incidents:}

From the description of accidents discussed in this section, three important observations can be made:

(i) Steam line ruptures occurred in Turkey Point 3 and H.B. Robinson 2 in the section downstream of the containment and upstream of the main steam isolation valve. Steam escaping from a rupture in this zone goes directly to the atmosphere and provides a mechanism of uncontrolled radioactive release proportional to the activity level in the secondary side assuming that the steam generator tubes are intact. However, a more safety significant scenario may be envisaged. The sudden uncontrolled release of steam causes a fast depressurization of the secondary side of steam generators (SG). The SG tubes, especially the degraded and wasted ones, are now exposed to steep increase in pressure difference. Such a condition is conducive to SG tube failures. In addition, it should be noted that the secondary side pressures were lower than the lift pressures of the safety valves in both the cases.

(ii) The above two failures and the Surry 1 incident are very similar. The valve connections were incapable of handling dynamic reaction forces imparted by the sudden opening of the valves. However, in the case of H.B. Robinson 2 there is no evidence that the valve actually "popped." 
(iii) Catastrophic pipe failures can and have occurred in a highly designed system, such as a nuclear power plant. The incorporation of such events into the operational data base is essential since they represent vulnerable areas that have, in the past, failed. If either design reevaluation or testing had not picked up the failure, the events could have occurred during operation. It is this probability that is needed before these pipe failures can be correctly utilized.

\section{L.5 Preoperation Date Application:}

If these failures are included as data points, one might conclude that the steann line break probability is of the order $10^{-2}$ events per reactor year. This is far worse than any other prediction and is of the same order of a magnitude as the worst predition based on no data.

The first objection to using these data points is that they occurred during pre-op testing and were design faults which have a high probability of detection before plant operation. This probability of detection is another factor that is difficult to calculate either by a survey of literature or data derived from experience. To illustrate, Reference 56 gives "factors of improvement" from 9 to 8100 depending on number of inspections and schedule. What is interesting in regards to these findings is that several References (56 and 49) conclude that preoperational proof test (pressure test) has a negligible effect on failure probability or conversely, pre-op proof tests have a very low efficiency or factor of improvenent.

For the failures cited here, this is obviously not true. Pre-op testing and the steps required to come up to proof (including checking relief and 
safety valve settings), has a high probability of screening out "design failures" of the type experienced at Turkey Point and H.B. Robinson. At any rate, searching for a test efficiency factor to apply to these two incidents yields the same results as before - Too wide a range of values. For the benefit of this interim report a testing factor of .01 is utilized. This factor is based solely on engineering judgment.

As a minimum, a more realistic number for a general large bore pipe rupture should be developed for use in the future risk assessment, and a specific testing efficiency should be developed for the section of piping of interest:

At the present time there is nothing to contradict a probability as high as $10^{-4}$ events per year for the sequence of a steam line break between containment and the main steam isolation valves coupled with steam generator tube rupture. This is based on using a probability of $10^{-2}$ for large bore steam line break in the section of interest usiny the data cited (worst case), and an "engineering" calculation of $10^{-2}$ for the probability of steam generator tube ruptures given a steam line break.

\section{L.6 Stean Generator Tube Rupture:}

As mentioned at the beginning of this discussion, there is enough data presently on steam generator tube rupture, to support a general conditional failure rate of $5 \times 10^{-2}$ failure per reactor year given a steam line break, see Appendix M.

The mechanism for failure is the resultant 'higher than design' pressure differential across the tube. In addition the failure rate depends on a number of factors such as the following:

a. Time since last inspection 
b. Nature of pressure transient (shock, rate of decrease, amount of decrease etc)

c. Chemistry and corrosion history of tubing

d. Integral of power level and time with prediction of errosion.

These and other factors affect both the general failure rate and the conditional probability. Additional work on the conditional probability of the steam generator tube rupture is required.

\section{L.7 Summary:}

In conclusion, this exercise has demonstrated that there exists a unacceptably wide range of probabilities predicting large bore pipe ruptures. By utilizing a literature search, data evaluation, and engineering judgment the failure rate (per year) has been determined to be:

$$
10^{-4} \leq P(P \text { ipe Rupture }) \leq 10^{-2}
$$

for zone 2 of this analysis. In the attempt to perform a realistic evaluation of the analysis of the steam line break this resulting probability range is utilized in place of the more conventional values. 


\section{APPENDIX M}

\section{ESTIMATE OF THE LIKELIHOOD OF A STEAM GENERATOR}

TUBE RUPTURE IN THE EVENT OF A MAIN STEAM LINE BREAK

\section{M.1 Probability Calculation}

Steam generator (SG) tube degradation has been a problem drawing increasing attention because of its widespread occurrence in operating PWR's and because of the potential for high costs associated with the purchase of replacement power and the repair of the affected steam generators. From a safety standpoint, two events are of concern which can result in abnormal pressure forces on possibly degraded tubing. These events are the loss-of-coolant accident, which could result in an overpressure or collapse force on the SG tubing, and the steam line break, (SLB) which could result in a burst force on the tubing. Here we are concerned with the likelihood of tube rupture as a result of a steam line break and its associated burst forces.

Degraded tubes, including leaking tubes, have been commonly observed, with tubes plugged in 25 of 68 nuclear plants covered in a 1976 survey. (57) Based on recent surveys $(57,58,59$ ) of operating plants, the major causes of SG tube degradation appear to be wastage, corrosion, stress-corrosion cracking, and denting. A change in secondary water chemistry control from highphosphate addition to all-volatile treatment has appeared to bring the wastage problem under control with a noticeable drop in failures due to this cause since 1974. Denting, which is the circumferential constriction of tubes by tube support plates, was the major cause of tube failures in 1976. 
As a result of the widespread SG tube degradation problem, inservice inspection of SG tubes using eddy current test (ECT) methods has been implemented as a means for reducing the probability of SG tube failures. NRC Regulatory Guide $1.83(60)$ describes an acceptable method for inservice inspection of PWR SG tubes. Criteria have also been developed to establish the degree of tube degradation, as identified by inservice inspection, beyond which defective tubes should be removed from service by plugging. Regulatory Guide $1.121(61)$ describes acceptable methods for establishing these criteria. A typical plugging criterion is ECT-indicated through-wall defects of 30 to 50 percent with a 40 percent defect limit indicated for most U.S. plants. (57)

In order to estimate the likelihood of a tube failure, the degree of degradation required for tube bursting must be identified.

Tests have been performed by the Pacific Northwest Laboratory (PNL) on artificially defected tubes to relate tube burst pressure to degree of wall degradation and defect length for different types of defects.(62)

At differential pressures in the range of $2400 \mathrm{psi}$ (the maximum pressure differential of interest for the SLB is closer to $2000 \mathrm{psi}$ ), tube rupture would be expected to occur if the tube degradation is in the range of 75 to 90 percent, depending on the type of defect based on these data. Section M.2 provides the PNL curve fit equations for these data and shows how this degree of degradation is determined. These limits represent minimum degrees of wall 1 degradation required for bursting which are based on values of burst pressure for defect lengths exceeding approximately an inch. For shorter defect lengths, higher degrees of wall degradation are required at this pressure differential. In calculations carried out at $\mathrm{BCL}$, based on the assumption that failure occurs when the tube attains a stress equal to the ultimate strength 
of the tube material, it was estimated that at a pressure of $2400 \mathrm{psi}$, the tube wall could be thinned to about 25 percent of its original thickness (corresponding to 75 percent wall penetration) before failure would occur.

Having determined the extent of degradation required for tube rupture, it remains to estimate the likelihood of having a steam yenerator tube with this degree of degradation which has not been plugged. Three approaches to estimating the likelihood of a tube being in a sufficiently degraded.condition to rupture in the event of a steamline break have been explored. The first is based on determining degradation rates for tubes. The second examines the uncertainties associated with eddy-current testing. The third approach uses data on the frequency with which tube leaks have been experienced, the size of the resulting leaks and assumptions about the duration of time over which a potentially large leaker was in an incipient state.

Approach 1. Deyradation Rates

There does not appear to be any direct information available on SG tube degradation rates. The fact that tube leaks have been known to occur even shortly after resumption of operation following a shutdown for inservice inspection of SG tubes might suggest that a very high degradation rate exists. Another possibility is that activities carried out in conjunction with inservice inspection, such as lancing of the buildup of sludge deposits resting on the tube sheet, may lead to additional tube leaks.

The information presented in recent SG tube failure survey articles(57, $58,59)$ does not lead to a direct estimate of degradation rate. Information is not presented on the amount of degradation observed at the various inservice inspections. The information which is presented does indicate that, 
typically at each inservice inspection, tubes continue to be identified which require plugging based on a 40-50 percent ECT-indicated wall defect plugging criterion. As indicated previously, the survey information also indicates that leaks occur even shortly after an inservice inspection and resulting tube plugging.

Development of an estimate of the likelihood of an SG tube rupture given a steam line break using SG tube degradation rates as a basis does not appear likely at this time. The data necessary to accomplish this do not appear to be available in the literature, although utilities probably have the necessary data in their records. Furthermore, any degradation rate estimate will be influenced by the uncertainties associated with the eddy current test (ECT) method, a topic which will be discussed below.

\section{Approach 2. ECT Reliability}

Steam generator tube information suggests that the ECT method may not be completely reliable in identifying the degree of tube degradation. For example, tubes have been known to leak upon resumption of operation following an inservice inspection for leaks. Leaks also continue to develop in spite of plugging at 40 percent ECT-indicated wall defects. That the ECT method is not completely reliable in identifying the degree of tube degradation is consistent with observations made in the PWR Steam Generator Tube Integrity Program being conducted at PNL. In tests conducted there on artifically defected. tubes, significant error was found in the eddy current estimates of defect depth. Eddy current signals from nearly identical flaw geometries produced very different signal patterns and flaw depth indications. For electrical discharge machining (EDM) slots, which simulate cracks in the tubing wall, 
considerable difference was found between the ECT-indicated flaw depth and the actual flaw depth. The ECT method indicated less degradation than actually existed. For uniformly thinned defect simulations, the ECT method tended to overpredict the amount of wall degradation. For artificial defects simulating elliptical wastage, the ECT method tended to underpredict the degree of tube wall degradation as the amount of actual degradation increased. Figures M1 through M3, excerpted from Reference 62, compare ECT-indicated and actual flaw depths in terms of wall thickness degraded for EDM slot defects, uniform thinning defects, and elliptical wastage defects. The data presented in these figures provide a means for estimating the actual tube wall flaw depth, or degree of wall degradation, given an indicated degree of wall degradation as determined from eddy current testing.

The PNL test data indicate that the reliability of the ECT method varies depending on the type of defect. It appears to be least reliable for those defects associated with the removal of sinall material volumes, such as cracks and pits, and most reliable for defects associated with large material volumes removed, such as uniform thinning and wastage. During inservice inspections, it does not appear possible to characterize the type of flaw identified.

Since tabulations of the PNL data presented in Figures M1 through M3 were not available to us, we have estimated the values of indicated and actual flaw depths from these figures. We then applied a linear regression to the entire data set shown on these figures to obtain both a point estimate of the actual degree of degradation and an interval estimate in which the actual degradation level is almost certain to be (a confidence level can be assigned to the interval estimate). If the interval estinate is extended on the 75 percent minimum degradation level at which tube rupture will occur at 2400 psi, then 


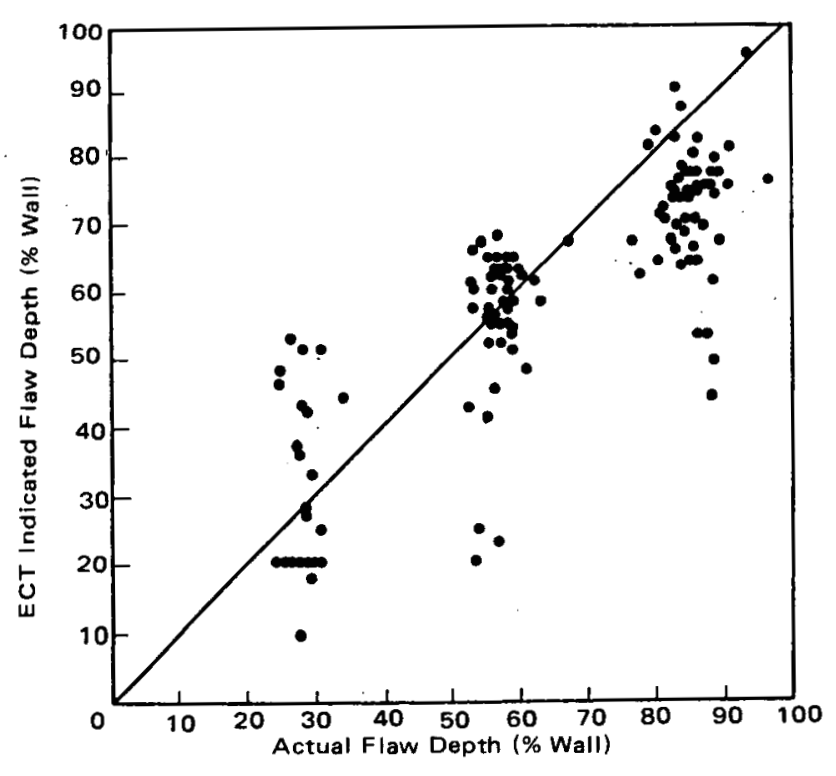

Figure M1. Comparison of ECT indicated and actual flaw depths for EDM slot defects

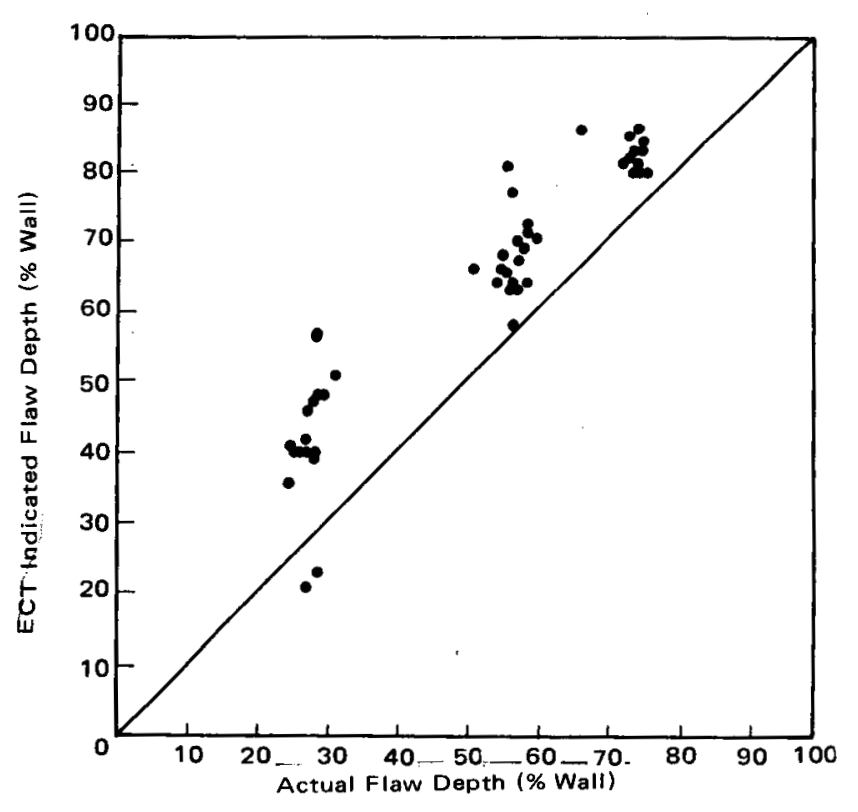

Figure M2. Comparison of ECT indicated and actual flaw depths for uniform thinning defects

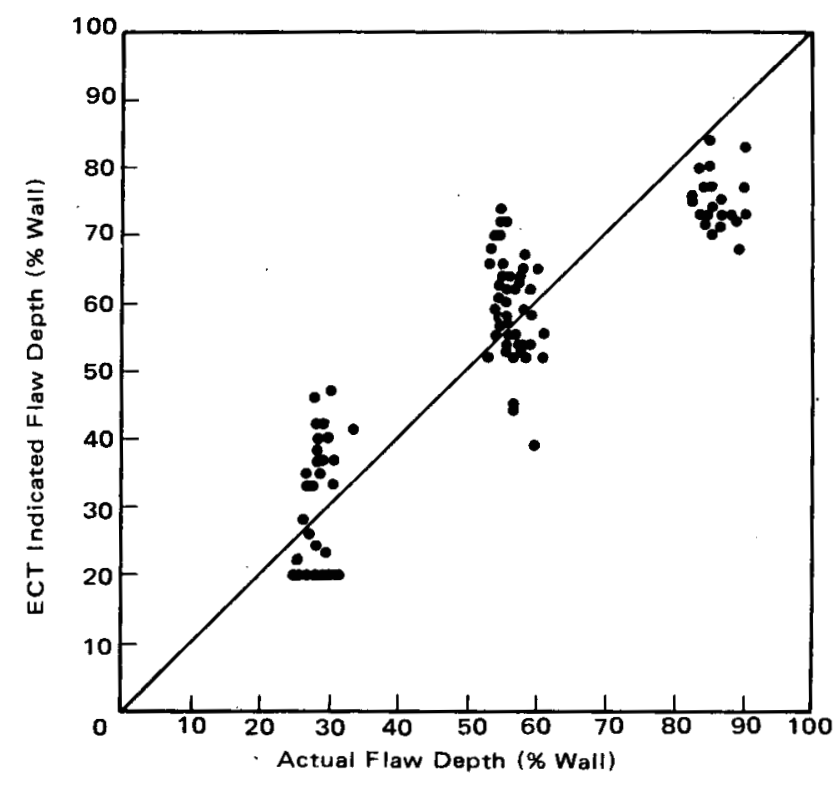

Figure M3. Comparison of ECT indicated and actual flaw depths for elliptical wastage defects 
one-half of the complement of the confidence level associated with the interval estimate can be considered to be the probability of the actual value falling above the interval.

Since SG tubes are typically plugged when ECT-indicated wall degradation exceeds about 40 percent, we looked at the interval estimates associated with indicated defect levels of 40,50, and 60 percent and confidence levels of 95 , 99, and 99.9 percent. This would result in probabilities for the estimated actual value lying outside the upper bound of the interval estimates of 2.5, 0.5 , and 0.05 percent. Table M1 sumarizes the estimated actual value, the interval estinate, and the level of confidence associated with the interval estimate.

The results presented in Table M1 present interesting information pertaining to the likelihood of exceeding an actual defect level of 75 percent, which is the minimum required for tube bursting under steam line break conditions. Typically, tubes are plugged at indicated defect levels of 40 percent or greater. If we could be certain that tubes were plugged at an indicated 40 percent defect level, the results of Table M1 suggest an extremely small likelihood that the actual defect level could be as large as 75 percent. Table M1 would suggest the probability is between 0.005 and 0.0005 . If the tubes were actually being plugged at an indicated defect level of 50 percent, then the probability that the actual defect level is as large as 75 percent is on the order of 0.025 . For plugging at an indicated defect level of 60 percent, the probability that the actual defect level is as large as 75 percent is greater than 0.1 .

Unfortunately, we do not know the actual defect level at which tubes are being plugged. All we know is that the defects are greater than 40 percent. 
TABLE M1

SUMMARY OF INTERVAL ESTIMATES FOR VARIOUS INDICATED DEFECT LEVELS AND CONFIDENCE LEVELS

\begin{tabular}{ccccc}
$\begin{array}{c}\text { Indicated } \\
\text { Defect Leve1,\% }\end{array}$ & $\begin{array}{c}\text { Best Estimate } \\
\text { Defect Level,\% }\end{array}$ & $\begin{array}{c}\text { Interval } \\
\text { Estimate,\% }\end{array}$ & $\begin{array}{c}\text { Interval } \\
\text { Estimate } \\
\text { Confidence } \\
\text { Level,\% }\end{array}$ & $\begin{array}{c}\text { Probability of } \\
\text { Being Outside the } \\
\text { Upper Bound of the } \\
\text { Interval Estimate }\end{array}$ \\
\hline 40 & 32.7 & $3.5-61.9$ & 95 & 0.025 \\
40 & 32.5 & $<0-71.1$ & 99 & 0.005 \\
40 & 32.3 & $<0-81.8$ & 99.9 & 0.0005 \\
50 & 46.8 & $17.6-76.0$ & 95 & 0.025 \\
50 & 46.7 & $8.2-85.2$ & 99 & 0.005 \\
50 & 46.6 & $<0-96.0$ & 99.9 & 0.0005 \\
60 & 60.8 & $41.8-79.8$ & 80 & 0.1 \\
60 & 60.8 & $31.6-90.0$ & 95 & 0.025 \\
60 & 60.8 & $22.3-99.3$ & 99 & 0.005 \\
60 & 60.9 & $49.3->100$ & 99.9 & 0.0005
\end{tabular}


If we assume that, at plugging, indicated defects as high as 60 percent are observed, then the number of SG tube failures that could be expected to occur if a steam line break were to occur just prior to shutdown for tube inspection would be estimated to be greater than 0.1 times the number of SG tubes being plugged per inspection at 60 percent defect. The SG tube failure survey information presented in References 1 through 3 indicated that large numbers of SG tubes are being plugged (1840 in 1974 and 1510 in 1975 in steam generators having approximately 220,000 tubes, and over 3450 in 1976 in steam generators having almost 280,000 tubes) or about one percent of the tubes are plugged per year. Using the Surry plant with its approximately 10,000 SG tubes as a basis, the numer of SG tube failures, assuming that the indicated defect level for plugged tubes is 60 percent is estimated to be greater than 10 tubes per plant if the steamline break were to occur just prior to shutdown for tube inspection. If the indicated defect levels at plugging are closer to 50 percent, the expected number of tube failures would drop to 2.5 per plant. We think that the indicated defect levels at plugging are probably below 50 percent, although data are not available to us to ascertain this. Averaged over a year, the number of tubes with greater than a 75 percent defect level would be less than just before shutdown.

The above analysis is based on the probability that the ECT method will fail to identify a crack greater than a critical value under laboratory conditions. It must be recognized that under field conditions other factors will also contribute to the failure rate of ECT to detect flaws. An important factor is human error. In performing repetitive processes such as the testing of a large number of tubes even skilled technicians will make mistakes. In addition, under field conditions cracks may occur in locations that are 
difficult to access or in which the ECT signal is masked by other features. An indicator of the fallibility of ECT is the occurrence of steam generator tube leaks during operation. Of the total numer of tubes plugged only a relatively small number were actually leaking (19 leaking tubes in 1974, 24 in 1975, and about 80 in 1976). The survey information indicates typically only one or two tube leaks occur per plant per year, although in 1976 the Surry and Turkey Point Plants (both Units 1 and 2) indicated significantly higher numbers of tube leaks.

Approach 3. Tube Leaks

If we assume that a leaking tube or an incipient leaker is likely to rupture given a steamline break, we would be forced to conclude that the probability of a tube rupture given a steamline break is high, since we know that leaks occur periodically, as indicated above, and that plants can and do operate with SG tube leaks. (Surry Technical Specifications allow operation with leaks up to $10 \mathrm{gpm}$. )

Examination of available information on leak rates associated with several SG tube leak incidents suggested that the leak openings were very small and the question arose as to whether sinall leak openings would actually lead to a tube rupture in the event of a steam line break or would only continue to leak. We examined the results of a plastic instability failure analysis of axial defects in pipes. We were interested in obtaining an estimate of the critical flaw length for a through wall flaw, as a leak would be, beyond which the analysis would indicate a rupture rather than continued leaking under steam line break differential pressure conditions. The analysis was carried out for an Inconel 600 tube with an outer diameter of 0.875 inches and a wall 
thickness of 0.05 inches. The yield and assuming annealed material at $600 \mathrm{~F}$. Figure M4 present the results of the analysis. Basically, the analysis indicates for a given flaw length and flaw depth to wall thickness ratio, the stress at which a surface flaw would develop into a through wall flaw. The analysis also indicates for a through wall flaw and a given stress level, the axial flaw length beyond which rupture rather than continued leaking is predicted. Based on this analysis, at nomal operating conditions, flaws of depth less than about 70 percent of the wall thickness would not develop into through wall flaws independent of the flaw length. Through wall flaws less than about 0.7 inches in length would leak at operating conditions. For flaw lengths above about 0.7 inches, rupture would be predicted. For the conditions of interest here, that is, for through wall flaws subjected to a stress resulting froril a stearn line break, flaws less than 0.5 inches in length would be expected to continue leaking whereas those greater than 0.5 inches in length would be expected to rupture.

Essentially no information is available on the flaw geometries for the various SG tube leaks for which we have been able to obtain leak rate information. Table M2 provides a sumnary of leak rate information we have been able to find. The methodology used in calculating the flaw area given in Table M2 is presented in Section M3.

Since the plastic instability failure analysis presented earlier requires knowledge of the flaw length to predict whether leaking will continue or rupture will occur in the event of a steam line break with an existing tube leak, we will attempt to estimate flaw lengths for the various tube leak incidents cited in Table M2. The Surry 2 tube leak of $9 / 29 / 76$ had a leak rate of $80 \mathrm{gpm}$ from a 4.25-inch long crack. Based on calculations presented in Section M3, 

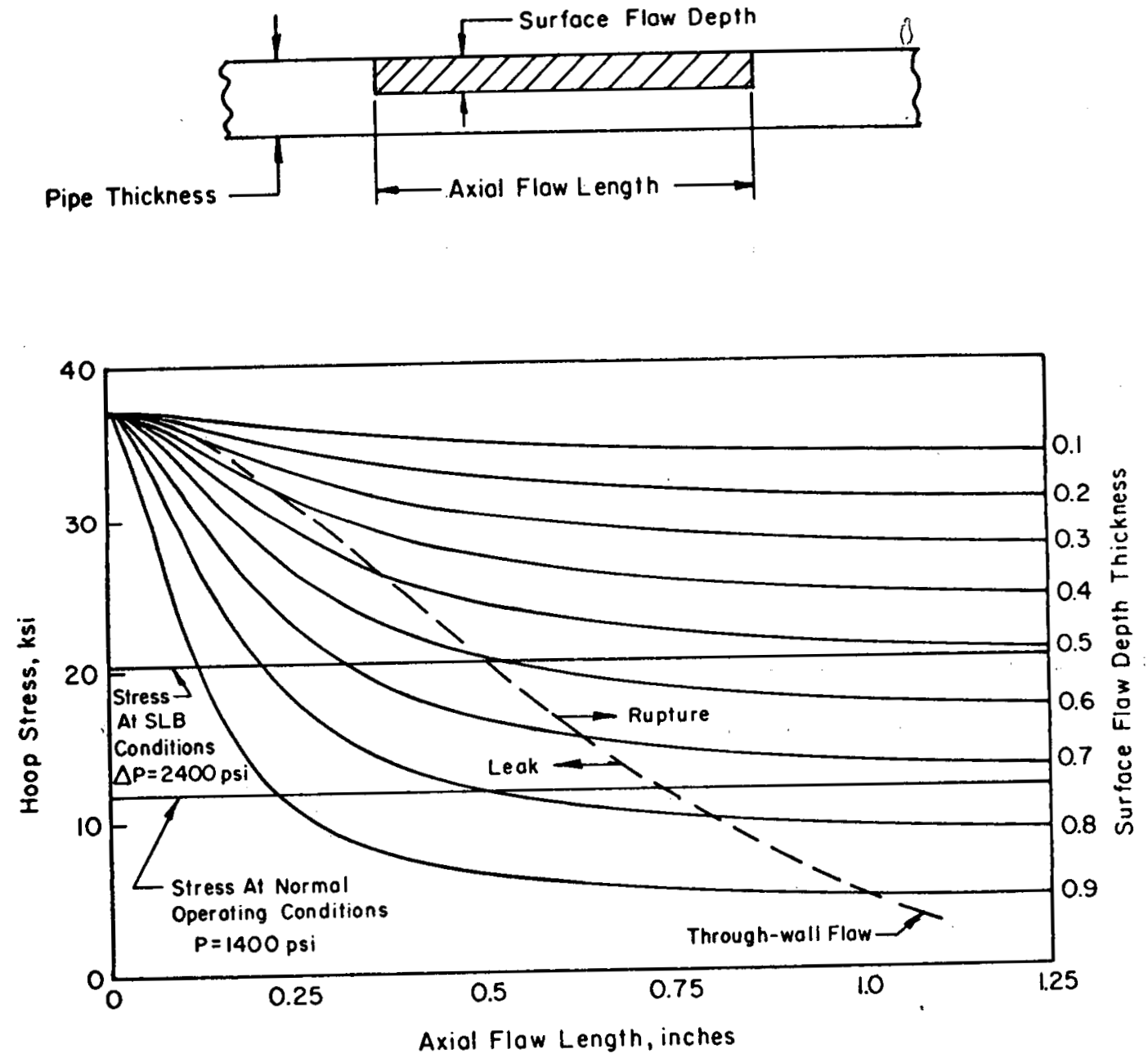

Figure M4. Hoop stress versus axial flaw length based on plastic instability failure analysis 
TABLE M2

SG TUBE LEAK RATE SUMMARY

\begin{tabular}{|c|c|c|c|c|c|}
\hline Plant & Date & $\begin{array}{c}\text { Leak Rate } \\
\text { GPM }\end{array}$ & Leak Geometry & $\begin{array}{l}\text { Calculated Flow } \\
\text { Area (in.) }\end{array}$ & $\begin{array}{c}\text { Calculated Flaw Length } \\
\text { Based on } 3 \text { mil } \\
\text { Crack Width (in.) }\end{array}$ \\
\hline Point Beach 1(1) & 1972 & 0.018 & Not Specified & $1.75 \times 10^{-5}$ & $5.8 \times 10^{-3}$ \\
\hline Shippingport $2(1)$ & -- & 0.026 & Not Specified & $2.53 \times 10^{-5}$ & $8.4 \times 10^{-3}$ \\
\hline Point Beach 2(1) & 1976 & 0.76 & Not Specified & $7.4 \times 10^{-4}$ & 0.25 \\
\hline Point Beach 1 (SGA) (2) & -- & 0.017 & Not Specified & $1.65 \times 10^{-5}$ & $5.5 \times 10^{-3}$ \\
\hline Point Beach $1(S G B)(2)$ & -- & 0.025 & Not Specified & $2.43 \times 10^{-5}$ & $8.1 \times 10^{-3}$ \\
\hline Ginna $(S G B)(3)$ & $5 / 24 / 76$ & 0.06 (Cal.) & Not Specified & $5.84 \times 10^{-5}$ & $\begin{array}{l}0.02 \\
\text { (measured) }\end{array}$ \\
\hline Oconee $3(\mathrm{SG} 3 \mathrm{~B})(3)$ & $8 / 5 / 76$ & 1.25 (Cal.) & .04 in. dia. & $1.26 \times 10^{-3}$ & --- \\
\hline San Onofre $1^{(3)}$ & $8 / 25 / 76$ & 1.5 & Not Specified & $1.45 \times 10^{-3}$ & 0.48 \\
\hline Surry $2(S G A)(4)$ & $9 / 29 / 76$ & 80 & 4.25 in. long crack & $7.78 \times 10^{-2}$ & $\begin{array}{l}4.25 \\
\text { (measured) }\end{array}$ \\
\hline Palisades (SGB) (5) & $4 / 18 / 76$ & 0.012 & Not Specified & $1.17 \times 10^{-5}$ & $3.9 \times 10^{-3}$ \\
\hline Point Beach 1 (SGB) (5) & $3 / 8 / 75$ & $125(\operatorname{Max})$. & Not Specified & 0.12 & 40 \\
\hline Surry Tech Specs $(6)$ & -- & 10 & Not Specified & $9.73 \times 10^{-3}$ & 3.24 \\
\hline ANS Standard N $237(7)$ & -- & $\begin{array}{l}0.01(100 \\
1 \mathrm{~b} / \text { day })\end{array}$ & Not Specified & $9.73 \times 10^{-6}$ & $3.2 \times 10^{-3}$ \\
\hline $\begin{array}{l}\text { (1) Nuclear Safety } 163 \text {, } \\
\text { (2) WCAP } 8215 \\
\text { (3) ORNL/NUREG/NSIC-138 } \\
\text { (4) ANS TRANS 30 11/78, } \\
\text { (5) ORNL/NUREG/NSIC-127 } \\
\text { (6) Surry Tech. Specs. } \\
\text { (7) NUREG - 0017 }\end{array}$ & $\begin{array}{l}\text {, } 1975 \\
\text { P. } 166\end{array}$ & & & & \\
\hline
\end{tabular}


the minimum crack width is estimated to be 0.018 inches or 18 mils. In his discussion of the PWR Steam Generator Tube Integrity Program results at the recent Water Reactor Safety Information Meeting (62) M. Vagins of PNL indicated that a tight stress corrosion crack was probably less than 3 mils wide. Since we are interested in estimating maximum crack lengths, we will use the 3 mil value for a typical crack width. Table I2 presents the calculated crack lengths based on a 3 inil crack width. Of the eleven tube leak incidents cited, three have calculated flaw lengths exceeding the 0.5 in. critical flaw length, as determined from the critical failure analysis carried out, beyond which rupture would be expected to occur in the event of a steam line break. It is interesting to note that the leak rate allowed by the Surry Technical Specifications would also result in a flaw length greater than the critical value predicted by the plastic instability failure analysis. If we assumed that a typical crack width were closer to the $18 \mathrm{mils}$ calculated for the Surry leak of $9 / 29 / 76$, we would still estimate that in two of the leak incidents, the calculated flaw lengths would exceed the critical flaw length for rupture. It should be pointed out that for both the Surry leak of $9 / 29 / 76$, and the Point Beach 1 leak of $3 / 8 / 75$, the plant was forced to undergo a shutdown as a result of the SG tube leak.

Of the approximately 125 total tube leaks cited in the Steam Generator Tube Failure survey articles, our analyses have therefore indicated that the flaw length in three leakers exceeded the critical length for the flaw to run. Obviously if the leak is sufficiently large at the time of penetration to force a plant shutdown it does not represent a risk as a potential rupture in the event of a steamline break. The question then becomes one of how long a preleaker is in an incipient failure condition prior to leaking in which it 
would have ruptured in the event of a steamline break. For these analyses we assume the preleaker is in an incipient failure condition for one year (one cycle) prior to penetration. Presumably the prior inspection would have picked up the defective tube if it were in an incipient failure condition. Since we anticipate 2 tube leaks per plant per year, there are at any given time 2 incipient tube leaks. Of these, $\frac{3}{125}$ would lead to rupture in the event of a steamline break. On this basis the conditional probability is estimated to be

$$
P(S G T R / S L B)=0.05
$$

\section{M.2 Determination of Defect Level Required for Tube Bursting Based on PNL} Burst Test Data

Assume the tubes are typical of those in the Surry steam generators, and that we would like to know the defect level required to burst the tubes at a pressure differential typical of that expected in a main steam line break (2400 psi). The following curve fit equations, developed from the PNL burst test data, will be used to determine defect levels required for bursting.

For EDM slots

$$
\Delta P / \Delta P_{0}=1-h / t+(h / t) e^{-0.373 L / \sqrt{R t}}
$$

For uniform thinning

$$
\Delta P / \Delta P_{0}=(1-h / t)^{1-e^{-0.13 L \sqrt{R(t-h)}}}
$$

For elliptical wastage

$$
\Delta P / \Delta P_{0}=(1-h / t)^{0.604}
$$


where

$$
\begin{aligned}
\Delta P / \Delta P_{0} & =\text { ratio of defected to undefected burst pressure } \\
R & =\text { tube inner radius, in. } \\
t & =\text { wall thickness, in. } \\
h & =\text { flaw depth, inc. } \\
L & =\text { flaw length, in. }
\end{aligned}
$$

For Surry type steam generator tubes,

$$
\begin{aligned}
& R=0.3875 \mathrm{in} . \\
& t=0.050 \mathrm{in} .
\end{aligned}
$$

Based on the PNL burst pressure data, the undefected burst pressure is about 9400 psi. Thus, $\Delta P / \Delta P_{0}$ is 0.255 , assuming that the undefected pressure is 2400 psi.

Since we are interested in the minimum defect level as given by the ratio of $(h / t)$, we can solve Equations $M .1$ and $M .2$ for $h / t$ assuming an infinite defect length or $L=\infty$. Carrying out the required calculations, the minimum defect levels, as given by $(h / t)$, required for bursting are as follows:

For EDM slots

For Uniform thinning

For Elliptical wastage

$$
h / t=0.745 \text {; }
$$$$
h / t=0.745 \text {; }
$$

$h / t=0.896$.

We can use this information to establish the degree of degradation required for tube bursting. What is still needed is an estimate of the likelihood of reaching this degree of degradation, knowing that inservice inspections are carried out regularly and tubes are plugged when they exceed a specified degradation limit, as identified by eddy current examination. 


\section{M.3 Estimation of Leak Area from Leak Flow Rate}

Two methods have been used to estimate the leak areas for the various SG tube leak flow rates cited in Table M2. In the first method, the flow rate and area are related by the following expression:

$$
Q=448.8 C_{D} A \sqrt{288 g_{C} \Delta P / \rho}
$$

where

$$
\begin{aligned}
Q & =\text { Volumetric flow rate, gpm } \\
C_{D} & =\text { Discharge coefficient, assumed to be } 0.6 \\
A & =\text { Leak area, } \mathrm{ft}^{2} \\
g_{C} & =32.2 \mathrm{ft}-1 \mathrm{bm} / 1 \mathrm{~b}_{\mathrm{f}}-\mathrm{sec}^{2} \\
\Delta P & =\text { Primary to secondary pressure differential-assumed to be } 1465 \mathrm{ps} i \\
\rho & =\text { Primary fluid density, } 451 \mathrm{~b}_{\mathrm{m}} / \mathrm{ft}^{3} \text { at } 2250 \text { psia. }
\end{aligned}
$$

Solving for the leak area $A$ in (inches) ${ }^{2}$,

$$
A=9.73 \times 10^{-4} Q
$$

In the second method, we used an average mass flux, G, based on Moody's model and the modified Burnell model. The volumetric flow rate, $Q$, is given by

$$
Q=G A / \rho
$$

where

$Q=$ Volumetric flow rate, gpm

$A=$ leak area, $f t^{2}$

$\rho=$ Primary fluid density, $451 \mathrm{~b}_{\mathrm{m}} / \mathrm{ft}$ at $2250 \mathrm{psia}$

$G=$ Mass flux, assurned to be $22,0001 b_{m} / \mathrm{ft}^{2}-\mathrm{sec}$.

$$
A=\rho Q / G
$$


Solving for the leak area $A$ in (inches) ${ }^{2}$,

$$
A=6.56 \times 10^{-4} Q
$$

In calculating the leak areas presented in Table M2, we have used Equation M.5 since it will give the larger leak area. 
APPENDIX N

SURVEY OF SOURCES OF RADIOACTIVITY IN

THE PLANT AND BARRIER ANALYSIS

\section{N.1 Introduction}

All the systems and buildings of the plant were surveyed to detemine the major repositories of radioactivity. The worst hypothetical accident scenario, which might conceivably involve each component, was considered. If the resulting release of radioactivity from a component was relatively insignificant, that component was eliminated from further consideration. A barrier analysis was performed for the remaining components to determine which additional failures would be necessary to allow the escape of radioactivity to the environment. The following sections describe the more significant components and the hypothetical release mechanisms and barriers to a release. This analysis was performed independent of the main systems/data oriented task of the main report. By reviewing the scope of the main analys is in this way, we were able to identify outlyers that were then added to the main analysis. This approach gave the risk assessment two redundant scoping proyrams to better guarantee that those sequences of importance were reviewed. Again, it must be stated that due to the number of Class 3-8 accidents possible no conpleteness statements can be made.

\section{N.2 Reactor Containment Building}

The components inside the contaiment building of Surry Power Station were ident ified from architectural drawings in the Surry FSAR: (Fig. 15.1-3, 15.1-4, 
$15.1-5,15.1-6,15.1-7,15.1-9)$. Following identification, the components were grouped on the basis of their primary system affiliation. Possibilities of release to the environment from postulated accidents due to malfunction of the components were examined to uncover any additional release mechanisms that had not already been considered. It must be noted that the components and systems within the containment are designed to meet stringent code requirements, undergo an extensive quality assurance program, and are inspected and tested according to well defined exacting schedules. The containment itself acts as a second barrier to the release of radioactivity, that might be present in the building, to the environment. For the Surry Power Station the contaiment is maintained at subatmospheric pressure. Thus, any leakage would be inwards. Gaseous and liquid waste orginating in the containnent building are sent to the Waste Processing Systerns prior to discharge to the environment. The containment can be isolated since all penetrations are protected by isolation valves.

\section{N.2.1 Reactor Coolant System (RCS)}

The principal components of the RCS are the following:

1. Three Steam Generators (SG)

2. One Pressurizer

3. One Pressurizer Relief Tank (PRT)

4. Reactor Vessel

A completeness check of liquid pathways fron the primary indicates no new accident scenario other than those analyzed in Chapter 3 of this report. The primary function of the PRT is to condense steam discharges from the three pressurizer safety valves and two power relief: valves. The PRT is partially 
filled with water at or near containment ambient temperature. The cover gas is nitrogen.

\section{Release Likelihood}

A postulated rupture of the PRT is deemed unlikely since the design pressure is twice the estimated pressure following maximum discharge of the safety valves. In addition, the PRT is protected fran overpressure by rupture discs. A rupture of these discs would discharge steam contaminated with fission product gases inside the containment. If the radioactivity level exceeds the set point value, containment isolation signal will be initiated. The PRT drains to the Primary Drain Transfer Tank Subsystem. Release likelihood from the Drain Transfer Tank will be examined in Section 4.2.3.

\section{N.2.2 Chemical and Volume Control System (CVCS)}

Nearly all the major components of the CVCS are located in the Auxiliary Building except for the following:

1. Regenerative Heat Exchanger (REHX)

2. Excess Letdown Heat Exchanger (ELHX)

The risk implications of other components of the CVCS are analyzed in the review of the Auxiliary building in Section N.3.1.

\section{Release Likelihood}

The letdown flow from the reactor coolant system passes through the shell side of the REHX where it is cooled by the charging flow in the tube side. The design pressure of the tube side is 2735 psig compared to 2485 psig in the shell side. If tube leaks are postulated then charging water would leak and mix with the letdown flow, providing no conceivable pathways of activity 
leakage to the environment. The REHX is designed to withstand themal stresses produced by step changes in temperature of the letdown flow. On detection of a faulty REHX the nomal letdown flow can be diverted through the ELHX.

The ELHX is designed to cool sufficient letdown flow necessary for injection to the reactor coolant punp labyrinth seals. The letdown stream flows through the tube side and is cooled by the component cooling water in the shell side. Since the shell side operating pressure is well below the tube side pressure, possibility of contamination of component cooling water exists if tube leaks are postulated. In this situation the temperature and activity level of the component cooling water would increase. The temperature rise of the component cooling water would be indicated in the Main Control Room and the valve connecting the component cooling surge tank to the process vent, both located in the Auxiliary Building, would be closed. Inleakage of component cooling water would cause the liquid level to rise in the component cooling surge tank and may cause spillage from the tank. The spill would eventually be collected in the Auxiliary Building sump. The ELHX can be isolated by closing valves. In addition, the values fail closed. The ELHX is also designed to accommodate temperature changes of the letdown flow.

\section{N.2.3 Primary Draiṇ Transfer Tank Subsystem (PDTTS)}

The PDTTS forms part of the Vent and Drain System and comprises of the following principal components.

1. One Primary Drain Cooler

2. One Primary Drain Transfer Tank

3. Two Primary Drain Transfer Pumps.

The PDTTS collects drain liquids originating from each primary 10op, pressurizer relief tank, leakoff liquids from valve stems and reactor coolant 
pumps. The collected liquid flows through the tube side of the primary drain cooler where it is cooled by the incoming component cooling water. The cooled liquid enters the primary drain transfer tank. An alarm is initiated if the temperature of the liquid entering the tank is high. On reaching a set liquid level in the tank the pump starts automatically, transferring the liquid outside the containment to the primary drain tank in the Boron Recovery System. One pump is on line and the other is on standby. Containment isolation valves provided in the piping penetrating the containnent are interlocked with the pump controllers. These valves open and close on pump start and stop. Containment isolation signal, when initiated, overrides the pump start signal and keeps the isolation valves closed.

\section{Release Likelihood}

The activity concentration of the liquid in the PDTTS is essentially the same as the reactor coolant activity. Since the tube side of the primary drain cooler operates at 50 psig, lower than the shell shide (100 psig), contamination of the component cooling water is eliminated in case of tube leaks. Furthermore, the primary drain cooler can be isolated by closing valves at the entry and exit points.

The capacity of the primary drain transfer tank is 600 gal and it operates at atmosphere pressure and at a temperature of $150^{\circ} \mathrm{F}$. Leakage of contents froin the tank and the piping would collect in the containment sump. Any fission product gases arising from the drain liquid which could over pressure the tank is transferred out of the containment to the auxiliary building. Here it goes to the volume control tank or through the primary drain tank vent chiller condenser, in the Boron Recovery System, to the Gaseous Waste systenl. In addition, the tank is provided with relief valve with a pressure setting of 
150 psig about 50 psig lower than the design presure of the Tank. This valve relieves to a header in the Residual Heat Removal System.

The possibility of leakage from pumps is reduced since they are canned. In addition, a faulty pump can be isolated by valves while the standby one can be brought on line.

\section{N.2.4 Residual Heat Removal System (RHRS)}

The RHRS consists of the following principal components:

1. Two Residual Heat Removal Punips (RHRP)

2. Two Residual Heat Exchangers (RHX)

The major function of the RHRS is to cool the reactor during plant shutdown and refueling operations. The RHRS comes on-line in the second phase of reactor cooldown. An additional function of the RHRS is to transfer refueling water from the Refueling Water Storage Tank (RWST) to the refueling cavity at the onset and at the end of refueling operations.

During the second phase of unit cooldown, reactor coolant enters the RHRS between the main loop outlet isolation valve and the core. The coolant flows through two motor operated gate valves in series to the suction side of two RHRPs. These two valves are interlocked with the Reactor Coolant System pressure and as such, do not open if the pressure is high. In addition there is interlocking with the RHRP/RWST isolation valve, if it is open the RHRS inlet valves renlain closed. The reactor coolant is pumped by two RHRPs through the tube side of the RHXs and returns to the cold legs of the Reactor Coolant loops via low head safety injection header. The shell side of RHX contains component cooling water. At the end of refueling operations the two RHRPs are utilized to pump water from the reactor back to the RWST. This is 
accomplished by closing the outlet line of the RHRS and opening the inlet isolation valve to RWST.

\section{Release Likelihood}

If there are tube leaks in RHX, possibility of contamination of component cooling water exists because the shell side operates at a lower pressure than the tube side. Tube leaks might actuate high radiation alam and/or show high temperature of the component cooling water. These two signals would close the control valve in the vent line from the component cooling surge tank to the process vent which are located in the Auxiliary Building. Tube or shell rupture of the RHX is an unlikely event since the design pressures are well below the operating pressures. The whole RHRS is protected from overpressurization by a relief valve at the piping connected to the discharge side of RHRPs and the pressurizer relief tank. Thus rupture of casings of the pumps, valves and piping are unlikely events. However, leakage from these components can be considered credible. In the event of a leak the reactor coolant spill would collect in the containment sump for eventual radwaste treatment.

All the components of the RHRS are located inside the containment except the lines connecting to and fran the RWST. During reactor cooldown phase the line connecting the RWST at the suction side of the RHRPs are protected by a check valve and isolation valves. Thus the possibility of backflow of the reactor coolant to the RWST is very unlikely. Similarly, breach of multiple barriers is necessary for the reactor coolant to enter the RWST in the line connecting the RWST and the discharge side of the RHRPs. 


\section{N.2.5 Neutron Shield Tank and its Cooling Subsystem}

The neutron shield tank (NST) forms part of the primary shielding. The cooling subsystem is provided to cool the water in the NST. The principal components are:

1. One neutron shield tank

2. One neutron shield tank surge tank

3. Two neutron shield tank coolers

4. One corrosion control tank

The NST is made of carbon steel and forms an annular cylinder surrounding the reactor vessel. It has a radial dimension of approximately 3 feet and is approximately 22 feet in height. From architectural drawings, the volume of the tank has been estimated to be approximately 27,000 gal. The NST contains water which is heated by neutron and yamma radiation. The heated water rises by natural convection in the tank and flows through one of the coolers where it is cooled by the Component Cooling Water Subsystem. The other cooler is on standby. The surge tank acconmodates the themal expansion of NST water. A level indicator in the surge tank is used to add water from the component Cooling Subsystem on a low level signal. The cooled water from the cooler re-enters the NST at the bottom. A corrosion inhibitor is added manually from the corrosion control tank when the reactor is not operating.

Release Likelihood

The activity inside the tank would result from activation of impurities in water and the steel tank material. The contribution from these two mechanisms would be very smal1. The amount of tritium formed from deuterium is small since it forms one part in 7,000 in natural water and the cross-section is about $6 \mathrm{mb}$ for thermal neutrons. Radioactive isotopes of nitrogen, $N^{16}$ and $N^{17}$, formed by fast neutron irradiation of $0^{16}$ and $0^{17}$ pose no real 
hazard since the amount formed is negligible and furthermore, their half lives are of the order of seconds $\left[T 1 / 2\left(N^{16}\right)=7.4\right.$ secs, $T 1 / 2\left(N^{17}\right)=4.1$ secs]. The amount of $\mathrm{C}^{14}$ formed from $0^{17}(n, \alpha) c^{14}$ is estimated to be approximately $10^{-3}[\mathrm{Ci} / \mathrm{yr}]$ assuming a thermal neutron flux of $10^{9} \# \mathrm{n}^{\prime} \mathrm{s} /$ $\mathrm{cm}^{2}-\mathrm{sec}$. and the weight of water in the tank, approximately $1.05(5) \mathrm{kg}$. From the above analysis it can be inferred that the tank has a very small inventory of activity. However, even if tank leak is postulated.all the liquid would collect in the containment sump. Gases formed by radiolys is of water which could conceivably overpressure the system collect in the gas space in the surge tank and are processed by the Vent and Drain System. Any likelihood of contamination of component cooling water by the NST water in the cooler as a result of leaking tubes is averted because the shell side operates at 3.3 higher pressure than the tube side.

\section{N.3 Auxiliary Building}

The auxiliary building is the most complex inventory volume in the plant, containing hundreds of components divided anongst various systems. In order to insure completeness, a list of equipment and building features such as sumps, drains, and vents was generated. The list was divided into major system categories as follows:

.1 Chemical and volume control system

.2 Boron recovery system

.3 Contaminated waste handling systems including vents and drains.

Equipment such as the steam blowdown tanks which does not fit in major categories were assigned on the basis of proximity to major system components. In all cases, flow paths into and out of the Auxiliary building were traced 
using the FSAR drawings, to insure consideration of all possible sources of radioactivity within the Auxiliary building.

In the present work it was not possible to calculate radioactivity levels in all components or pieces of equipinent. Appendices $C, D$, and $E$ present results for certain important components. The FSAR gives technical specification allowances based on one percent failed fuel ratio for a few selected components. So, limited conclusions can be drawn based on the relative magnitudes of the source terms.

\section{Liquid Releases}

All components containing contaminated liquid (including evaporator bottans fluid) are located below grade and within radiation shields (concrete walls). A spill from any single component or group of components such as the Boron Recovery evaporators, would be contained within the building and routed to the contaminated waste handing system. This would result in possible over-exposure to personnel in the immediate vicinity (within the concrete enclosure) of a spil1, but no release of liquid out of plant."

The Boron Recovery System hold-up tanks and test tanks, provide liquid paths out of the building. These are discussed under the Boron Recovery System. Other paths are the Steam Generator Blowdown tank drain discussed under CVCS, and the Auxiliary Steam Drain Receiver.

\section{Gaseous Releases.}

While it was not possible in the time frame of this report to calculate actual activity levels in all of the gaseous volumes and traps within the Auxiliary building, there was no potential source greater than the waste gas decay tanks which are specifically treated in Section 2.5. The Auxiliary 
building ventillation exhaust is monitored and can be bypassed through charcoal filter banks (from control room) if such a release is experienced. The time required for reaction and switching is not given.

\section{N.3.1 Chemical and Volume Control System (CVCS)}

The functions of the CVCS are described in Appendix D and Figure D2 which is a schematic of the system. During normal operation, reactor coolant from the discharge side of the reactor coolant pump in loop 1 enters the letdown line. The purification flow goes through the shell side of the regenerative heat exchanger where it is cooled. This is followed by a pressure reduction at the letdown orifice. After the first stage of temperature and pressure reduction the purification flow leaves the containment building and enters the auxiliary building. Here the flow passes through the tube side of the nonregenerative heat exchanger for further temperature reduction. This is followed by a second stage of pressure reduction at the low pressure letdown valve. From there it passes through the letdown filter to the mixed bed demineralizers where anionic and cationic impurities are removed. The cation bed demineralizer downstream of the mixed bed demineralizer is used about 10 percent of the time to control the cesium and lithium activity. After its passage through the demineralizers it passes through the reactor coolant filter and enters the volume control tank via a three-way modulating valve. From the volume control tank the purification flow goes to the charging pumps where the pressure is increased above the primary system pressure. Then the flow leaves the auxiliary building and enters the containment building where is passes through the tube side of the regenerative heat exchanger. The purification flow re-enters the primary system at the discharge side of the reactor coolant pump in loop 2 . 
A portion of the charging flow goes through seal water injection filters located in the auxiliary building and passes to the containment where it enters the reactor coolant pump providing shaft seal water. Part of this flow re-enters the auxiliary building and goes back to the discharge side of the volume control tank through a seal water filter and seal water heat exchanger. The following are the principal components of the CVCS for one unit of Surry in the Auxiliary Building:

1. Volume Control Tank

2. Mixed Bed and Cation Bed Demineralizers

3. Non Regenerative Heat Exchanger

4. Seal Water Heat Exchanger

5. Letdown Filter

6. Reactor Coolant Filter

7. Seal Water Filter

8. Charging Pumps

\section{N.3.1.1 Volume Control Tank}

After passing through mixed bed demineralizer and the reactor coolant filter, the letdown flow enters the VCT through a spray nozzle located inside the tank. The vapor space in the tank contains primarily hydrogen and water vapor. The activity in the volume control tank is estimated in Appendix D.

Release Likelihood from the VCT Rupture Event

If the level of liquid in the VCT rises above the operating set point the cover gas pressure would rise. Two redundant channels monitor the level of liquid in the VCT. If the level rises a signal from one channel is sent to a 
proportional controller aligned with a three-way valve located upstream of the VCT and downstream of the Reactor Coolant Filter (Surry FSAR Figure 9.1-2). This valve splits the letdown flow accordingly, a part to the Boron Recovery System, and a part to the VCT. This operation is also followed in the 'dilution' mode. If one channel fails then the back-up channel comes on line automatically to divert the flow and initiates a 'high level' alarm in the Main Control Room. In addition, the VCT is also provided with a. relief valve. This valve relieves to the Boron Recovery system from whence it goes to the Gaseous Waste Processing System.

The two VCTs are protected from the rest of the Auxiliary Building by concrete walls about 3 feet thick and the tanks are isolated fron each other by a concrete wall (Surry FSAR Figure 15.1-12). Thus the possibility of a tank damaye from impact by missiles or monorail related accidents can be ruled out. The possible common mode rupture event of one tank produced by shock waves generated from a postulated rupture of the other tank is improbable since the tanks are isolated from each other.

In actuality, if the postulated rupture occurred, all of the liquid would be contained within the auxiliary building and eventually find its way, via sumps and drain lines, to the liquid waste system. The radioiodine gases released would at first pass out through Auxiliary Building ventilation system, and after initiation of high radiation alarn, would be diverted through charcoal filters before release to the atmosphere.

\subsubsection{Mixed Bed and Cation Bed Demineralizers}

There are two mixed bed and one cation bed demineralizer in the CVCS for each unit of Surry. One of the mixed bed demineralizer is on standby status. 
Under nomal operating conditions the cation bed demineralizer operates about ten percent of the time. The demineralizers may contain a rather large inventory of radioactivity. The solid waste, consisting of filter sludge, resins and evaporator bottoms is, an average for PWR's, $670 \mathrm{Ci} / \mathrm{yr}$. (63) The major isotopes are C058, C060, Cs134, and Cs137. According to the surry FSAR, resins are replaced when the decontamination factor falls below a certain value or the external dose rate exceeds a setpoint. In addition, the resins in a demineralizer will contain comparable amounts of $I$ isotopes and other short-lived isotopes.

Release Likelihood

The demineralizers are positioned in concrete vaults below grade with concrete plugs over the vaults in the Auxiliary Building (Surry FSAR Figure 15.1-11). So, even if rupture of the demineralizer tank is postulated the contents would still be contained within the vault. In addition an energy source, i.e. an explosion of sorts, is needed to cause dispersal of the postulated spillage. The operating conditions of the demineralizers in comparison to the design specifications makes the rupture event very unlikely.

The operation of transferring spent resins to the spent resin catch tank in the decontamination facility building was investigated for possible pathways to the environment. Water from the primary water storage tank (located outside the Auxiliary Building) is added to the spent resins to create a slurry before it enters the spent resin tank (Surry FSAR Figure 11.2.3-3). The inlet of primary water is through pumps and a number of check valves (Surry FSAR Figures $11 \cdot 2 \cdot 3-3$ and 9.2-3). Hence the contamination of the primary water storage tank water from backflow of the slurry is improbable. 
A postulated break in the pipe and other components involved in flushing of spent resins would spill the liquid in the Auxiliary Building sump to be collected by the Vent and Drain System for subsequent processing. Thus the risk implications to the general public from the postulated spill is inconsequential. Completeness review of the spent resin catch tank is performed in Section 4.6 .

\section{N.3.1.3 Other Components in the CVCS}

The letdown, reactor coolant, seal water, and seal water injection filters have disposable synthetic filter elements. The filter vessels are made of austenitic stainless steel and have connections for venting and draining. Thus, failure due to corrosion or rupture from overpressurization of the filter vessel is averted. The filter vessels can be isolated by means of closing valves at the inlet and at the outlet. A bypass line with a valve (nomally closed) is provided in parallel across each filter vessel. If leaks devel op in the filter vessels they can be isolated and the flow diverted through the bypass line. Thus, the amount of leakage, if it occurs, can be kept to a minimum and the process continued through the bypass line. The risk implications from the postulated leakage is inconsequential to the general public. However, occupational exposures might result during the operation of replacing spent filter elements since they contain entrapped radioactive particulates.

The nonregenerative heat exchanger (NRHX) cools the purification flow to the operating temperature of the demineralizer located downstream of the NRHX. The reactor coolant flows through the tube side while component cooling water flows through the shell side. The design pressure of the tube and shell side 
of the NRHX are 600 psiy and 150 psig respectively. Although operating pressures are not precisely known the tube side is maintained at a higher pressure than the shell side. If tube leaks are postulated contamination of component cooling water (CCW) would occur. The consequences of this possible contamination will be essentially the same as in the Excess letdown heat exchanger which has been analyzed earlier in Section 4.2.2.

The Seal Water Heat Exchanger (SWHX) cools the reactor coolant from the reactor pump seals, excess letdown heat exchanger and the bypass flow originating from the charging pumps before entering the discharge line of the volume control tank. Sirilar to the NRHX the reactor coolant flows through the tube side and CCW in the shell side. The design pressure of the shell and tube side of the SWHX are the same, 150 psig. Although operating pressures are not known infomation from Surry FSAR Section 9.1.2.5 indicates that the operating pressures of the tube and shell side are nearly the same. Thus, if tube leaks are postulated leakage could occur in either direction. However, the tubes are welded to the tube sheet to reduce leakage. A bypass line with a valve (nomally closed) is provided in parallel to the SWHX so that it can be isolated, and with the bypass line normally closed valve open the fluid has a pathway to the discharge line of the volume control tank. As in this previous case of the NRHX possibility of contamination of CCW exists. The train of events which follow tube leaks and the consequences thereof are similar to that described in Section N.2.2 for the Excess Letdown Heat Exchanger. Surfaces in contact with the reactor coolant in both these heat exchangers, NRHX and SWHX, are made of austenitic stainless steel thereby reducing tube failures due to corrosion. 
Three single speed horizontal centrifugal charging pumps operate on two distinct modes: 1 . charging of reactor coolant and, 2. safety injection. To minimize leakage of reactor coolant to the Auxiliary Building atmosphere the pumps are provided with a mechanical seal and auxiliary gland bushing, and a leak off connection between them. The leakoff is transferred to the primary drain header. Each pump is provided with two seal vents and two casing vents to reduce pressure buildup. The pumps are protected from blocked discharge during testing and too low charging flow by maintaining a bypass recirculation. Charging pumps can be isolated by closing motor operated valves located at the suction and the discharge side. Activity measurements (EPRI 274-1) in exhaust air from the ventilation of the charging pump roams of three plants during power operation accounted for 6 to 15 percent of $I-131$, and 6 to 38 percent of tritium of the total I-131 and tritium releases from the Auxiliary Building. Following hot shutdown conditions releases increased to 7 to 18 percent of I-131, and 4 to 54 percent of tritium. Most of the $I-131$ release is in the organic form. The increase of I-13I release may be due to 'iodine spiking' occurring in the primary coolant following shutdown.

\section{N.3.2 Boron Recovery System}

The Boron Recovery Systern (BRS) is sized for, and common to, both units one and two at the Surry plant. .The BRS. receives primary coolant let-down from the CVCS system via piping in the drain system. It provide for the degasification (using the gas-stripper) and storage of the borated radioactive water (outside of the Auxiliary building) in three 120,000 gal. Boron Recovery Tanks. These tanks and the system are sized to accept let-down flow from two cold shutdowns froin full power and one cold shutdowns from full power from the 
second unit in seven days with a boron concentration equal to $100 \mathrm{ppm}$ in the first unit and one month out of phase in the second unit. The Boron Recovery Tanks are assumed to be 10 percent full at the beginning and the Boron Evaporators are 75 percent available during the 7 days. The gas stripper is capable of removing gases from both units at maximum let-down flow.

The de-gased, borated water is fed back to the Auxiliary building to the evaporators where distillate and boric acid is produced. The distillate is again directed out of the Auxiliary building to the Test Tanks (2 of 30,000 gal, ea). Here the water is sampled and if acceptable, it is routed back to the Auxiliary building for a final stage of demineralization, through the Boron Clean-up Ion Exchangers, and then out again to the Primary Water Storage Tanks. The concentrated boric acid solution is collected in the Evaporator Bottoms Tank, and after sampling, is either routed to the CVCS (Boric Acid Tanks) for re-use, or the Liquid Waste Disposal System for disposal.

The major components in the BRS are listed in Table N1 . Other components, including pumps, valves, and heat exchangers were not treated as sources of radioactivity in this survey, although they are considered as possible points of systern failure.

\section{N.3.2.1 Release Likelihood for BRS Components within the Auxiliary Building}

There is no fluid path in the Boron Recovery System leading directly to the atmosphere. Liquid is taken from the CVCS system and distillate is directed back to the CVCS system. All gases are vented or driven to the waste yas system. Contaminated liquid wastes such as high radioactivity evaporator bottoms are directed to the liquid waste handling system. A rupture of any component in the system involving liquid spill will be contained within the 
TABLE N1

BORON RECOVERY SYSTEM MAJOR COMPONENTS

Inside the Auxiliary Building

1. Primary Drain Tank

2. Gas Stripper

3. Gas Stripper Surge Tank

4. Cesium Removal Ion Exchanger

5. Boron Evaporator

6. Distillate Accumulator

7. Evaporator Bottoms Tank

8. Boron Clean-up Ion Exchangers

Outside the Auxiliary Building

1. Boron Recovery Tanks

2. Test Tanks 
Auxiliary building and eventually, via sumps and drains, end up in the liquid waste treatment system. Gaseous releases are possible via the Auxiliary building ventilation system in event of rupture. The worst case would be the rupture of the gas surge tank which can contain as much as 500 SCFM of contaminated gases. Most of this volume consists of nitrogen, oxygen and hydrogen. Even if it were completely full of noble gases with the same proportion of I-131 as in the gas decay tanks, the maximum release would be below that of the gaseous decay tanks which is treated in detail in Chapter 2 of this report. There is also an additional barrier not present in the case of the gas decay tanks, the Auxiliary building ventilation system monitors and filters. Upon detecting high activity, this gas release (mixed with Auxiliary building air) would be exhausted through the Auxiliary building filters. Therefore, catastrophic rupture of any component of the Boron Recovery System, within the Auxiliary building would not result in any significant exposure to personnel except anyone caught within the concrete shielding of the component at the time of the incident.

\section{N.3.2.2 Release Likelihood for BRS Components Outside of the Auxiliary Building}

The Boron Recovery System is connected to two sets of tanks located outside the building. The first set is the Boron Recovery Tanks ( 3 of 120,000 gal capacity each). They nomally contain primary coolant which has been processed through the gas stripper and the cesium removal ion-exchanger. The line feeding thess tanks are one 4" diameter with a nomal flow rate of 240 gpm. The tanks are not pressurized, and vents lead to the gaseous waste system. The line feeding the evaporators is 2 " in diameter with a flow of 150 gpm (pumps located near tanks). The tanks are equipped with level sensors, 
and are individually diked so that any leak or rupture can be collected and transferred to one of the other tanks as volume becones available. Line ruptures can be detected by level changes, and small line leaks should be detectable by inspection of the line trenches. If these lines are not run in trenches, they should be examined periodically for leakage (more frequently than the ASME code requirements of once every 10 years). These tanks are detailed because there is one possible sequence that could lead to a significant release.

The gas-stripper can be bypassed in case of malfunction or inoperability, and therefore the Boron Recovery tanks can be full of liquid with as high a concentration of noble gases as is produced in the primary coolant. If a tank were to rupture at this time, there would be a release of noble gases directly to the atmosphere. The magnitude of this release would depend on the following factors:

.1 Original concentration

.2 Atmospheric temperature

. 3 Location of tank rupture (tanks are vented to gaseous waste removal system)

.4 Fluid temperature in tank.

Since the probability of a tank rupture occurring under these circumstances is of the order of $10^{-6}$ events per tank year, the actual potential release has not been calculated. A combination of the worst possible factors could result in a release in order of maynitude equal to that of the gas decay tanks (See Appendix C).

The second set of tanks is the Boron test tanks (2 of 30,000 gal. each), located at grade level on the outside of the Auxiliary building along its west wall. They contain primary grade coolant that has been stripped of gases, 
filtered and processed by the evaporators. These tanks, both because of their size and location in the Boron Recovery Cycle, present a negligable risk compared to the Recovery tanks. They would not contain significant amounts of noble gases since they can only be filled from the boron distillate accumulators.

\section{N.3.3 Liquid Disposal System}

The liquid waste disposal system processes liquid radioactive wastes received from a variety of sources. The basic processes used to remove and control radioactive effluents are filtration, to remove particulate matter; evaporation, to concentrate radioactive constituents into smaller liquid volume and to separate liquid and gaseous phases; demineralization, to remove dissolved material; natural decay of radioactive isotopes, by holdup; and dilution, to reduce the concentration of radioactive liquids. A detailed discussion of the liquid waste system and an estimate of the inventories in various tanks is given in Appendix E.

\section{Release Likelihood}

Analys is of the Surry liquid waste disposal systern indicates that requirements for inadvertently releasing liquid waste are either due to a leak or rupture of a component in the liquid waste system, or the human error of incorectly valving the waste to another system. Also, there are large differences in radioactive concentrations, depending on where a component may be located in the system. The components associated with transfer of the evaporator bottoms will contain the largest concentrations of radioactivity. The next largest concentrations of radioactivity would be contained in the 
components in the system prior to the evaporator $\left(<10^{-1} \mu \mathrm{Ci} / \mathrm{Cc}\right)$. Components in the system following the evaporator see distillate which should have concentrations at least three orders of magnitude less than components prior to the evaporator. It is noted that leak or rupture of any of these components would result in radioactive liquid of some concentration flowing to an auxiliary building sump, and then being transferred to the vent and drain system. Liquid waste in the vent and drain systen, however, flows back to the liquid waste system for processing if the radioactive level exceeds a predetermined maximum. Therefore, in order to release concentrated radioactivity to the environment it would be necessary to postulate a fault in the vent and drain system, in addition to the fault in the liquid waste system.

To examine the consequences of human error resulting in an inadvertent transfer of liquid waste to other systems, the diagram of the piping and associated components for transfer of evaporator bottoms to the drumming station was analyzed. Two nomally closed valves, designated $V 1$ and $V 2$, were identified that, if opened inadvertently, would cause release of evaporator bottom effluent to other systems. No valves or components were identified that could result in direct evaporator bottom release to the environment. If VI were inadvertently opened, evaporator bottoms would be transferred to the vent and drain system. However, this transfer occurs in an area of the vent and drain system that would result in the effluent being recycled to the liquid waste systen. Thus, this accident sequence requires a fault in the vent and drain systen concurrently with the inadvertent valve opening. A similar situation holds for the inadvertent opening of sample valve $V 2$. The evaporator bottoms effluent would flow into a saniple sink and then to the vent and drain system, where the effluent would be recycled to the liquid waste system. The 
conclusion is, in order for evaporator bottoms to be released to the environment, at least two faults must occur, one in the liquid waste system to reroute the effluent; and at least one in the vent and drain system, to release the effluent to the environment. No valves were found which, if left in the wrong position, could drain unprocessed effluent directly to the environment.

The conclusion is that the protection offered by the closed loop paths between the liquid waste system and vent and drain system require at least a double system failure to result in release of radioactivity to the environment.

\section{N.3.4 Gaseous Waste Processing System}

During normal operation, gaseous fission products are stripped from the reactor coolant. In addition, radioactive gases are evolved during leaks within the containment, when purging or venting vessels containing reactor coolant and when taking samples. These radioactive effluents are processed in the gas waste processing system. High level gaseous wastes may be stored in order to allow for decay before they are released. The gas waste system is described in detail in Chapter 2. In order to complement the earlier discussion of the gas waste processing system, in this chapter we will discuss the recombiner package.

The gases which are stripped from the reactor coolant in the gas stripper consist primarily of hydrogen, nitrogen and fission gases. The purpose of the recombiner package is to remove the hydrogen, so that the volume of gas which must be stored in the decay tanks is reduced. The recombiner system is designed according to Section III-C of the ASME Code. The incoming gas stream, which contains hydrogen, is mixed with the required amount of oxygen and 
nitrogen so that the resultant hydrogen concentration is 1 ess than $3 \%$. This is below the lower hydrogen flammability limit of $4.4 \%$. Hydrogen analyzers on the recombiner feed stream shut two valves if the hydrogen concentration exceeds $4 \%$. Simulataneously nitrogen purge lines open to dilute the gas stream. The mixture is pumped to a preheater, thence to the recombiner. Ninety-nine percent of the hydrogen and oxygen is catalytically reacted to produce water vapor. The effluent gas from the recombiner is cooled in the after cooler, and then flows to the moisture separator. The water vapor condenses and is drained to the liquid waste disposal system. Oxygen analyzers on the effluent gas stream reset the oxygen addition controller disposal system. The gas stream is recycled through the recombiner or may be bled of $f$ to the waste gas surge tank. The bleed stream is regulated by a pressure control valve. The hydrogen analyzers on the effluent stream shut the valve in the event of a high hydrogen concentration.

Release Likelihood

The hydrogen concentration in the gas stream is maintained bel ow the flammability level. There are two redundant hydrogen analyzers on the influent stream, so there is a low probability of both analyzers failing low and causing the flammability level to be exceeded. The system is protected against overpressure by a relief valve which vents to the process vent upstream of the filters and process vent blowers. The recombiner is connected to the liquid processing system via the drain in the moisture separator. The conceivable mechanisms by which a release could occur from the recombiner would be due to a leak in some component or a failure through these valved connections. 


\section{N.3.5 Miscellaneous Components}

\section{N.3.5.1 Auxiliary Steam Drain Receiver}

There are no flows or volumes given in the FSAR, and this component would not contain any radioactivity except in case of heat exchanger leakage. Since it is vented to atmosphere, the vent should be monitored so that leakage can be detected.

\section{N.3.5.2 Steam Generator Blowdown Tank}

The Steam Generator Blowdown Subsystem (SGBS) forms part of the Secondary Vent and Drain System of Surry. The SGBS is identical but separate for Units 1 and 2. The steam generator (SG) blowdown may become contaminated due to primary-to-secondary leakage and the flashed fraction of the blowdown i.s discharged directly to the atmosphere without any intervening purification. The blowdown rate for each $S G$ is controlled by a manually operated needletype flow control valve. Blowdown lines, 1" diameter, steming from each SG penetrates the containment and passes into the Auxiliary Building to a header. The blowdown passes from the header into the blowdown tank. A part of the blowdown flashes into steam and is vented directly to the atmosphere through a 18" diameter vent pipe situated at the roof of the Auxiliary Building. The condensate in the tank is normally gravity drained to the circulating water discharge tunnel.

There are two radiation monitoring channels (gamma scintillation detectors) per unit, and one of them continuously measure the activity level of the liquid phase of the SGs, of a composite blowdown sample, taken from a point ahead of each flow control valve. If the activity level is lower than the set point, $3.5 \times 10^{-3} \mu \mathrm{Ci} . \mathrm{Cc}^{-1}$, of the radiation monitor, blowdown is allowed. 
If the contamination exceeds the set point an alarm is initiated in the Main Control Room. Then, the operator shuts off all blowdown from the affected unit and diverts the liquid in the blowdown tank to the Vent and Drain System. In this situation, a valving arrangement permits sampling of each SG liquid to detemine which $S G$ is leaking by one of the radiation monitors. The other detector measures the activity of the combined blowdown from the other two steam generators. There is another radiation monitor on the blowdown tank vent pipe which measures the vapor phase activity of blowdown escaping to the atmosphere. Information relating to the set point of this monitor and its control function is not available from the Surry FSAR. Loss of power does not interfere with radiation monitoring, $S G$ blowdown and alarm initiation. Trip valves on each side of the containment wall on all three of the blowdown lines close on a containment isolation signal.

\section{Release Likelihood}

The maximum amount of radioactivity from two units that could be released, under nomal operating condition, from blowdown in the vapor phase to the atmosphere and in the liquid phase to the circulating water discharge tunnel has been estimated in to be $62 \mathrm{Ci}$ and $186 \mathrm{Ci}$ respectively. Bounding estimates of normal I-131 releases are:

a. Blowdown Tank Vent Pipe Release of I-131 $1.08(-1)$ to $1.78(-3) \mathrm{Ci} / \mathrm{yr}$

b. Blowdown Tank Condensate Release of I-131

$6.50(0)$ to $3.62(-1) \mathrm{Ci} / \mathrm{yr}$ 


\section{N.4 Turbine Building}

The Turbine Building houses a portion of the main steam system, turbine generators, and associated systems. The primary pathway for radioactivity to enter the Turbine Building is through leakage in the steam generator tubes, allowing primary cooling water to enter the main steam system. Thus, activity concentrations in the Turbine Building will depend on the magnitude of steam generator tube leakage. Radioactive isotopes of concern include the Iodine species in solid liquid and gaseous form, noble gases, and particulates including Cesium and Cobalt. In many cases, only information on the Iodine species was available. The system with components in the Turbine Building are:

- Main Steam Sysitem

- Turbine Generator

- Condensate and Feedwater System

- Condenser

- Lubricating 0il System

- Bearing Cooling Water System

- Auxiliary Steam System

- Circulating Water System

- Secondary Vent and Drain System.

of the above, the Lubricating 0 il System, Bearing Cooling Water System, and Circulating Water System were dismissed as systems that could contain significant quantities of radioactivity. The Secondary Vent and Drain System could potentially contain some activity, but this system is in the category of a barrier to radioactive release to the environment. The grain system collects potentially radioactive liquids and discharges them to the Liquid waste Disposal System or to the Boron Recovery System for processing. Secondary 
plant piping generally drains to the condenser. The air ejector vents through a radiation monitor, and can be diverted to Primary Containment in case of high activity level. This diversion is performed automatically. There are no gaseous filters in the Turbine Building itself, and normal gaseous emissions are released directly to the enviroment. The components of interest for the analysis were identified from the Surry heat balance diagram and from the Turbine Building architectural drawings contained in the FSAR.

\section{Release Likelihood}

No data on radioactivity levels in the components of interest in Surry was available. So the following discussion is based on coolant loops and a set of measurements $(64)$ of the activity of effluent streams in the secondary side of the Point Beach power. The activity level in the steam is about four times higher than the activity level in the feed water. The implication is that about 70 percent of the activity (principally Iodine species) is plated out in components between the steam and feedwater, since if no plate-out occurs, the activity in the feedwater is expected to be at the same level as the activity in the steam. Analysis of samples from a variety of places in the steam and feedwater systems indicated that the most likely components to have high activity levels due to plate-out were the condenser, moisture separator, and reheater.

For the Point Beach reactor, the implication from the measurements is that the combined deposition rate in the condenser, moisture separator, and reheater is $0.079 \mu \mathrm{ci} / \mathrm{hr}$. Point Beach had a combined steam generator tube leakage of $0.8 \mathrm{gal} / \mathrm{min}$ when the measurements were taken, which is fairly substantial but within Tech Spec limits (Tech Specs allow operation with up to 
$10 \mathrm{gal} / \mathrm{min}$ tube leakage). At the deposition rate derived from measurements at Point Beach, assuming that this represents the accumulated curie increase rate and the components are not cleaned, a maximum of $0.02 \mathrm{ci}$ of Iodine species would be deposited during a 30-year plant life. This represents the maximum activity that could be released from these components. This maximum activity is small compared to release magnitudes of other accident sequences analyzed, even those with fairly high probabilities.

Surry was designed with the capability of diverting the feedwater through a demineralizer and filters, whereas Point Beach does not have this option. Initially, it was speculated that these Surry components might represent repositories for significant quantities of radiation. However, if the Point Beach experience is representative, the demineralizer and filters at Surry would not be expected to contain large inventories of activity, since a large fraction of the activity is apparently plated-out in components ahead of the filters.

The conclusion is that potential radioactive sources in the Turbine Building, while possibly important for occupational exposure considerations, almost certainly do not involve dominant accident sequences that impact the risk to the public.

\section{N. 5 Fuel Building and Primary and Refueling Water Storage Tanks}

The fuel building is shown in Surry FSAR Figures 15.1-14 and 15.1-15. Sources of radioactivity have been treated in Section 3.3 and Appendices I and J. Only I-131 releases from the Spent Fuel Pool (SFP) due to loss of cooling or refueling accidents have been considered. The release of tritium has not been examined. Tritium releases in the form of tritiated water vapor are 
expected for the loss of SFP cooling accident. Nomal releases in the form of $\mathrm{T}_{2} \mathrm{O}$ and HTO occur from evaporation of SFP water but the consequences are negligible to the general public.

The tritium concentration in the SFP can be considered to be approximately equal to the concentration in the Refueling Water Storage Tank (RWST) as the RWST furnishes make-up water to SFP during normal plant operation. The origin of tritium in the RWST is from the primary reactor coolant. At the start of refueling operations the refueling reactor cavity and the refueling canal are flooded with water from the RWST. The tritium rich primary coolant mixes with the RWST water lowering the concentration of the primary coolant. However, at the end of refueling operations tritium enriched water is pumped back to the RWST. In addition, a significant amount of tritium inventory resides in the Primary Water Storage Tanks (PWST). The origin of tritium in these tanks is from the periodic bleed process of the primary coolant for deboration and purification. The RWSTs and PWSTs are located in the yard area of Surry and not in the fuel building. These tanks are mentioned in this section as they share a common denominator - significant inventories of tritium.

Tritium concentration measurements in R.E. Ginna(64) have confirned the presence of large inventories of tritium in the aforementioned tanks. A realistic estimate of tritium release based only on these measured concentrations levels cannot be performed since the concentration is a sensitive function of the precentage of ternary fission diffusion, cumulative effective full power days of operation and the tritium release management followed in a particular plant. Three tritium release management policies have been identified:

1. Zero Release Mode1, 2. Batch Release Model, 3. Continuous Release Model. 
The radiological hazard from tritium as an external radiation is of negligible consequence since tritium decays by emission of a low energy beta particle $(E \max =0.0186 \mathrm{MeV})$. Its effective half life is approximately 12 days and it does not accumulate in a particular organ, unlike iodine in thyroid glands. The health effects are primarily from inhalation and skin absorption, and ingestion of tritiated drinking water and contaminated food. A preliminary analysis based on results obtained from the Tritium Man-Rem Consequence Model (TREM) code(65) which estimated the environmental effects of normal and off-nomal releases of tritium from Controlled Themonuclear Reactor Systems show that the consequences resulting from the SFP boiling would not be very severe.

\section{N. 6 Decontamination Facility Building}

The primary purpose of the decontamination facility is to eliminate surface contamination from equipment without releasing activity to the environment. The building is made of poured concrete and concrete blocks. In Surry Power Station it is located adjacent to containment building (Unit 2) and the fuel building but separated from them to allow independent movement in the event of an earthquake. A 125 ton trolley used for transporting spent fuel cask and spent resin cask runs over the roof of the building. The roof has several hatches to provide access to the casks.

At present detailed information relating to the expected activity level of the components operational procedure and operating conditions are not available. The available information indicates the following principal components inside the decontamination building:

1. Spent Resin Catch Tank 
2. Spent Resin Dewatering Tank

3. Dewatering Tank Pump

4. Fluid Waste Treatment Tank

5. Fluid Waste Treatment Tank Pump

6. Spent Résin Casks

7. Spent Fuel Casks

Release Likelihood

All of the liquid inventory in the building is treated by the Liquid Waste Processing System before discharge to the environment. The tanks in this facility operate at low pressure, near or about atmospheric. Therefore, rupture of tanks or pump casings are highly unlikely. However, leakage from pumps and tanks are credible. The building is provided with an extensive network of sumps so any spillage would be contained within the building. The liquid in the sumps is pumped to the Liquid Waste Processing Systen for eventual release.

The building is maintained at a subatmospheric pressure. Thus any release of airborne activity is contained within the building for eventual controlled discharge. The ventilation vent is provided with a radiation monitor. Any increase in airborne activity level diverts the exhaust air through charcoal filters.

Based on the Surry FSAR Figure 9.14-1 the following accident scenario could be envisaged. The spent resin shipping container and cask sit very close (approximately 3 feet) to the Fluid Waste Treatment Tank. During transport of the spent resin cask a swing of the cask would impact the tank and possibly cause rupture. Since the roof hatch is open during this operation, 
any airborne activity contained in the tank would find a direct pathway to the atmosphere. At the present time the radioactive inventory is not known. The risk implications from this accident should be further investigated. The spent resin catch tank is also close by. However, since this tank has a concrete wall around it, a common cause failure of the spent resin catch tank under the above mentioned scenario is not considered to be credible. 


\section{ACKNOWLEDGMENTS}

The authors wish to thank Mr. J. Murphy and Dr. W. Vesely for giving valuable suggestions and comments throughout the research. They also are indebted to Mrs. S. Dyroff to aiding in the final preparation and typing of the manuscript. They also appreciate the help of $\mathrm{J}$. Zahra in the preparation and use of the codes. 


\section{REFERENCES}

1. Reactor Safety Study, "An Assessment of Accident Risks in U.S. Commercial Nuclear Power Plants, WASH-1400 (NUREG-75/014), U.S., (October, 1975).

2. CRAC Code, calculations of reactor Accident Consequences. NRC, unpublished.

3. "Instructions for preparation of data entry sheets for Licensee Event Report File," NUREG-0161, (1977).

4. System 2000, MRI Systems Corporation, Austin, Texas, (August, 1974).

5. Lindley, D.V., "Introduction to Probability \& Statistics from a Bayesian Viewpoint," CUP, 1965.

6. Spanner, J. and Gelbard, E.M., "Monte Carlo Principles and Neutron Transport Problems," Addison Wesley, 1969.

7. "Calculation of Releases of Radioactive Materials in Gaseous and Liquid Effluent from Pressurized Water Reactors," PWR-GALE Code, NUREG-0017, (Apri1, 1976).

8. RESAR-3S, Westinghouse Electric Corporation, (July, 1975), and amendments.

9. Indian Point 3 FSAR, answer to question 9.14 in Amendment 22.

10. Southern California Edison Co. letter to Division of Reactor Licensing $(A E C)$, dated February 11, 1972, (Docket 50-2060150).

11. Masche, G., "Systems Summary of a Westinghouse Electric Corporation," PWR Systems Division, (3rd. printing, August 1973).

12. Malinowski, D.D., Bell, M.J., Dunn, E.R., and Loconte, J., "Radiological Consequences of a Fuel Handling Accident," WCAP-7828, (December, 1971).

13. Brutschy, F.J., and others, "Behavior of Iodine in Reactor Water During Plant Shutdown and Startup," NEDO-10585, General Electric Co., (August 1972).

14. Neeb, K.H. and Schuster, E., "Iodine Spiking in PWR's: Origin and General Behavior," Trans. Am. Nucl. Soc. 28, 650 (June, 1978).

15. Pasedag, W.F., "Iodine Spiking in BWR and PWR Coolant Systems," Proc. ANS Topical Meeting on Thermal Reactor Safety, July 31 - August 4, 1977, Sun Valley, Idaho, pp. 3-217, CONF - 770708. 
16. P. 113 ORNL

17. P. 120 Burrell Flow Model.

18. Fontecilla, H.H. and Grimes, B.K., "Radiological Consequences of a Main Steam Line Failure with Large Steam Generator Tube Leaks, "Proceedings of Topical Meeting on Thermal Reactor Safety, CONF 770708, Sun Valley, $\underline{3}$, 3-203-215 (1977).

19. "ATWS: A Reappraisal, Part III, Frequency of Anticipated Transients," EPRI, NP-810, July 1978.

20. Thadani, A.C. et al, "An Approximate ATWS Study to Include Parameter Variations and Equipment Reliability in Probabilistic Accident Analysis." Proc. of the Topical Meeting on Probablistic Analysis of Nuclear Safety, May 8-10, 1978, Los Angeles, Vol. 2, p. II.5 to II-5-10.

21. "Westinghouse Anticipated Transients Without Trip Analysis, "Westinghouse Electric Corp., Pittsburgh, WCAP-8330, August 1974.

22. "ATWS: A Reappraisal, Part II, Evaluation of Societal Risks Due to Reactor Protection System Failure," Vol. 3, PWR Risk Analysis, EPRI, NP-265, August 1976 .

23. "Inservice Inspection of Nuclear Power Plant Components," Section XI, IWV-3510, Safety and Relief Valve Tests. ASME Boiler and Pressure Vessel Code.

24. Maekawa, M., Rasmussen, N.C., and Vesely, Jr., W.E., "A Method for Risk Analysis of Nuclear Reactor Accidents MITNE - NUREG-205 (1978).

25. Mann, N.R., Schafer, R.E., and Singpurwalla, N.D., "Methods for Statistical Analysis of Reliability and Life Data, Wiley (1974).

26. Thoman, D.R., Bain, L.J., and Antle, C.E., Technometrics, 11, 445 (1969).

27. Mann, N.R., Sheuer, E.M., and Fertig, K.W., Communication in Statute, $\underline{2}$, $383(1973)$.

28. Dickey, J.M., WBFIT, "A Code to Fit a Weibull Distribution to Data," to be published.

29. Beyer, W.H., ed., Handbook of Tables for Probability and Statistics, The Chemical Rubber Company, (1974).

30. Harter, H.L., and Moore, A.H., Technometrics I, 639 (1965).

31. Mann, N.R., Technometrics, 10, 231 (1968).

32. Sources of Radioiodine at Pressurized Water Reactors EPRI Report NP-939 (1978). 
33. Hodgman, C.D., ed. Handbook of Chemistry and Physics, The Chemical Rubber Company, (1962), p. 1708 .

34. Lutz, Jr., R.J., "Iodine Behavior Under Transient Conditions in the Pressurized Water Reactor," WCAP-8637, (November, 1975).

35. Lutz, Jr., R.J. and Chubb, W., Trans. Am. Nucl. Soc. 28, 649 (June, 1978).

36. Caruthers, G.F. and Gritz, R.W., Trans. Am. Nucl. Soc. 28, 653 (June, 1978).

37. Postma, A.K. and Tam, P.S., "Iodine Behavior in a PWR Cooling System Following a Postulated Steam Generator Tube Rupture Accident," BCT-102, Benton City Technology, Benton City, Washington, (November, 1977).

38. Eikelpasch, N., Seepolt, R. and Hock, R. Trans. Am. Nucl. Soc. 28,652 (June, 1978).

39. WCAP-8185, Westinghouse Electric Corporation.

40. Rogers, J.T., letter to Stolz, J., dated March 15, 1977 on the subject of a fuel handling accident in the Reactor Building of Crystal River Unit 3 , (Docket No. 50-302-400).

41. Schwencer, A., letter to Moore, J.B., of Southern California Edison, dated January 14, 1977, (Docket No. 50-216).

42. Proposed ANS Standard-Decay Energy Release Rates Following Shutdown of Uranium-Fueled Thermal Reactors, (October, 1971).

43. Johnson, A.B., "Behavior of Spent Nuclear Fuel in Water Pool Storage," BNWL-2256, Battel le Pacific Northwest Laboratories, (September, 1977).

44. Styrikovich, M.A., and others, "Transfer of Iodine from Aqueous Solutions to Saturated Vapor," Translated from Atomnaya Energiva, 17, (1), 45-9 (July 1964).

45. Burchsted, C.A. and Fuller, A.B., "Design, Construction, and Testing of High-Efficiency Air Filtration Systems for Nuclear Application," ORNLNSIC-65, Oak Ridge National Laboratory, (January, 1970).

46. Burchsted, C.A., Kahn, J.E. and Fuller, A.B., "Nuclear Air Cleaning Handbook," Oak Ridge National Laboratory, ERDA-76-21, (March, 1976).

47. Basin, S.L. and Burns, E.T., "Characteristics of Pipe System Failures in Light Water Reactors," EPR I NP-438, (August 1977).

48. Tong, L.S. Boiling Heat Transfer and Two Phase Flow, John Wiley and Sons, Inc., New York, 1965. 
49. Harris Fullwood, "An Analysis of the Relative Probability of Pipe Rupture at Various Locations in the Primary Coolant Loop of a Pressurized Water Reactor Including the Effect of a Periodic Inspection," SAI 001PA, (June, 1976).

50. Harris, D.0., "An Analysis of the Probability of Pipe Rupture at Various Locations in the Primary Cooling Loop of a Babcock \& Wilson 177 Fuel Assembly Pressurized Water Reactor - Including Effects of Periodic Inspection," SAI-050-77-PA, (November, 1977).

51. Bush, S.H., "Impact of ISI on Reliability of Vessels and Piping," BNWL-SA-5423.

52. Gibbons, Hackney, "Survey of Piping Failures for the Reactor Coolant Pipe Rupture Study," GEAP 4574, (March, 1974).

53. O'Neil Jordan, "Safety and Reliability Requirements for Periodic Inspection of Pressure Vessels in the Nuclear Industry," I.M.E. Proceedings, London, 1972, NRC 48/72.

54. Lindackers/Stoebe1, "Probability Analysis Applied to Light Water Reactors: Loss of Coolant Accidents," Nuclear Safety, 14-1, (JanuaryFebruary, 1973).

55. Bush, S.H., "Reliability of Piping in Light Water Reactors," IAEA-SM218/12, (October, 1977).

56. Arnet, L.M., "Optimization of Inservice Inspection of Pressure Vessels," DP1428, (August 1976).

57. Tatone, 0.S. and Pathania, R.S., "Steam Generator Tube Failures: World Experience In Water-Cooled Nuclear Power Reactors in 1976," Nuclear Safety, Vo1. 19, No. 6, November-December, 1978, p. 748-760.

58. Hare, M.G., "Steam Generator Tube Failures: World Experience in WaterCooled Nuclear Power Reactors in 1975," Nuclear Safety, Vol. 18, No. 3, May-June 1977, p. 355-364.

59. Hare, M.G., "Steam Generator Tube Failures: World Experience in WaterCooled Nuclear Power Reactors in 1974," Vol. 17, No. 2, March-April 1976 p. 231-241.

60. Regulatory Guide 1.83, Inservice Inspection of Pressurized Water Reactor Steam Generator Tubes, U.S. Nuclear Regulatory Commission, Revision 1, July 1975.

61. Regulatory Guide 1.121, Bases for Plugging Degraded PWR Steam Generator Tubes, U.S. Nuclear Regulatory Commission, August, 1976.

62. Vagins, M. "Steam Generator Tube Burst and Collapse Predictions," Presented at the Sixth Water Reactor Safety Information Meeting, Gaithersburg, Md., November 6-9, 1978. 
63. An analysis of low level solid radioactive waste from LWR's through 1975, ORP-TAD-77-2 (1977).

64. "Source Term Data for Westinghouse Pressurized Water Reactors," WCAP8253, (JuTy, 1975).

65. McKone, P.E. Environmental effects of normal and off-normal releases of tritium from TPR systems, EPRI-ER-879, 1978. 